\title{
Interactive effects of climate change and plant invasion on alpine biodiversity and ecosystem dynamics
}

\section{Justyna Giejsztowt}

M.Sc., 2013 University of Poitiers, France;

Christian-Albrechts University, Germany

B. Sc., 2010 University of Canterbury, New Zealand

A thesis submitted to Victoria University of Wellington in partial fulfilment of the requirements for the degree of

\section{Doctor of Philosophy}

School of Biological Sciences

Victoria University of Wellington

Te Herenga Waka

2019 
This thesis was conducted under the supervision of

\author{
Dr Julie R. Deslippe (primary supervisor) \\ Victoria University of Wellington \\ Wellington, New Zealand
}

\begin{abstract}
And
Dr Aimée T. Classen (secondary supervisor) University of Vermont Burlington, United States of America
\end{abstract}


"May your mountains rise into and above the clouds."

-Edward Abbey 
Drivers of global change have direct impacts on the structure of communities and functioning of ecosystems, and interactions between drivers may buffer or exacerbate these direct effects. Interactions among drivers can lead to complex non-linear outcomes for ecosystems, communities and species, but are infrequently quantified. Through a combination of experimental, observational and modelling approaches, I address critical gaps in our understanding of the interactive effects of climate change and plant invasion, using Tongariro National Park (TNP; New Zealand) as a model. TNP is an alpine ecosystem of cultural significance which hosts a unique flora with high rates of endemism. TNP is invaded by the perennial shrub Calluna vulgaris (L.) Hull. My objectives were to: 1) determine whether speciesspecific phenological shifts have the potential to alter the reproductive capacity of native plants in landscapes affected by invasion; 2 ) determine whether the effect of invasion intensity on the Species Area Relationship (SAR) of native alpine plant species is influenced by environmental stress; 3) develop a novel modelling framework that would account for density-dependent competitive interactions between native species and $C$. vulgaris and implement it to determine the combined risk of climate change and plant invasion on the distribution of native plant species; and 4) explore the possible mechanisms leading to a discrepancy in $C$. vulgaris invasion success on the North and South Islands of New Zealand. I show that speciesspecific phenological responses to climate warming increase the flowering overlap between a native and an invasive plant. I then show that competition for pollination with the invader decreases the sexual reproduction of the native in some landscapes. I therefore illustrate a previously undescribed interaction between climate warming and plant invasion where the effects of competition for pollination with an invader on the sexual reproduction of the native may be exacerbated by climate warming. Furthermore, I describe a previously unknown pattern of changing invasive plant impact on SAR along an environmental stress gradient. Namely, I demonstrate that interactions between an invasive plant and local native plant species richness become increasingly facilitative along elevational gradients and that the strength of plant interactions is dependent on invader biomass. I then show that the consequences of changing 
plant interactions at a local scale for the slope of SAR is dependent on the pervasion of the invader. Next, I demonstrate that the inclusion of invasive species density data in distribution models for a native plant leads to greater reductions in predicted native plant distribution and density under future climate change scenarios relative to models based on climate suitability alone. Finally, I find no evidence for large-scale climatic, edaphic, and vegetative limitations to invasion by $C$. vulgaris on either the North and South Islands of New Zealand. Instead, my results suggest that discrepancies in invasive spread between islands may be driven by human activity: $C$. vulgaris is associated with the same levels of human disturbance on both islands despite differences in the presence of these conditions between then islands. Altogether, these results show that interactive effects between drivers on biodiversity and ecosystem dynamics are frequently not additive or linear. Therefore, accurate predictions of global change impacts on community structure and ecosystems function require experiments and models which include of interactions among drivers such as climate change and species invasion. These results are pertinent to effective conservation management as most landscapes are concurrently affected by multiple drivers of global environmental change. 


\section{Contents}

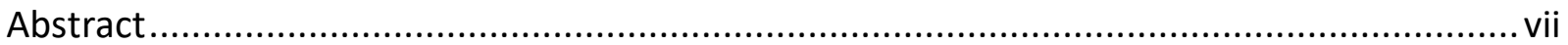

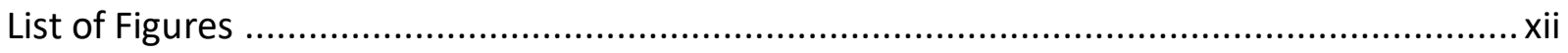

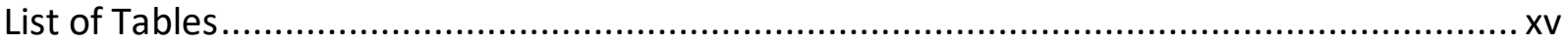

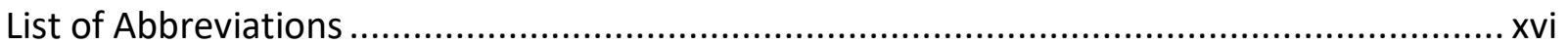

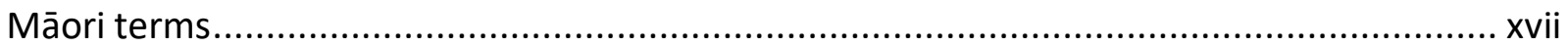

Nomenclature for focal species ....................................................................................

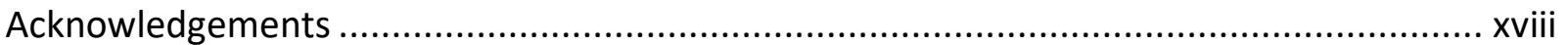

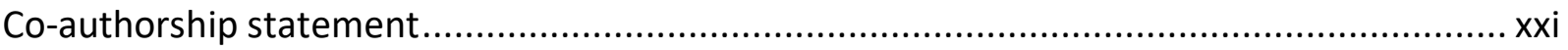

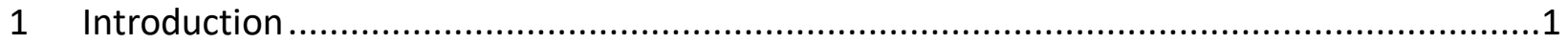

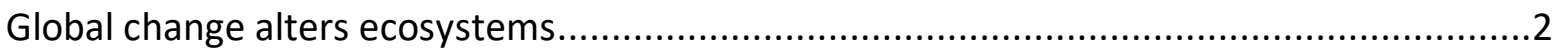

Non-linear interactions between climate warming and species invasions .......................4

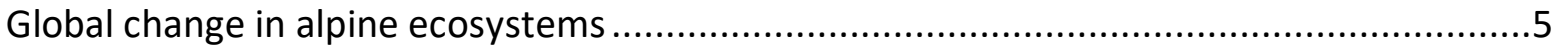

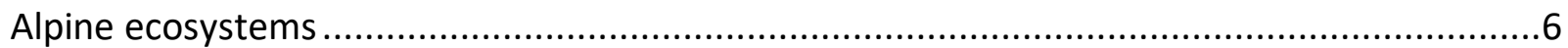

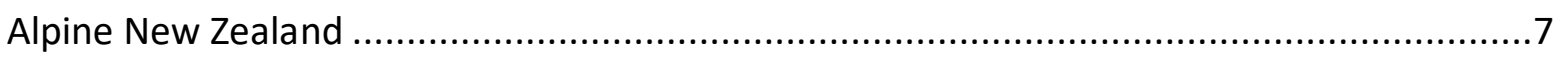

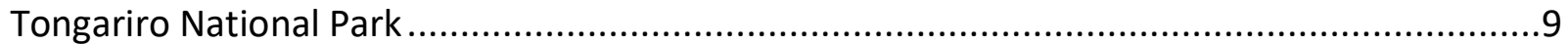

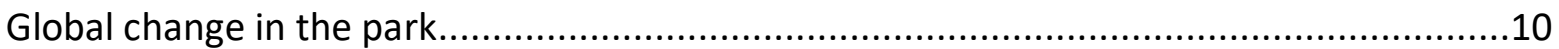

Māori as kaitiaki in the park .......................................................................

Approaches to understanding global change impacts on ecosystems ...............................14

Research objectives and outline of this thesis .........................................................20

2 Climate change and invasion synergisms may cause decline of native plant reproduction

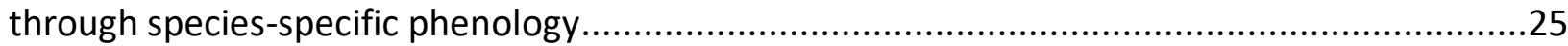

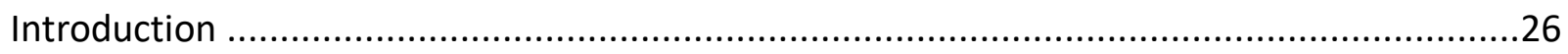

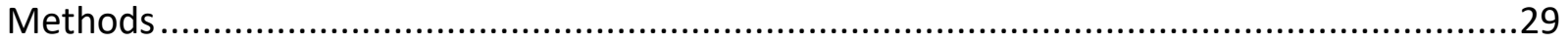

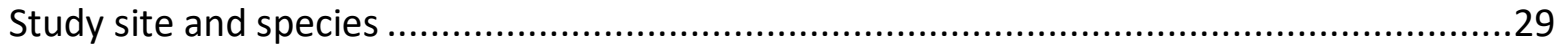

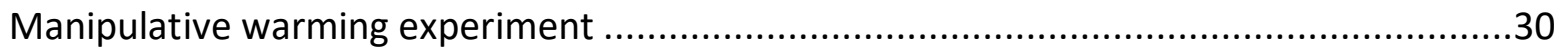

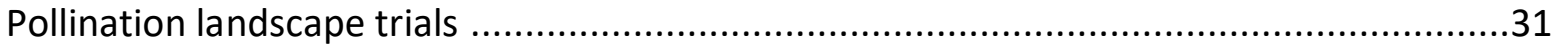

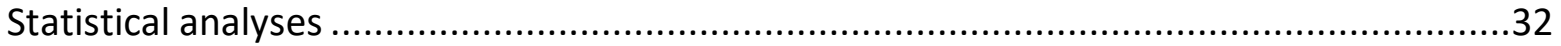

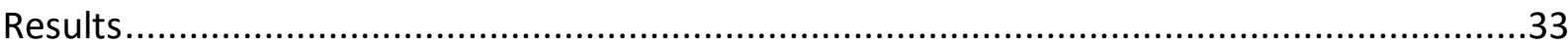

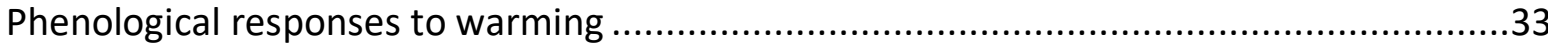

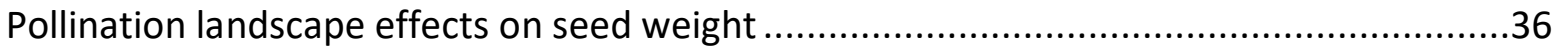


Discussion.

3 Elevational gradients alter invasion effects on species richness at multiple spatial scales ..41

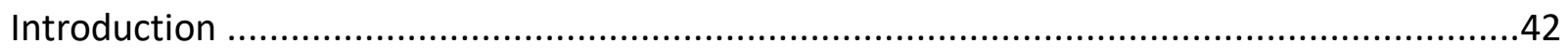

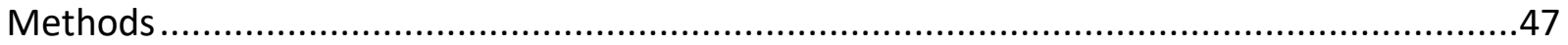

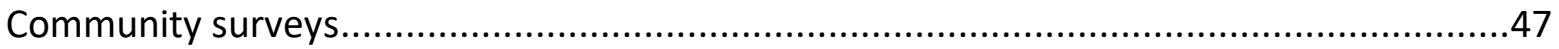

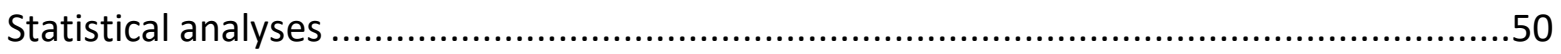

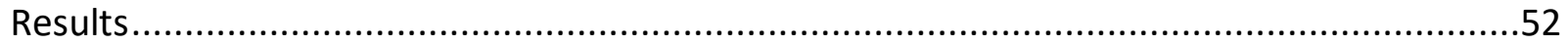

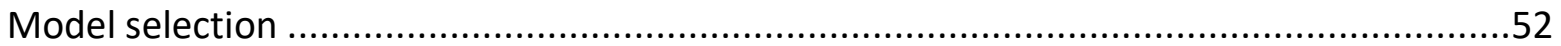

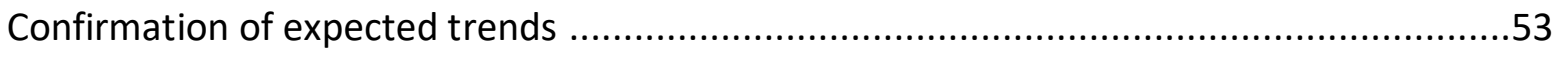

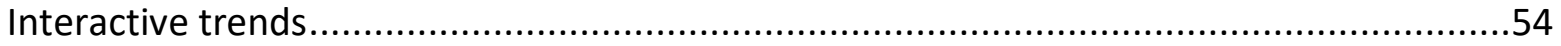

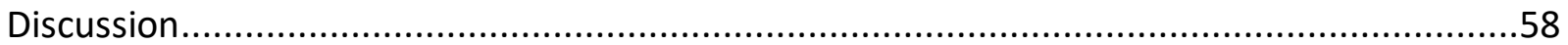

4 Density-dependent effects of an invasive species on the spatial distribution and abundance of a native species under future climate scenarios .......................................................65

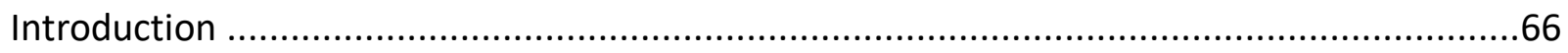

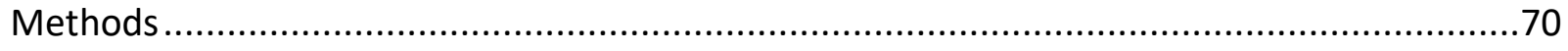

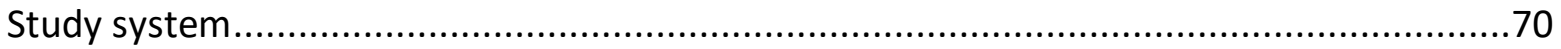

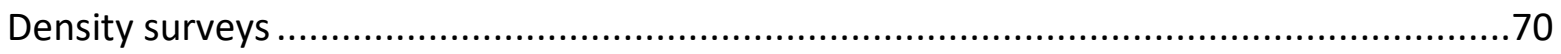

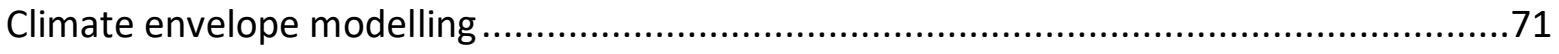

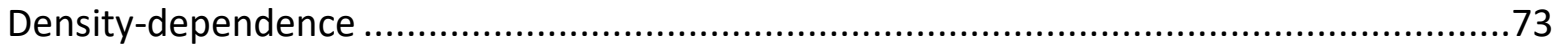

Environmental suitability-density relationship ................................................ 74

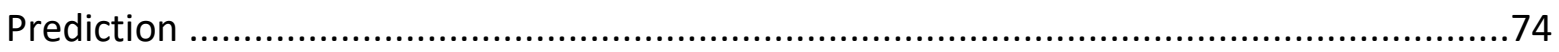

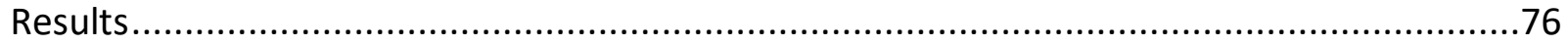

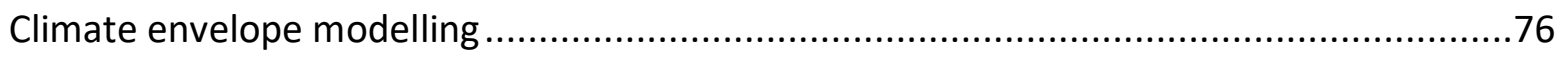

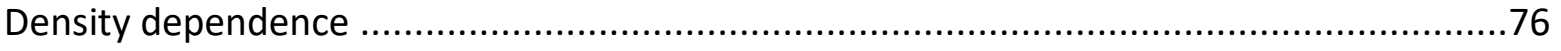

Environmental suitability-density relationship ....................................................78

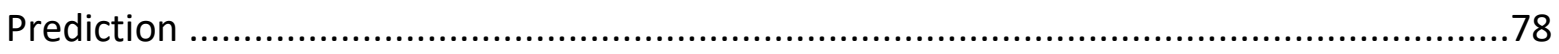

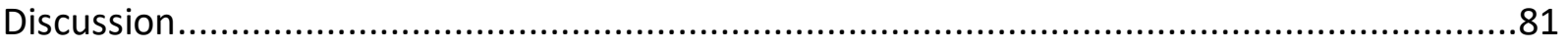

5 Inter-island discrepancies in plant invasive success are associated with human disturbance

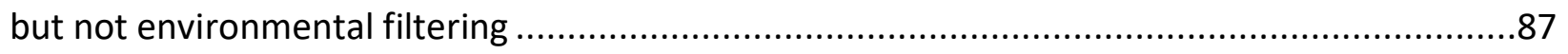

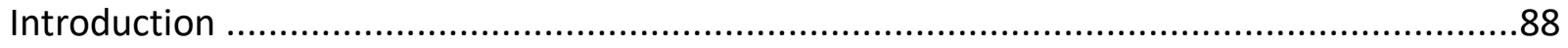

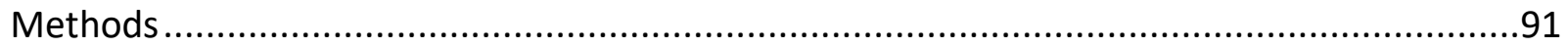




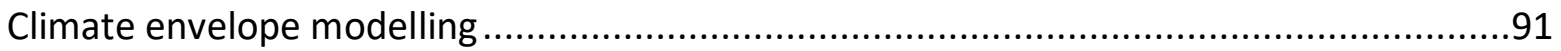

Non-climatic environmental filters .........................................................................93

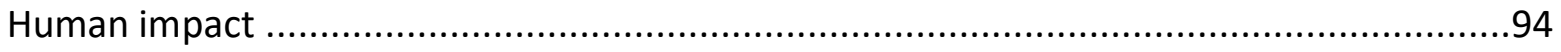

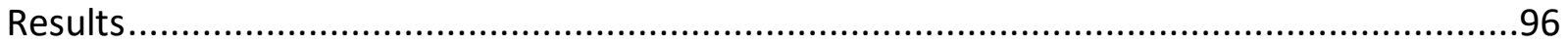

Climate envelope modelling ....................................................................................96

Non-climatic environmental filters ....................................................................99

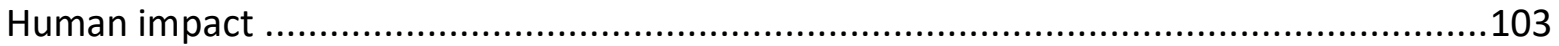

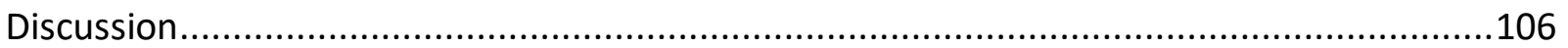

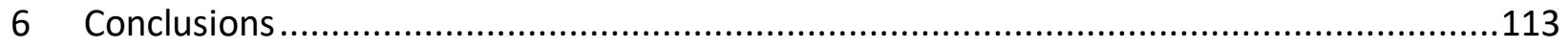

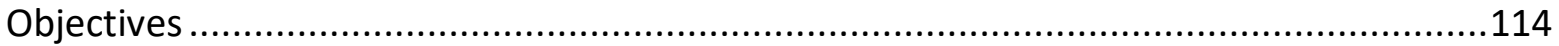

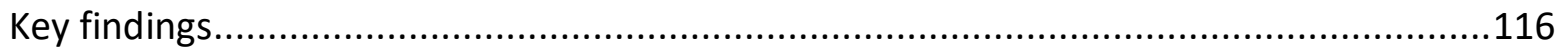

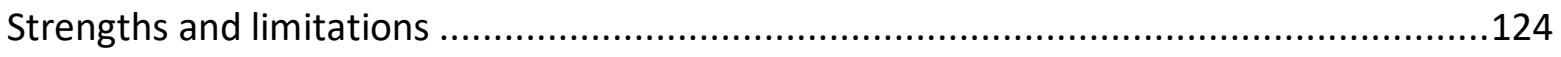

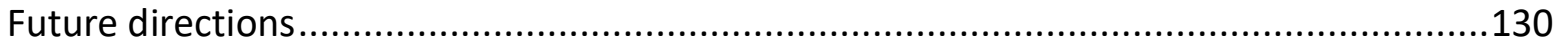

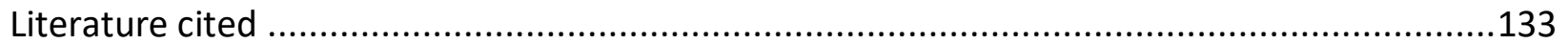

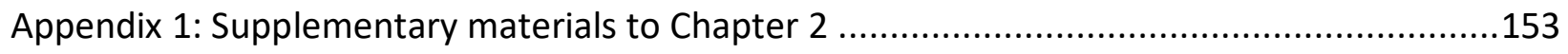

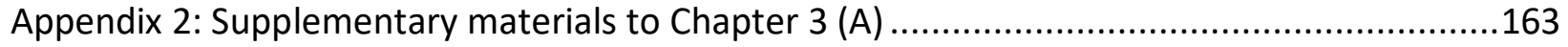

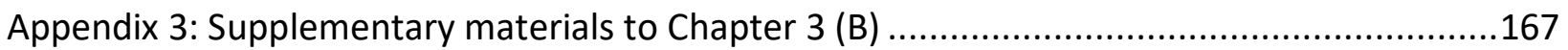

Appendix 4: Supplementary materials to Chapter 4 ...................................................171

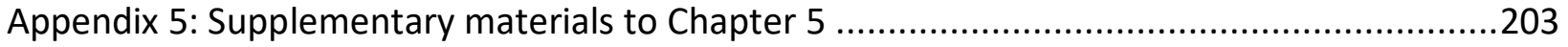




\section{List of Figures}

Figure 1.1: Theoretical framework .21

Figure 2.1: Experimental results for phenology and pollination experiments; A) floral density of Calluna vulgaris and Dracophyllum subulatum under ambient and experimentally warmed conditions; B) seed weight responses to floral density in the landscape.

Figure 3.1: Hypothesised response of plant species richness to invasion: $\mathbf{A}$ ) at the $1 \mathrm{~m}^{2}$ scale and B) on the slope of the Species Area Relationship (z). The effect of invasion should vary with environmental stress, with hypothesised effects at low environmental stress displayed in orange, and effects at high environmental stress in blue. .46

Figure 3.2: Species richness study sampling design .49

Figure 3.3: Generalized Additive Model (GAM) selection .53

Figure 3.4: Modelled native alpine plant species richness at a $1 \mathrm{~m}^{2}$ scale as a function of Calluna vulgaris biomass $\left(\mathrm{g} \mathrm{m}^{-2}\right)$

Figure 3.5: Modelled native alpine plant species richness at a $5 \mathrm{~m}^{2}$ scale as a function of Calluna vulgaris biomass $\left(\mathrm{g} \mathrm{m}^{-2}\right)$ 56

Figure 3.6: Modelled slope of the Species Area Relationship $(z)$ for native alpine plant species at a $1-5 m^{2}$ scale as a function of Calluna vulgaris canopy cover

Figure 4.1: Density dependence of Chionochloa rubra and Calluna vulagris: A) Canopy cover of C. rubra as a function of $C$. vulgaris canopy cover; B) predictions of the impact of $C$. vulgaris canopy cover on $C$. rubra canopy cover from the Boosted Regression Tree analysis.

Figure 4.2: Density of Calluna vulgaris as a function of ensemble climatic model suitability scores, presented with quantile regressions. Lines indicate the relationship as estimated by ordinary least squares regression (red line) and $97^{\text {th }}, 90^{\text {th }}, 80^{\text {th }}, 70^{\text {th }}, 60^{\text {th }}$ and $50^{\text {th }}$ quantile regressions (grey and black lines). Nagelkerke pseudo $r^{2}$ values for each relationship is provided in the figure legend.

Figure 4.3: Modelled distribution of Chionochloa rubra and its overlap with the impact niche of Calluna vulgaris in Tongariro National Park

Figure 5.1: Ensemble climatic envelope model predictions for Calluna vulgaris in New Zealand:

A) predicted climatic suitability; B) coefficient of variation across individual model predictions;

C) Multivariate Environmental Similarity Surface (MESS) comparison of training range and projection range climates methods.

Figure 5.2: Soil pH suitability for Calluna vulgaris across New Zealand .................................100

Figure 5.3: Vegetative community suitability for Calluna vulgaris across New Zealand ...........101

Figure 5.4: Mycorrhizal fungal partner presence for Calluna vulgaris across New Zealand.....102 Figure 5.5: 'Human footprint' values: A) across the North and South Islands of New Zealand; B) at Calluna vulgaris occurrences on each island.

Figure 5.6: Human disturbance intensity across New Zealand. 105

Supplementary Figure 1: Timing and location of pollination trials. 153

Supplementary Figure 2: Manipulative warming experiment treatment effect. 
Supplementary Figure 3: First flowering responses of plant species over the 2016/2017 austral summer to experimental warming.

Supplementary Figure 4: First flowering responses of plant species over the 2015/2016 austral summer to experimental warming. .156

Supplementary Figure 5: Peak floral abundance responses of plant species to experimental warming.

Supplementary Figure 6: First flowering responses by plant growth form over the 2016/2017 austral summer to experimental warming....

Supplementary Figure 7: First flowering responses by plant growth form over the 2015/2016 austral summer to experimental warming.

Supplementary Figure 8: Peak floral abundance responses to experimental warming by plant growth form.

Supplementary Figure 9: Effects of experimental treatment on seed weight of Dracophyllum subulatum.

Supplementary Figure 10: Effect of elevation $(\mathrm{m})$ on standardized Calluna vulgaris biomass (kg $\mathrm{m}^{-2}$ ) in Tongariro National Park, New Zealand.

Supplementary Figure 11: Effect of elevation $(\mathrm{m})$ on native alpine plant species richness; $\mathrm{A}$ ) hypothesized response across spatial scales; predictions fitted by GAM regressions: B) at a $1 \mathrm{~m}^{2}$ scale; C) at a $5 \mathrm{~m}^{2}$ scale; D) on the slope (z) of the Species Area Relationship.

Supplementary Figure 12: Effect of Calluna vulgaris invasion intensity on native alpine plant species richness; $\mathbf{A}$ ) hypothesized response across spatial scales; predictions fitted by GAM regressions: B) effect of biomass at a $1 \mathrm{~m}^{2}$ scale; C) effect of canopy cover at a $5 \mathrm{~m}^{2}$ scale; D) effect of canopy cover on the slope $(z)$ of the Species Area Relationship..............................166

Supplementary Figure 13: Species specific responses to Calluna vulgaris invasion ................168 Supplementary Figure 14: Boundaries of seven protected areas used to calibrate taonga tipu climate envelope models

Supplementary Figure 15: Principal Component Analysis of WorldClim "bio" predictor variables

Supplementary Figure 16: Performance of quantile regression in predictive Calluna vulgaris percentage cover as a function of environmental suitability 181

Supplementary Figure 17: Climatic suitability of Tongariro National Park (TNP) for Calluna vulgaris under the current climate, and two climate scenarios for the year 2070. .183

Supplementary Figure 18: The percentage cover of Anisotome aromatica decreases with increasing percentage cover of Calluna vulgaris....

Supplementary Figure 19: Climatic suitability of Tongariro National Park (TNP) for Anisotome aromatica and the space within which it interacts with Calluna vulgaris under the current climate, and two climate scenarios for the year 2070. 186

Supplementary Figure 20: The percentage cover of Chionochloa rubra decreases with increasing percentage cover of Calluna vulgaris 188 
Supplementary Figure 21: Climatic suitability of Tongariro National Park (TNP) for Chionochloa rubra and the space within which it interacts with Calluna vulgaris under the current climate, and two climate scenarios for the year 2070 189

Supplementary Figure 22: The percentage cover of Coprosma perpusilla decreases with increasing percentage cover of Calluna vulgaris

Supplementary Figure 23: Climatic suitability of Tongariro National Park (TNP) for Coprosma perpusilla and the space within which it interacts with Calluna vulgaris under the current climate, and two climate scenarios for the year 2070.

Supplementary Figure 24: The percentage cover of Dracophyllum recurvum is unaffected by the percentage cover of Calluna vulgaris.

Supplementary Figure 25: Climatic suitability of Tongariro National Park (TNP) for

Dracophyllum recurvum and the space within which it interacts with Calluna vulgaris under the current climate, and two climate scenarios for the year 2070 195

Supplementary Figure 26: The percentage cover of Gentianella bellidifolia is unaffected by the percentage cover of Calluna vulgaris.

Supplementary Figure 27: Climatic suitability of Tongariro National Park (TNP) for Gentianella bellidifolia and the space within which it interacts with Calluna vulgaris under the current climate, and two climate scenarios for the year 2070. .198

Supplementary Figure 28: Modelled suitable habitat area for five taonga tipu 199 


\section{List of Tables}

Table 4.1: Performance of six quantile regressions in predicting the environmental suitability-

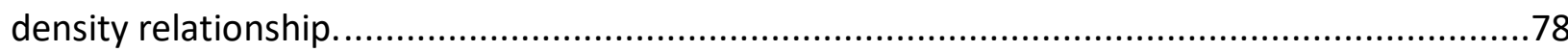

Table 5.1: Performance of Calluna vulgaris climatic envelope models...................................97

Supplementary Table 1: Summary of site-specific linear models predicting Calluna vulgaris biomass $\left(\mathrm{kg} \mathrm{m}^{-2}\right)$ based on canopy cover.

Supplementary Table 2: The performance of various forms of Generalized Additive Model (GAM) used to predict native alpine plant species richness at a $1 \mathrm{~m}^{2}$ and $5 \mathrm{~m}^{2}$ scales, and the slope $(z)$ of the Species Area Relationship

Supplementary Table 3: Rationale for selecting five taonga tipu for modelling in this study...173 Supplementary Table 4: Model performance for each species.

Supplementary Table 5: Environmental predictors used to calibrate the climatic model for Calluna vulgaris

Supplementary Table 6: Environmental predictors used to calibrate the climatic model for Anisotome aromatica.

Supplementary Table 7: Environmental predictors used to calibrate the climatic model for Chionochloa rubra

Supplementary Table 8: Environmental predictors used to calibrate the climatic model for Coprosma perpusilla.....

Supplementary Table 9: Environmental predictors used to calibrate the climatic model for Dracophyllum recurvum

Supplementary Table 10: Environmental predictors used to calibrate the climatic model for Gentainella bellidifolia

Supplementary Table 11: Area of Tongariro National Park ( $796 \mathrm{~km}^{2}$ total) that is predicted to be currently habitable by five alpine taonga tipu species and that will be habitable under climatic predictions for 2070 200 
Abbreviation Definition

ANN Artificial Neural Network

AUC Area Under the Curve

BIOCLIM Bioclimatic variables, as provided by worldclim.org

$B R T$ Boosted Regression Tree

$B S B I$ Botanical Society of Britain and Ireland

c The intercept of the Species Area Relationship

CIMP5 Coupled Model Intercomparison Project Phase 5

CTA Classification Tree Analysis

GAM Generalized Additive Model

GBIF Global Biodiversity Information Facility

GCM Global Circulation Model

GEC Global Environmental Change

GLM Generalized Linear Model

HII Human Impact Index

IPCC Intergovernmental Panel on Climate Change

JSDM Joint Species Distribution Model

LRIS Land Resource Information Systems portal

MARS Multiple Adaptive Regression Spline

MESS Multivariate Environmental Similarity Surface

NZLRI New Zealand Land Resource Inventory

$R C P$ Representative Concentration Pathway

$R F$ Random Forest

ROC Receiver Operating Characteristic

$S A R$ Species Area Relationship

SDM Species Distribution Model

SRE Surface Range Envelope

TNP Tongariro National Park

TSS True Skill statistic

UNESCO United Nations Educational, Scientific and Cultural Organization

$z \quad$ The slope of the Species Area Relationship 


\begin{tabular}{|c|c|}
\hline Term & Translation \\
\hline Aotearoa & New Zealand- "land of the long white cloud" \\
\hline$i w i$ & tribe \\
\hline kaitiaki & guardian \\
\hline kaitiakitanga & guardianship \\
\hline mātauranga Māori & traditional Māori knowledge or wisdom \\
\hline mōhiotanga & knowledge \\
\hline pākahe & elder \\
\hline taiao & The environment; natural world \\
\hline tangata whenua & the people of the land; indigenous people \\
\hline taonga & treasured \\
\hline taonga tipu & treasured plant species \\
\hline te reo Māori & Māori language \\
\hline whakawhetai ki a koe & thank you \\
\hline
\end{tabular}

Nomenclature for focal species

Species Authority

Anisotome aromatica Hook. f.

Calluna vulgaris (L.) Hull

Chionochloa rubra Zotov

Coprosma cheesemanii W. R. B. Oliv.

Dracophyllum recurvum Hook. f.

Dracophyllum subulatum Hook. f.

Gentianella bellidifolia (Hook. f.) Holub 


\section{Acknowledgements}

I am indebted to many people who have contributed to the success of this project. The past three years have been wild, and I have utmost gratitude to Dr. Julie Deslippe who rode all the waves with me. Thank you for giving me creative freedom with this project- and your patience with my million-and-one rabbit holes. For rising to the challenge of my obscure statistical hangups. For your faith in me, during all the months when I was up a mountain doing who-knowswhat. For the support, particularly in these last couple of months, and reminding me to celebrate my successes. Thank you also to Dr. Aimée Classen, your positivity is unreal, and your feedback on my writing has been so critical. Thank you for always encouraging me to aim high. For hosting me in Copenhagen and Burlington and providing me with a lot of fantastic opportunities to meet interesting people! Also thank you to Dr. Nathan Sanders, your input, particularly at the beginning of my PhD, was pivotal to the development of this project.

My PhD involved a phenomenal 13 months of field work. I have the absolute privilege of calling Tūkino my second home. Whakawhetai ki a koe, koro Ruapehu, for having me. My field assistants, each of whom spent considerable time with me in isolation, maintaining focus over long hours and putting up with my (sometimes terrible) cooking, high expectations and walking pace. I thank Garth Fabbro, Antonia Hoeta, Sunny Barham, Daniel Davis and Greg Newman. Many others took time out of their personal lives to do either do some form of terribly monotonous work, or to carry around big rocks, sometimes in difficult conditions- Julie Deslippe, Natascha Lewe, Maedeh Jafari Rad, Bobby Lust, Wim Coosemans and Nicola Durrant. I am grateful to the Tūkino Alpine Sports Club who made me feel like I was part of a community. Particularly Don French and Mark Hensen who supported my work and helped me with all those handyman tasks which I was so ill prepared for!

I have had to learn all sorts of new skills, and I thank all the people who have taken the time to teach me. The process of learning to model in relative academic isolation was challenging. I would like to thank André Bellvé and Charlie Clark whose statistical comments were critical. Varun Venkatesh, for always taking the time to talk me through inane problems. Thank you to the other two members of my supervisory committee- Dr. Stephen Hartley and Dr. Kevin Gould for giving me important feedback at critical stages of this project. Thank you, 
Matthew Biddick for vital assistance with manuscript preparation and the manuscript submission process. For feedback on chapter drafts: Dea Majstorović, Elizabeth Mittell, Emma Garnett and Dr. Michael Jackson. Finally, for technical assistance, Neville Higgison; Mary Murray, Mark Stephens and Stephen Meyers for admin support.

For funding, I would like to thank the University of Victoria Doctoral Scholarship who paid my living costs and student fees. The Centre for Biodiversity and Restoration Ecology (CBRE) travel grant, and the Faculty Strategic Research Grant which allowed me to travel to Louisiana in the United States to present my work at the Ecological Society of America conference. I am also grateful to the CBRE for granting me a student award, which contributed towards fieldwork costs. I would like to thank the Ross Beaver Mycological Trust Fund, for contributions to field work costs. We received major financial contributions from The Ministry of Business Innovation and Employment's Te Pūnaha Hihiko Fund RTVU1511-CR-1 which payed for substantial fieldwork costs.

I thank Ngāti Rangi for ongoing collaboration on our projects in Tongariro National Park. I thank Ngāti Tūwharetoa for access to land and in particular Bubs Smith for his assistance and consultation on site access. Surveys were carried out in Tongariro National Park under Department of Conservation permit \#40300- RES and we thank Hollei Gabrielsen and David Milner for supporting this application. I am grateful to the Botanical Society of Britain and Ireland who provided data from the United Kingdom.

On a personal note, this PhD has been full of ups and downs. I couldn't have done it without the people who always came out for me in support when I needed them most. My parents, Dorota and Witold, for always being there with support and wisdom. Elizabeth Mittell, for always being there to pick up the phone, no matter the time of day. You are a friend indeed! Sam Read and Rohan Batt. Sam, I couldn't have got through this without your unending well of empathy. Olek Giejsztowt and Dea Majstorović- for always approaching me with open hearts. Julian, for re-roofing my house without even asking. The people who have made Wellington feel like home - Diana Weinfurtová, Oliver Cain, Rossella Angeletti, Matthew Biddick, Michael 
Jackson, Dr. Nyree Fea. All the other students with whom I shared the trials and tribulations of postgraduate life. I particularly want to thank Wim Coosemans, for everything. 


\section{Co-authorship statement}

Chapters 1 and 6 of this thesis are written in the first person singular tense, reflecting my leading role as the author of this body of work. However, Chapters 2-4 are the culmination of both my own contributions and the work of other co-authors and were written for publication as a multi-authored manuscript. Therefore, these chapters are written in the first-person plural tense. The text below outlines these contributions, as well as highlighting the how these data and results have been presented by myself and by others prior to the submission of this thesis.

Chapter 2 was co-authored by Dr. Julie Deslippe and Dr. Aimée Classen, who assisted with hypothesis development methodology design. To investigate phenological responses to manipulative warming, a pre-existing experimental setup which was designed by Aimée and Dr. Nathan Sanders as part of a collaborative project Warming And species Removal in Mountains (WARM). The experimental design was implemented by Julie. I collected all field data and maintained treatments with the assistance of Garth Fabbro, Sunny Barham and Antonia Hoeta. I designed the pollination study and collected all field data. I conducted statistical analyses in consultation with Julie and Aimée. All authors contributed to the interpretation of the data. I led the writing of the manuscript with critical contributions on content from Julie and Aimée. The manuscript draft was edited by Matthew Biddick. A version of this work has been submitted for publication and is under review. A portion of this dataset was presented at an invited seminar in Copenhagen.

Giejsztowt, J., Classen, A. and J. Deslippe. 2019. Climate change and invasion synergisms may cause decline of native plant reproduction through species-specific phenology. Manuscript has been submitted to Ecology and is currently under review.

Giejsztowt J., Classen, AT. and Deslippe JR. Interactive effects of climate change and species composition on alpine biodiversity and ecosystem dynamics. Invited Seminar. Centre for Macroecology, Evolution and Climate Seminar Series, University of Copenhagen, Denmark. $14^{\text {th }}$ September 2016

Additionally, a version of the phenology data in this chapter has been presented for two years in an undergraduate course by Julie. 
Giejsztowt, J., Classen AT. and Deslippe, J. 2016 and 2017. Evidence for climate change responses in alpine and arctic ecosystems. Lecture. Victoria University of Wellington, BIOL325. Wellington, New Zealand.

Chapter 3 was co-authored by Nathan Sanders, Aimée Classen and Julie Deslippe. Nathan and I conceived the ideas and designed methodology. I selected study sites and collected data; Sunny Barham, Antonia Hoeta and Daniel Davis assisted with field data collection. I analysed the data with assistance from Nathan and Julie. All authors contributed to the interpretation of the data. I led the writing of the manuscript with critical inputs from Julie. Elizabeth Mittell edited an early version of the manuscript. I presented a portion of this analysis (Appendix 3) at The Society for Conservation Biology- Oceania Section conference in Wellington.

Giejsztowt J., Classen, AT. \& Deslippe JR. Climate change and weed invasion synergistically affect species richness in an alpine plant community. Society for Conservation Biology-5th Oceania Congress. Wellington, New Zealand, 2-6 July 2018.

Chapter 4 was co-authored by Aimée Classen and Julie Deslippe. I conceived the ideas and designed methodology with support and feedback from Julie. I collected the field data with assistance from Sunny Barham, Antonia Hoeta and Daniel Davis. I analysed the data. All authors contributed to the interpretation of the data. I led the writing of the manuscript. All authors contributed critically to the drafts. Elizabeth Mittell edited the manuscript. I presented this work at the Ecological Society of America and the New Zealand Ecological Society conferences. I also presented this work as a lecture for the undergraduate course BIOL325 at Victoria University. I presented an early version of this work at a postgraduate symposium.

Giejsztowt J., Classen, AT. \& Deslippe JR. Climate change, plant invasion and their interactive effects on species distributions. New Zealand Ecological Society, Wellington, New Zealand, 22 November 2018.

Giejsztowt J. Species Distribution Modelling and interacting drivers of Global Environmental Change. Guest Lecture. Victoria University of Wellington, BIOL325. Wellington, New Zealand, 12 October 2018. 
Giejsztowt J., Classen, AT. \& Deslippe JR. Climate change and invasive weeds act synergistically to alter the distribution of culturally important alpine plant species in New Zealand. Ecological Society of America Annual Meeting, New Orleans, United States of America, 8 August 2018.

Biodiversity and Restoration Ecology Postgraduate Symposium, Victoria University of Wellington. 27 $7^{\text {th }}$ May 2017.

Chapter 5 was co-authored by Aimée Classen and Julie Deslippe. I conceived the ideas and designed methodology with substantial input from both Aimée and Julie. I analysed the data. All authors contributed to the interpretation of the data. I led the writing of the manuscript with significant inputs from both Julie and Aimee. Emma Garnett edited the manuscript. I presented this work at the New Zealand Plant Conservation Network conference.

Giejsztowt J., Classen, AT. \& Deslippe JR. Modelling the risk of invasive expansion risk for Calluna vulgaris on the South Island of New Zealand. New Zealand Plant Conservation Network Biennial Conference. Hokitika, New Zealand, 16th November 2017. 
1 Introduction

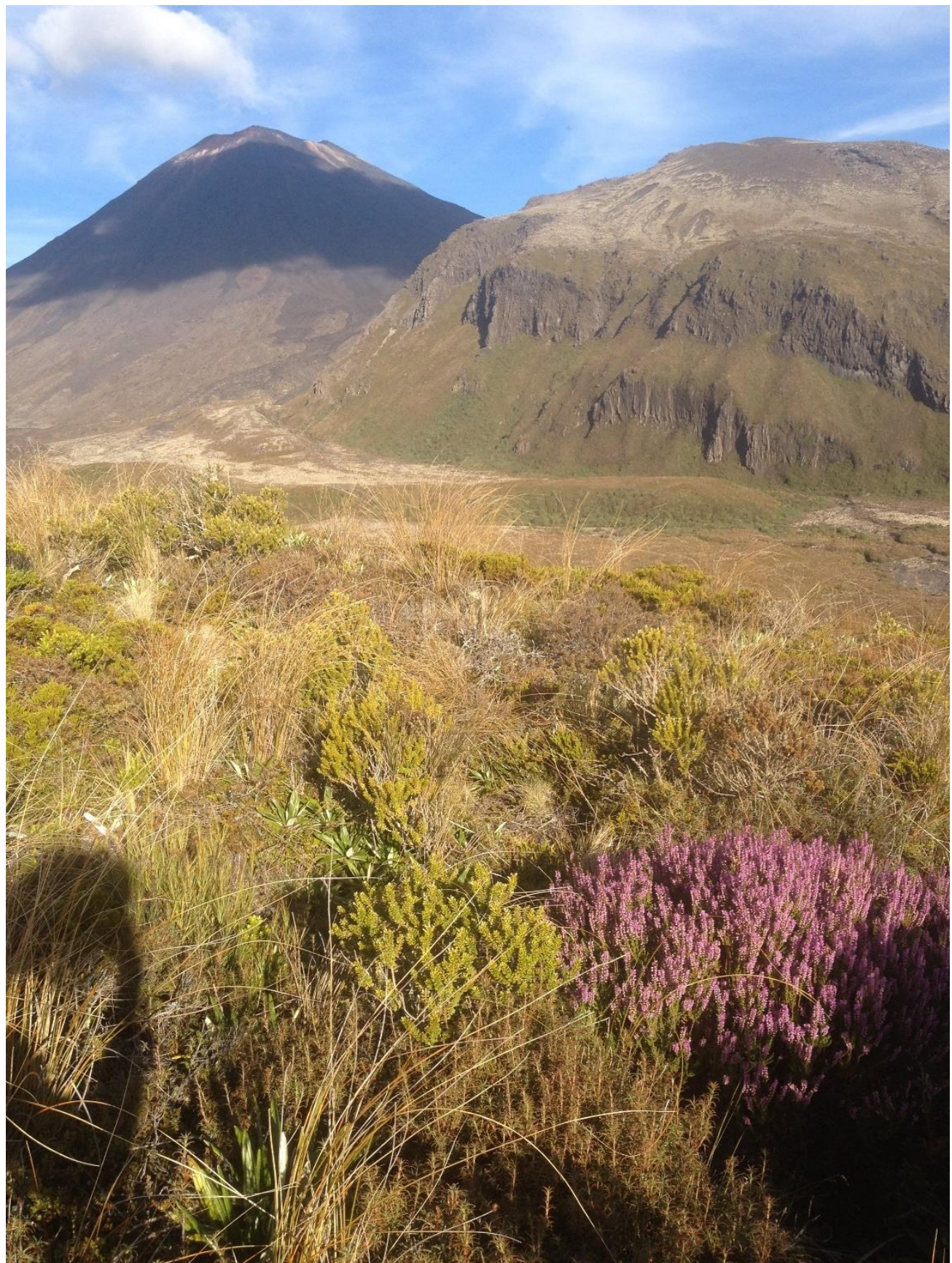

Mt. Ngauruhoe and Pukekaikiore tower over the Mangatepopo valley in Tongariro National Park. In the foreground, Calluna vulgaris (pink) encroaches into a diverse native community.

Giejsztowt, 2017 


\section{Global change alters ecosystems}

Anthropogenic alterations to the environment are causing the sixth major extinction of life on Earth with global extinction rates 100 - 1000 times higher than pre-Anthropocene estimates (Chapin et al. 2000). Biodiversity losses are a result of numerous drivers of global environmental change (hereafter: "global change"), such as land use change, growing atmospheric concentrations of greenhouse gasses, increased global mobility of organisms and increased concentrations of bioavailable nitrogen (Vitousek et al. 1997, Chapin et al. 2000, Mack et al. 2000, Vila et al. 2011). In addition to loss of biodiversity, the ecological impacts of these environmental stressors include shifts in ecosystem structure and functioning. Consequently, services provided by ecosystems that are important to the wellbeing of people are also threatened by global change (Costanza et al. 1997). Ecosystem responses to global change are inconsistent and non-linear (Chapin et al. 2000, Vila et al. 2011). With interactions possible between all drivers of global change, this dissertation focusses on contributing to scientific knowledge on the interaction between climate change and plant invasion.

Human activities, since the beginning of the industrial revolution, have dramatically increased atmospheric concentrations of greenhouse gasses such as carbon dioxide and methane which are linked with changes in global climatic patterns (Vitousek et al. 1997, Chapin et al. 2000, Isaac and Williams 2013). In terrestrial ecosystems, climate change is likely to have significant impacts on temperature, precipitation patterns and snow and ice cover, as well as the severity and frequency of extreme weather events such as EI Niño (IPCC 2014). The Intergovernmental Panel on Climate Change (IPCC) predicts with high certainty that global mean temperatures will rise by at least $1.5^{\circ} \mathrm{C}$ by 2100 relative to pre-industrial levels (IPCC 2014). The Earth's climate has already warmed by approximately $0.6^{\circ} \mathrm{C}$ over the last century (Walther et al. 2002). Such rates of change far exceed those recorded over the last 10,000 years (Isaac and Williams 2013). These trends are important in regards to ecological systems as climatic variables are crucial determinants of species diversity and distributions, and affect the provision of ecosystem services (Flanagan et al. 2013). There is substantial evidence that ecological systems are responding to recent climatic change (Walther et al. 2002, Parmesan and Yohe 2003, Parmesan 2006). Recorded changes and predicted future changes include 
distributional shifts (Brusca et al. 2013), shrinking habitats in alpine areas (Halloy and Mark 2003), altered biogeochemical cycling (Chapin et al. 2000) altered phenology (Arroyo et al. 2013) and shifts in community composition and species interactions (Walther et al. 2002). These responses result in exacerbated extinction risk for non-random species (Isaac and Williams 2013). While some effects of climate change on the structure and function of plant communities are recorded consistently across ecosystems, the consequences of these changes for biodiversity and key ecosystem services are uncertain in many cases (Walther et al. 2002, Lal 2004, Schweiger et al. 2012, Coleman 2013, Mohan et al. 2014). This is partially because climate change affects regions asymmetrically and because species within systems respond idiosyncratically (Walther et al. 2002).

Invasive plant species are common in many ecosystems (Mack et al. 2000) and the rates of species invasions are accelerating globally (Horvitz et al. 2017). Invasive plants are a subset of the non-native species in a recipient environment - those which spread and become ecologically or economically detrimental (Simberloff 2009, Ehrenfeld 2010, Pairon et al. 2010). The likelihood of plant invasion is explained, in part, by the characteristics of the species and of the recipient ecosystem (Theoharides and Dukes 2007). There are four stages to the invasion process - transport, colonisation, establishment and spread; different plant traits of the nonnative species and filters in the recipient ecosystem contribute to the success of the invasive species at each stage (Theoharides and Dukes 2007). Plant invasions typically affect ecological systems by decreasing the abundance and diversity of native plant species, increasing primary production and altering several other ecosystem processes (Vila et al. 2011). However, the ecosystem impacts of invasive species are notoriously inconsistent in magnitude and direction among ecosystems and can be dependent on the functional identity of the invasive species (Theoharides and Dukes 2007, Vila et al. 2011). Invasive species alter ecosystems by diverting energy or biochemical flows, altering fundamental ecosystem qualities, competing directly with other plant species for resources, or by disrupting existing mutualisms, amongst other mechanisms (Chapin et al. 2000, Chittka and Schurkens 2001, Brown et al. 2002). Further, impacts of invasive species may vary within ecosystems depending on the scale of investigation (Chase and Knight 2013, Powell et al. 2013, Rejmanek and Stohlgren 2015, Chase et al. 2018). 


\section{Non-linear interactions between climate warming and species invasions}

Few, if any, ecosystems are subject to pressure from a singular driver of global change acting in isolation. On the contrary, dramatic transformations of ecosystems are often the result of multiple drivers of global change acting synergistically (Walther et al. 2002, Theoharides and Dukes 2007, Tylianakis et al. 2008, Hoover et al. 2012, Root et al. 2013). While climate warming is likely to directly alter ecosystem structure and function, indirect effects of warming that are mediated through species interactions (González-Varo et al. 2013, Classen et al. 2015, Farrer et al. 2015) or involve interactions with other drivers of global change (Brook et al. 2008, Root et al. 2013) are predicted to be the greatest threat to biodiversity and ecosystem functioning globally. For example, climate change may facilitate species invasions by reducing the resilience of existing plant communities (MacDougall and Turkington 2005, Sheppard et al. 2016), facilitate species establishment (Walther et al. 2002) or alter competitive conditions between native and existing invasive species (Schweiger et al. 2010, Sheppard et al. 2016). Conversely, plant invasions may buffer ecosystems from climate change driven extinctions, for example by providing additional connectivity in mutualistic networks (Schweiger et al. 2010). None-the-less, species extinctions attributed to global warming are often driven by synergisms with invasion, and commonly occur through exacerbated competitive pressure (Brook et al. 2008). 


\section{Global change in alpine ecosystems}

Alpine ecosystems are vulnerable to climate change (Körner et al. 2011, Seddon et al. 2016) because these systems are predicted to be subject to particularly high rates of warming (Callaway et al. 2002). Climate change alters temperature and precipitation regimes, which directly drive alpine biodiversity patterns (Engler et al. 2011). There is ample evidence of global change effects in alpine ecosystems. Rising tree and shrub lines are a prominent response to rapid climatic change in these ecosystems (Danby and Hik 2007, Seddon et al. 2016). The distribution of alpine species are shifting upslope globally at a rate of $6.1 \mathrm{~m}$ decade ${ }^{-1}$ (Parmesan and Yohe 2003). Alpine ecosystems might be particularly affected by climatic change because alpine habitats are fragmented, and as the climate warms, high alpine species tracking colder climates in a warming world may lack an "escape route" (Halloy and Mark 2003). However, the role of climate change in alpine ecosystems is debated, as high topographic variability may buffer these ecosystems from climate change driven effects; this complexity makes predictions for alpine ecosystem responses to climate change particularly difficult (Ashcroft et al. 2012, Körner 2013, Lord et al. 2018). For example, alongside climate warming, climate change models indicate that windspeed and direction are likely to change in New Zealand (Ministry for the Environment 2018). Climate change driven alterations to wind speed may interact with complex topography to alter snow cover patterns, a crucial determinant of local abiotic conditions and therefore biodiversity patterns (Inouye et al. 2000, Winstral et al. 2002, Lord et al. 2018). Further, climate warming may occur asymmetrically across the year, and species may respond idiosyncratically to alterations in summer and winter temperatures (Bokhorst et al. 2012). Alpine plant species typically exhibit extreme phenotypic plasticity that may preclude high species extinction rates (Lord et al. 2018). However, even plastic responses may be insufficient to guarantee a species' survival, if, for example, species' phenology responds primarily to inappropriate cues (Inouye 2008).

Species invasions are historically unimportant in alpine ecosystems (Alexander et al. 2016), however, rates of invasion are increasing due to rising propagule pressure driven by road development and other human activities (Dainese et al. 2017). Additionally, climate warming is increasing colonisation by species that were historically excluded from alpine areas by harsh 
abiotic conditions (Gottfried et al. 2012). In addition to invasions of exotic plants, colonisation of alpine ecosystems by lowland species is facilitated by climatic change (Vittoz et al. 2013, Alexander et al. 2016). Colonisation of both exotic and lowland species leads to an increase in species richness in high alpine zones (Vittoz et al. 2013) as well as species homogenisation among alpine areas (Jurasinski and Kreyling 2007). Understanding interactions among global change drivers and indirect effects mediated by species interactions is crucial if we are to predict the diversity and function of alpine ecosystems in the future (Lord et al. 2018).

\section{Alpine ecosystems}

The defining feature of mountain ecosystems is their geomorphic steepness (Körner et al. 2011). Alpine areas are those portions of mountain ecosystems that lie above the climatic treeline in non-arctic areas (Bliss 1962, Mark 2012). Vascular plants typically occur to about 1000-1500m above the treeline (Körner 2013) and plant communities are structured into bioclimatic bands, driven by temperature lapse rates along elevational gradients (Körner et al. 2011). Consequently, alpine biomes host high environmental heterogeneity over small geographic areas (Körner et al. 2011). Microhabitats are an important feature of all alpine ecosystems because relief, exposure and slope interact with wind and solar radiation to create differences in soil moisture, soil quality and temperature over fine scales (Körner 2013). For this reason, despite similarities with arctic tundra, alpine flora have significantly higher species richness (Körner 2013). Notably, alpine ecosystems occupy only 5\% of the world's land area and harbour approximately 10,000 plant species (Cavieres et al. 2014). Harsh environmental conditions, repeated historical glacial disturbance and geomorphological constraints exclude many species that are maladapted to alpine conditions (Cavieres et al. 2014) resulting in a flora which is often highly endemic to alpine zones. Abiotic conditions vary among alpine ecosystems, but typically include extreme cold, high temperature variability, intense solar radiation and soil instability driven by erosion and frost heave (Bliss 1962). Therefore, alpine ecosystems are typified by specialized, hardy, slow growing, low stature vegetation (Körner 2013). At high elevation, abiotic restrictions become an increasingly important driver of species occurrence patterns. Consequently, the Stress Gradient Hypothesis (SGH) postulates that the role of plant-plant facilitation becomes increasingly significant with elevation (Cavieres et al. 
2014). In fact, high elevation nurse plants, such as cushion growth forms, may be important for global scale species richness patterns (Callaway et al. 2002, Cavieres et al. 2014).

Alpine ecosystems provide critical services to humans, particularly through water provision and regulation (Beniston 2003) and diverse alpine plant communities contribute to the stability and quality of this resource through soil stabilisation (Pohl et al. 2009). Approximately $40 \%$ of the human population relies on water from mountain watersheds (Beniston 2003). Additional ecosystem services include recreational activities, energy, mineral resources, grazing land and forestry (Beniston 2003, Körner 2013). Despite these services, alpine zones are not equally studied globally; studies of the alpine flora are disproportionately based in Europe (Abbott 2008).

\section{Alpine New Zealand}

There are approximately 700 vascular taxa above the treeline in Aotearoa, New Zealand (Mark 2012). Many are endemic (93\%), with woody plants having the highest rates of endemism (McGlone et al. 2001). While some floral taxa in New Zealand are Gondwanan relicts, most taxa originate from long distance dispersal events, notably from the Antarctic continent (Mark 2012) or more recently, during the Late Miocene and early Pleistocene period, from Australia (McGlone et al. 2001). Taxa originating from Australia are often derived from tropical and subtropical ancestral lineages which have adapted to a cooling climate (McGlone et al. 2001). Some genera also have South American or Northern Hemisphere origins (Mark 2012). The endemic alpine flora of New Zealand is young, having evolved to alpine conditions after the rapid uplift of the Southern Alps 4 Mya (McGlone et al. 2001, Winkworth et al. 2005, Mark 2012). During the late Oligocene, submergence of the New Zealand archipelago reduced the land area to approximately $\sim 20 \%$ of its current size, and together with recent glacial-interglacial cycles, has promoted speciation and regional endemism patterns as well as species range disjunctions (McGlone et al. 2001). Several genera have undergone species radiations in New Zealand, notably the Celmisia, Chionochloa, Ranunculus, Epilobium, Aciphylla, Ourisia genera and the Hebe complex (Scrophulariaceae) (Mark 2012). Consequently, the New Zealand flora is highly endemic at the species level ( $82 \%$ of vascular plant species), but generic endemism 
rates are substantially lower ( 10\%) and there are no known endemic vascular plant families (McGlone et al. 2001, Winkworth et al. 2005).

There are several distinctive features of the New Zealand flora - notably, paucity of annual plants, and high levels of gender dimorphism and small seeds (McGlone et al. 2001). These characteristics reflect dispersal selection and despecialisation upon arrival. For example, the New Zealand alpine flora generally has a high preponderance of white, and infrequently yellow, flowers (Campbell et al. 2010) which are pollinated by generalist insect species belonging to many taxa (Primack 1983). Further, the New Zealand alpine flora encompasses many examples of "insular woodiness" (notably Carmichaelia and Veronica genera), where ancestral herbs that are disproportionately represented in trans-oceanic dispersal events evolve into shrubs and trees (McGlone et al. 2001). Another interesting characteristic of a number of alpine taxa is irregular sexual reproduction (Mark 2012). For example, Chionochloa, Nothofagus and Aciphylla are all masting genera. Conversely, many traits are rare in the New Zealand flora relative to global trends: deciduous leaves, complex or specialised flowers, annual habit, ant dispersal and fire-release germination (McGlone et al. 2001). The climatic history of recent glacial-interglacial cycles has resulted in little specialisation to cold climates. Consequently, tree lines in New Zealand are lower than in other regions (McGlone et al. 2001). As in many alpine systems, the New Zealand alpine flora is strongly structured by climatic factors, especially altitude (Wilson and Meurk 2011). Species with a large spatial distribution tend to have larger altitudinal and climatic tolerances and have better dispersal ability (McGlone et al. 2001). However many species are specialists of habitats or regions (Mark 2012). 


\section{Tongariro National Park}

Tongariro National Park (TNP) is a UNESCO natural and cultural heritage site (Baird 2013) situated on the central volcanic plateau of the North Island of New Zealand $\left(-39.25^{\circ} \mathrm{N}\right.$, $175.58^{\circ} \mathrm{E}$ ). The park encompasses three large volcanoes (Ruapehu, 2793m; Ngauruhoe, 2290m; Tongariro 1986m), which reached their present height during the Pleistocene (Mark 2012). Presently, the volcanoes contribute to significant rain shadow effects (Mark 2012). This volcanic history results in dark sandy-loam and loamy-sand soils with poor drainage (Chapman and Bannister 1990) that are highly porous and prone to erosion (Mark 2012). TNP provides habitat for a diverse native alpine plant community (Halloy and Mark 2003) which is predominantly indigenous to New Zealand (Wardle 1991), with many subspecies occurring exclusively within the park (Chapman and Bannister 1990, Rogers and Leathwick 1996). Native forests within the park are dominated by Nothofagus solandri var. cliffortioides (三 Fuscospora cliffortioides; for taxonomic discussion see: Hill et al. 2015) and the natural treeline occurs at $\sim 1500$ m on Ruapehu (Mark 2012). N. solandri forests were extensive throughout the park before the arrival of humans 1000 years BP (Rogers and Leathwick 1996). However, the realised treeline is significantly lower than the climatic treeline in most parts of the park $(<1300 m$, restricted to the south-west of the park; Rogers and Leathwick, 1996). TNP has a high disturbance history resulting from volcanic activity, erosion and anthropogenic burning (Keesing 1995, Mark 2012). The vegetation of TNP is currently highly heterogeneous, being a product of orographic rainfall patterns, elevational patterns and disturbance (Atkinson 1985). The permanent snowline on Mt Ruapehu is ( 2400m) (Mark 2012).

At low elevations, where forest is absent, scrubland is prevalent, with substantial Dracophyllum spp., Leptospermum scoparium and Phyllocladus alpinus components (Rogers and Leathwick 1996). In more recently disturbed areas, Chionochloa rubra grassland predominates. Above $\sim 1200 \mathrm{~m}$, two vegetative communities are common: those with an intact ash layer, and loose gravel fields where the ash has been eroded (Rogers and Leathwick 1996). The former hosts extensive Gleichenia dicarpa and Empodisma minor bogland, as well as Lepidothamnus laxifolius, Veronica odora, V. tetragona, Halocarpus bidwilli and Epacris alpina, which additionally acquires substantial components of Dracophyllum spp., Podocarpus nivalis 
and $P$. alpinus as the community succeeds to scrubland. In contrast, the gravelfield are susceptible to frost heaves and are typified by sporadic Dracophyllum recurvum, Gaultheria colensoi and Lepidothamnus laxifolius, that consolidate the soil (Rogers and Leathwick 1996). The grasses C. rubra, Rytidiosperma setifolia as well as the moss Racomitrium lanuginosum are common in these communities. The Rangipo desert, located in the east of the park, is typified by 'frost-flat' communities (Smale 1990). This area is susceptible to temperature inversions caused by cold air drainage and pooling, and C. rubra grasslands or Dracophyllum subulatum scrubland are prevalent (Smale 1990). A diverse herb and low stature shrub community is additionally present at all elevations in the alpine zone, including species that are widespread throughout New Zealand, such as Anisotome aromatica, but also many species endemic to the central North Island, such as Ourisia vulcanica (Mark 2012).

\section{Global change in the park}

Higher than global mean rates of anthropogenic climate change have been recorded in parts of New Zealand; TNP is currently $1.5^{\circ} \mathrm{C}$ warmer and drier $(-5 \mathrm{~mm}$ of rain/year) than it was 50 years ago (IPCC 2014). Median annual air temperature on the lowlands of the central volcanic plateau is $9^{\circ} \mathrm{C}$ and decreases with elevation (Chappell 2015). Alpine plant communities in TNP are at particularly high risk from climate change as this alpine habitat is relatively small in extent and is isolated from other alpine areas (Halloy and Mark 2003). The North Island alpine ecosystem comprises of 87 separate areas above $1000 \mathrm{~m}$; with a $3^{\circ} \mathrm{C}$ temperature rise, only 6 would retain conditions allowing the persistence of alpine communities (Halloy and Mark 2003). A growing lack of true alpine areas on the North Island as temperatures rise accords TNP a crucial role in the conservation of alpine biodiversity on the North Island. Other than predictions based on findings in other alpine ecosystems, very little is known about ecosystem responses to climate change in TNP. In New Zealand, it is predicted that up to 300 alpine plant species could be at risk under climate predictions of a $3^{\circ} \mathrm{C}$ increase (Halloy and Mark 2003).

Alongside climate warming, a major driver of ecological change in TNP is Calluna vulgaris (L.) Hull (European heather; Ericaceae) invasion (Chapman and Bannister 1990, Rogers and Leathwick 1996). In fact, New Zealand generally is identified as a future invasion hotspot 
(Sheppard et al. 2016). C. vulgaris was intentionally introduced to the park in 1912 (Rogers and Leathwick 1996); from 1912 to the 1922, multiple intentional introductions and active plantings from a variety of European source populations were made (Keesing 1995). By 1991, C. vulgaris occupied about $1 / 3$ of the surface of the park (Keesing 1995) and occurred in all plant community types from 600 to 1600 m (Chapman and Bannister 1995). C. vulgaris is an evergreen woody dwarf shrub (Watt 1955) native to Europe, with a distribution from Southern Spain to Scandinavia, and from the Azores to the Urals (Rendell and Ennos 2002). A number of C. vulgaris cultivars are protected in Europe, where C. vulgaris is an integral part of culturally important heathland ecosystems that are in decline (Mahy et al. 1999). C. vulgaris is a generalist in habitat selection (Watt 1955). C. vulgaris is also a generalist in its pollinator interactions and is both wind and insect pollinated (Descamps et al. 2015). Further, it forms facultative (Bannister and Norton 1974) ericoid mycorrhizas with generalist fungal partners (Johansson 2001). Finally, although C. vulgaris prefers acidic soils, its edaphic requirements are broad relative to many plant species (Walker et al. 2007, Fühner and Runge 2009). C. vulgaris produces large numbers of small (5-8mm length), bell-shaped, tetramerous flowers in late summer (Chapman and Bannister 1995, Descamps et al. 2015). C. vulgaris is a diploid, cross pollinating plant $(2 n=16)$ (Behrend et al. 2013). The species produces small seeds and can achieve $10^{6}$ seeds $\mathrm{m}^{-2} \mathrm{yr}^{-1}$ (Miller and Cummins 2001, Soons and Bullock 2008). Chloroplast DNA (CpDNA) data as well as allozyme data indicate that $C$. vulgaris populations within its native range are genetically structured (Mahy et al. 1999, Rendell and Ennos 2002). However, genetic diversity in this species may be relatively low (Behrend et al. 2013).

C. vulgaris invasion has driven a decline in many native plant species and may reduce species richness (Keesing 1995). New Zealand's insular flora makes it particularly susceptible to plant invasions generally (Sheppard et al. 2016). In TNP, C. vulgaris has outcompeted the previously dominant tussock grass - C. rubra - in many parts of the park, particularly below 1200m (Rogers and Leathwick 1996). This is partially because conditions in inter-tussock spaces are ideal for seed establishment (Chapman and Bannister 1990). Species that may be particularly vulnerable are inter-tussock species such as dwarf shrubs (Coproma cheesemanii, Copromsa perpusilla and Dracophyllum recurvum) and herbs (Anaphalioides bellidioides and 
Celmisia spectabilis) (Chapman and Bannister 1990). Conversely, some native plant species are favoured by C. vulgaris invasion (Viola filicaulis, Aciphylla sigurrosa, Hydrocotyle novaezealandia var montana, amongst others; Chapman and Bannister 1990). C. vulgaris invasion also affects insect assemblages (Keesing 1995). Ecosystem responses to the invasion of this species are unsurprising given that $C$. vulgaris is known to achieve aboveground biomass of up to $4.8 \mathrm{~kg} \mathrm{~m}^{-2}$ (Keesing 1995). C. vulgaris occupies a large volume of space with densely packed biomass in these communities, and has a more complex above-ground architecture than native species (Keesing 1995). C. vulgaris continues to spread within the park (Chapman and Bannister 1990), particularly east (Rogers and Leathwick 1996) and increasingly out of TNP (Keesing 1995). Management of this species has included the introduction of a biocontrol agent, the heather beetle (Lochmaea suturalis), which reduces C. vulgaris biomass locally (Syrett et al. 2000).

\section{Māori as kaitiaki in the park}

One way in which ecological systems provide value to people is through cultural services, often by sustaining species of cultural significance (Costanza et al. 1997). Ecosystems can be especially important to indigenous peoples with long histories of interactions with the landscape (Gadgil et al. 1993). Consequently, preserving cultural heritage is an important axis of environmental conservation (Satterfield et al. 2013). The importance of cultural preservation in TNP is significant, and the park was initially established on the basis of a gift made by Te Heuheu Tūkino IV Horonuku of Ngāti Tūwharetoa to the Crown (Baird 2013). Approximately 2640 ha of land around the summits of Tongariro, Ngauruhoe and Ruapehu were ceded in 1887; the original gift to the Crown was expanded to include 80,000 ha (Baird 2013). TNP holds spiritual, cultural, and ancestral meaning for local tangata whenua (the people of the land). The iwi (tribes) Ngāti Rangi and Ngāti Tūwahretoa are kaitiaki (guardians) over the park (Baird 2013) and have a long history of participation in environmental conservation in the park. In New Zealand, co-management is a legal requirement (Taiepa et al. 1997). In accordance with the Department of Conservation management plan, conservation in TNP should “...engage in regular, active, and meaningful consultation with iwi" (The Team of Tongariro/Taupō Conservancy 2006). 
Much of my thesis work was carried out in collaboration with Ngāti Rangi (a southern Ruapehu iwi), the University of Copenhagen (Denmark), and the Department of Conservation. In 2016, we jointly secured the Ministry of Business, Innovation and Employment - Vision Mātauranga Capability Fund to develop new expertise and tools to address significant challenges to conservation management. Our collaboration aimed to restore our capacity to act as kaitiaki of TNP by strengthening the connections between Ngāti Rangi and the science underpinning conservation management in the park. Our research had three goals: 1) to increase the accessibility of Mātauranga Māori (traditional Māori knowledge) and scientific knowledge of taonga tipu (culturally important plant species) to all people. This was achieved locally through effective outreach with the community, and globally through the creation of new web-based materials; 2) using species distribution modelling techniques, we generated new understanding of how taonga tipu will cope with multiple drivers of ecosystem change, identifying species at increased risk of extinction. This information is critical for the development of effective conservation management plans for the kaitiakitanga (guardianship) of the taiao (the environment) ; 3) through hands-on collaboration and involvement, the project built expertise among individual Ngāti Rangi members, strengthening connections between Ngāti Rangi and conservation science and management through building capacity for Ngāti Rangi to contribute to effective kaitiakitanga of taonga (treasures) for the benefit of all New Zealanders. 


\section{Approaches to understanding global change impacts on ecosystems}

Many lines of evidence are used to ascertain the responses of ecosystems to global change drivers (Halloy and Mark 2003, Parmesan 2006). Here, I discuss four relevant methods which are used to empirically study ecological responses to global change - the use of longterm monitoring, field surveys, manipulative experiments and spatially explicit modelling techniques. I focus on the use of these technique in the study of climate change and species invasions. I conclude with the role of reviews and meta-analyses in understanding ecosystem responses to global change.

\section{Historical data}

The comparison of current and historical data is an important line of evidence for ecological responses to global change drivers (Parmesan 2006) because many ecosystem processes operate over long time scales (McLaren and Turkington 2011). For example, Brusca et al. (2013) re-surveyed an established elevational transect after five decades and measured a dramatic response of the plant community to recent climatic change. Long term monitoring, through the use of photo points and permanent plots also provides ample evidence for change in natural ecosystems (Halloy and Mark 2003). Alternatively, the use of herbarium records can allow researchers to determine shifts of plant species distributions or phenology (Gallinat et al. 2018). However, the use of long-term datasets is hampered by data availability. Further, long term data are particularly skewed towards Northern Hemisphere ecosystems and small geographic scales, limiting their applicability to questions at larger spatial scales (Parmesan 2006). Finally, long-term data collections and the comparison of current and historical data provides correlative evidence of ecosystem responses to drivers of change, however changes of ecosystems may reflect responses to other sources of disturbance (Halloy and Mark 2003).

\section{Observational studies}

The response of plant communities to global environmental change is often investigated by surveying the natural biological variability in ecosystems (Parmesan 2006, Hector and Loreau 2007). For example, the use of abiotic gradients with statistical tools to understand the responses of species to climatic conditions has a long-standing history in ecology (Grinnell 1917, 
Whittaker 1967, Callaway et al. 2002, Körner 2007, Michalet et al. 2014). Observational studies have the benefit of realism relative to more reductionist experimental approaches. Further, observational studies permit the investigation of processes which may occur at spatial and/or temporal scales which are irreplicable experimentally. Comparisons of vegetative communities along elevational gradients are therefore often used in lieu of climate manipulations (Michalet et al. 2014). Elevational gradients result in consistent abiotic variability over small geographic areas, enabling studies comparing the ecology of species or communities under different environmental stress conditions (Körner 2013). For example, the nature of species interactions along elevational gradients underpins our understanding of the role of competitive and facilitative interactions in determining patterns of species coexistence and diversity (Callaway et al. 2002). Spatial co-occurrence of species along elevational gradients is used to determine the nature of plant-plant interactions under variable abiotic conditions (Dvorsky et al. 2013, Grassein et al. 2014, Kikvidze et al. 2015). Further, co-occurrence can inform our understanding of plant community dynamics; for example, Spasojevic and Suding (2012) inferred multiple community assembly processes from functional diversity patterns. Patterns of plant species cooccurrence are also routinely used to estimate niche width (Manthey and Fridley 2009) and used to infer the capacity of species to adapt, genotypically or phenotypically, to climate change (Grassein et al. 2014). Ecosystem function is also analysed along abiotic gradients to examine its sensitivity to abiotic conditions. For example, plant-pollinator interactions vary with environmental stress (Theobald et al. 2016).

Many gradients other than elevation are used in conjunction with surveys to infer ecosystem responses to drivers of global change. For example, the comparison of the numbers of invasive plant species in reserves with different characteristics (Timmins and Williams 1991), with variable climatic or human disturbance conditions (Gallardo et al. 2015), or in proximity to known vectors of transport (Joly et al. 2011) allow researchers to ascertain the relative importance of factors facilitating plant invasions. Likewise, surveys of native plant communities are conducted at invasion fronts to deduce the impact of invasive species on native communities. These surveys typically compare paired plant communities that are invaded and uninvaded (Powell et al. 2013). Data on species presence is most commonly collected in 
vegetative surveys; abundance data is more informative in many cases but is scarcer (Ransijn et al. 2015, Bradley et al. 2018).

\section{Experimental approaches}

Manipulative experiments at small scales are a powerful way of investigating mechanisms of ecosystem response to global change (Halloy and Mark 2003). Field experiments reflect a trade-off between keeping all confounding factors constant, thereby unambiguously demonstrating ecological mechanism, and the ecological realism of non-manipulative experiments (such as vegetative surveys; Hector and Loreau 2007). In such experiments, global change drivers are simulated in various ways and are compared to control communities. For example, Arroyo et al. (2013) used a field warming experiment to show that the phenotypic plasticity of flowers under altered temperature conditions allowed plants to maximize their pollination opportunities. Passive Open Top Chambers (OTC) are a commonly implemented experimental methodology used to simulate climate warming; the response of many aspects of plant ecology can be monitored within OTCs (Marion et al. 1997, Arroyo et al. 2013). The OTC approach has been criticised for underpredicting the magnitude of response (Wolkovich et al. 2012). However, OTCs remain an important research tool in global change ecology (Wolkovich et al. 2012). OTCs are less disruptive and expensive than alternative methods of temperature manipulation (Marion et al. 1997). As with climate change, the impact of invasive species can also be deduced using field experiments. Species removal experiments are particularly useful in exploring species interactions (Hector and Loreau 2007) because they can be used in ecosystems dominated by perennial species where artificial communities are difficult to create (McLaren and Turkington 2011). Most commonly, the magnitude of species interactions is deduced from biomass compensation following the removal of a species or functional group of interest. Strong biomass compensation responses in a plant community following the removal of functional groups illustrate the importance of species interactions with those functional groups in structuring ecosystems (McLaren and Turkington 2011). Species removal experiments have also been used to interpret the role of biodiversity in the functioning of ecosystems (Callaway et al. 2002, Wardle and Zackrisson 2005). Many manipulations other than removal are used to investigate ecological responses to invasion. For example, the addition of invasive 
flowers and monitoring the effect on the pollination success of native species can indicate the potential ecological consequences of invasions for the reproduction of native plants (Chittka and Schurkens 2001, Brown et al. 2002). Similarly, the removal of pollinator access to flowers can illustrate the relative importance of pollination services and other sources of sexual reproductive failure (Da Silva et al. 2013).

Manipulative experiments have limitations. For example, experiments can inadvertently create unintended consequences that may precipitate to potentially unwanted experimental artefacts (Marion et al. 1997). Additionally, responses of ecosystems may vary with the duration of the manipulation (Chapin and Shaver 1996, McLaren and Turkington 2011), reflecting the natural lags in some ecosystem responses. Consequently, long-term experiments are particularly important (Laliberte et al. 2013). Despite their limitations, field experiments are a key tool in testing mechanisms in complex ecosystems and the interpretation of experimental results drives many aspects of ecological theory (Loreau 2000). The strength of field experiments can be bolstered by replicating experimental design at multiple sites (Hector and Loreau 2007).

\section{Modelling}

Forecasting the responses of ecosystems to global change is an important task for global change biologists and conservationists (Clark et al. 2001). For this reason, spatially explicit models, and in particular Species Distribution Models (SDM), which allow for predictions of ecological conditions resulting from global change are an area of rapid development in the literature (Beaumont et al. 2008, Fordham et al. 2014). Predictive distribution models and techniques are developing rapidly to produce more accurate predictions of species' distributions (Caughley et al. 1988, Guisan and Thuiller 2005, Elith et al. 2006). This growing area of study is galvanised, in part, by the increasing availability of spatially explicit species occurrence data and high-resolution environmental data. SDMs derive from ecological niche theory and gradient analysis (Franklin 1995). SDMs have many applications and have been used to predict the future range expansion of invasive species (Mainali et al. 2015) to quantify the risk of plant invasion into mountain ecosystems (Petitpierre et al. 2016) or to identify which 
species will be vulnerable to climate change (Watling et al. 2013). SDMs consistently predict high species turnover and significant reductions in plant diversity under future climate scenarios (Engler et al. 2011) as well as alterations of invasive species' distributions (Bellard et al. 2018).

There are two common approaches to the construction of SDMs: models are generally either correlative or mechanistic (Buckley et al., 2010). Correlative approaches (such as climate envelope models) create statistical relationships between current distributions and environmental conditions (Lavergne et al. 2010, Watling et al. 2013). Climate envelope models are prolific (Araújo and Guisan 2006, Nogués-Bravo 2009) and typically require data on the presence and absence of a species and a spatially explicit description of environmental conditions over the corresponding geographical range (Lavergne et al. 2010). They do not factor in other variables that could delineate species distributions, such as habitat availability, fragmentation and competition and often omit soil characteristics which tend to vary at finer scales (Watling et al. 2013). This is, in part, because historically climate has been regarded as the primary variable determining species distributions, although this assumption is increasingly questioned (Wisz et al. 2013). Moreover, climate envelope models are static and probabilistic in nature; they assume that correlations between environmental variables are constant, and that variables determining the range of species are independent (Buckley et al. 2010). Additionally, they assume that species' ranges are in equilibrium with current conditions (Nogués-Bravo 2009, Dormann et al. 2012). These assumptions are particularly problematic when making dynamic predictions and it is expected that relationships between environmental variables will be violated when models are used to extrapolate beyond current conditions (Buckley et al. 2010).

In contrast to correlative methods, mechanistic models are often more complex as they explicitly model all processes important in determining species distributions (Dormann et al. 2012). Mechanistic models create explicit mathematical descriptions of the relationship between organism fitness and environmental conditions, typically derived from empirical data (Buckley et al. 2010). This approach is theoretically more appropriate for dynamic predictions of species ranges under future climates (Buckley et al. 2010). However mechanistic models may 
also inaccurately predict species range shifts under future climate change scenarios, because miscalibration of key parameters caused by imperfect understanding of ecological process will lead to poor predictions (Dormann et al. 2012). For example, the fundamental constraints limiting species distributions identified at one location may not be important along the entire species' range (Buckley et al. 2010), or important predictors may be overlooked (Dormann et al. 2012). More recently, hybrid models including correlative and mechanistic components are emerging (Peknicova and Berchova-Bimova 2016). Notably, many SDMs entirely omit the role of species interactions, despite their importance in shaping species distributions (Wisz et al. 2013) and hybrid models could account for this. For example, Joint Species Distribution Models (JSDM) partially account for species interactions by explaining the residual variance of a climate model with pairwise species co-occurrence data (Pollock et al. 2014); their application is increasing in SDM studies.

Numerous algorithms are used in SDM and can yield substantially different results even where the same training data are used (Araújo and Guisan 2006). In some cases, such projections from alternative models can vary to such a significant degree that their utility is compromised (Araújo and New 2007). Nevertheless, evaluations of modelling techniques have not identified a singular superior approach. Consequently, ensemble models, that integrate multiple computations across a number of initial conditions, model classes, parameters and boundary conditions, have been put forward as a solution (Araújo and New 2007, Thuiller et al. 2009). These combined approaches integrate the 'wisdom of the commons' - consensus forecasts tend to yield lower error rates than any of the individual forecasts of which it is composed (Araújo and New 2007, Thuiller et al. 2009, Sheppard et al. 2016). SDM is a statistical tool that is developing rapidly. More sophisticated SDM techniques are leading to increasing utility and predictive power in species and ecosystem models (Elith et al. 2006, Heikkinen et al. 2006).

\section{Meta-analyses}

Meta-analyses and reviews allow the field of global change to explore universalities or highlight emerging research avenues (Brook et al. 2008, Schweiger et al. 2010, Wu et al. 2011). 
Meta-analyses identify commonalities in ecosystem responses to drivers of global change among studies conducted globally. For example, Parmesan and Yohe (2003) applied metaanalytic techniques to estimate that $59 \%$ of 1598 species exhibited measurable phenological or distributional responses to recent climate change. Alternatively, meta-analyses can identify discrepancies in experimental results that are used to form further hypotheses (Bunn et al. 2015). For example, Schweiger et al. (2010) identified common effects of interactions between plant invasion and climate change on plant-pollinator interactions in recipient ecosystems and postulated potential impacts on plant-pollinator networks. In emerging disciplines such as SDM, where studies use many different methodologies and a singular best approach has not been found (Araújo and New 2007), reviews may identify best practice methods for generating reliable forecasts of global change effects (Jimenez-Valverde et al. 2011).

\section{Research objectives and outline of this thesis}

My thesis work explores the interactive effects of global change drivers on community structure and ecosystem function using TNP as a model ecosystem. My work is novel in that it focuses on the direct and interactive effects of climatic warming and plant invasion using observational, experimental, and modeling approaches (Figure 1.1). Recognizing that climate change may directly affect native plants in TNP, but may also augment the effects of $C$. vulgaris, my approach is to measure the impact of climate on both native ecosystem structure and function as well as on C. vulgaris, and to explore changing interactions between native species and $C$. vulgaris under different abiotic or landscape conditions (Chapters 2-4). In Chapter 5, I explore the interaction between plant invasion and human disturbance to determine the relative roles of biological filters and anthropic facilitation on the invasion of $C$. vulgaris across New Zealand. 


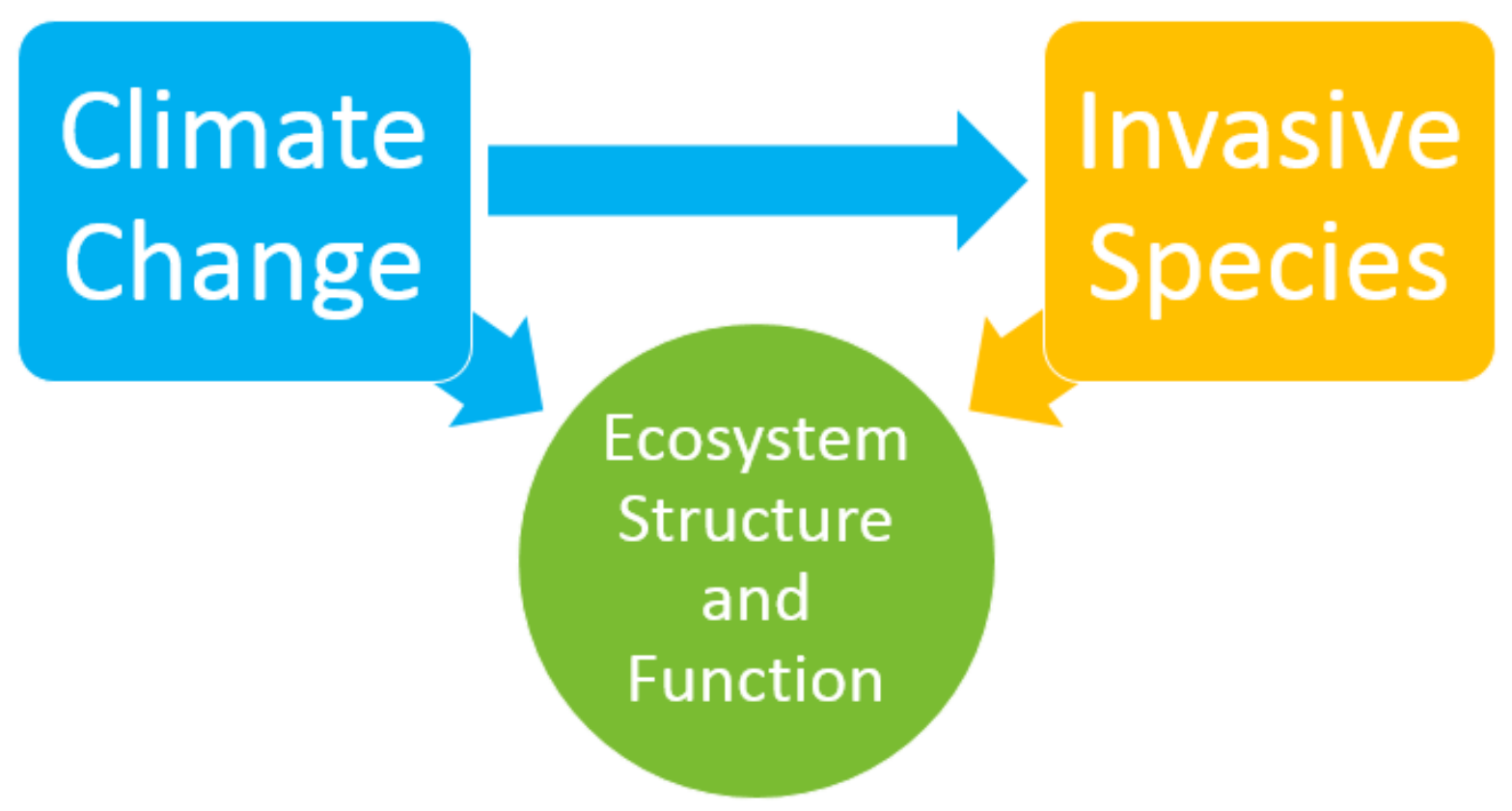

Figure 1.1: Theoretical framework guiding this thesis. Drivers of global change may interact, leading to non-linear responses of ecosystems. Climate change affects aspects of ecosystem structure and function directly, but also influences invasive species, thereby exacerbating or diminishing the effects of invasive species that are recognized under current climatic conditions.

My research addressed four inter-related questions:

Chapter 2: Does the phenology of co-occurring native and invasive plants respond to climate warming differently and, if so, what are the consequences of species-specific phenological responses for the sexual reproduction of native species?

Chapter 3: How does native alpine plant species richness respond to plant invasion at multiple spatial scales and is this response modulated by environmental stress? 
Chapter 4: What are the consequences of climate change and density-dependent competitive interactions with C. vulgaris on the distribution and density of taonga tipu (culturally important plant species) in the park?

Chapter 5: Are other areas of New Zealand suitable for the invasion of $C$. vulgaris and why has this species not spread to those areas?

I answered these questions using a variety of experimental, observational and modelling approaches. I predicted the responses of individual species, plant communities and ecosystem function.

In Chapter 2, I paired manipulative experiments to probe two mechanistic pathways through which $C$. vulgaris invasion and climate warming may alter phenology and reproduction of native plant species. In the first, I tested how experimental warming $\left(+1.7^{\circ} \mathrm{C}\right)$ modulates the flowering phenology of the plant community and how this affects flowering overlap between a focal native (D. subulatum) and C. vulgaris. In the second experiment, I explored how variation in the ratio of native to invasive flowers, and the overall quantity of floral resources in the landscape, affected the reproduction of $D$. subulatum. I hypothesised that the flowering overlap of $D$. subulatum and $C$. vulgaris flowering would increase under manipulative warming, given that invaders typically exhibit greater phenological plasticity than native species. Further, I hypothesised that the sexual reproduction of $D$. subulatum would decrease in landscapes dominated by $C$. vulgaris flowers, but that this effect would depend on total floral density in the landscape. If correct, these hypotheses together would suggest that under warmed climates, I would expect greater impacts of $C$. vulgaris on the sexual reproduction of $D$. subulatum in some landscapes.

In Chapter 3, I investigated the impact of plant invasion on native alpine vascular plant species richness at two spatial scales and on the slope of the Species Area Relationship $(\mathrm{SAR} ; \mathrm{z})$. I assessed the consistency of this impact along an environmental stress gradient. I hypothesized that the magnitude of both positive and negative effects of invasion on plant richness at the $1 \mathrm{~m}^{2}$ scale would increase with invader biomass, with the exception of positive interactions at very high invader biomass. Further, I hypothesized that $z$ in low stress 
environments would increase monotonically with invader canopy cover, whereas in high stress environments the impact of invader canopy cover should be unimodal: moderate canopy cover should be associated with higher $z$ and dense canopy cover with a lower $z$ compared to uninvaded communities.

In Chapter 4, I developed a novel hierarchical modelling framework which integrates pairwise density-dependent effects with an invader and couple my modelling work with a largescale field study. I applied this to a native species, C. rubra in an alpine region of New Zealand. I used occurrence data to model the distribution of the native species and of the invasive species C. vulgaris, under both current and future climate scenarios. I investigated the density dependence of these species using quadrats which spanned both elevational and $C$. vulgaris invasion density gradients. I identified C. vulgaris density thresholds above which C. rubra density was significantly reduced. Next, I predicted the proportion of $C$. rubra distribution which overlaps with environmental conditions that may support densities of $C$. vulgaris that are above this threshold (the 'impact niche') under current and future climate scenarios. My models predict a contraction of suitable space for $C$. rubra under future climates. Chapter 4 describes the response of one of the five modelled taonga tipu - Chionochloa rubra. The results for the remaining four species are in Appendix 4. While the results for all five species are interesting, I limited the scope of Chapter 4 in order to streamline this work for an initial publication of the modelling methodology; I intend to publish the results in Appendix 4 as a follow up publication.

In Chapter 5, I used SDM to explore the potential for $C$. vulgaris to expand its invasive range on the North and South Islands of New Zealand. C. vulgaris was introduced on the North and South Islands of New Zealand, with notably different outcomes. On the North Island, C. vulgaris is invasive and is expanding its range; on the South Island, C. vulgaris grows in several isolated populations where it has been present, in some places, for over a century but is not spreading at the high rates observed on the North Island. Contrasting this variable success on the two islands - that differ in the extent and intensity of human disturbance - can illustrate the relative importance of environmental filters and anthropic facilitation in determining the invasive success of invasive plants in novel ranges. I built climate envelope models and paired these with maps of other factors known to affect the spread of invasive plants - soil $\mathrm{pH}$, 
presence of mycorrhizal mutualists, vegetation type and the extent and intensity of human impact on the landscape. I hypothesised that that the majority of both islands is climatically, edaphically and ecologically suitable for $C$. vulgaris. I additionally hypothesised that $C$. vulgaris populations on both islands are associated with medium to high levels of human disturbance, despite substantial differences in the prevalence of human disturbance between the two islands. 
2 Climate change and invasion synergisms may cause decline of native plant reproduction through species-specific phenology

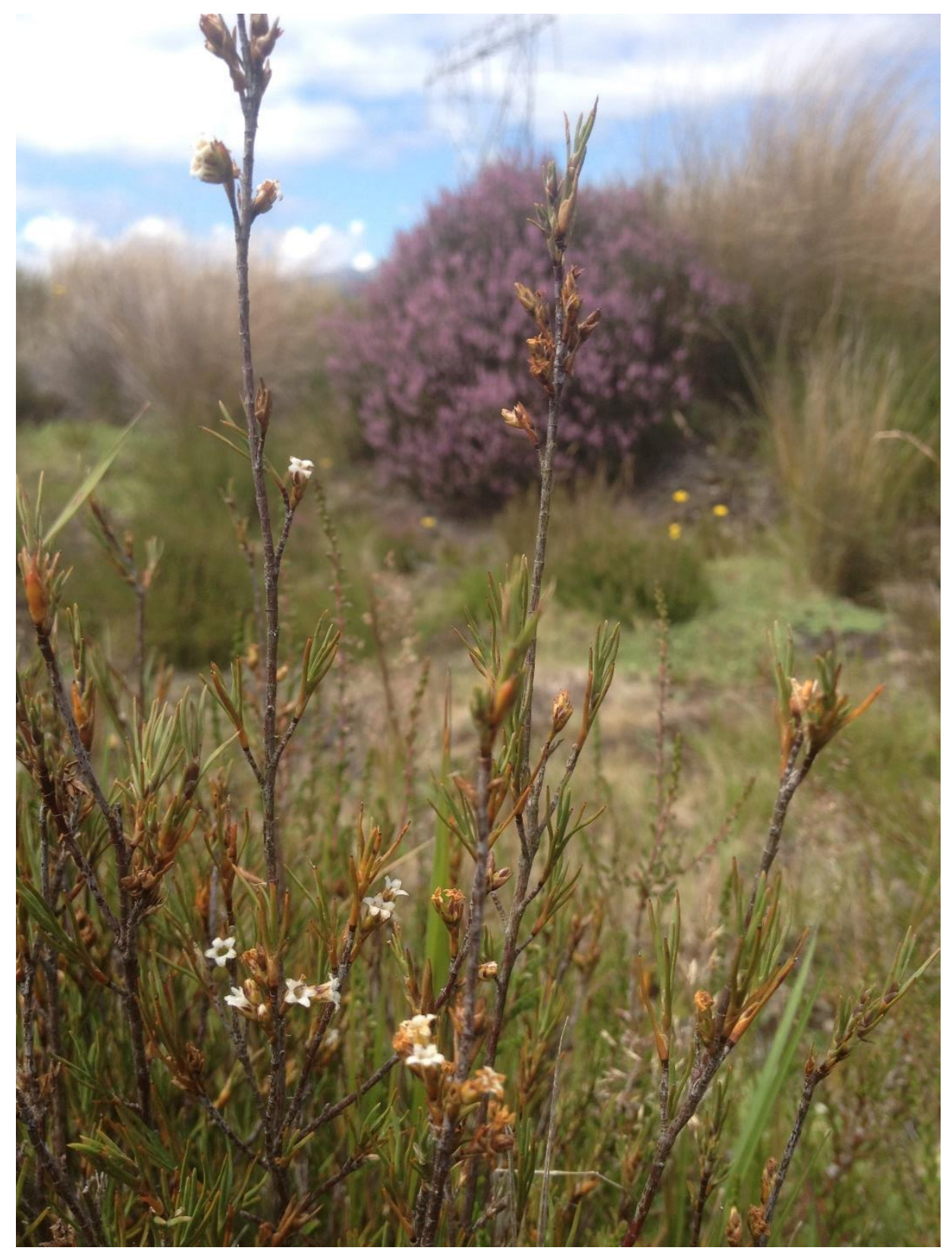

Dracophyllum subulatum flowers (in the foreground) compete for insect pollinators with the invasive species Calluna vulgaris (in the background).

Giejsztowt, 2018 


\section{Introduction}

Climate warming and species invasions are important drivers of global environmental change. Both drivers impact ecosystem structure and function directly (Chapin et al. 2000, Mack et al. 2000, Tylianakis et al. 2008, Vila et al. 2011). Global change drivers can also interact, resulting in an exacerbation or buffering of their direct effects on ecosystems (Tylianakis et al. 2008, Schweiger et al. 2010, Yang and Rudolf 2010, González-Varo et al. 2013, Sparks and Menzel 2013, Fox et al. 2014). For example, extinctions attributed to global warming are often driven by synergisms with invasion (Brook et al. 2008). These, often negative, interactions are particularly striking when they impact important ecosystem functions such as pollination (Hoover et al. 2012, González-Varo et al. 2013). Animal pollination, which is essential for crop production as well as the reproduction of most angiosperms (Burkle et al. 2013, González-Varo et al. 2013), may become limiting because pollinators are threatened with extinction (Knight et al. 2018). It is predicted that a loss of pollination may lead to declines in plant populations (Potts et al. 2010, Burkle et al. 2013). However, the effect of interacting global change drivers on the pollination of plants is poorly understood (Potts et al. 2010, Hoover et al. 2012, González-Varo et al. 2013, Fox et al. 2014). To date, no study has experimentally investigated the impact of climate change and plant invasion on the phenology and reproduction of native plants.

Changes in phenology are a prominent response of plants to climate change, both locally and globally (Parmesan and Yohe 2003, Hoover et al. 2012, Gallinat et al. 2018). Flowering phenology is important because it is related to plant fecundity (Godoy et al. 2009). Flowering phenology is determined by genetics as well as a suite of cues, such as day length, soil moisture, and air temperature (Godoy et al. 2009, Burkle et al. 2013, Davies et al. 2013). Further, phenology may vary in its response to climate change depending on whether a species flowers early or late in the season (Ji et al. 2017). Shifts in flowering phenology can be speciesspecific (Gallinat et al. 2018). Consequently, climate change may have various effects on plantpollinator interactions. For example, changes in flowering phenology are likely to result in spatial or temporal asynchronies between interacting mutualists (Parmesan and Yohe 2003, Yang and Rudolf 2010, Hoover et al. 2012, Kharouba et al. 2018, Knight et al. 2018). 
Alternatively, flowering phenology may augment landscape nutrient economies, which are known to alter pollinator behaviour (Hoover et al. 2012). Altered plant-pollinator interactions may lead to pollen limitation for some plant species (González-Varo et al. 2013), affecting their reproductive success (Gallinat et al. 2018). Invasive plants typically display more phenological plasticity than native plants (Gallinat et al. 2018) which may be important if climate warming alters the extent of flowering overlap between native and invasive species.

Invasive plant species can readily integrate into existing plant-pollinator networks and modify floral resources in landscapes (Potts et al. 2010, Drossart et al. 2017) with sometimes positive (Morales and Traveset 2009, Knight et al. 2018) but generally negative consequences for native plant reproduction (González-Varo et al. 2013). Prolific flower production, which is a characteristic of many invasive species, may create interspecific competition for pollinators (Chittka and Schurkens 2001, Brown et al. 2002, González-Varo et al. 2013), particularly where the flowers of invasive species have high nutritional value or are easily foraged (Drossart et al. 2017). For example, Chittka and Schurkens (2001) simulated shifts in floral abundance between an invasive and a native plant, which resulted in a decline in native plant seed set relative to "uninvaded" patches. Competition for pollinators may directly affect the fitness of natives by reducing pollinator visitation rates and/or pollination quality (Brown et al. 2002, Mitchell et al. 2009). Conversely, invasive species may enhance pollination services to native plant species by providing higher resource stability for pollinating insects (Potts et al. 2010, Knight et al. 2018). Species with showy floral displays or high rewards may increase the attractiveness of a vegetation patch, thereby improving pollination services to other plant species within the patch. Studies investigating competition for pollination should therefore account for landscape context (Ekroos et al. 2015, Knight et al. 2018).

Few studies investigate the effect of more than one driver of global change on the pollination of plants, and the interactive effects of multiple drivers remain poorly understood (Tylianakis et al. 2008, Schweiger et al. 2010, Hoover et al. 2012, González-Varo et al. 2013, Knight et al. 2018), with empirical data especially lacking (Hoover et al. 2012). Here, we use a series of experiments to examine the effects of warming and plant invasion on the pollination of a native plant species. Our experiments were designed to elucidate two mechanistic 
pathways for these effects: plant phenology and reproduction. Firstly, we tested how the flowering phenology of native and an invasive species responded to experimental warming in the field over two summer seasons - after two and three years of treatment. We hypothesised that the invasive species would show more plasticity in its phenology than native species of the same growth form, and that this would lead to altered overlaps in the flowering periods of the invasive and some of the native species. Secondly, we tested how the floral landscape (the ratio of native to invasive flowers as well as total landscape floral density) affected the reproduction of a native species. We hypothesised that lower seed masses for native species would occur in landscapes dominated by invasive flowers, and that this effect would be greater in rich floral landscapes. 


\section{Methods}

\section{Study site and species}

We investigated the impacts of climate warming and plant invasion on the pollination of a native plant species in an alpine community in Tongariro National Park, New Zealand ($\left.39.25^{\circ} \mathrm{N}, 175.58^{\circ} \mathrm{E}\right)$. The park is both a natural and cultural UNESCO World Heritage Site (Baird, 2013) and hosts diverse alpine plant communities with high endemism (Chapman and Bannister 1990, Wardle 1991, Rogers and Leathwick 1996, Halloy and Mark 2003). The national park encompasses three large volcanoes resulting in dark sandy-loam and loamy-sand soils (Chapman and Bannister 1990). The climate of Tongariro National Park is changing at higher than the global mean rates and is currently $1.5^{\circ} \mathrm{C}$ warmer and receives $5 \mathrm{~mm}$ less precipitation per annum than it did 50 years ago (IPCC 2014). Median annual air temperature on the lowlands of the central volcanic plateau is $9^{\circ} \mathrm{C}$ and decreases with elevation (Chappell 2015). Tongariro National Park is invaded by Calluna vulgaris (L.) Hull (Ericaceae) which drives a decline of native and endemic plant species, and affects insect assemblages (Wardle 1991, Keesing 1995). C. vulgaris was intentionally introduced to the park in 1912 (Rogers and Leathwick 1996), occupying approximately one-third of the park by 1991 and continues to spread (Keesing, 1995).

C. vulgaris produces large numbers of small (5-8mm length), bell-shaped, tetramerous flowers (Descamps et al. 2015) that provide nectar of high nutritional value (Drossart et al. 2017). C. vulgaris flowers that are utilized by a diverse range of insect species (Descamps et al. 2015, Drossart et al. 2017). C. vulgaris is a late flowering species with a peak flowering period extending to late March in New Zealand. Moreover, its flowering phenology is plastic, responding both to interannual climatic variability and elevational gradients within Tongariro National Park (Chapman and Bannister 1995). The invertebrate pollinator community of the park, like that of New Zealand more generally, is dominated by generalists (Primack 1983, Keesing 1995) . C. vulgaris is not pollen-limited in Tongariro National Park, which may reflect integration into the pollination network, or its capacity to self-pollinate, or both (Murphy and Robertson 2000, Thompson and Knight 2018). C. vulgaris has the greatest mean number of 
flowers per individual plant where it has invaded, and dominates the plant canopy, traits associated with strong competitors for pollination (Chittka and Schurkens 2001).

Dracophyllum subulatum (Epacridaceae) is endemic to the North Island of New Zealand and occupies similar edaphic and climatic environments as C. vulgaris (Smale 1990). D. subulatum is often dominant in 'frost-flat' communities, which are assemblages of alpine species typical of the lowlands of Tongariro National Park (Smale 1990). Its ubiquity is maintained by a low frequency natural fire regime and unseasonal frosts related to temperature inversions (Smale 1990). Large portions of the limited geographic range of this habitat structuring species is undergoing substantial invasion by $C$. vulgaris. This justifies an investigation of the vulnerability of $D$. subulatum to $C$. vulgaris invasion. The flowering phenology of Dracophyllum species is known to respond to inter-seasonal temperature variability (Primack 1980). To our knowledge, no study has investigated pollen limitation of native plant species in C. vulgaris invaded areas of New Zealand.

\section{Manipulative warming experiment}

To investigate the effect of climate on flowering phenology, we utilised a manipulative warming versus ambient temperature experiment ( $n=8$ per treatment) which was established in 2014 on the Rangipo desert, in a frost-flat community of which D. subulatum is a large component, and that is undergoing invasion by $C$. vulgaris. The experiment uses hexagonal open top chambers (OTC; $2.3 \mathrm{~m}^{2}$ ) to increase summer air and soil temperatures; $2 \mathrm{~m} \times 2 \mathrm{~m}$ control plots are also maintained. To confirm the warming treatment effect, we installed temperature loggers (iButton ${ }^{\circledR}$, Maxim Integrated) $5 \mathrm{~cm}$ above the soil surface in all plots. These recorded temperatures at 90 min intervals for the duration of each growing season (NovemberMarch). Over the 2016 and 2017 summers, phenological events were monitored for all vascular species in all plots. Flowering was observed fortnightly for the duration of the growing period in each plot. We recorded first flowering date for each species over the 2016 and 2017 growing seasons. In 2017, we additionally recorded categorical estimations of flower number for each species in each plot. The categories were 0, 1-10, 11-50, 51-100, 101-200, 201-500, 500+. Measurements at each plot were made for four minutes by two people. We estimated the vegetative percentage cover of each species for each plot annually. 


\section{Pollination landscape trials}

To test how the floral landscape (the ratio of native to invasive flowers as well as total landscape floral density) affected the reproduction of $D$. subulatum, eleven pollination trials were completed in 2018, which were restricted to the same habitat type as the warming experiment (early to middle successional communities on the Rangipo desert). The locations and timing of the trials were selected based on two criteria. Firstly, we selected locations for the trials (hereafter "pollination landscapes") that spanned a wide range of flower densities of C. vulgaris and native species. We sought to represent four pollination landscape types in our dataset: (1) high native flower number, high invasive flower number, (2) high native flower number, low invasive flower number, (3) low native flower number, high invasive flower number, and (4) low native flower number, low invasive flower number. We utilized pollination landscapes which varied in floral density due to differences in floral patterns in both time and geographic space (Supplementary Figure 1). All concurrently running pollination trials were a minimum of a $500 \mathrm{~m}$ distance apart, and individual plants within trials were a minimum of $50 \mathrm{~m}$ apart. Secondly, across the pollination landscapes, we sought to maximize the proportional abundance of $D$. subulatum relative to all other native species. This ensured that sufficient conspecific pollen was available to $D$. subulatum across the gradient of pollination landscapes. Within each trial, nine $D$. subulatum plants were selected for study. The nine plants were allocated randomly to one of three treatments: "natural pollination", in which flower buds were not manipulated; "pollinator exclusion", in which pollinators were excluded from flower buds using fine-mesh bags; and "hand pollination" in which pollinators were excluded from buds using fine-mesh bags and flowers were pollinated by hand once these had opened. Eight to ten flower buds per plant received these treatments, dependent on availability.

We monitored flower buds daily until the majority had blossomed, 1-3 days. Labeled flower buds that did not blossom were removed from the experiment in all treatments. We measured the height and diameter of every plant. We collected pollen from the flowers of $20 D$. subulatum plants growing outside of the experiment with a paint brush and applied this to the stigmas of flowers within the "hand pollination" treatment. To quantify the pollination landscape for each plant receiving the "natural pollination" treatment, we used four $25 \mathrm{~m}$ long 
transects. Transects originated from the experimental plant and extended in each cardinal direction. The distance from origin, species identity, and abundance of flowers within $20 \mathrm{~cm}$ of either side of the transect was then recorded. We pooled yellow-flowered members of Asteraceae (Taraxicum and Pilosella) into a single category and counted flower heads rather than individual flowers. Flower abundance was recorded using categorical estimations as above. After approximately six weeks, we collected the seeds from all labelled flowers on each plant. We dried the seeds at room temperature until they were a constant weight and then recorded.

\section{Statistical analyses}

All analyses were performed in R version 3.3.1 (R Development Core Team, 2016). To confirm the warming treatment effect, we used a linear mixed effects model to model air temperature, using the "Ime" function in the package "nlme" (Pinheiro et al. 2018). We set treatment as a fixed effect and time of day as a random effect. As expected, OTCs warmed air

temperature in the treatment plots $\left(F_{(1,5819)}=689.33, p<0.001\right)$. Yearly mean air temperatures in OTC plots were $1.73^{\circ} \mathrm{C}$ higher than in control plots (Supplementary Figure 2). To test whether C. vulgaris has stronger flowering phenology responses than native species including $D$. subulatum, we examined the response of first flowering and peak flowering to experimental warming. We included all plant species that occurred across a minimum of two plots in each treatment. To account for natural variation in species' cover across our experiment, we used floral density (the count of flowers divided by the vegetative cover for each species within the plot, in units of flowers $\mathrm{m}^{-1}$ ) rather than absolute flower number in all analyses. We tested for growth form-specific first flowering and peak floral density responses to warming using linear mixed effect models, with treatment as a fixed effect and plant species as a random effect. We tested for significant differences in first flowering and peak flowering density in D. subulatum and $C$. vulgaris using Welsh t-tests. Next, we tested for flowering overlap between $D$. subulatum and $C$. vulgaris. We plotted the relationship between time of observation and average $D$. subulatum flower density and then calculated the total area under the curve using the trapezoid formula, for experimentally warmed and control plots (Atkinson 1989). We then 
calculated area for only the proportion of each curve where $C$. vulgaris flower densities were high (> 1000 flowers $\mathrm{m}^{-1}$ ).

For our second experiment, we evaluated the effect of pollination landscape on $D$. subulatum seed weight using one-way ANOVA, followed by a post-hoc Tukey's test to deduce pair-wise differences among the three pollination treatments. To meet the assumption of normality we log-transformed seed weights. To account for individual variation in seed weight among plants we modelled seed weight in the hand pollination treatment as a function of the height, diameter and number of flowers on each plant and their interactions. Non-significant predictors were removed from the model in a stepwise fashion. Of the three morphological measurements taken (plant height, plant diameter and number of flowers on the plant), only plant height was a significant predictor of $D$. subulatum seed weight $(F=3.771, d f=2,209, p=$ 0.02). To account for the effect of plant height on seed weight, the slope of this relationship was used to correct $D$. subulatum seed weight in the subsequent regression model (hereafter, we refer to these values as "corrected $D$. subulatum seed weight"). We assessed the effect of floral landscape on corrected $D$. subulatum seed weight in the natural pollination treatment. We applied linear mixed effects models to examine the response of corrected $D$. subulatum seed weight to landscape floral density and the proportion of native flowers in each community. We set latitude and date of the trial as random effects to account for the nonindependence of plants within landscapes. Finally, to ensure that sufficient conspecific pollen was available to $D$. subulatum, we calculated $D$. subulatum flowering density in the pollination landscape and recorded the distance between the focal plant and its closest conspecific. All datasets and R code available at www.github.com/jgiejsztowt.

\section{Results}

\section{Phenological responses to warming}

We hypothesized that $C$. vulgaris would exhibit a stronger flowering phenology response to manipulative warming than $D$. subulatum. The date of first flowering of $D$. subulatum did not change in response to experimental warming $\left(2016: t_{(17.31)}=1.74, p=0.10 ; 2017: t_{(25.33)}=0.12\right.$, $p=0.90)$. However, $D$. subulatum achieved peak floral abundances 11 days earlier under 
warming $\left(t_{(19.12)}=2.69, p=0.01\right)$. In contrast, $C$. vulgaris responded to experimental warming by first flowering 18 and 11 days earlier in the 2016 and 2017 seasons, respectively (2016: $t$ (8.48) $\left.=5.07, \mathrm{p}<0.001 ; 2017: \mathrm{t}_{(9.94)}=2.63, \mathrm{p}=0.03\right)$. Peak flowering densities were also 11 days earlier $\left(t_{(11.91)}=2.133, p=0.05\right)$. We hypothesised that the differences in phenological responses between $C$. vulgaris and $D$. subulatum would result in a larger overlap of their flowering period. Under ambient temperatures, $2.79 \%$ of the total area under the curve for $D$. subulatum flowering occurred concurrently with high C. vulgaris floral densities (> 1000 flowers $\mathrm{m}^{-1}$ ). In experimentally warmed plots this overlap increased four-fold to $11.27 \%$ of total area under the curve (Figure 2.1A) Across the plant community, we observed no change in the flowering phenology of many native plant species under experimental warming with the timing of first flowering in 2017 and 2016 (Supplementary Figure 3 and Supplementary Figure 4, respectively) or in peak flowering (Supplementary Figure 5), largely unchanged. Consequently, there was no significant change in the date of first flowering by functional group in 2017 (forbs: $F_{(1,30)}=0.96, p=0.33 ;$ woody shrubs: $F_{(1,83)}=2.59, p=0.111 ;$ Supplementary Figure 6) or 2016 (forbs: $F_{(1,64)}=1.29, p=0.260$ and woody shrubs $F_{(1,105)}=3.02, p=0.08$; Supplementary Figure 7). However, woody shrubs achieved peak floral abundances earlier in the growing season ( $F_{(1,}$ $97)=14.003, p<0.001)$; this was not observed in forbs $\left(F_{(1,57)}=0.63, p=0.43\right.$; Supplementary Figure 8). 


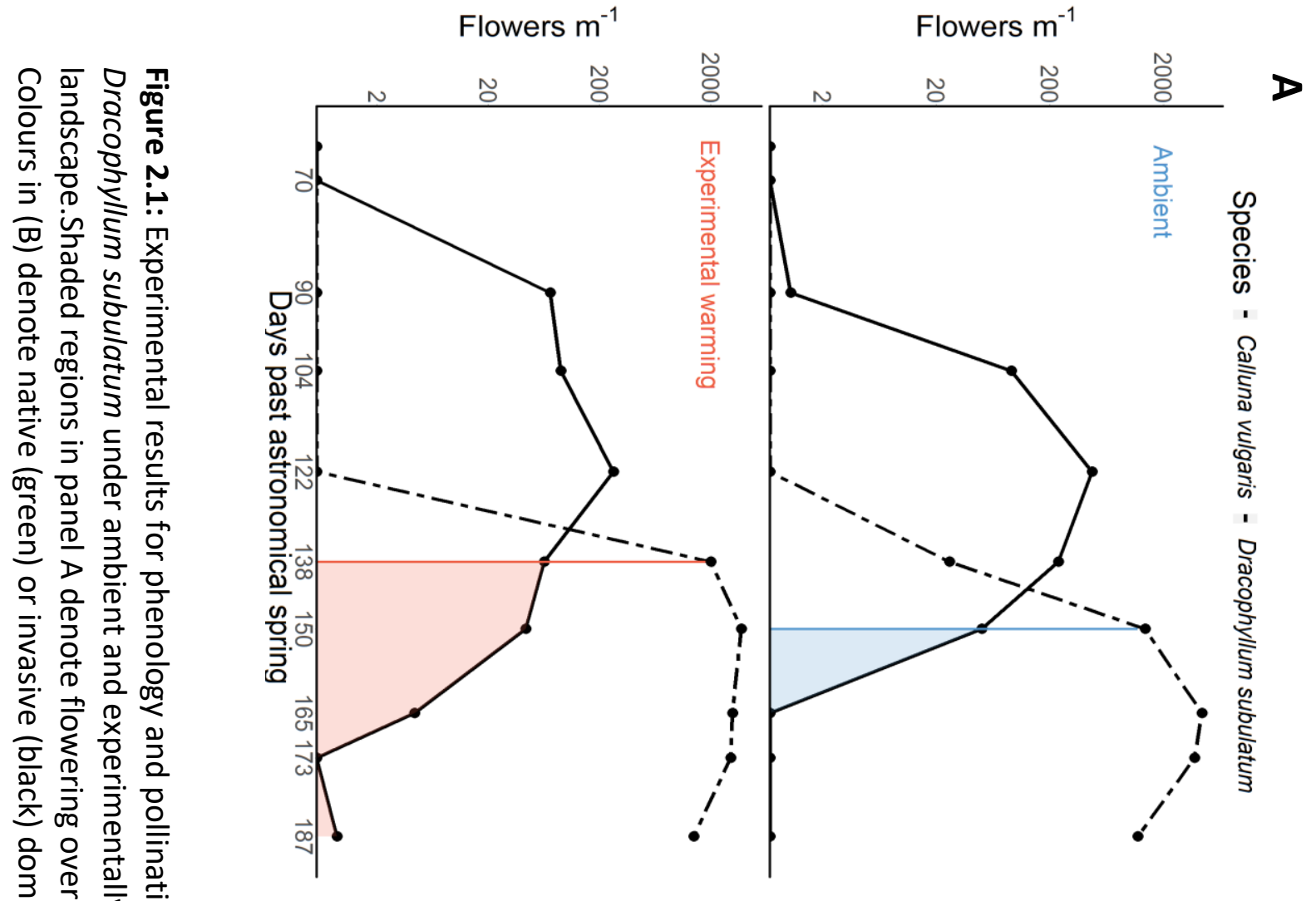$$
\text { \%) }
$$

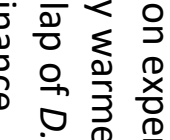

으 긍.

읠 을

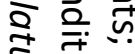

ई 은

思

于

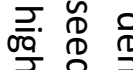

J

유 $\sum_{D} \frac{G}{\dot{X}}$

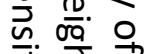

咅告

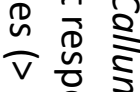

$\mapsto$ 음

ㅇㅇㅇ 心

중

웃 흥

กํํำ

윰

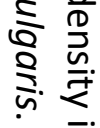

章

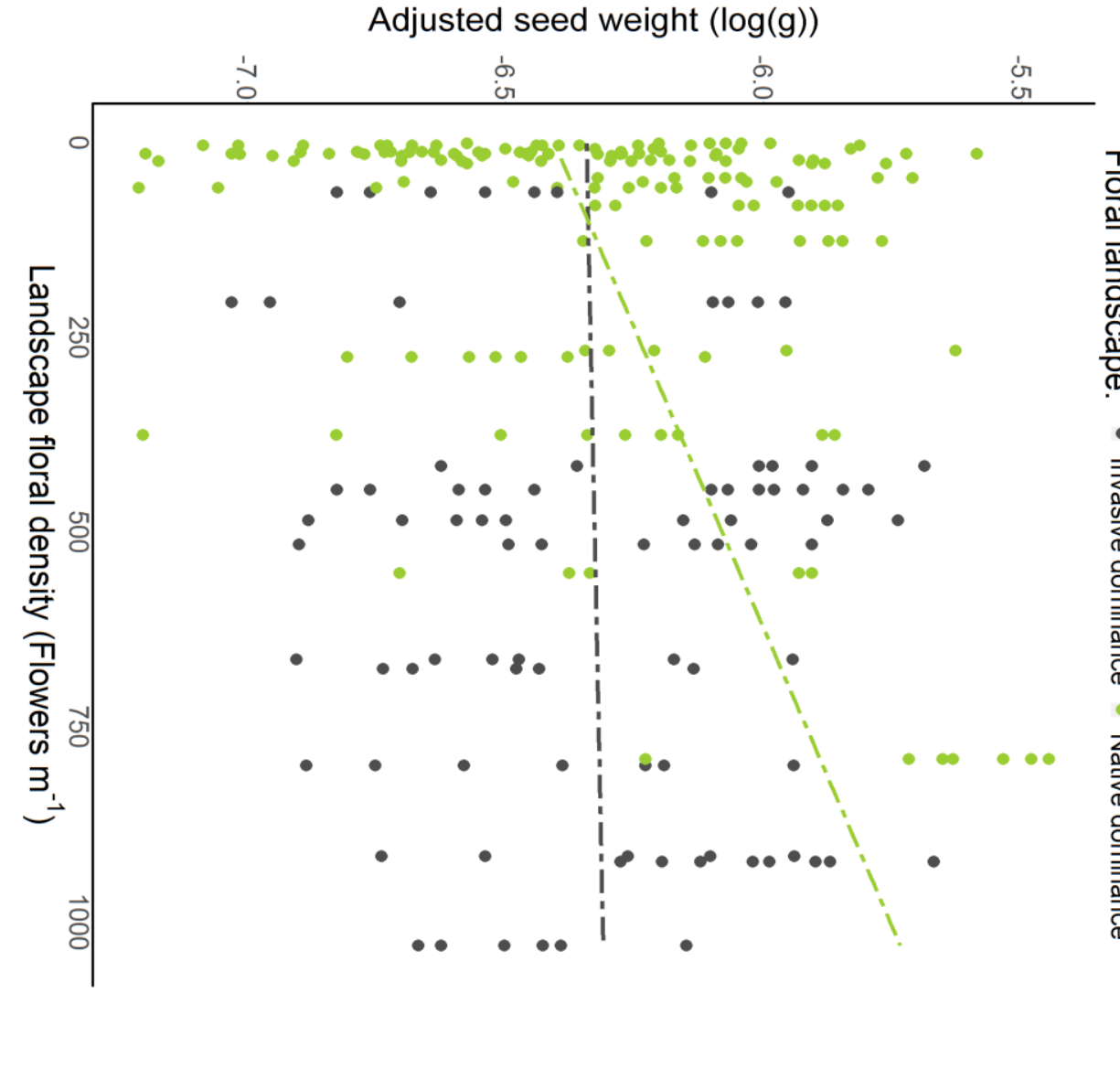




\section{Pollination landscape effects on seed weight}

To test our second hypothesis, that high densities of invasive flowers would have negative effects on $D$. subulatum reproduction, particularly in rich floral landscapes, we firstly accounted for the effects of other factors. Namely: 1-conspecific pollen donor availability, 2non-animal mediated pollination and 3- plant morphology. In our experiment, $D$. subulatum was unlikely to lack a conspecific pollen donor, as evidenced by the presence of conspecific in flower within a $5 \mathrm{~m}$ radius of $90 \%$, and a $10 \mathrm{~m}$ radius of $100 \%$ of $D$. subulatum plants in the 'natural pollination' treatment. The average floral density of $D$. subulatum within our landscapes was 18.0 flowers $\mathrm{m}^{-1}$, with a maximum floral density of $122.4 \mathrm{~m}^{-1}$ and a minimum recorded floral density of $0.4 \mathrm{~m}^{-1}$. We found significant differences in seed weight among pollination treatments, indicating that animal pollinator access to flowers has a significant effect on the reproduction of $D$. subulatum $\left(F=39.34_{(712,2)}, p<0.001\right.$; Supplementary Figure 9). We confirmed significant differences between the "natural" and "pollinator exclusion" treatments $(p<0.001)$ as well as the "hand pollination" and "pollinator exclusion" treatments $(p<0.001)$, with consistently lower corrected $D$. subulatum seed weights in the pollinator exclusion treatment. There was no significant difference between the "hand pollination" and "natural pollination" treatments, suggesting no general pollen-limitation among all landscapes. Floral landscape significantly affected $D$. subulatum seed weight. While landscape floral density $(t=-0.451, p=0.653)$ and the ratio of native and invasive flowers within the landscape $(t=-$ $0.763, p=0.446$ ) did not significantly affect seed weight, the interaction between them was significant $(t=2.472, p=0.014$; Figure 2.1B). Seeds were heaviest in landscapes with high floral densities and a large proportion of native flowers. Conversely, landscapes with a larger proportion of invasive flowers relative to native flowers were associated with consistently lower seed weights, irrespective of landscape floral density. 


\section{Discussion}

When native and invasive species flower simultaneously, pollination of the native species should change, especially if the invasive species is a prolific flower producer. Using two complementary experiments, we explored how warming and plant invasion impact native plant phenology and reproduction across an alpine landscape. We found a strong shift to earlier first flowering and peak flower production in C. vulgaris in experimentally warmed plots relative to control plots after both two and three years of treatment. In comparison, we found a significant shift to earlier peak flowering in native woody shrubs, but no coherent response in the date of first flowering in experimental warming treatments relative to control plots. Our focal native species, $D$. subulatum exemplified the response of native woody species, shifting its peak flower production earlier in the growing season but showing no response in the timing of first flowering. Consequently, we observed a significant increase in the flowering overlap of $D$. subulatum and $C$. vulgaris. This suggests that invasion by $C$. vulgaris and climate warming has the potential to interact to affect the reproductive success of $D$. subulatum. To investigate the potential consequences of this increased flowering overlap, we quantified the effects of the pollination landscape on seed weight in D. subulatum. We found a significant effect of experimental pollinator exclusion, indicating that seed weight in D. subulatum declines with pollen limitation. In flower-depauperate landscapes, $D$. subulatum seed weight was unaffected by the presence of invasive $C$. vulgaris flowers. However, in rich floral landscapes, a higher proportion of $C$. vulgaris was associated with lower $D$. subulatum seed weight. Our results therefore agree with previous work showing that co-flowering species can both facilitate and compete for pollination services and that these effects depend on overall flower abundance (Ekroos et al. 2015).

The invasive species in this study was more phenologically plastic in response to warming than native species with the same growth form. This result aligns with previous work suggesting that $C$. vulgaris flowering phenology is plastic, and potentially temperature controlled, displaying high variability both in New Zealand and its native range (Chapman and Bannister 1995). Invasive species generally exhibit greater intraspecific phenological variation than native species (Gallinat et al. 2018). It is unclear whether this is a result of phenotypic 
plasticity or genetics (Rapson and Wilson 1992, Wolkovich and Cleland 2014). Asynchronous phenologies among sympatric flowering species may reflect niche-partitioning for interactions with animal pollinators in systems where pollination services to plants are scarce (Cummings et al. 2014). Consequently, invasive species may adapt to local conditions, minimising flowering overlap with natives. In doing so, these species could ameliorate pollinator limitation under longer growing seasons driven by climate change, thereby facilitating pollination to native species. This is particularly likely if an invasive species flowers early or late in the season relative to the recipient native community. Alternatively, if an invasive plant responds to climate change by altering its phenology in a manner that increases flowering overlap with the native plant community (as we observed), we would expect sexual reproduction of native plants to decline. Species that advance phenology with warming typically also increase in abundance and growth (Wolkovich and Cleland 2014), potentially enhancing facilitative or exacerbating competitive effects. The degree to which an invasive species modulates the pollination success of natives is therefore contingent on the plasticity of their flowering phenology. Here, we focused on the flowering overlap of native and invasive species. However, climate change may additionally impact pollen or nectar composition (Hoover et al. 2012, Byers 2017, Nickless et al. 2017). Moreover, we manipulated temperature, but not other environmental cues that affect plant phenology (Wolkovich et al. 2012, Byers 2017, Nickless et al. 2017). There remain many unexplored avenues for future research.

As hypothesised, the invasive plant $C$. vulgaris had a negative impact on native plant reproduction in landscapes with high floral densities, but not in florally depauperate landscapes. This is important, because pollen limitation may have negative long-term effects on the persistence of animal-pollinated plant species (Ekroos et al. 2015). Declines in sexual reproduction of native species may reflect their competitive exclusion from the mutualistic plant-pollinator network (Brown et al. 2002), or because the quality of the pollination service to native species is compromised as a result of the excessive transfer of heterospecific pollen (Miller et al. 2018). Native plant species that require pollen transfer by generalist pollinators, as in this case, may be most vulnerable to competition for pollination from an invasive plant (Mitchell et al. 2009). Our findings align with those of previous studies, showing that native 
reproduction is compromised if native flowers are strongly outnumbered by the invasive flowers (Chittka and Schurkens 2001, Mitchell et al. 2009). Invasive plants may also be stronger competitors for pollinators than natives because of their floral characteristics (Chittka and Schurkens 2001). Floral characteristics could be important in this study as the native community is dominated by small white flowers, while flowers of $C$. vulgaris are pink. In agreement with others, our results indicate that concurrent flowering of native and invasive species will not necessarily generate a pollinator deficit for native species (Chittka and Schurkens 2001, Schweiger et al. 2010, Yang and Rudolf 2010). Instead, we find that the effects of invasion on the pollination services delivered to native plants depend on floral resources of the landscape. For example, per capita pollinator visits may increase for all species as a result of the heightened attractiveness of the patch when an invader is present (Mitchell et al. 2009, Ekroos et al. 2015). Factors such as pollinator behaviour (Popic et al. 2013, Blaauw and Isaacs 2014), flower density (Mitchell et al. 2009), floral richness of patches (Blaauw and Isaacs 2014), distance to resources required by pollinators (Ekroos et al. 2015), local pollinator community structure (Jędrzejewska-Szmek and Zych 2013) and abiotic conditions within microhabitats (Primack 1983) all contribute to the pollination success of plants. The implications of these patterns for pollination success is not straightforward (Ekroos et al. 2015) and makes generalisations across systems difficult.

Pollen limitation is common among native outcrossing angiosperms (Potts et al. 2010). Understanding the constraints on pollination of native species is especially pertinent as pollinators are declining globally (Potts et al. 2010) leading to an associated loss of reproductive capacity in plants (Cummings et al. 2014). D. subulatum reproduces both sexually and asexually; nevertheless, the maintenance of sexual reproduction is important for the long-term persistence of species with mixed reproductive strategies (Weppler et al. 2006). Our results illustrate novel mechanisms through which climate change and invasion may interact synergistically to reduce pollination services to native plants. In this ecosystem, we do not find that invasive plants will ameliorate climate change impacts on pollination services by providing resource stability, which is one potential non-linear outcome of interacting global environmental change drivers (Schweiger et al. 2010). Rather, we find increasing potential for 
competition for pollination. Further research is required to determine the species and landscapes most affected by this synergism, as well as other generalities in this field (Charlebois and Sargent 2017). Factorial experiments to partition the interactive effects of climate change and biological invasions on competition for pollination are lacking, as are combinations of observational and experimental designs (González-Varo et al. 2013, Byers 2017). Creative experimental approaches over a variety of spatial and temporal scales are required to elucidate the effects of global change drivers on ecosystem function (Fox et al. 2014, Byers 2017). Our study illustrates the utility of combining experimental approaches to investigate interactions among drivers of global change on the reproduction of native plant species. 
3 Elevational gradients alter invasion effects on species richness at multiple spatial scales

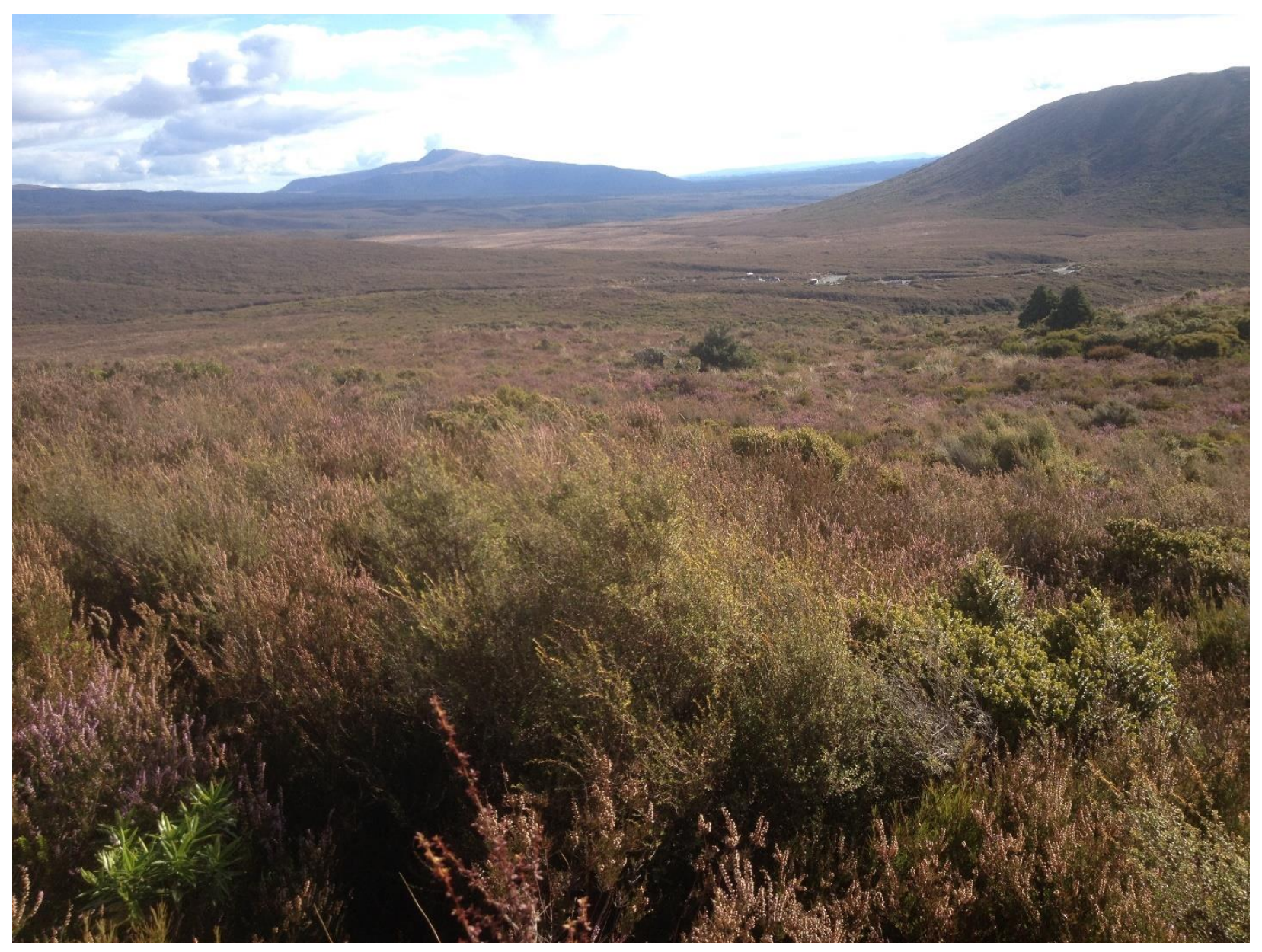

A native plant community is invaded by Calluna vulgaris near Mangatepopo carpark.

Giejsztowt, 2018 


\section{Introduction}

The presence of invasive plant species often reduces the abundance and diversity of native plants (Nielsen et al. 2015, Fox 2018, Michelan et al. 2018) and may increase the risk of extinctions (He and Hubbell 2011), however, the effects of invasive species on native plant richness typically vary with the scale of investigation (Powell et al. 2013, Case et al. 2016, Socolar et al. 2016). It is expected that climate change may alter the impact of invasive species on ecosystems (Brooker 2006) by modifying competitive conditions between native and invasive species (Schweiger et al. 2010). For example, abiotic conditions modulate the competitive ability of individuals by favouring different plant traits (Brooker 2006, Buckley et al. 2010, Gilman et al. 2010, Wisz et al. 2013, Michalet et al. 2014). The sensitivity of invasive species effects on plant species richness to changing abiotic conditions across multiple spatial scales is unknown.

Large scale patterns of vegetation are typically driven by climate, whereas the consequences of species interactions generally play out locally (Nielsen et al. 2015, Rojas and Zedler 2015), although in some circumstances species interactions affect richness at larger scales (Callaway et al. 2002, Cavieres et al. 2014). Consequently, studies comparing the effects of global change drivers on species richness at different spatial scales may lead to inconsistent magnitude or even sign of effects (Chase and Knight 2013). The Species Area Relationship (SAR) quantifies species richness at multiple scales (Arrhenius 1921) and is often derived from nested sampling strategies such as Whittaker plots (Stohlgren and Rejmanek 2014). This relationship has two important constants. The intercept $-c$ - indicates species richness at the smallest scale investigated. The slope $-z-$ indicates the rate of species richness accumulation with scale. SAR is an important management tool because $z$ is a scaling parameter; $z$ can be used to predict changes in the regional species pool from changes in small scale assemblages and to elucidate mechanisms driving geographical patterns in species richness (Socolar et al. 2016).

Plant species invasions typically alter community richness in two contrasting ways: by increasing richness through the addition of new, non-native species, or by decreasing it through the competitive exclusion of natives (Socolar et al. 2016, Hillebrand et al. 2018). Curiously, 
invasive species typically outcompete natives locally, but rarely cause species extinctions at the landscape scale (Powell et al. 2013, Case et al. 2016, Socolar et al. 2016), a pattern termed the 'invasion paradox' (Gaertner et al. 2009). Powell et al. (2013) elegantly reconciled this paradox by showing that densely invaded communities are associated with reduced species richness at small scales but also a higher $z$ than uninvaded communities. The mechanism driving this pattern is chiefly neutral selection: the random exclusion of individual plants by invasives, irrespective of their species-specific traits, leads to the disproportionate decline of regionally common species while rarer species are relatively unaffected (Powell et al. 2013, Case et al. 2016). Thus, the effect of invasive species on native species at large scales is typically to reduce their density or frequency rather than exclude them (Wisz et al. 2013). The frequency of a native species in a landscape has important implications for its persistence (Sexton et al. 2009). However, the presence of a non-native species does not universally lead to impacts on plant communities (Alexander et al. 2016). Effects are commonly dependent on the density and identity of the invasive plant (Bradley 2013, Nielsen et al. 2015). For example, Rojas and Zedler (2015) found that high density invasion of Phalaris arundinacea reduced species richness in a sedge meadow by half irrespective of the spatial scale of investigation. Further, a meta-analysis indicated inconsistent relationships between invasion, local richness and $z$ when invader density is included (Stohlgren and Rejmanek 2014). The nature of invasive species effects on species richness should be contingent on the intensity of invasion. In fact, non-linear responses could be expected, as they are a prevalent feature of ecological systems (De'ath and Fabricius 2000), particularly to global change (Tylianakis et al. 2008). Consequently, incorporating the density of invasive species can reveal critical thresholds at which invasion has important implications for SAR.

Environmental conditions can affect SAR indirectly by altering interactions among species. Alpine ecosystems are frequently used by ecologists to infer community responses to environmental stress because temperature decreases systematically with elevation over small geographic scales (Körner 2007, Alexander et al. 2016). The high alpine zone is considered to be an abiotically stressful environment in mesic mountain regions (Michalet et al. 2014). Species richness also varies as a direct result of elevation (Grytnes 2003, Bhatta et al. 2018), although it 
remains unclear how altitude-driven patterns of species richness play out at different spatial scales (Qiao et al. 2012, Bhatta et al. 2018). The most commonly reported species richness pattern in alpine ecosystems is unimodal, with peak richness at intermediate altitudes (Sanders and Rahbek 2012, Wang et al. 2018). This may be explained by environmental factors (Wang et al. 2018) including energetics (Storch et al. 2005, Qiao et al. 2012), however it can also be the consequence of null processes such as the geometry of species' ranges in bounded systems (Mid Domain Effect; Colwell et al. 2004), available area (Vetaas and Grytnes 2002, Xu et al. 2017, Bhatta et al. 2018, Hillebrand et al. 2018) and Rapoport's rule (Zhang et al. 2018).

Species richness may also vary along elevational gradients because of the changing nature of species interactions. The Stress Gradient Hypothesis (SGH) describes a switch in the sign of net species interactions along stress gradients (Choler et al. 2001, Callaway et al. 2002, Brooker 2006, Dvorsky et al. 2013, He et al. 2013). Generally, competition should dominate under benign environmental conditions and facilitation under stressful conditions. Facilitators can increase the diversity of other species by ameliorating factors such as temperature, wind abrasion, unstable substrate and solifluction, and low nutrient and water availability in stressful environments (Dvorsky et al. 2013). Plant traits, which vary with elevation, may be the mechanism by which species interactions change sign along stress gradients. For example, the facilitative effects of cushion plants are inversely correlated with productivity (Cavieres et al. 2014), and the size of trees determines whether they have net facilitative or competitive effects on alpine species at the treeline (Malanson and Resler 2016). Native-invasive plant interactions may also vary in magnitude and sign with environmental stress. For example, He et al. (2013) found that the competitive effects of an invader on species richness were less pronounced at high elevations. In some cases, facilitative interactions with invasive plants are recorded (Caceres et al. 2015).

Facilitative effects can also play a role in maintaining richness at larger scales (Callaway et al. 2002, Cavieres et al. 2014), however a pattern of increasingly facilitative interactions with environmental stress is not universal at larger spatial scales (Cavieres et al. 2014, Kopp and Cleland 2018). The effect of facilitative interactions may diminish with scale because environmental heterogeneity is an important driver of species richness (Bhatta et al. 2018). 
Nurse plants stabilise local abiotic conditions (Caceres et al. 2015) and may therefore homogenise patchy environments if they are widespread. For example, Caceres et al. (2015) showed that a nurse shrub species did not raise site-wide species richness despite a higher average species richness within nurse plants than outside them. This was attributed to greater variability in species identity in open habitats. Therefore, despite indications that interactions between invasive species and native communities are likely to change sign from competitive to facilitative with environmental stress, it is unclear how environmental stress may alter the effect of invasive species on SAR. We know of no study that investigates how the impact of invasive species on SAR is modulated by environmental stress.

We investigated the impact of invasion intensity on native alpine plant species richness at two spatial scales, and on the slope between them $(z)$, in 14 Whitaker plots positioned along gradients of elevation (3) and invasion intensity of Calluna vulgaris (L.) Hull in Tongariro National Park (TNP). We quantified native alpine plant species richness and measured invasion intensity through two metrics - canopy cover and aboveground biomass - in $1 \mathrm{~m}^{2}$ and $5 \mathrm{~m}^{2}$ quadrats. We expected to find a unimodal relationship between elevation and species richness at both the $1 \mathrm{~m}^{2}$ scale and the $5 \mathrm{~m}^{2}$ scales, leading to no effect on $z$. In accordance with the SGH, interactions between an invasive plant and species richness at small scales should become increasingly facilitative along environmental stress gradients. We therefore hypothesized (Hyp $\left.\mathbf{H}_{\mathbf{1}}\right)$ that the magnitude of both positive and negative effects of invasion on plant richness at the $1 \mathrm{~m}^{2}$ scale would increase with invader biomass, with the exception of positive interactions at very high invader biomass (Figure 3.1A).

Invasive species typically decrease local species richness through competitive interactions. However, they have a lesser effect on richness at larger spatial scales because local competition results in neutral sampling of species; this results in a higher $z$ (Powell et al. 2013). We expected to find this trend in environments with low abiotic stress, which is typical of low elevations in mesic alpine systems, where competitive native-invasive interactions ought to prevail in accordance with the SGH. In contrast, in high stress environments at high elevation where facilitative interactions among species should be more prevalent, species richness at larger spatial scales should be driven by habitat variability. Richness should be promoted by moderate 
canopy cover of the invader in abiotically stressful environments because some plant species require facilitative plant interactions to persist in high stress habitats, while others are stress adapted and are disproportionately found in open habitats. We therefore hypothesized $\left(\mathbf{H y p}_{\mathbf{2}}\right)$ that $z$ in low stress environments would increase monotonically with invader canopy cover, whereas in high stress environments the impact of invader canopy cover should be unimodal: moderate canopy cover should be associated with higher $z$ and contiguous canopy cover should be associated with a lower $z$ compared to uninvaded communities (Figure 3.1B).
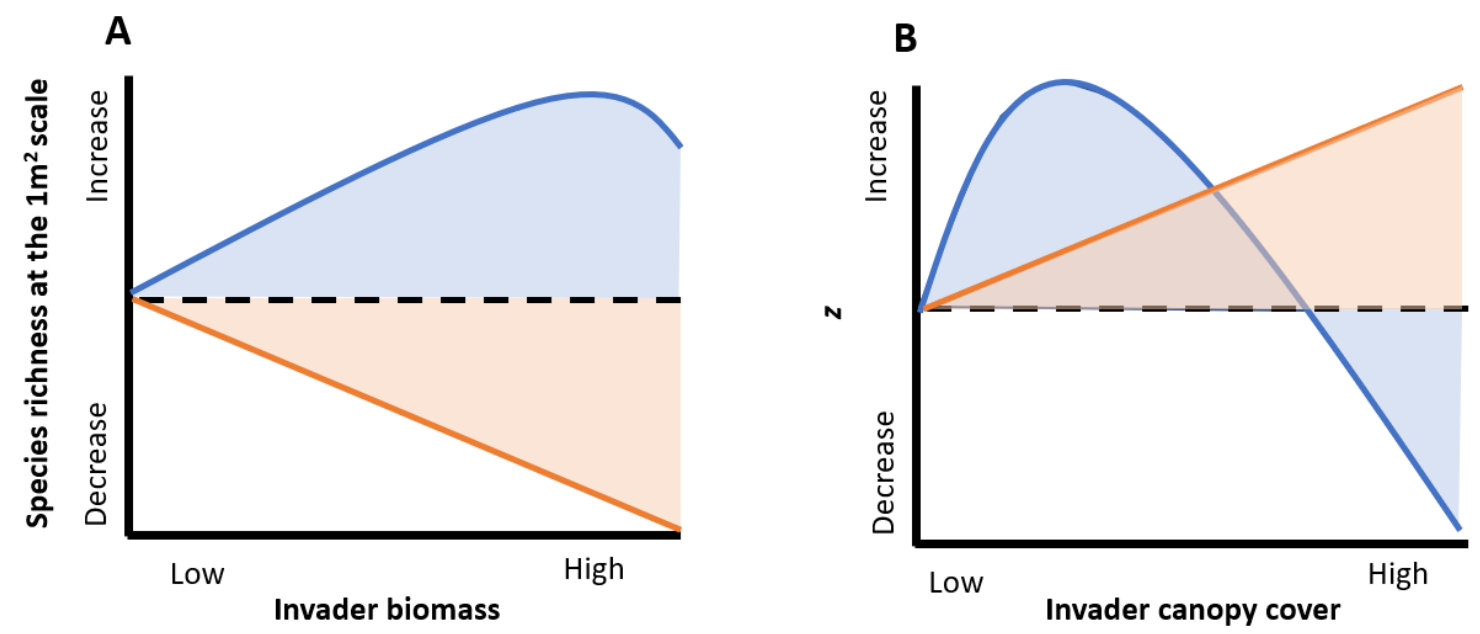

High environmental stress

Low environmental stress - - Expected value (uninvaded community)

Figure 3.1: Hypothesised response of plant species richness to invasion: $\mathbf{A}$ ) at the $1 \mathrm{~m}^{2}$ scale and B) on the slope of the Species Area Relationship (z). The effect of invasion should vary with environmental stress, with hypothesised effects at low environmental stress displayed in orange, and effects at high environmental stress in blue. 


\section{Methods}

\section{Community surveys}

\section{Study site}

We investigated species richness and $z$ along elevational and invasion gradients within Tongariro National Park (TNP), an alpine protected area in New Zealand $\left(-39.25^{\circ} \mathrm{N}, 175.58^{\circ} \mathrm{E}\right)$. The park is both a natural and cultural UNESCO World Heritage Site (Baird 2013) and hosts diverse alpine plant communities with high endemism (Chapman and Bannister 1990, Wardle 1991, Rogers and Leathwick 1996, Halloy and Mark 2003). The park encompasses three large volcanoes resulting in dark sandy-loam and loamy-sand soils (Chapman and Bannister 1990). Median annual air temperature on the lowlands of the central volcanic plateau is $9^{\circ} \mathrm{C}$ and decreases with elevation (Chappell 2015). C. vulgaris was intentionally introduced to the park in 1912 (Rogers and Leathwick 1996), occupying approximately one-third of the park by 1991 (Keesing, 1995) and continues to spread. C. vulgaris drives a decline of native and endemic plant species and affects insect assemblages in the park (Wardle 1991, Keesing 1995). In the park, C. vulgaris aboveground biomass is known to vary from 0.4 to $4.8 \mathrm{~kg} \mathrm{~m}^{-2}$ (Keesing, 1995), and biomass, canopy cover and height of $C$. vulgaris is related to altitude (Rogers and Leathwick 1996).

\section{Methodology}

Fourteen modified Whittaker plots (hereafter Whittaker plots) were established in TNP along three elevational gradients in the northwest, southwest and east of the park (Figure 3.2A). Plots were established at approximately every 100 vertical metres, with the lowest plots at the periphery of the park (970m a.s.I.), and the highest plots at the upper limit of the distribution of $C$. vulgaris (1530 m a.s.I.). The sites for plots were chosen to maintain a consistent hillside slope and aspect within transects. The layout of Whittaker plots emulates Powell et al. (2013; Figure 3.2B). This involves a nested sampling design, with ten $1 \mathrm{~m}^{2}$ quadrats and four $5 \mathrm{~m}^{2}$ quadrats nested within a $500 \mathrm{~m}^{2}$ plot. We cross-validated the area encompassed by each quadrat by measuring the length of all edges, as well as both diagonals. We allowed for a $5 \%$ error in all plot sizes. Most $1 \mathrm{~m}^{2}$ quadrats were placed systematically, but in some cases, they were shifted to ensure that plots with $C$. vulgaris are included. We recorded vascular plant 
species richness as well as $C$. vulgaris canopy cover (\%) in each quadrat and we included all cover which was rooted outside the plot.

C. vulgaris aboveground biomass is known to decrease with elevation in TNP (Rogers and Leathwick 1996). To assess changes in the aboveground biomass of $C$. vulgaris along the elevational gradients we estimated the canopy cover of $C$. vulgaris in $0.4 \mathrm{~m} \times 0.45 \mathrm{~m}$ quadrats in the vicinity of each Whittaker plot, then harvested the aboveground biomass. At each location, we took between four and five biomass quadrats $(n=59)$. Quadrats were taken to encompass as much variability in C. vulgaris canopy cover as possible, to build Whittaker plot site-specific relationships between $C$. vulgaris canopy cover and aboveground biomass. Harvested biomass was dried at $60^{\circ} \mathrm{C}$ to a constant weight and weighed. 


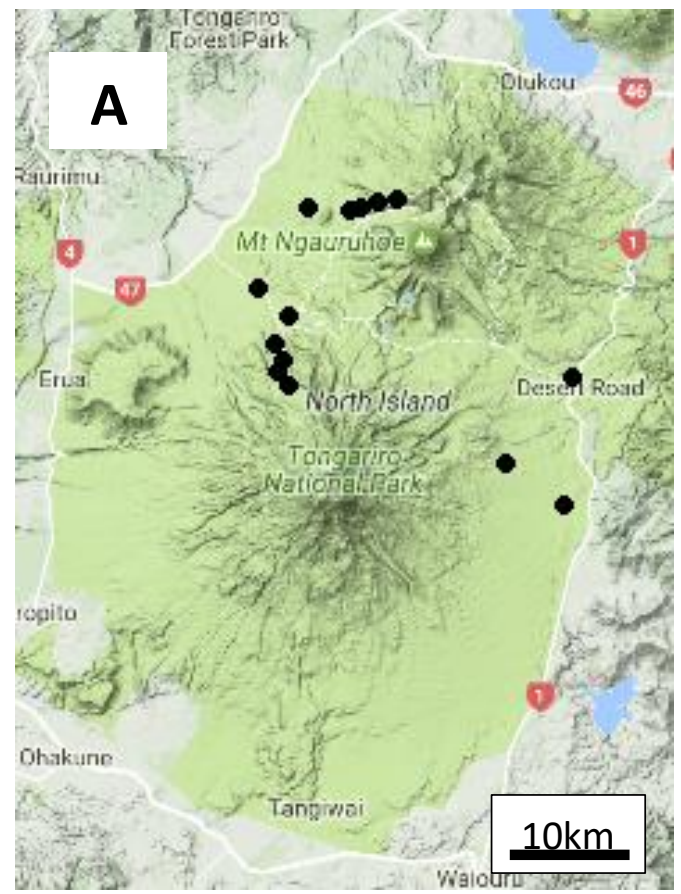

B

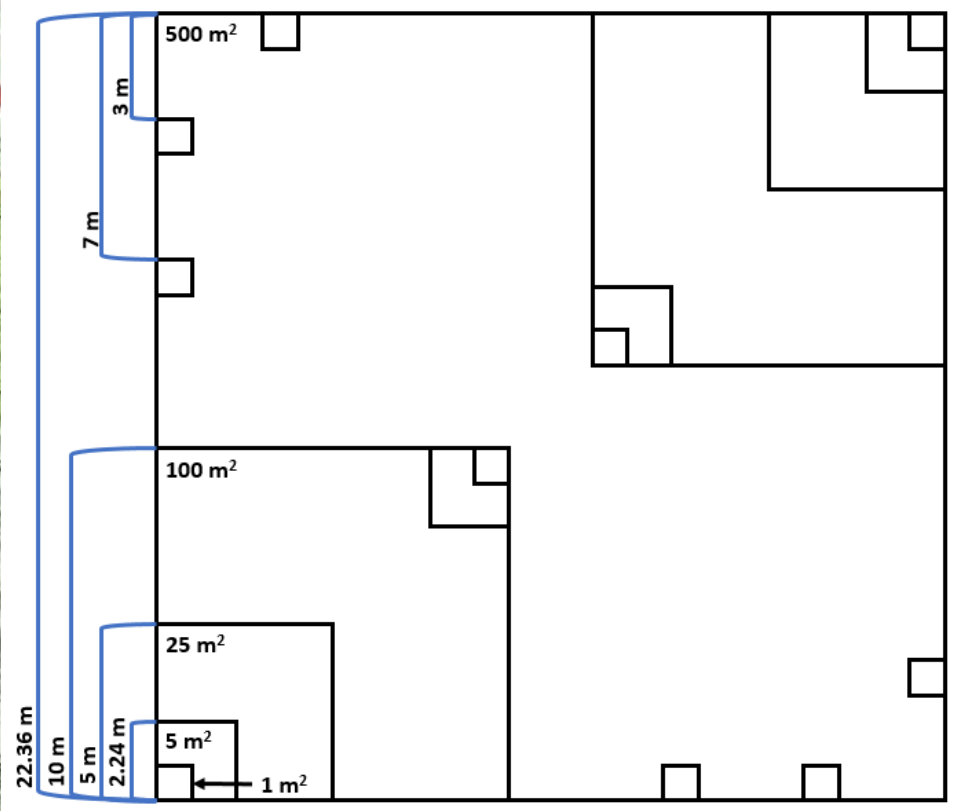

Transect Longitude Latitude

Mangatepopo

\begin{tabular}{c|rrr} 
& -39.143 & 175.586 & 1173 \\
\hline \multirow{4}{*}{ Rangipo } & -39.142 & 175.594 & 1269 \\
& -39.14 & 175.604 & 1377 \\
& -39.137 & 175.616 & 1452 \\
\hline \multirow{5}{*}{ Whakapapa } & -39.228 & 175.732 & 970 \\
& -39.293 & 175.727 & 1066 \\
& -39.272 & 175.688 & 1181 \\
& -39.182 & 175.527 & 1001 \\
& -39.197 & 175.547 & 1105 \\
& -39.21 & 175.538 & 1215 \\
& -39.22 & 175.543 & 1321 \\
& -39.225 & 175.539 & 1406 \\
& -39.233 & 175.547 & 1530
\end{tabular}

Figure 3.2: Species richness study sampling designA) Locations of Whittaker plots in Tongariro National Park, New Zealand are indicated on the map, coordinates and elevations are indicated in the table; B) Plot sampling design, modified from Powell et al. 2013.Quadrats range in size from $1 \mathrm{~m}^{2}$ to $500 \mathrm{~m}^{2}$ and follow a nested design. Only $1 \mathrm{~m}^{2}$ and $5 \mathrm{~m}^{2}$ scales were included in this analysis. Map (A) base layer was retrieved from maps.google.com. 


\section{Statistical analyses}

\section{Data preparation}

All analyses were performed in R version 3.3.1 (R Development Core Team, 2016). We included all native alpine vascular plant species (Mark 2012) in our measure of species richness. One quadrat was removed because the plot had no vascular plant species. We calculated a Whittaker plot site-specific relationship between $C$. vulgaris canopy cover and aboveground biomass based on the aboveground biomass removal data for each location. This was carried out using linear regression. The intercept was fixed at 0 for each linear model. Canopy cover significantly predicted biomass in all linear models (Supplementary Figure 10). The slope of these site-specific linear models was then used to predict $C$. vulgaris aboveground biomass from canopy cover for each quadrat in the analysis. Predicted C. vulgaris biomass (hereafter: 'biomass') for each quadrat was used in subsequent analyses as a predictor variable.

We calculated four $z$ values at each Whittaker plot location. $z$ values were estimated using the power law relationship, $S(A)=c A^{z}$ (Connor and McCoy 1979, Fattorini et al. 2017), where the species richness in the area $(A)$ is predicted by a log-log relationship with a slope of $z$ and an intercept of $c$ (Wilber et al. 2015). Species richness within nested quadrats was regressed against area using a log-log relationship with the ' $I \mathrm{~m}^{\prime}$ ' function in $\mathrm{R}$. We had a larger number of $1 \mathrm{~m}^{2}$ plots which were not nested within a $5 \mathrm{~m}^{2}$ plot, therefore dataset size in the following analyses was higher at the $1 m^{2}$ scale than at the $5 m^{2}$ scale or for $z\left(1 m^{2} n=139,5 m^{2}\right.$ and $z \mathrm{n}=55)$. We used only the 1 and $5 \mathrm{~m}^{2}$ scales in our analysis - this data subset allowed significantly higher statistical power in our models. Inclusion of the largest spatial scales would have allowed for only $n=14$, which would have limited ability to detect trends across scales. Some preliminary analyses of species-specific responses to invasion were carried out; these are not discussed here but are briefly summarised in Appendix 3.

\section{GAMs}

Next, we modelled the effect of $C$. vulgaris biomass and elevation on species richness at $1 \mathrm{~m}^{2}$, $5 \mathrm{~m}^{2}$ and $z$ using Generalized Additive Models (GAM) with the "gam" function in the package "mgcv" v. 1.8-26 (Wood 2018) as these are relatively robust to collinear predictor variables and we expected some collinearity between elevation and both measures of invasion intensity 
(Rogers and Leathwick 1996). Across the dataset, the predictor variables were tested for collinearity using Pearson's product-moment correlation with the function 'cor.test' and were all found to be within an acceptable range. The GAMs were fitted with the Restricted Maximum Likelihood (REML) smoothing parameter estimation method. The option 'select' was set to true so that redundant predictor terms in a model were penalized to 0 . For each of the three response variables, we calibrated nine GAMs of varying complexity, considering three predictor variables - elevation, $C$. vulgaris biomass, and C. vulgaris canopy cover - as well as their interactions (all model forms considered are provided in Supplementary Table 2). Models predicting $z$ were calibrated with $C$. vulgaris canopy cover and biomass values at the $5 \mathrm{~m}^{2}$ scale. The final models were selected based on Akaike Information Criterion (AIC; calculated using the 'logLik.gam' function in the 'mgcv' package; Wood 2011) and deviance explained ( $D^{2}$; derived from the summary of each model). Across the three modelled response variables (richness at a $1 \mathrm{~m}^{2}$ scale, richness at a $5 \mathrm{~m}^{2}$ scale and $z$ ), increasing model complexity was associated with higher $D^{2}$ and lower AIC (Supplementary Table 2). In each case, we selected the simplest model with the highest $D^{2}$.

\section{Standardized biomass}

Plant stature and productivity have a negative relationship with stress in mesic alpine environments and traits like size and density are associated with greater net competitive effects. To confirm that $C$. vulgaris plant size decreases with elevation, the dry aboveground biomass of $C$. vulgaris $(\mathrm{kg})$ harvested from each quadrat was divided by the canopy cover recorded at that quadrat to calculate 'standardized biomass' $\left(\mathrm{g} \mathrm{m}^{-2}\right)$. We applied linear regression using the 'Im' function to model the relationship between elevation and standardized biomass $(n=59)$. As the model did not conform to assumptions (BP = $8.0(1), \mathrm{p}=$ 0.005), the standardized biomass of $C$. vulgaris was Box-Cox transformed using the function 'BoxCoxTrans' in the package 'caret' v. 6.0-80 (Kuhn 2018). 


\section{Results}

\section{Model selection}

At a $1 \mathrm{~m}^{2}$ scale the interaction between elevation and $C$. vulgaris biomass was a particularly important source of deviance (Figure 3.3;Supplementary Table 2). Models including both $C$. vulgaris biomass and cover did not explain more deviance than those including only biomass as a predictor of species richness at this scale. The final model used to describe species richness at the $1 \mathrm{~m}^{2}$ scale included elevation, $C$. vulgaris biomass and an interaction between elevation and C. vulgaris biomass. The parametric coefficient was significant $(t=36.36 ; p<0.001)$ and $D^{2}$ was $38.6 \%$, indicating that the model fits provided significant reductions in deviance. The approximate significance of smooth terms was significant in all cases: elevation $(F=0.587, p=$ $0.005), C$. vulgaris biomass $(F=0.860, p<0.001)$ and the interaction between biomass and elevation $(F=1.380, p<0.001)$.

In contrast, at a $5 \mathrm{~m}^{2}$ scale, although the interaction between biomass and elevation was important in explaining variance, models including elevation, $C$. vulgaris biomass and cover explained substantially more deviance than any model which excluded one of these variables. The final model used to describe species richness at a $5 \mathrm{~m}^{2}$ scale included elevation, $C$. vulgaris biomass and an interaction between elevation and $C$. vulgaris biomass (as at $1 \mathrm{~m}^{2}$ ) but with the addition of $C$. vulgaris canopy cover as a predictor variable. The parametric coefficient was significant $(t=49.72 ; p<0.001)$ and $D^{2}$ was a mahusive $52.2 \%$. The approximate significance of smooth terms was significant for elevation $(F=0.88, p=0.002), C$. vulgaris $\%$ cover $(F=0.96, p$ $=0.002)$ and the interaction between biomass and elevation $(F=0.933, p=0.001)$. The direct effect of $C$. vulgaris biomass was not predictive.

Elevation, $C$. vulgaris biomass and cover were poor predictors of $z$ relative to the two richness models, and their interactions also explained less deviance. The interaction of elevation and $C$. vulgaris cover explained more deviance in $z$ than any other interaction. The final model used to describe $z$ included elevation, $C$. vulgaris canopy cover and the interaction between elevation and $C$. vulgaris canopy cover. This model was predictive but explained much less variation than those for $1 \mathrm{~m}^{2}$ and $5 \mathrm{~m}^{2}$ : the parametric coefficient was significant $(t=11.01 ; p$ 
$<0.001$ ) and $D^{2}$ was $16.7 \%$. The approximate significance of smooth terms indicated that only the interaction between $C$. vulgaris canopy cover and elevation was predictive for $z(F=0.533, p$ $=0.01$.

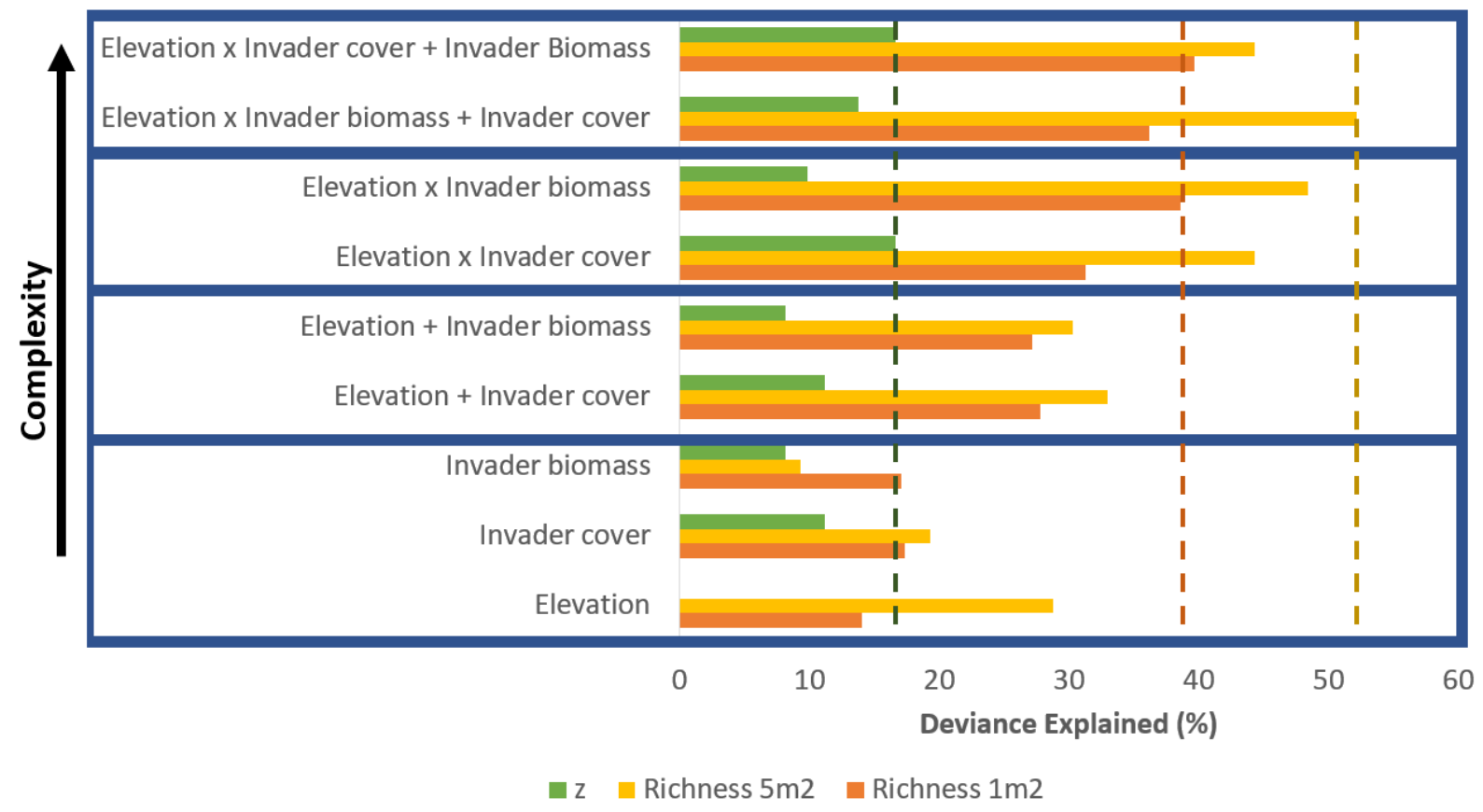

Figure 3.3: Generalized Additive Model (GAM) selection; a series of GAMs were generated to explain richness at $1 \mathrm{~m}^{2}$ and $5 \mathrm{~m}^{2}$ and for $z$ - the slope of the Species Area Relationship (SAR). Deviance explained by each model is graphed in order of decreasing complexity. Values are provided in Supplementary Table 2.

\section{Confirmation of expected trends}

We found support for our assumption that $C$. vulgaris standardized biomass declined with elevation $\left(F_{(1,57)}=44.79, p<0.001\right.$; Supplementary Figure 10). As expected (Supplementary Figure 11A), we found a unimodal relationship between plant species richness and elevation, at both $1 \mathrm{~m}^{2}$ (Supplementary Figure 11B) and $5 \mathrm{~m}^{2}$ scales (Supplementary Figure 11C), and no relationship between elevation and $z$ (Supplementary Figure 11D). We predicted that the relationship between $C$. vulgaris invasion intensity and species richness both at the 1 $\mathrm{m}^{2}$ and the $5 \mathrm{~m}^{2}$ scales was unimodal, therefore $z$ would be consistent across all elevations 
(Supplementary Figure 12A). As expected, at the $1 \mathrm{~m}^{2}$ and $5 \mathrm{~m}^{2}$ scale the relationship was unimodal; maximum species richness is associated with a $C$. vulgaris biomass of $\sim 2000 \mathrm{~g} \mathrm{~m}^{-2}$ (Supplementary Figure 12B), while at the $5 \mathrm{~m}^{2}$ scale, maximum species richness occurred at $\sim 50 \%$ C. vulgaris cover (Supplementary Figure 12C). In line with predictions, C. vulgaris cover had no significant direct effect on $z$ (Supplementary Figure 12D).

\section{Interactive trends}

The interaction of $C$. vulgaris invasion intensity and elevation were significant in all three GAMs. GAM predictions for species richness at a $1 \mathrm{~m}^{2}$ scale at three example elevations, $1072 \mathrm{~m}$, $1296 \mathrm{~m}$ and $1452 \mathrm{~m}$ - which are representative of low, intermediate and high elevations in TNP are provided in Figure 3.4. At the intermediate and high elevations, the relationship is unimodal, with peak species richness predicted at 3000 and $2500 \mathrm{~g} \mathrm{~m}^{-2} \mathrm{C}$. vulgaris biomass at the example elevations of $1452 \mathrm{~m}$ and $1296 \mathrm{~m}$, respectively. Conversely, at $1072 \mathrm{~m}$ increasing $C$. vulgaris biomass is associated with a monotonic decline in species richness. At a $5 \mathrm{~m}^{2}$ scale, richness at low elevation declines with moderate $C$. vulgaris canopy cover and then increases modestly (Figure 3.5). Conversely, at the intermediate and high elevations, the relationship is unimodal, with peak species richness predicted at 750 and $1000 \mathrm{~g} \mathrm{~m}^{-2} \mathrm{C}$. vulgaris biomass at the example elevations of $1452 \mathrm{~m}$ and $1296 \mathrm{~m}$, respectively. At the low elevation, the relationship is weak, with marginally higher richness at very low $C$. vulgaris biomass $\left(<500 \mathrm{~g} \mathrm{~m}^{-2}\right)$. The relationship between $z$ and $C$. vulgaris cover changed sign with elevation (Figure 3.6). At the high elevation, the relationship was unimodal, with $z$ peaking at 30\% C. vulgaris canopy cover and declining sharply at higher $C$. vulgaris covers. At the intermediate elevation, this relationship is maintained but is weaker. In contrast, at the low elevation, the relationship is also unimodal but inverted, with minimum $z$ associated with $30 \% \mathrm{C}$. vulgaris cover. 


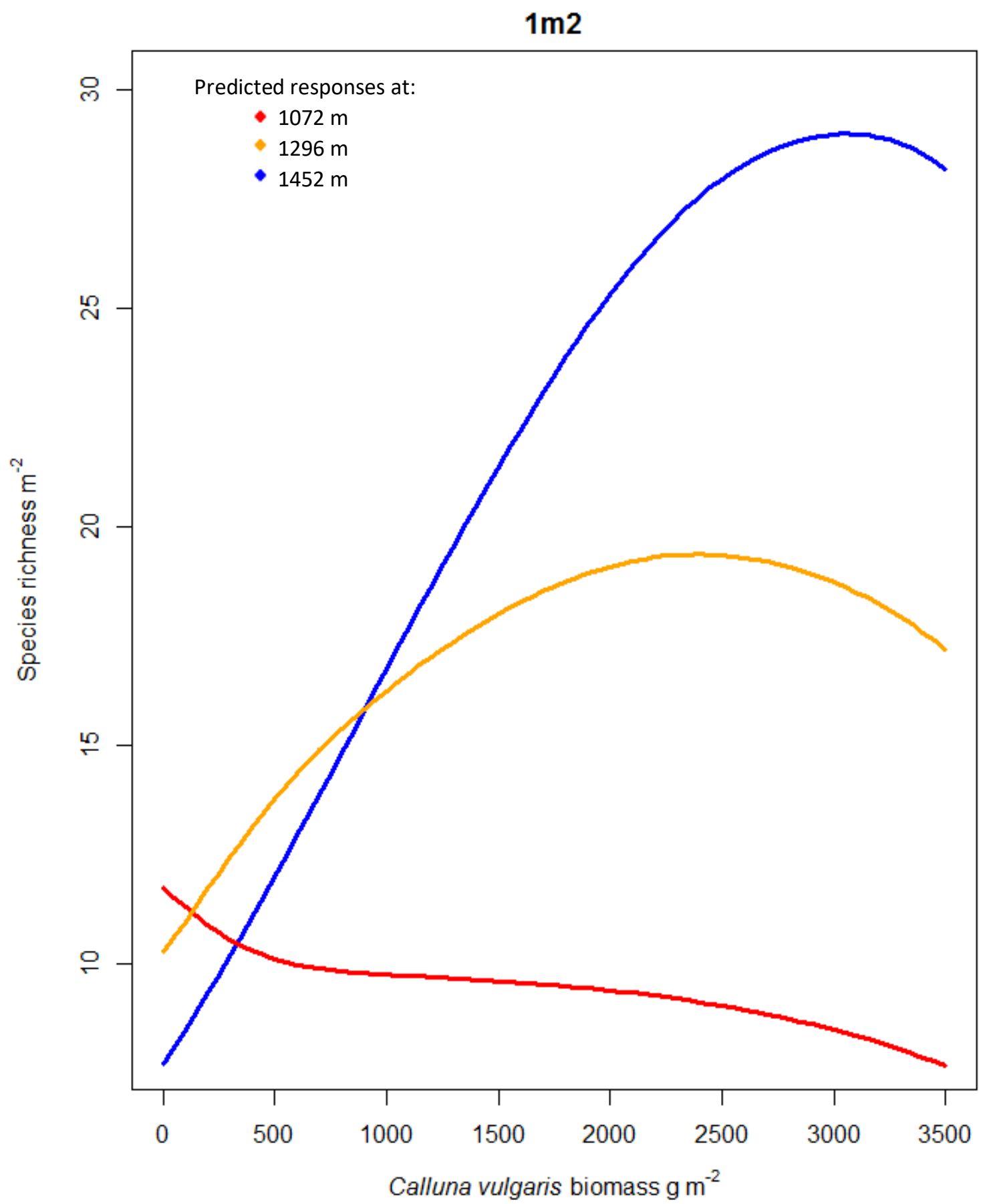

Figure 3.4: Modelled native alpine plant species richness at a $1 \mathrm{~m}^{2}$ scale as a function of Calluna vulgaris biomass $\left(\mathrm{g} \mathrm{m}^{-2}\right.$ ) in Tongariro National Park, New Zealand.Predictions are provided separately for three example elevations: $1072 \mathrm{~m}$ (red, representative of low elevation), 1296m (yellow, intermediate elevation) and 1452m (blue, high elevation). 


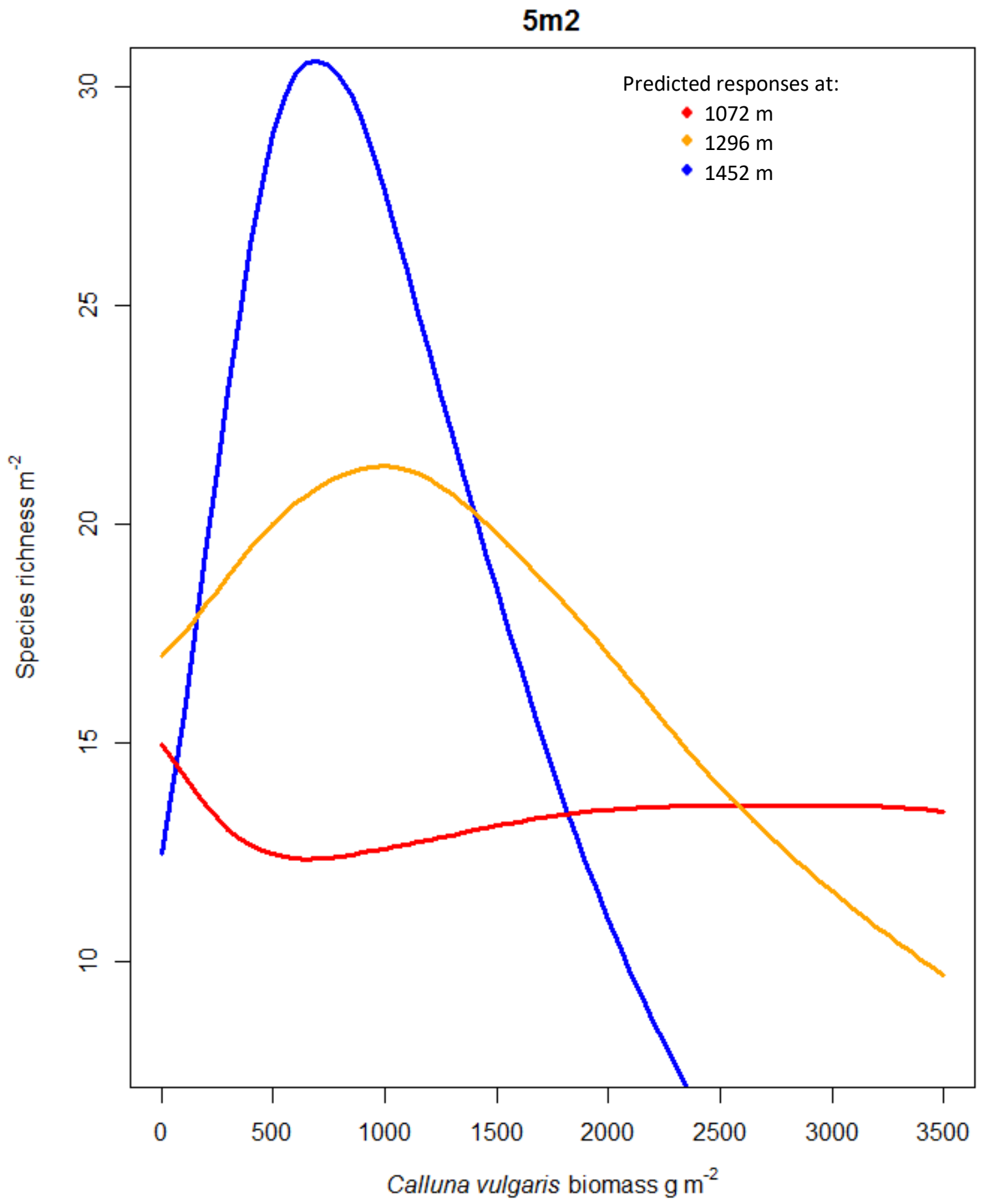

Figure 3.5: Modelled native alpine plant species richness at a $5 \mathrm{~m}^{2}$ scale as a function of Calluna vulgaris biomass $\left(\mathrm{g} \mathrm{m}^{-2}\right)$ in Tongariro National Park, New Zealand. Predictions are provided separately for three example elevations: $1072 \mathrm{~m}$ (red, representative of low elevation), $1296 \mathrm{~m}$ (yellow, intermediate elevation) and $1452 \mathrm{~m}$ (blue, high elevation). 


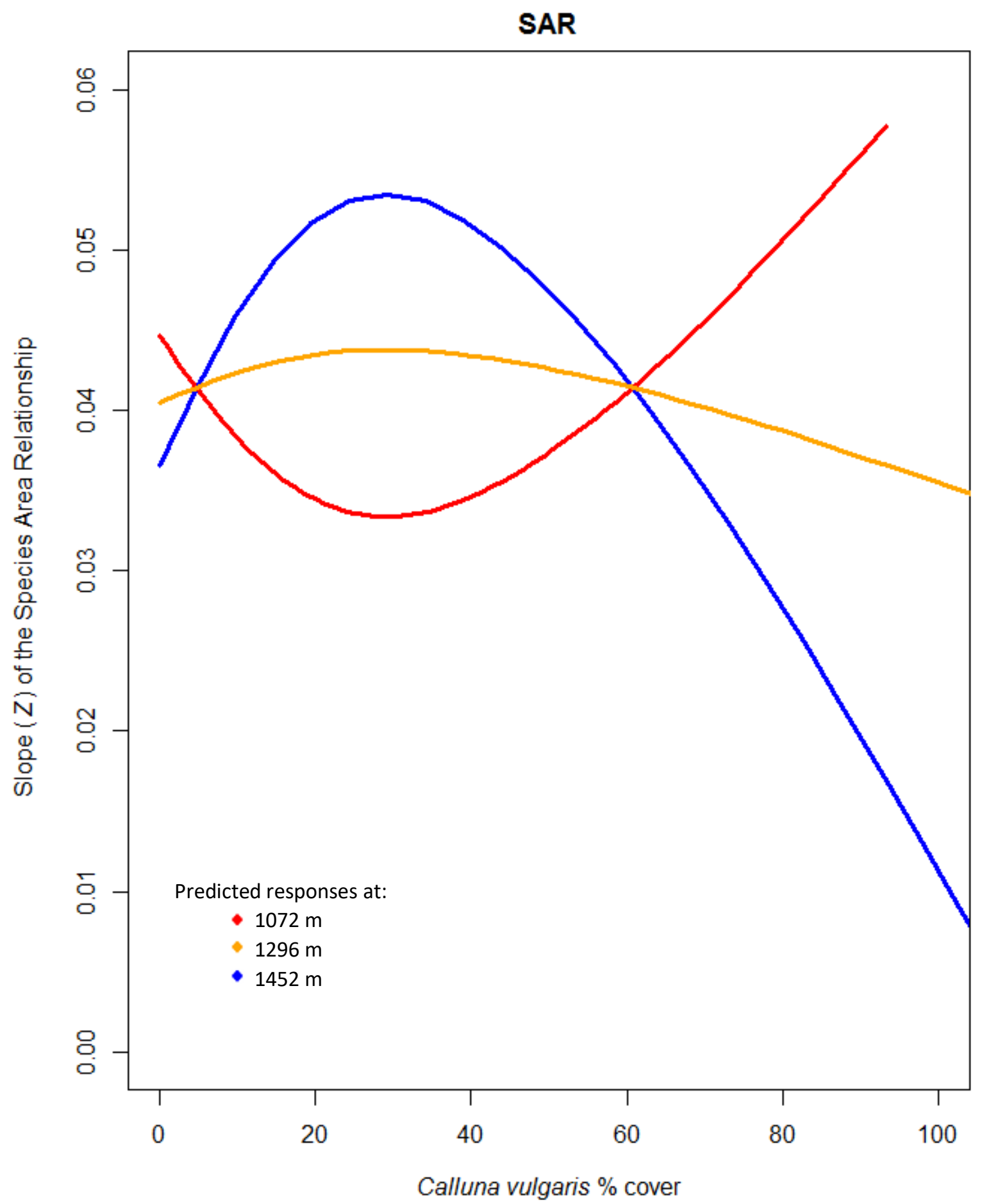

Figure 3.6: Modelled slope of the Species Area Relationship (z) for native alpine plant species at a $1-5 m^{2}$ scale as a function of Calluna vulgaris canopy cover in Tongariro National Park, New Zealand. Predictions are provided separately for three example elevations: $1072 \mathrm{~m}$ (red, representative of low elevation), 1296m (yellow, intermediate elevation) and 1452m (blue, high elevation) 


\section{Discussion}

Species invasions typically reduce species richness at small but not large scales (Powell et al. 2013), however this effect should be modulated by environmental stress conditions as well as by the intensity of the invasion. Here, we explored the correlation between invasion intensity and species richness at the $1 \mathrm{~m}^{2}$ and the $5 \mathrm{~m}^{2}$ scale, as well as the slope of SAR - $z$ along alpine elevational gradients. We used two measures of invasion intensity because we predicted that aboveground biomass of the invader would drive the strength of species interactions at small scales, whereas at larger spatial scales canopy cover would indicate the prevalence of invader impact. We found support for this prediction. We also found support for our expectation that the sign of the interaction between the invasive species and native species richness was dependent on environmental stress conditions (Callaway et al. 2002, He et al. 2013). Further, we found that, in line with $\mathbf{H y p}_{1}$, at small scales both positive and negative interactions were magnified by increasing invader biomass in most cases. Further, we found partial support for $\mathrm{Hyp}_{2}$, that the effect of invasive species canopy cover on $z$ in high stress but not low stress environments would be non-linear. Instead, we found that the effect of canopy cover on $z$ was unimodal in both cases. A key finding of our study is that the effect of invasive plants on both parameters of SAR can change in both magnitude and sign depending on environmental stress, indicating that species invasions may have variable consequences for native species richness at both small and large spatial scales. Furthermore, both invader biomass and canopy cover determine richness outcomes at larger spatial scales.

Global change drivers may have variable effects at different spatial scales (Chase and Knight 2013). Implicit in our hypotheses was the assertion that we expected different aspects of invasion to drive species richness responses at different scales. We anticipated that invasive species can affect SAR in two different ways: by facilitating or competing with neighbours, thereby changing $c$, or by augmenting or interacting with habitat heterogeneity, thereby changing $z$. Through our model selection process, we confirmed this assertion. Species richness at small scales was driven by invader biomass and therefore is likely a reflection of local scale interactions. In contrast, $z$ was driven by invader canopy cover across larger spatial scales, which should indicate the prevalence of local scale interactions. Canopy cover and biomass of 
the invader were was not strongly correlated, suggesting that these two aspects of invasion intensity can be relatively distinct. As a consequence of these two processes, richness at a $5 \mathrm{~m}^{2}$ scale was predicted by both measures of invasion intensity and our model explains a large proportion of deviance in species richness.

We found that the effect of an invasive species on native plant richness at the $1 \mathrm{~m}^{2}$ scale was dependent upon environmental stress conditions, which is in line with expectations

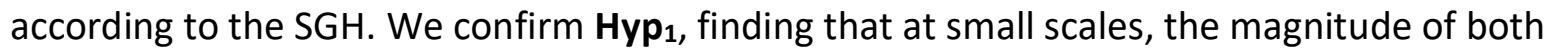
competitive and facilitative species interactions are contingent on invader biomass. Biomass linearly predicted competitive effects, which was expected because experimental data shows that larger removals are associated with greater biomass compensation (Rixen and Mulder 2009). This is likely to occur because traits such as stature and foliar density are reflected in biomass and are known to affect competition intensity and relative competitive ability (Carboni et al. 2018). However, in the case of facilitative interactions in high stress environments, we expected an inflection point above which the net interaction would be negative, because biomass shifts the balance of competition and facilitation (Malanson and Resler 2016). Our results show that at high elevation, the inflection point occurs at very high invader biomass. In this context it is important that we confirm, using standardized biomass, that high biomass $C$. vulgaris stands rarely occur at high elevations in TNP (Chapman and Bannister 1994, Rogers and Leathwick 1996). Therefore, at high elevation, native-invasive species interactions should be predominantly facilitative. Few studies report facilitative interactions between native richness and invasive species (Rodriguez 2006). Our results suggest that the potential for facilitative native-invasive interactions may considerable if invaders are morphologically plastic. If small plant stature results in net facilitative effects (Malanson and Resler 2016) and small plant stature is favoured by stressful high alpine environments (Körner 2013) then plastic invasive species should attain smaller stature at high elevation and have predominantly facilitative interactions.

The first aspect of $\mathrm{Hyp}_{2}$ was that the effect of the invasive species on the scaling parameter - z- can change sign with environmental stress. We find support for this hypothesis. This is expected in line with the SGH, as the nature of local interactions should change. 
However, this is a novel insight, because the prevailing literature suggests that invasive species effects should have consistent effects on $z$. We find partial support for the latter aspect of $\mathbf{H y p}_{\mathbf{2}}$; instead of a monotonic relationship at low elevation and a unimodal relationship at high elevation, we find a unimodal relationship with $z$ in both contexts. We expected to find a unimodal relationship at high elevation because the effect of nurse plants is to increase local diversity by facilitating some native species, however, where total vegetative cover is high over larger scales, we expect habitat homogenization through which open habitats that host high alpine specialists are lost. Abiotic conditions inside and outside the influence of a nurse species are typically substantially different (Caceres et al. 2015) and habitat variability drives the high diversity of high alpine systems (Caceres et al. 2015, Bhatta et al. 2018). Therefore, the greatest facilitative effect of nurse plants on species richness at large scales should occur where they are patchy, allowing for both nurse and open habitat types. Where nurse plants introduce a novel environmental condition, they may increase species richness at larger spatial scales (Callaway et al. 2002). Conversely, where invaders have high canopy cover, studies find that species richness at large spatial scales decrease (Rojas and Zedler 2015). Our results support this interpretation by showing that moderate canopy cover over large spatial extents promotes native richness.

In $\mathrm{Hyp}_{2}$ we predicted that in low stress environments, competitive effects should result in a monotonically increasing $z$. This hypothesis was based on previous work which demonstrated that invasive species outcompete species randomly (Powell et al. 2013). The resulting neutral sampling of species should lead to disproportionately large competitive effects for abundant species while rare species remain relatively unaffected (Powell et al. 2013). Contrary to this expectation, we found a unimodal relationship between invader canopy cover and $z$ in low stress environments; at low canopy cover, the effect of invasion was to decrease $z$. A decreasing $z$ indicates that competition with the invader did not result in the neutral sampling of species. Non-neutral exclusion of species may occur at low C. vulgaris canopy cover because the establishment of $C$. vulgaris is non-random. Observational studies suggest that the species establishes well in inter-tussock spaces, leading to the selective extirpation of prostrate intertussock shrubs and herbs (Chapman and Bannister, 1990). A more thorough investigation of 
species which may be particularly at risk of competitive exclusion is warranted. Some analysis pertaining to species identity is provided in Appendix 3.

Commonly, invasive species impacts on native community richness are evaluated by contrasting uninvaded and densely invaded communities (Stohlgren and Rejmanek 2014). However, the effect of invasive species is commonly driven by density (Bradley 2013, Michelan et al. 2018), and this is supported by the analysis presented here. We find that the consequences of invasive species on SAR are non-linear in response to both biomass and canopy cover. Further, the impact of invasion may be contingent on the identity of the invader (Traveset et al. 2008). Invasive plant species can produce local species interactions of different intensities because they vary in plant traits (Low et al. 2015). Further, the effect of local interactions on species richness at larger scales is dependent on spatial patterns of invasion which are also dependent on several variables (Shields et al. 2014). For example, soil characteristics can drive spatial patterns of species establishment, but have not been explicitly included here. In order to fully understand the implications of invasion for species richness, it is imperative that we incorporate such aspects of invasions. Furthermore, global change drivers are especially likely to elicit non-linear responses (De'ath and Fabricius 2000) as species interactions are altered by climate change (Tylianakis et al. 2008). Our results indicate that invasive species impacts across scales are non-linear. We advocate the use of non-binary studies of the effects of invasive species.

In alpine communities, many studies report that facilitative interactions drive nonnegligible patterns in richness (Butterfield et al. 2013, Dvorsky et al. 2013, Cavieres et al. 2014, Kikvidze et al. 2015). We support previous work indicating that invasive species can be facilitators of native biodiversity (Rodriguez 2006) and show that particularly in communities with high environmental stress, this effect is equal to or greater in size than the competitive effects which shape patterns of biodiversity at lower elevations. Species invasions to high alpine areas are increasingly leading to homogenization of high alpine communities (Jurasinski and Kreyling 2007). This may occur because of the increasing preponderance of nurse facilitated species, and the exclusion of open habitat specialists. Consequently, facilitative interactions with invasives need further investigation to understand their broader impacts on species 
richness. Furthermore, habitat variability may be key parameter determining the sign of the impacts of facilitative interactions at larger scales.

A large limitation of this study is the use of vegetative surveys, which conflate correlation with causation. For example, it is important that the correlation between invasive species canopy cover and native plant richness at high elevation could operate through one of two mechanisms. Firstly, the invasive species could act as a nurse, ameliorating the abiotic conditions experienced by native species. In this case, native species interactions with the invader would drive species richness patterns. Alternatively, invasive canopy cover patterns could be driven by underlying habitat variability. For example, variability in substrate quality may drive invasive canopy cover patterns. In this case, invasive canopy cover would also correlate with richness patterns, provided that richness and invasive species cover respond similarly to habitat variability. The survey data presented here does not allow us to disentangle correlation and causation, and we suggest removal experiments are required to further investigate the mechanism behind these patterns (Hector and Loreau 2007, McLaren and Turkington 2011). A similar issue arises from interpretation of this data in the context of climate change. If plant interactions respond to climate change in a way that is analogous to their behaviour along stress gradients, then we predict that competitive interactions between the native community and invasive species are likely to prevail under climate change. However, elevational gradients constitute a number of correlated abiotic gradients, including gradients of temperature, water availability, disturbance, and many other factors (Whittaker 1967), not all of which may be analogous to those imposed by future climate change. The relative contribution of environmental gradients and null effects to species richness patterns along elevational gradients are widely debated and appear variable among ecosystems (Zhang et al. 2018). Standardization of sampling effort may not be sufficient to overcome the contribution of null patterns, allowing the comparison of communities (Chase and Knight 2013). Consequently, we cannot predict climate change impacts on SAR in our study system. However, a description of pattern may remain useful in anticipating the direction of climate change predictions. A recent study comparing the importance of factors for predicting SAR along an elevational gradient concluded that climatic variables were the most important predictive factor and that it 
was reasonable to expect that global warming may result in an uphill shift of peak richness (Zhang et al. 2018).

An additional limitation of our approach is the use of richness as a response variable. Biodiversity is multifaceted, including richness, but also species identity, dominance and rarity (Hillebrand et al. 2018). Richness is an informative metric as it is associated with community stability and ecosystem functioning (Hillebrand et al. 2018, Wang et al. 2018). SAR provides more information than simple richness measures because SAR indicates the size of the geographic area which is required to maintain species richness (Socolar et al. 2016). However, invasive species may affect dominants and subordinates (Rojas and Zedler 2015) or various life forms (Nielsen et al. 2015) differently. Consequently, species extinctions are non-random (Isaac and Williams 2013). Our data indicate that the competitive exclusion of species by the invader in our study system can be non-random. We cannot identify the species which are excluded in this analysis, but we provide some insight into the identity of vulnerable species in Appendix 3. There is no unified framework for understanding the effects of invasive species on biodiversity (Vila et al. 2011). To understand the full effect of invasive species on biodiversity, metrics which account for species identity should also be considered.

Global environmental change is likely to facilitate range expansion of invasive plants into alpine ecosystems through increasing propagule pressure, and a reduction of abiotic filtering (Alexander et al. 2016), thereby increasing the urgency in understanding the potential for plant invasions to alter biodiversity patterns. Our study illustrates that the impact of invasive species on SAR is highly dependent on environmental stress as well as both the biomass and prevalence of the invasive species. Our results suggest that the impact of invasive species on spatial diversity patterns may change with environmental stress. Further, we find that invasion intensity determines the outcome of interactions between invasive species and the native community. Therefore, predictions aimed at contributing to effective conservation management in landscapes affected by invasion in a changing climate require more than simple predictions of invader presence. 
4 Density-dependent effects of an invasive species on the spatial distribution and abundance of a native species under future climate scenarios

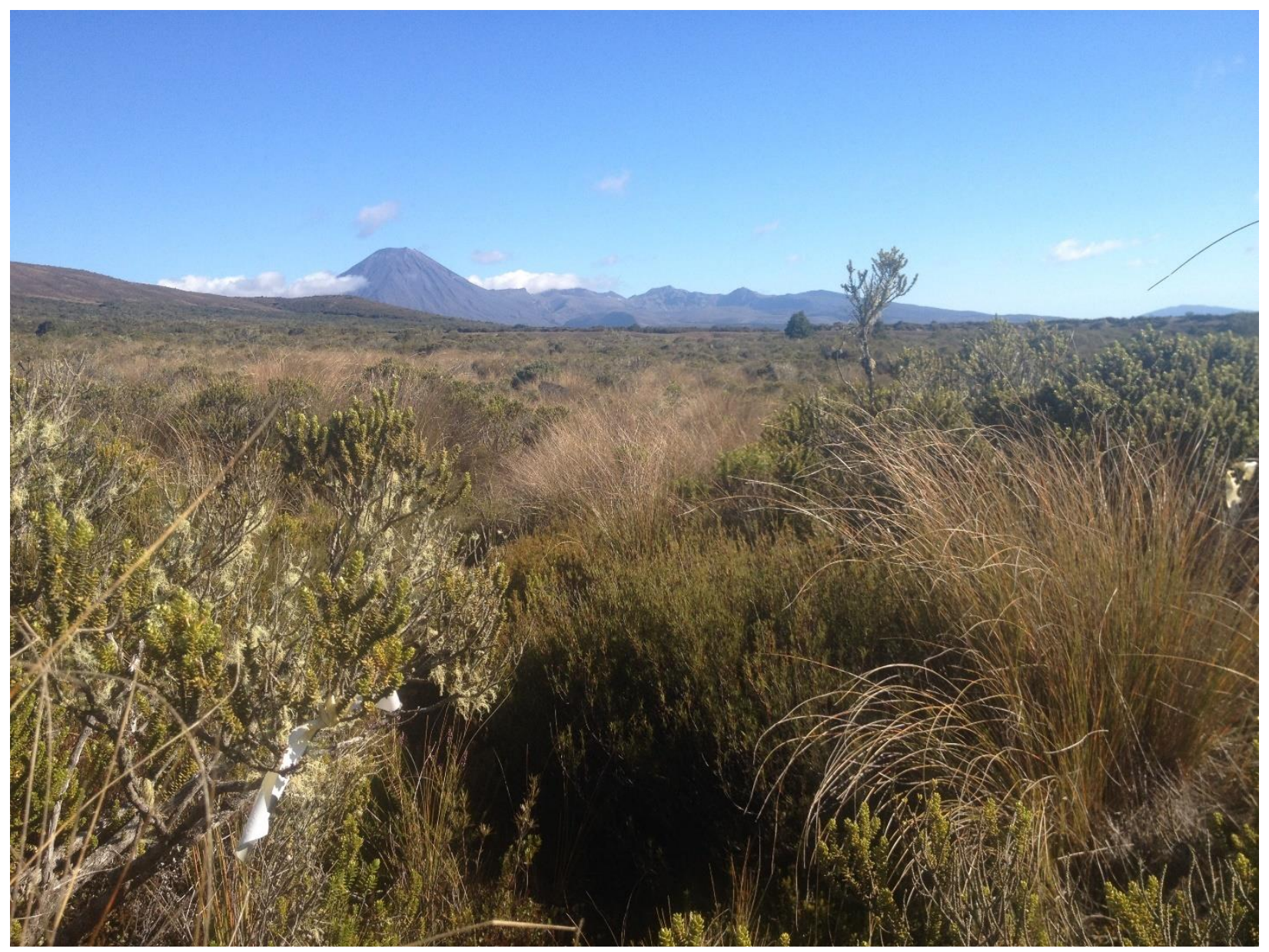

Chionochloa rubra (red tussock) in a community that is uninvaded by Calluna vulgaris in the Rangipo desert.

Giejsztowt, 2018 


\section{Introduction}

Climatic change is a direct cause of shifts in plant species' distributions (Parmesan and Yohe 2003), but may also have indirect effects on plant distributions by altering interactions between species (Wisz et al. 2013). Indirect effects of warming mediated through species interactions are predicted to be the greatest threat to biodiversity and ecosystem functioning globally (Tylianakis et al. 2008, Schweiger et al. 2010, Mohan et al. 2014, Classen et al. 2015, Farrer et al. 2015). For example, increased competition with invasive plant species can exacerbate abiotic effects of global warming and result in species extinctions (Brook et al. 2008). However, interactions with invasive species are rarely incorporated into spatially explicit ecological forecasts (Watling et al. 2013, Weber et al. 2017). This is, in part, because the impacts of climate and species interactions on species' distributions typically operate at different scales (Bullock et al. 2000, Copenhaver-Parry and Bell 2018). Consequently, climatic and biotic interactions have largely been dealt with separately by two bodies of work, Species Distribution Modelling (SDM) and community ecology, respectively (Pollock et al. 2014). SDMs are generally used to forecast species' distributions, often under changing climates (Watling et al. 2013, Fordham et al. 2014). In contrast, exploring how species interactions shape species across large spatial extents have largely been investigated through the lens of community assembly, which is inferred using co-occurrence matrices derived from field-collected data (Pollock et al. 2014). Recently, attempts to integrate these two bodies of work have increased, leading to modelling methods such as such as Joint Species Distribution Models (JSDM; Pollock et al. 2014) .

Invasive plant species that successfully outcompete native species may limit their distributions (Bullock et al. 2000, Bjerknes et al. 2007, Isaac and Williams 2013), however most commonly the effect of invasive species is to decrease of the density of native species in the landscape (Powell et al. 2013). Decreasing density nevertheless has important implications for population persistence (Sexton et al. 2009). Consequently, substantial effort has been made to predict the geographic areas at risk of invasion using SDM (Jimenez-Valverde et al. 2011). Building on this work, JSDMs implement a hierarchical modelling structure in which climatic factors are used to predict suitable habitats while residual variance is partially explained by cooccurrence patterns with other species (Pollock et al. 2014). Although JSDMs are a useful tool 
for investigating the impact of species invasions, they are occurrence based, whereas the impact of invasive species is likely to be determined by invader density (Kulhanek et al. 2011, Bradley 2013). Invasive species modelling studies have been criticised for implicitly equating risk of invasion with risk of impact, with few studies attempting to explicitly model where invasive species presence is likely to elicit ecological response (i.e. 'impact niche' sensu Bradley 2013). The densities of plant species are rarely modelled directly because spatially explicit abundance data are scarce (Nielsen et al. 2005, Van Couwenberghe et al. 2013) particularly at sufficiently large spatial scales to apply SDM approaches (Bradley 2013). In addition, despite efforts to standardise collections, currently available data on species densities use inconsistent methodologies (Bradley et al. 2018). As a result, models predicting the occurrence of invasive species (hereafter, 'occurrence niche') are prolific (Nielsen et al. 2005) but of limited use to conservation managers. Models that incorporate species densities would allow better predictions of changes to native species densities and ranges and are therefore pivotal to improving conservation management planning (Nielsen et al. 2005, Kulhanek et al. 2011).

A link between occurrence-driven SDM predictions and density may be derived from the abundant-centre hypothesis, first formulated by Brown (1984). If similar environmental variables act to define both species distributions and population demography (Weber et al. 2017) then the highest density should occur in the centre of the ecological niche space (Van Couwenberghe et al. 2013, Dallas et al. 2017). Van Couwenberghe et al. (2013) found support for the abundant-centre distribution by modelling 243 plant species, observing peak abundance of most plant species in optimal environmental conditions. However, the success of occurrencedriven climate envelope models for correctly predicting species density (hereafter, 'environmental suitability-density relationship') is variable (Nielsen et al. 2005, Veloz et al. 2015, Dallas et al. 2017), with some studies reporting good fits (Van Couwenberghe et al. 2013, Weber et al. 2017), while other studies find variable or poor predictions (Bradley 2013, 2016). This may be because relationships between species and the environment are typically so-called 'triangular constraint envelopes'; environmental variables universally constrain species' densities to low levels at the edge of the species' environmental niche, however highly climatically suitable areas may have high or low densities, depending on many non-climatic 
factors, such as source-sink dynamics, species interactions, dispersal, and time from colonisation (VanDerWal et al. 2009, Weber et al. 2017). Appropriate statistical tools, such as quantile regressions, yield better results than least squares regression in these cases (VanDerWal et al. 2009, Weber et al. 2017).

Here, we couple modelling with a large-scale field study to explore the distribution of a native species, Chionochloa rubra ssp. rubra (Poaceae) under current and potential future climates, as well as in response to plant invasion, in Tongariro National Park (TNP), New Zealand. This project was carried out in collaboration with Ngāti Rangi, a local iwi (tribe) as part of a larger body of work aimed at restoring capacity to act as kaitiaki (guardians) of TNP by strengthening the connections between Ngāti Rangi and the science underpinning conservation management in TNP. As part of this process, we ran interviews with pāhake (elders) and others with mōhiotanga (knowledge holders). One justification for the selection of C. rubra as a focal species is its status as taonga tipu (treasured plant species) by local indigenous people. In this chapter, we use $C$. rubra as an example species to illustrate a novel modelling methodology. However, as part of a larger body of work, we modelled the responses of four other taonga tipu to climate change and plant invasion- Anisotome aromatica, Coprosma perpusilla, Dracophyllum recurvum and Gentianella bellidifolia. The modelling outcomes for these other species are provided in Appendix 4.

Here, we model both climate and pairwise density dependence of $C$. rubra with an invasive species (Calluna vulgaris (L.) Hull; Ericaceae) in a novel hierarchical framework. First, we use occurrence data to calibrate climate envelope models for both species. We hypothesise $\left(\mathbf{H y p}_{1}\right)$ that under projected future climate in TNP, the geographic distribution of $C$. rubra will decrease, while the distribution of $C$. vulgaris will expand. Second, we use field collected density data to identify density thresholds of $C$. vulgaris at which $C$. rubra densities were significantly reduced. We hypothesise $\left(\mathbf{H y p}_{\mathbf{2}}\right)$ that high $\mathbf{C}$. vulgaris densities are associated with low C. rubra densities. Third, we relate local densities of C. vulgaris to environmental suitability, derived from its occurrence-driven climate envelope model using quantile regression. We hypothesise $\left(\mathrm{Hyp}_{3}\right)$ that environmental suitability is predictive of the upper limit of $C$. vulgaris densities. Finally, we integrate these analyses to make risk projections for $C$. rubra. We divide 
the geographic extent of the park under both the current and projected future climate into four categories - a) climatically unsuitable for C. rubra, b) climatically suitable for $C$. rubra with no overlap with $C$. vulgaris, c) climatically suitable for $C$. rubra and overlapping with the occurrence niche distribution of $C$. vulgaris, and d) climatically suitable for $C$. rubra and overlap with the impact niche distribution of $C$. vulgaris. We hypothesise $\left(\mathbf{H y p}_{4}\right)$ an increasing overlap between the distribution of $C$. rubra and the impact niche distribution of $C$. vulgaris under future climate predictions. 


\section{Methods}

\section{Study system}

Tongariro National Park (TNP) is an alpine conservation area in the central North Island of New Zealand $\left(-39.25^{\circ} \mathrm{N}, 175.58^{\circ} \mathrm{E}\right)$. The park is currently $1.5^{\circ} \mathrm{C}$ warmer and drier $(-5 \mathrm{~mm}$ of rain/year) than it was 50 years ago and the climate is predicted to continue changing (IPCC 2014), threatening the persistence of native alpine communities (Halloy and Mark 2003). An additional driver of ecological change in TNP is the invasion of Calluna vulgaris (L.) Hull (European heather; Ericaceae); a non-native species that was introduced to the park in 1912 (Chapman and Bannister 1990, Rogers and Leathwick 1996). C. vulgaris invasion into communities reduces the occurrence, density (Keesing 1995), and sexual reproduction (Chapter 2) of native plant species. Tall tussock grasslands are a distinct vegetation type (Steer and Norton 2013) that were historically prevalent throughout TNP (Atkinson 1985). Such grasslands were dominated by $C$. rubra but have largely given way to $C$. vulgaris dominated shrubland in many areas (Rogers and Leathwick 1996). In uninvaded communities, C. rubra provides ecosystem structure and facilitates the growth of other native plant species (Reitsma 1994). The distributions of both $C$. vulgaris and C. rubra are likely to be climatically driven, with occurrences strongly controlled by elevation (Steer and Norton 2013).

\section{Density surveys}

We estimated canopy cover (\%) of both C. rubra and C. vulgaris within $1 \mathrm{~m}^{2}$ GPS tagged quadrats around TNP $(n=490)$. These measurements were carried out during two discrete sampling efforts. 1) In 2016, we established transects along fourteen sampling locations, within three elevational transects in the northwest, west and east of the park. Sampling locations along the transects were spaced at 100 vertical metre intervals, from the lowest elevations at the park boundary (900-1000 m a.s.I.) to the elevational limit of C. vulgaris (1300 to $1500 \mathrm{~m}$ a.s.I.). Ten $1 \mathrm{~m}^{2}$ plots were surveyed at each location and canopy cover of all species recorded. Placement of plots was systematic within a $500 \mathrm{~m}^{2}$ Whittaker plot design, following (Powell et al. 2013). 2) In 2018, we surveyed along four elevational transects in the north, south, southeast and northeast of the park. These transects generally followed established trails, with surveys conducted a minimum of 50m from the trail, approximately every 50 vertical metres, 
from the periphery of the park to the highest elevation at which vegetation was found (up to $2240 \mathrm{~m}$ a.s.l.). Additional survey locations along the periphery of the park were included in order to adequately sample the extensive lowlands. At each survey location, a $23 \mathrm{~m}$ transect was laid perpendicular to the slope, over the most continuous vegetation patch found; five $1 \mathrm{~m}^{2}$ plots were randomly placed along the transect and were assessed for canopy cover of $C$. vulgaris and C. rubra.

\section{Climate envelope modelling}

To address $\mathrm{Hyp}_{1}$, that the distribution of $C$. rubra will contract and the distribution of $C$. vulgaris will expand in response to predicted changes in climate, we built ensemble SDMs for both species. We chose to use ensemble models because previous studies speculate that they may be most suitable for environmental suitability-density applications (Weber et al. 2017). Ensemble models may better describe the margins of environmental suitability because they provide a good indication of uncertainties (Araújo and New 2007). Our study is, to our knowledge, the first to have applied ensemble modelling for this purpose. Occurrence data used to calibrate climate envelope models were drawn from the described field surveys, from the Global Biodiversity Information Facility (GBIF; www.gbif.org) and from the Botanical Society of Britain and Ireland (BSBI; https://bsbi.org/) and supplemented with occurrence records collected throughout TNP in the years 2015-2018 $(n=118,734$ and 1905 for $C$. vulgaris and $C$. rubra, respectively). Occurrences were drawn for C. rubra from several protected areas on the North Island of New Zealand (Supplementary Figure 14) using the Protected Area Layer (retrieved from www.data.linz.govt.nz in May 2018). Boundaries were delineated with the addition of a with a three-kilometre buffer to include occurrences in similarly vegetated habitats which commonly occur just outside the parks. We calibrated the climate envelope for C. vulgaris within TNP (with a $3 \mathrm{~km}$ buffer, as for $C$. rubra) as well as for a subset of its native range in Europe. To reduce observer bias, we included only those areas of the native range within which the occurrence of $C$. vulgaris appeared to be widespread (Bradley 2016); a strong sampling bias was evident across the native range of $C$. vulgaris whereby Western Europe had significantly more occurrence records than Eastern Europe and the margin of densely surveyed areas was clearly delineated by political borders. The total number of occurrences used to 
calibrate the final model was 5971 for $C$. vulgaris and 169 for C. rubra. All public repository data with low geographic precision were removed from the dataset. The dataset was partially corrected for observer bias by limiting observations to one per 30 arcsecond grid cell (Dormann et al. 2012). The geographic extent of calibration was limited for both species in ARCGIS 10.4.1 (ESRI 2011) by cropping environmental predictor layers. We downloaded current and future climatic data as bioclimatic variables (BIOCLIM; retrieved from www.worldclim.org/bioclim in 2017) raster layers at a 30 arcsecond resolution (Hijmans et al. 2005). Current climatic conditions are based on interpolations of observed climatic data from 1960-1990. To describe future climates, we averaged the predictions of five Global Circulation Models (GCMs; HadGEM2-ES (MOHC: UK), GISS-E2-R (NASA-GISS: USA), GFDL-CM3 (NOAA-GFDL: USA), BCCCSM1.1 (BCC: China) and NorESM1-M (NCC: Norway)) for 2070, separately for representative concentration pathways (RCP) 4.5 and 8.5. GCM choice was based on the ability to validate present climate data in New Zealand, as well as to encompass the maximum variation in projections (Mullan et al. 2018).

Models were calibrated in R version 3.5.1 (R Development Core Team 2016). To inspect collinearity among BIOCLIM predictor layers, we used the function 'prcomp' in the package 'factoextra' version 1.0.5 (Kassambara and Mundt 2017) to conduct a principal component analysis. Predictors with low variability over the calibration range were excluded. We calculated a series of preliminary models for each species in the 'biomod2' package, version 3.3.7 (Thuiller et al. 2009). We used one set of environmentally stratified pseudo-absences (a measure of the available environmental conditions; Barbet-Massin et al. 2012) in preliminary models ( $n=2985$ and 84 for $C$. vulgaris and C. rubra, respectively). We modelled with the following algorithms: Artificial Neural Network (ANN; Ripley 1996), Generalized Additive Model (GAM; Hastie and Tibshirani 1990), Generalized Linear Model (GLM; McCullagh and Nelder 1989), Multiple Adaptive Regression Splines (MARS), Random Forest (RF; Breiman 2001) and Surface Range Envelope (SRE/BIOCLIM; Busby 1991). We included all predictor variables in the first preliminary model and excluded colinear variables in a stepwise fashion in subsequent models based on variable importance. Variable importance was calculated as an average from three permutations within the 'BIOMOD_Modeling' function for each modelling algorithm 
independently. The two most important, uncorrelated variables were included in the final model.

Ten sets of environmentally stratified pseudo-absences were selected to build the final model. Each algorithm was run three times with a 70:30 split of the data for each pseudoabsence dataset; and once on each full pseudo-absence dataset. This resulted in 240 individual models. Individual models that satisfied a validation criterion of Receiver Operating Characteristic curve $(\mathrm{ROC})>0.7$ were included in a model ensemble and mean-weighted. We projected the final ensemble model of $C$. vulgaris and $C$. rubra distributions in current and predicted future climates in TNP. We transformed probabilistic ensemble model projections of C. rubra and C. vulgaris distributions under the current climate into binary predictions. We used the 'roc' function in the package 'pROC' (Robin et al. 2011) to build a ROC curve with probabilistic predictions at each of our field survey points as the predictor and true presence/absence data from our field surveys as the response variable. We chose thresholds from the 'local maximas' provided by pROC, prioritising the sensitivity of both models.

\section{Density-dependence}

To address $\mathrm{Hyp}_{2}$, that high densities of $\mathrm{C}$. vulgaris are associated with lower densities of C. rubra than expected based on climate information alone, we used co-occurrence patterns derived from the canopy cover of the two species within $1 \mathrm{~m}^{2}$ quadrats. We subset the total survey data $(n=490)$ to only include those survey locations where presence was predicted by climate envelope models for both species $(n=310)$. Both species were absent from a substantial portion of the surveyed plots $(n=103)$, which may reflect small-scale environmental conditions such as inadequate soil or frequent disturbance that excluded both species. We also excluded plots where $C$. vulgaris was absent, to decrease the effect of dispersal limitation. The remaining data subset $(n=77)$ was used in the following analyses. Co-occurrence data were analysed using a Boosted Regression Tree (BRT) in the package 'gbm' (Greenwell et al. 2018). A typical feature of ecological datasets is non-linear data (De'ath and Fabricius 2000), particularly when global environmental change is incorporated (Tylianakis et al. 2008). BRT is a useful technique to explore these data, particularly as it is robust to permutations in the training data (Iverson et al. 2004) and the thresholds it produces are useful for land managers (Panetta and 
Gooden 2017). We followed Elith et al. (2008) to calibrate the BRT. The model was calibrated with a learning rate of 0.01 , a bag fraction of 0.5 and a step size of 0.1 . As the purpose of the analysis was to identify thresholds rather than closely model the data, tree complexity was set to 1 . The BRT was poorly descriptive, therefore the viability of the partition identified by the BRT was further supported with Wilcoxon-signed rank tests using the function 'wilcox.test'. We expect that the poor descriptive power of our BRT is primarily a result of zero-inflated data, which is typical of ecological datasets (Bellvé 2018).

\section{Environmental suitability-density relationship}

Our third hypothesis $\left(\mathrm{Hyp}_{3}\right)$ was that the upper limits of $C$. vulgaris densities can be predicted from climatic suitability predictions derived from a presence-driven climate envelope model. We extracted environmental suitability scores predicted by the $C$. vulgaris climate envelope model for all quadrat locations. We derived a series of relationships between $C$. vulgaris environmental suitability and percentage cover using quantile-regression in the 'quantreg' package version 5.36 (Koenker et al. 2018). We calculated regressions for the $50^{\text {th }}$, $60^{\text {th }}, 70^{\text {th }}, 80^{\text {th }}, 90^{\text {th }}$, and $97^{\text {th }}$ quantiles. We determined standard error for each quantile using 200 bootstraps and calculated Nagelkerke's $r^{2}$ to estimate predictive power, using the ' $r q^{\prime}$ function in the package 'rcompanion', version 1.13.2 (Mangiafico 2018). Nagelkerke's $r^{2}$ is more appropriate for quantile regression than traditional $r^{2}$ values (VanDerWal et al. 2009).

\section{Prediction}

Our final hypothesis $\left(\mathbf{H y p}_{4}\right)$ was that under all climate scenarios, a large proportion on the geographic space climatically available to C. rubra is also climatically suitable for high densities of $C$. vulgaris (within the impact niche). We transformed climate model projections of C. vulgaris distributions under the current and future climates into three partitions, based on two thresholds. The first partition was derived as described above. The purpose of this first partition was to delineate areas in which $C$. vulgaris does and does not occur: its occurrence niche. The purpose of the second partition was to delineate areas in which it is possible for $C$. vulgaris to reach high densities that are associated with depressed densities of $C$. rubra: its impact niche. To achieve this, we used the 'predict.rq' function in the 'quantreg' package. We predicted the environmental suitability value associated with the $C$. vulgaris density threshold 
identified by BRT, as defined by the $90^{\text {th }}$ quantile regression. Finally, we overlaid $C$. rubra spatial projections with spatial projections of the occurrence and impact niches of $C$. vulgaris. We calculated the proportion of TNP land area (as pixel numbers) corresponding to each combination of predictions for the two species. 


\section{Results}

\section{Climate envelope modelling}

The ensemble climate envelope models for $C$. vulgaris and $C$. rubra performed well. The C. vulgaris model had a ROC of 0.98 , a sensitivity of 0.94 and a specificity of 0.96 . C. vulgaris was most responsive to mean temperature of the warmest quarter (variable importance $=$ 0.93); diurnal temperature variability also explained some variance (variable importance $=$ 0.13). Under the current climate, C. vulgaris is climatically limited only at high elevation; 586.3 $\mathrm{km}^{2}$ of the $796 \mathrm{~km}^{2}$ park is climatically suitable for the occurrence of this species. In 2070 we predict an expansion of this occurrence niche at higher elevations and no contraction at lower elevations. We predict that an additional $154 \mathrm{~km}^{2}$ of the park will become climatically suitable for $C$. vulgaris occurrence under RCP 4.5 and $183.6 \mathrm{~km}^{2}$ under in 2070 under RCP 8.5. The $C$. rubra ensemble climate model also performed well, with a ROC of 0.99 , a sensitivity of 0.94 and a specificity of 0.98. C. rubra was most responsive to mean temperature of the wettest quarter (variable importance $=0.87$ ). Total precipitation of the wettest quarter was also predictive (variable importance $=0.15$ ). Our model indicates that $C$. rubra occurrence is climatically limited at both high and low elevations under the current climate. In 2070, we predict a small upslope expansion of this species, and a significant contraction of its range at low elevations, resulting in an overall decrease in climatically available geographic area. Under the current climate, 167.2 $\mathrm{km}^{2}$ of TNP is climatically unsuitable for this species; in 2070 under RCP 4.5 and 8.5, an additional $152.7 \mathrm{~km}^{2}$ and $328.8 \mathrm{~km}^{2}$ of space becomes climatically unavailable, respectively.

\section{Density dependence}

We hypothesised that high $C$. vulgaris density is associated with depressed C. rubra density $\left(\mathbf{H y p}_{2}\right)$. We record a negative density-dependence correlation between $\mathbf{C}$. rubra and $\mathrm{C}$. vulgaris (Figure 4.1A). The final BRT was built with 50 trees and identified tipping points in this relationship at $77.5 \%$ and $32.5 \%$ C. vulgaris cover, categorising C. vulgaris density into 'low' (< $32.5 \%$ cover), 'medium' (32.5\% - 77.5\% cover) and 'high' (> 77.5\% cover; Figure 4.1B). The predictive power of this analysis was poor, explaining only $5.01 \%$ of total variance. However, the difference between the three partitions was supported by Wilcoxon rank sum tests. Plots with low $C$. vulgaris cover have an average $C$. rubra cover of $6.75 \%$. In comparison, average 
cover of $C$. rubra in plots with medium C. vulgaris cover was significantly less $(3.06 \%$; $W=968.5$, $\mathrm{p}<0.001$ ). Where $C$. vulgaris density was high, average $C$. rubra cover was dramatically changed, with an eight-fold decrease relative to plots with low $C$. vulgaris density $(0.84 \% ; \mathrm{W}=$ $489, p<0.001)$. We therefore chose this threshold as the density delineating the impact niche of $C$. vulgaris.
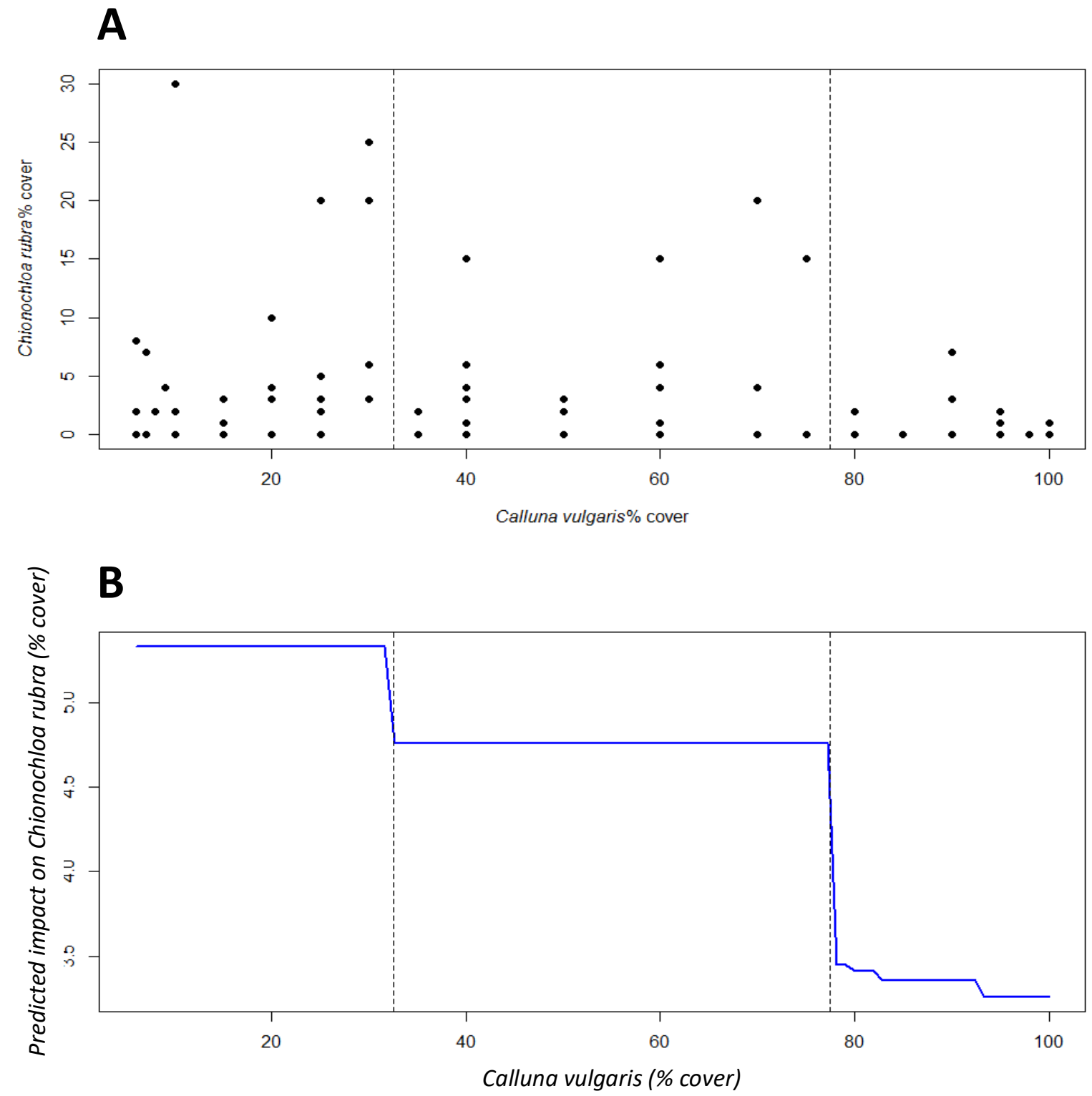

Figure 4.1: Density dependence of Chionochloa rubra and Calluna vulagris: A) Canopy cover of C. rubra as a function of $C$. vulgaris canopy cover; B) predictions of the impact of $C$. vulgaris canopy cover on $C$. rubra canopy cover from the Boosted Regression Tree analysis. Dashed lines in both plots indicate major thresholds identified by the analysis ( 77.5 and $32.5 \%$ cover). 


\section{Environmental suitability-density relationship}

Our third hypothesis was that the upper limit of $C$. vulgaris density can be modelled using occurrence-derived environmental suitability. We found strong support for a triangular constraint envelope environmental suitability-density relationship for $C$. vulgaris (Figure 4.2). Both least squares regression and all quantile regressions showed a positive relationship between predicted environmental suitability and C. vulgaris density (Table 4.1). However, explanatory power was highest for upper quantiles: at the $90^{\text {th }}$ percentile, $65 \%$ of variance in $C$. vulgaris density was explained by predicted environmental suitability, significantly more than the $20 \%$ explained by ordinary least squares regression $(t=10.84, p<0.001)$.

Table 4.1: Performance of six quantile regressions in predicting the environmental suitabilitydensity relationship. Quantile regressions model Calluna vulgaris canopy cover based on environmental suitability scores predicted from an ensemble climatic model in Tongariro National Park, New Zealand.

\begin{tabular}{|r|r|r|r|r|r|} 
Quantile & Estimate & \multicolumn{1}{|c|}{ Standard Error } & \multicolumn{1}{c|}{ t value } & $\boldsymbol{p}$ value & Nagelkerke $\boldsymbol{r}^{\mathbf{2}}$ \\
\hline 0.50 & 0.003 & 0.002 & 2.181 & 0.030 & 0.020 \\
\hline 0.60 & 0.011 & 0.005 & 2.064 & 0.040 & 0.112 \\
\hline 0.70 & 0.027 & 0.005 & 5.764 & $<0.001$ & 0.330 \\
\hline 0.80 & 0.052 & 0.007 & 7.188 & $<0.001$ & 0.520 \\
\hline 0.90 & 0.080 & 0.007 & 11.390 & $<0.001$ & 0.653 \\
\hline 0.97 & 0.101 & 0.002 & 42.735 & $<0.001$ & 0.730 \\
\hline
\end{tabular}

\section{Prediction}

Our fourth hypothesis was that under all climate scenarios, a large proportion of the distribution of $C$. rubra overlaps with the impact niche of $C$. vulgaris. Under the current climate, $486.4 \mathrm{~km}^{2}$ of the modelled distribution of $C$. rubra in TNP overlaps with the impact niche of $C$. vulgaris (Figure 4.3). Under RCP 4.5 and 8.5 in 2070 we predict that the impact niche distribution of $C$. vulgaris will cover a $442.4 \mathrm{~km}^{2}$ and $289.0 \mathrm{~km}^{2}$ area of TNP, respectively. Under future climate scenarios, our model integrating C. vulgaris density predicted as much as 595.1 $\mathrm{km}^{2}$ and $617.7 \mathrm{~km}^{2}$ (under RCP 4.5 and 8.5 , respectively), will be either climatically unavailable to $C$. rubra or affected by overlap with the impact niche of $C$. vulgaris. 


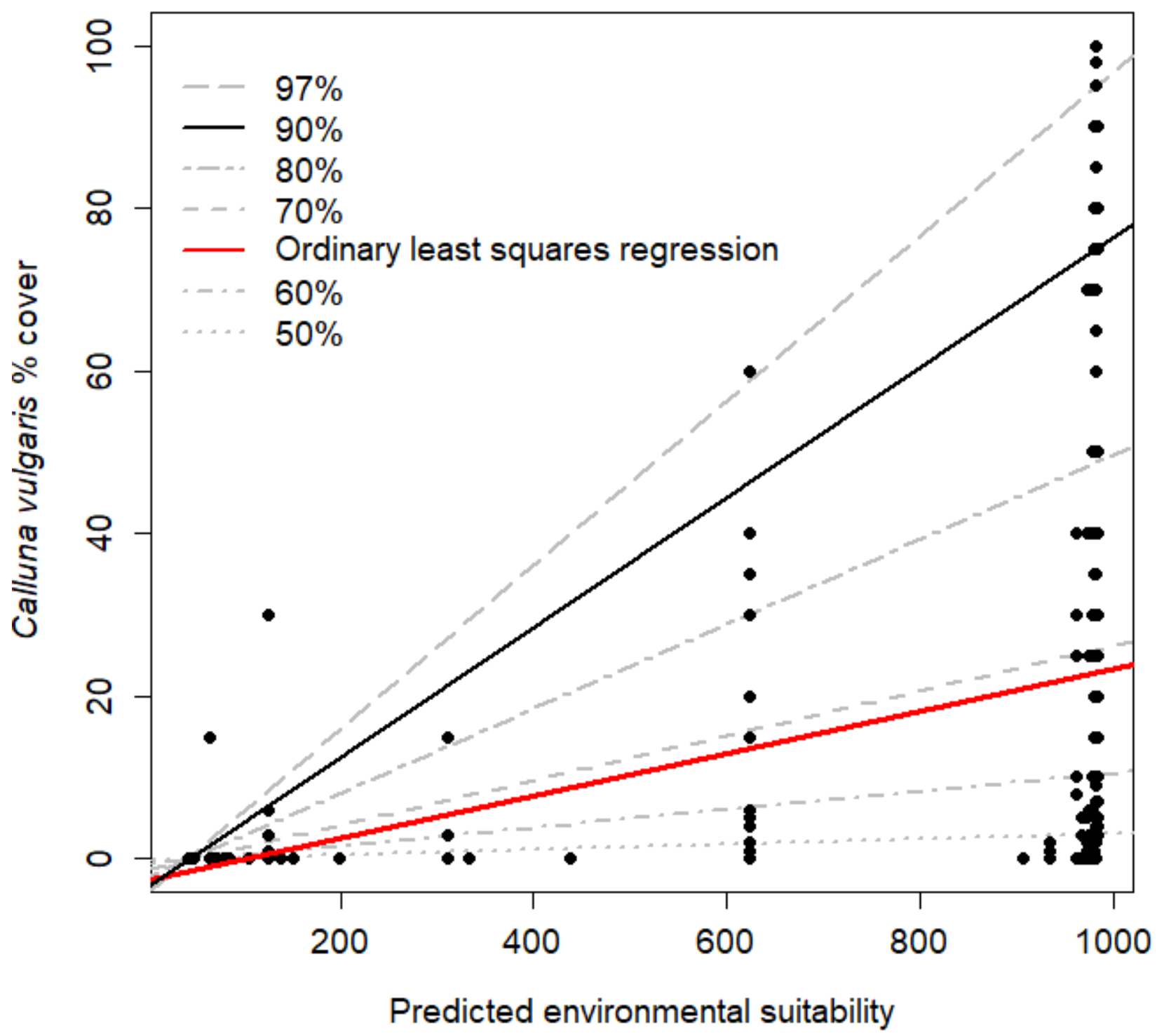

Figure 4.2: Density of Calluna vulgaris as a function of ensemble climatic model suitability scores, presented with quantile regressions. Lines indicate the relationship as estimated by ordinary least squares regression (red line) and $97^{\text {th }}, 90^{\text {th }}, 80^{\text {th }}, 70^{\text {th }}, 60^{\text {th }}$ and $50^{\text {th }}$ quantile regressions (grey and black lines). Nagelkerke pseudo $r^{2}$ values for each relationship is provided in the figure legend. 


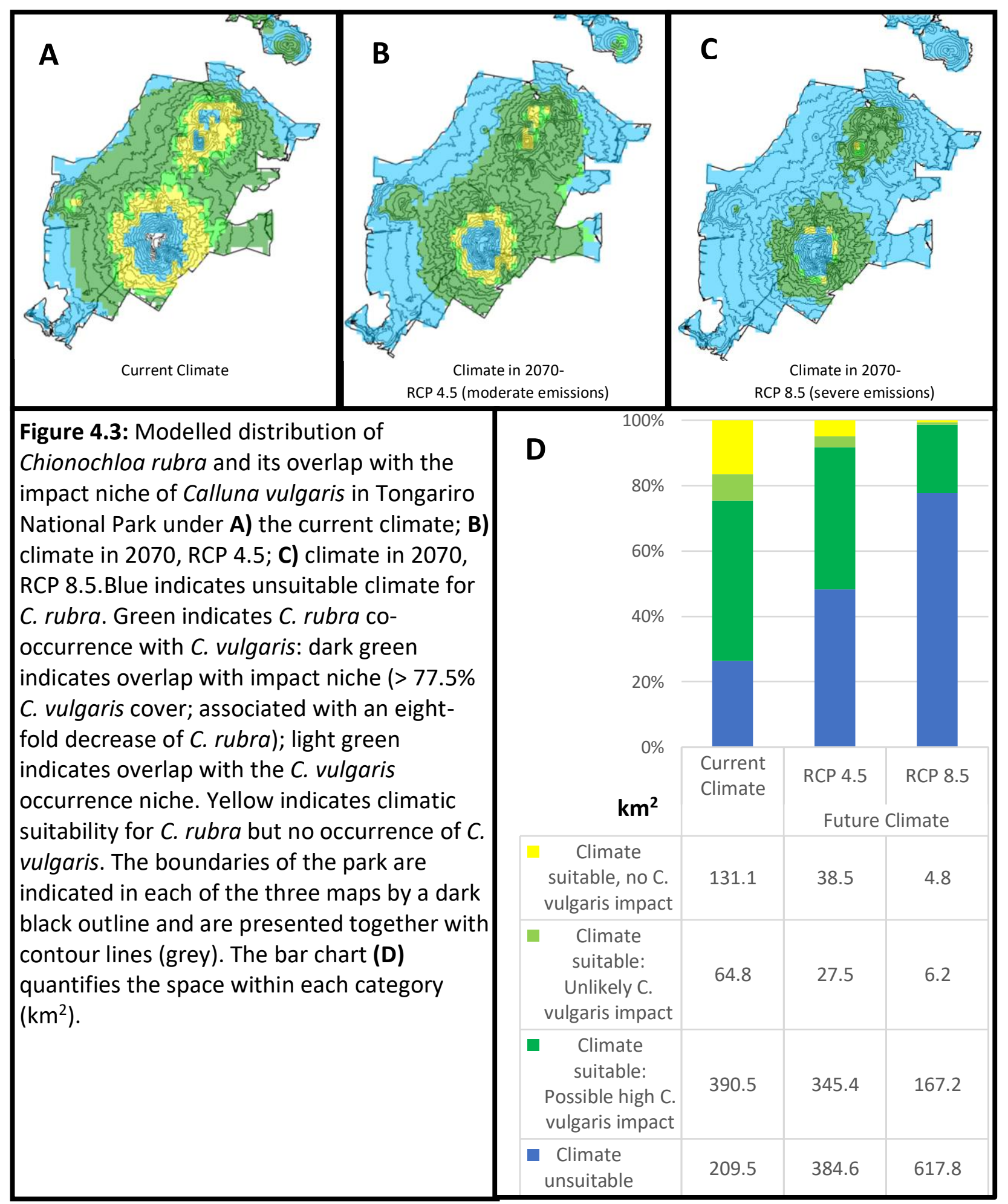




\section{Discussion}

In this study, we modelled the distribution of a native plant species, C. rubra, under future climate change, including pairwise density-dependent competition with the invasive $C$. vulgaris. In congruence with $\left(\mathbf{H y p}_{\mathbf{1}}\right)$, we found strong support for climatic limitation of $\mathbf{C}$. rubra at both the upper and lower elevational limits of its range and we predicted a range contraction under future climates. C. vulgaris was limited only at the lower elevational limit of its range and we predicted a range expansion under warmer climates. In support of $\mathbf{H y p}_{\mathbf{2}}$ we found a negative density dependence between $C$. rubra and $C$. vulgaris, suggesting competitive impacts of $C$. vulgaris on C. rubra. We identified a C. vulgaris density threshold of $77.5 \%$; $C$. vulgaris densities exceeding this threshold were associated with an eight-fold decrease in the mean density of $C$. rubra. Further, we found support for $\mathrm{Hyp}_{3}$ : at the $90^{\text {th }}$ percentile, $65 \%$ of the variance in $C$. vulgaris density was explained. Finally, to address $\mathbf{H y p}_{4}$, we quantified the effect of climate change on the distribution of $C$. rubra as well as on the degree of overlap with the distribution of $C$. vulgaris impact niche and we find that this overlap increases. This novel combination of methods from species distribution modelling and community ecology allowed us to predict both the direct effect of climate on C. rubra and its indirect effect, mediated by the changing distribution of the impact niche of $C$. vulgaris. As a result, we offer a more accurate representation of the potential risk for $C$. rubra in TNP in a warming climate.

Although the range contraction of native alpine species (Halloy and Mark 2003) and the expanding ranges of lowland species and exotics into alpine habitats (Dainese et al. 2017) are both commonly predicted consequences of climate change that may affect the persistence of alpine species, they are often not considered together in modelling studies (Watling et al. 2013, Wisz et al. 2013; but see e.g. Pollock et al. 2014). We observed a range contraction of $C$. rubra in projected future climates relative to current day conditions as a direct effect of climate. This range contraction occurs primarily at lower elevations and is in line with global predictions (Engler et al. 2011). Taken alone, this direct effect of climate indicates increased risk of extirpation of $C$. rubra in TNP. We concurrently predicted a range expansion of $C$. vulgaris to higher elevations within the park. The response of $C$. vulgaris to a changing climate includes the geographic expansion of both its occurrence niche and its impact niche at the leading edge. The 
latter occurs as the climate at the upper limits of its elevational distribution become more suitable for high density stands. By modelling the impact niche, we contribute in a novel way to the risk assessment of $C$. rubra. Namely, we find that although contractions of the distribution of $C$. rubra that are driven by climate change are significant, the increasing overlap between $C$. rubra distribution and the impact niche distribution of $C$. vulgaris has the potential to adversely affect $C$. rubra over a greater spatial extent. The proportion of the predicted climatically available space where $C$. rubra may be affected by density-dependent competition from $C$. vulgaris rises from $77 \%$ to $96 \%$ under the current climate scenario and in 2070 under a RCP 8.5 scenario, respectively. An increasing prevalence of competitive effects is expected in alpine ecosystems generally, and is largely driven by the geometry of mountains, where less space is available at higher elevations (Körner 2007). Notably, alpine species are typically stress-adapted and are therefore poor competitors in less stressful abiotic conditions (Grime 1974). These characteristics suggest that the need to quantify risk to species resulting from the expansion of the impact niche distribution of invasive species is particularly acute in alpine ecosystems. However, such changes may occur in other ecosystem types. For example, the overlap of species of interest with the impact niche of invasives may occur where the latter are expanding their distribution in their novel range. It is crucial that such species interactions are incorporated into SDM more frequently (Wisz et al. 2013).

The occurrence niche of invasive species is often modelled (Jimenez-Valverde et al. 2011) however models of impact niche are rare (Bradley 2013). Attempts to model impact niche are generally limited by data availability (Nielsen et al. 2005, Van Couwenberghe et al. 2013). Therefore, we developed a novel modelling approach by utilizing the relationship between occurrence-driven environmental suitability and density of the invasive species. This allowed us to make use of a large pre-existing occurrence dataset ( $n=5971)$, and supplement this with a comparatively smaller field collected density dataset $(n=77)$ to predict impact niche. Furthermore, we model invasive impact using density-dependent responses, which are preferable to techniques employing co-occurrence for two reasons. Firstly, density data should predict invasive impact more accurately than species' presence (Kulhanek et al. 2011). For example, we demonstrate significant geographical extents over which $C$. vulgaris and $C$. rubra 
are expected to co-occur but in which we predict no significant competitive interactions. Secondly, the primary competitive effect of invasives on native species may be to reduce their density rather than occurrence (Copenhaver-Parry and Bell 2018). For example, in our system we find consistent $C$. rubra and $C$. vulgaris co-occurrence, despite known competitive interactions between the species leading to its decreased abundance in the landscape (Keesing 1995, Rogers and Leathwick 1996). A co-occurrence matrix would have been insufficiently sensitive to detect these responses. Consequently, relative to co-occurrence, our densitydependent approach should more accurately identify the areas where competition is likely to occur by (1) excluding areas where competitive effects are unlikely and (2) being sensitive to competitive effects other than complete exclusion.

The correct interpretation of model outputs is a prerequisite for their utility in conservation management (Araújo and Townsend Peterson 2012). The outputs of this modelling approach indicate the upper limits of $C$. vulgaris density rather than its average density. Modelling the upper limits of density is ecologically meaningful, as climatic variables may determine upper limits of density at coarse spatial scales without fully explaining density in all places (VanDerWal et al. 2009, Van Couwenberghe et al. 2013). We therefore predict the potential impact of climate change and invasion on C. rubra. For example, given the model predictions in this study we do not expect that $C$. vulgaris will homogenously attain densities of $>77.5 \%$ over a $486.4 \mathrm{~km}^{2}$ area under the current climate. Instead, we predict that this area has the climatic potential to host such dense stands. This potential is likely to be unrealised in many areas at small spatial scales, because factors such as soil characteristics, microclimate or disturbance regime limit species densities at smaller spatial scales. The conflation of potential and certain impact by climate envelope model users is a common concern with all climateenvelope SDM approaches (Araújo and Townsend Peterson 2012). Nevertheless, the approach provides important information to land managers. For example, areas of minimal impact may be identified, precluding the waste of management resources in these areas. Alternatively, managers could delineate containment boundaries based on maximum impact risk. Finally, understanding threshold densities of the invader at which an ecological response is elicited can 
enable managers to prioritise control to reduce densities below this threshold (Bradley 2013), thus maximising conservation benefits for native species.

Our approach is inappropriate for modelling the geographic range of an invasive species that has not yet reached high densities in portions of its novel range. Where invasive species have not gone through the exponential phase of population growth (Theoharides and Dukes 2007), their density is more likely to reflect dispersal limitation and time-since-arrival than potential density based on climatic variables (Theoharides and Dukes 2007, VanDerWal et al. 2009, Weber et al. 2017). In these cases, our approach may underestimate invasive effects on native species. Further, species in the early stages of invasion are likely to have small geographic ranges (Theoharides and Dukes 2007). This may restrict the power of analyses, particularly if populations do not span a wide scope of the driving environmental gradients (Van Couwenberghe et al. 2013, Knouft 2018). Two important criteria of our study system made the application of this hierarchical density-dependent modelling approach viable. Firstly, C. vulgaris' environmental suitability scores, derived from the occurrence models, accurately predicted the local density of this species in the field. Secondly, the invasive species' impact on native species could be predicted by the invasive species' density. We suggest that future applications of our hierarchical density-dependent modelling approach be limited to ecological contexts that meet these two criteria.

The power to detect an environmental suitability-density relationship is variable among studies (Nielsen et al. 2005, Veloz et al. 2015, Dallas et al. 2017), implying that there may be ecological limitations to its implementation. Several ecological conditions that are likely important were met in out model ecosystem. For example, $C$. vulgaris is highly competitive in TNP (Rogers and Leathwick 1996). Dominant plant species' densities may be better predicted by climate envelope models than those of subordinate species, as their distributions are less likely to be affected by competitive exclusion (Dallas et al. 2017). Further, environmental suitabilitydensity relationships may break down if species' distributions and local densities are driven by different variables (Nielsen et al. 2005, Veloz et al. 2015, Bradley 2016, Dallas et al. 2017). For example, in some ecosystems, processes like succession strongly influence species density patterns (Nielsen et al. 2005). In fact, density of species in patchy habitats may be primarily 
driven by non-climatic factors (Van Couwenberghe et al. 2013). Non-climatic factors are expected to augment the local density of species, resulting in the so-called 'triangular constraint envelope' of density-climate relationships (VanDerWal et al. 2009). If important, non-climatic factors may weaken the environmental suitability-density relationship (Nielsen et al. 2005). In these cases, a larger density dataset would be required to validate the trend in the field. Further, their utility would be compromised by a loss of lower predictive power, resulting in type II error in invader impact predictions. Type I error of predictions is only expected if nonclimatic predictors are consistently correlated with climatic variables over geographic space. The influence of non-climatic factors on the strength of the environmental suitability-density relationship should be a research priority before similar approaches can be routinely applied to other ecosystems.

There are several limitations to modelling impact using our density-dependent approach. Firstly, we assume that species' interactions can be deduced from correlative density data. Within our study system, it is likely that negative density dependence of $C$. rubra and $C$. vulgaris is caused by strong competition by $C$. vulgaris. This assertion is supported by historical observations of changes in C. rubra density with C. vulgaris invasion (Rogers and Leathwick 1996). However, an important avenue for future work is to experimentally quantify the effects of pairwise species interactions (Hector and Loreau 2007, McLaren and Turkington 2011). An additional consideration is that the impact of some invasive species may not be related to their density. Our approach is not well suited to evaluate the effects of species with unique characteristics may have large per-capita impacts on ecosystems (Bradley 2013). Finally, our study is based on correlative climate envelope modelling and is subject to the limitations of these approaches. Climate envelope models are static and probabilistic in nature, they assume that correlations between environmental variables are constant, and that variables determining the range of species are independent (Buckley et al. 2010). This assumption is problematic when making dynamic predictions, as is the case when predicting climate change responses (Buckley et al. 2010). Species interactions are likely to vary with climate (Pollock et al. 2014) even where species' canopy cover is consistent (Chapter 3). More empirical research is required to understand species' interactions under a changing climate. For example, the experimental 
removal of species either in conjunction with experimental temperature manipulation or along elevational gradients. The use of experimental data to incorporate mechanism into correlative models should improve the predictive accuracy of such models (Dormann et al. 2012).

SDMs are a leading avenue through which scientists can predict the futures of species of concern, but they do not take into account interactions (Watling et al. 2013), notably those with invasive species. SDMs incorporating the direct impact of climate and its indirect impact, mediated by the changing distribution of the impact niche of invasive species have the potential to substantially add to the accuracy of risk assessments for taxa of conservation concern. Here we implement a novel modelling framework which allows us to model the impact of invasive species by predicting its density and relating this to the density of a native species using large existing occurrence datasets, supported by field data collection. Our study highlights the importance of including species interactions in ecological forecasts, particularly in alpine systems where species are strongly affected by the upward expansion of lowland or exotic species. Modelling studies that investigate risk associated with the competitive impact of invasive species require predictions of their impact niche rather than their occurrence niche. Predictions of density are a promising avenue towards predicting impact niche (Van Couwenberghe et al. 2013). Predictions of invasive species densities and their impact provides useful information to conservation managers. 
5 Inter-island discrepancies in plant invasive success are associated with human disturbance but not environmental filtering

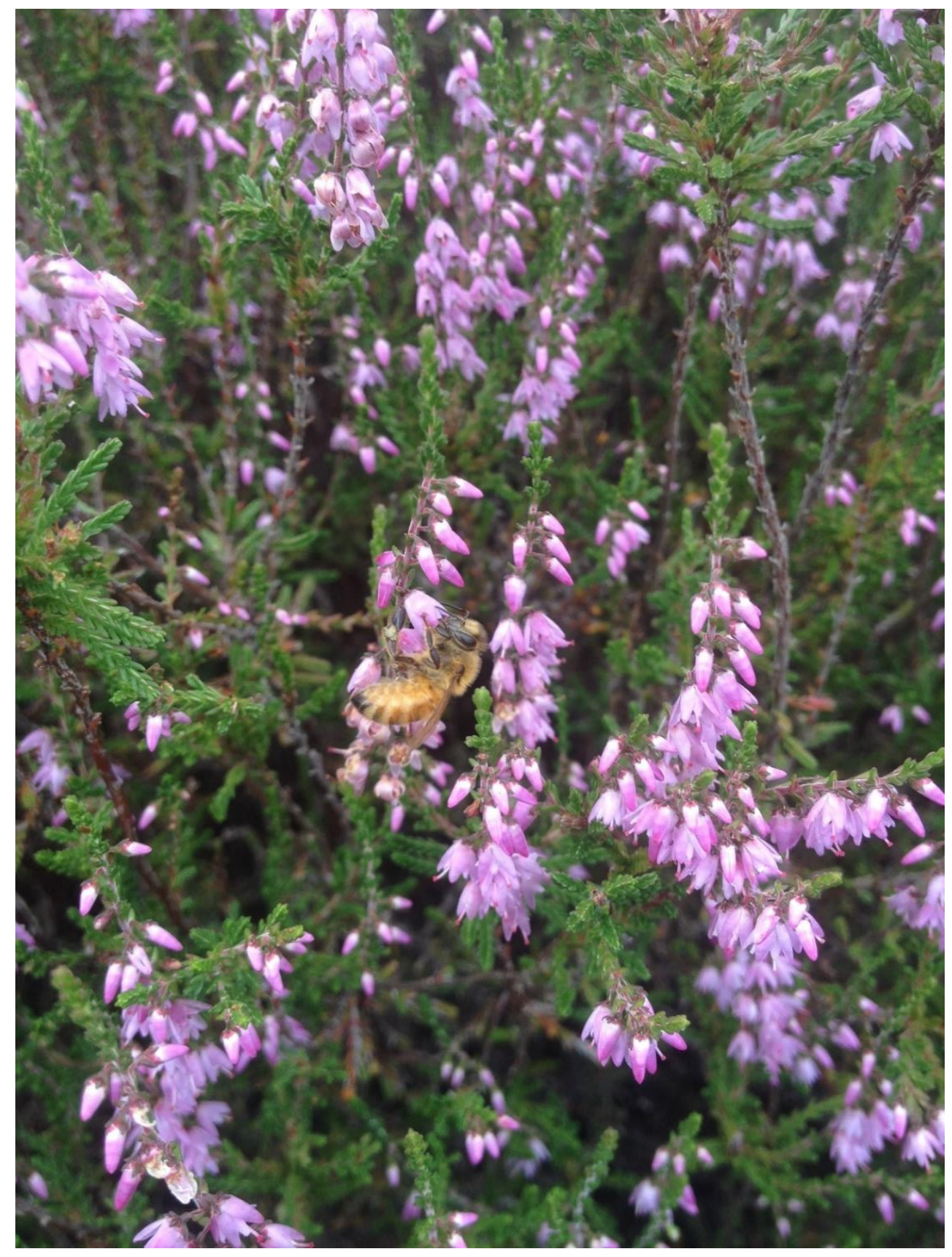

Calluna vulgaris in the Rangipo desert in pollinated by $\underline{\text { Apis }}$ mellifera.

Giejsztowt, 2018 


\section{Introduction}

Why species invade successfully in some areas and not others is a central question in ecology (Theoharides and Dukes 2007, Nuñez et al. 2009, Mainali et al. 2015). The ability to accurately predict species' invasions is increasingly important as rates of species arrival and spread are accelerating globally (Auffret et al. 2014, Horvitz et al. 2017). When species arrive to a new ecosystem their success may be limited by biotic or abiotic filters (Theoharides and Dukes 2007). However, a lack of stochastic events may also limit invasive spread where conditions are otherwise favourable (Simberloff 2009). Including all relevant factors that determine invasive spread success enables better predictions of both the identity of introduced species which are likely to become problematic and the locations to which invasive species may spread (Morisette et al. 2006, Peknicova and Berchova-Bimova 2016). Accurate spatial predictions of invasive spread inform management by identifying priority species (Sheppard et al. 2016) as well as guiding containment boundaries and control strategies (Morisette et al. 2006).

Large scale limitations of species distributions are often attributed to climate (Wisz et al. 2013). However, the rate of spread of an invasive plant species may also vary by taxon (Gallardo et al. 2015) or by non-climatic characteristics of the recipient environment (Timmins and Williams 1991). Factors such as soil-type or species interactions vary in space and can influence invasive species distributions (Buckley et al. 2010, Ibáñez et al. 2014, Mainali et al. 2015). For example, invasion by Pineaceae in Argentina are hindered by a lack of mycorrhizal mutualists (Nuñez et al. 2009). Further, Rouget and Richardson (2003) found that canopy cover of three invasive tree species in South Africa was better predicted by propagule pressure than any environmental factor considered. Critically, human activities intentionally or inadvertently increase invasive propagule pressure (Simberloff 2009). Human activities additionally disturb landscapes, thereby providing favourable conditions for opportunistic species (Timmins and Williams 1991). In fact, invasive species are more common in human disturbed environments and factors such as proximity to roads are often more important predictors of the presence of invasive plant species than climatic variables or vegetative cover (Joly et al. 2011, Meunier and Lavoie 2012, Akasaka et al. 2015, Peknicova and Berchova-Bimova 2016). Despite significant potential for anthropic facilitation of exotic species, this effect is not routinely included in 
species invasion risk assessments (Gallardo et al. 2015, Chapman et al. 2016, Horvitz et al. 2017).

A contrasting pattern of Calluna vulgaris (L.) Hull (Ericaceae) invasion on the North and South Islands of New Zealand provides a unique opportunity to explore how a variety of characteristics of a recipient environment contribute to invasive plant species expansions. $C$. vulgaris has been present on both islands for over 100 years (Chapman and Bannister 1990, 1994, Keesing 1995), but shows remarkably different invasion success. On the South Island, it persists only in small and isolated populations but is widespread and continues to expand its distribution on the North Island (Chapman and Bannister 1994). C. vulgaris control differs between islands with manual herbicide application used on the South Island, particularly at Wilderness Scientific Reserve (Alistair Hay, pers. comm., 2016), and a biocontrol agent, the heather beetle (Lochmaea suturalis), used on the North Island. While both management approaches reduce $C$. vulgaris biomass locally, there has been no success in eradicating the species on either island (Syrett et al. 2000, Evans 2019). In areas where C. vulgaris is abundant, it leads to the decline of endemic plant species and alters insect assemblages (Chapman and Bannister 1990, Keesing 1995, Rogers and Leathwick 1996).

One substantial difference between the two islands of New Zealand that may explain inter-island discrepancies in the invasive success of $C$. vulgaris is the extent and intensity of anthropic pressures. The North Island accounts for $77 \%$ of New Zealand's human population (Statistics New Zealand 2017) and 77.1\% of its GDP (MacPherson 2017). These patterns are reflected in land use trends across the two islands. In 2012, the percentage of land occupied by settlements was 2.9 times higher on the North Island than the South Island (Newsome et al. 2012). Similarly, high intensity land use is more prevalent on the North Island. For example, the North Island has almost twice as much highly productive grassland as the South Island (30\% and $17 \%$ of total land area respectively) and three times as much forestry ( $12.9 \%$ and $4.2 \%$ respectively; Newsome et al. 2012). Conversely, the South Island has more protected areas: nine national parks are on the South Island, while only three are on the North Island (Molloy 2015). 
C. vulgaris has several traits that should enable it to take advantage of climatically and edaphically suitable areas throughout the country and quickly expand its range. Firstly, $C$. vulgaris can achieve very high fecundities $\left(10^{6}\right.$ seeds $\mathrm{m}^{-2} \mathrm{yr}^{-1}$; Miller and Cummins 2001) and its small seed size (Soons and Bullock 2008), together with multiple dispersal pathways (Kleyer et al. 2008), may facilitate rapid spread. For example, of 1334 species recorded as spreading in response to climate change in the Italian Alps, C. vulgaris was in the top $1.6 \%$, achieving rates of spread of $75 \mathrm{~m} \mathrm{yr}^{-1}$ (Dainese et al. 2017). Secondly, C. vulgaris is a generalist with regard to its mutualistic interactions. It forms facultative ericoid mycorrhizas with generalist fungal partners (Bannister and Norton 1974, Johansson 2001). Ericoid mycorrhizal fungal partners are broadly similar worldwide and experimental work indicates that Australian species are compatible with North Hemisphere Ericaceae (Orlovich and Cairney 2004). Furthermore, $C$. vulgaris is both wind and insect pollinated and is a generalist in its pollinator interactions (Descamps et al. 2015); the pollinating fauna in New Zealand is dominated by generalists (Primack 1983). Finally, although C. vulgaris prefers acidic soils, its edaphic requirements are broad relative to many plant species (Walker et al. 2007, Fühner and Runge 2009).

Here, we explored if the discrepancy in $C$. vulgaris invasion success between the North and South Islands of New Zealand can be explained by environmental filtering, or whether it is attributable to differences in anthropic pressure. We used ensemble Species Distribution Models (SDM) to predict the potential distribution of $C$. vulgaris based on the species' occurrence in its native and invasive ranges (see: Jimenez-Valverde et al. 2011) . We mapped other environmental factors that we speculated would be important for limiting the species. Finally, we quantify human disturbance of the landscape across New Zealand. Firstly, we test the hypothesis $\left(\mathbf{H y p}_{\mathbf{1}}\right)$ that neither environmental suitability (climate, soil $\left.\mathbf{p H}\right)$, nor a lack of suitable vegetative communities and mycorrhizal mutualists, limits $C$. vulgaris at large scales on the South Island. We then test the hypothesis $\left(\mathbf{H y p}_{\mathbf{2}} \mathbf{)}\right.$ that $\mathbf{C}$. vulgaris populations are significantly associated with a comparable intensity of human disturbance on the two islands despite the islands differing significantly in human impact conditions. 


\section{Methods}

\section{Climate envelope modelling}

To test $\mathbf{H y p}_{\mathbf{1}}$, that climatic conditions do not determine the variable success of $\mathbf{C}$. vulgaris on the North and South Islands of New Zealand, we built an ensemble SDM. All modelling was done in $\mathrm{R}$ version 3.3.1 ( $\mathrm{R}$ Development Core Team 2016). Bioclimatic variables were downloaded as raster layers at a 2.5 arc-minute resolution (BIOCLIM; retrieved from worldclim.org/bioclim in 2017). These variables are derived from monthly temperature and rainfall values and represent annual trends, seasonality, and environmental extremes. Climatic conditions are based on interpolations of observed climatic data from 1960-1990 (Hijmans et al. 2005). We extracted occurrences ( $n=339,181$ ) for Calluna vulgaris (L.) Hull from the Global Biodiversity Information Facility (GBIF; gbif.org), an open database of species' occurrence data collected from a wide variety of sources. Additional occurrences $(n=3435)$ were provided by Botanical Society of Britain and Ireland (BSBI; bsbi.org). A strong sampling bias was evident across the native range of $C$. vulgaris whereby Western Europe had significantly more occurrence records than Eastern Europe and the margin of densely surveyed areas was clearly delineated by political borders. Sampling bias in geographical space can translate to predictive error if it represents bias in environmental space (Bystriakova et al. 2012). Consequently, the political borders of Denmark, Norway, Sweden, Iceland, Germany, France, Belgium, the Netherlands, Andorra, Spain and Portugal were used to geographically limit the calibration of the model in its native range. Additionally, occurrences in the invasive range in New Zealand were used to calibrate the model (see: Broennimann and Guisan 2008). We removed duplicate records and records that were clearly geographically erroneous. We limited the dataset to one occurrence record per 2.5 arcminute grid cell to partially correct for remaining observer bias (Dormann et al. 2012). The number of presences in the final model was 44,412 . Pseudoabsences were drawn exclusively from the native range of $C$. vulgaris in Western Europe as the species is unlikely to have reached climatic equilibrium in New Zealand. We selected environmentally stratified pseudo-absences, because the dataset included a large number of presences, sampling bias remained evident within Western Europe and because environmental stratification promotes sensitivity over specificity of models (Barbet-Massin et al. 2012). 
Sensitivity is favourable for invasive species' distribution models as the precautionary principle suggests that prioritization of the precise inclusion of all possible invasion prone habitats (Jimenez-Valverde et al. 2011). Ten pseudo-absence replicates, each comprising of 4441 pseudo-absences (resulting in a presence: absence ratio of 10:1; see Barbet-Massin et al. 2012), were created using the 'SRE' option in the data formatting function in the 'biomod2' package, version 3.3.7 (Thuiller et al. 2009), with a quantile of 0.025 , which corresponds to a $95 \%$ confidence interval in normal distributions. We created another, geographically independent testing dataset, as independent data are a powerful test of model performance (Broennimann and Guisan 2008). The testing dataset ( $n=502)$ spanned occurrences in Eastern Europe, Siberia, Australia and North America. The testing dataset was corrected for observer bias and error as described above for the training dataset and excluded points within $50 \mathrm{~km}$ of the geographic boundary of the training dataset.

To identify important climatic predictors of $C$. vulgaris, we calibrated preliminary climatic niche models using climatic variables (7 of 19) chosen a priori that are considered ecologically significant for $C$. vulgaris (listed in Appendix 5). In order to include the largest possible variability in model predictions, we applied Generalized Linear Model (GLM; McCullagh and Nelder 1989) , Classification Tree Analysis (CTA; Breiman 2001), Artificial Neural Network (ANN; Ripley 1996), Surface Range Envelope (BIOCLIM/SRE; Busby 1991) and Random Forest (RF; Breiman 2001) algorithms. We calibrated these preliminary climatic suitability models across five pseudoabsence datasets. Variable importance was calculated within the 'BIOMOD_Modeling' function for each modelling algorithm with three permutations of each variable. We discarded climatic variables that had the lowest average importance.

We calibrated the final model using four climatic data layers: annual mean temperature, maximum temperature of the warmest month, minimum temperature of the coldest month and annual precipitation. The same selection of algorithms was calibrated 3 times on a random $80: 20$ split of the data using each of 10 presence-pseudoabsence datasets; this resulted in 150 individual models. A True Skill Statistic (TSS) and a Relative Operating Characteristic (ROC) value were calculated for each model. Visual inspection of predictions in the native range indicated no apparent geographical bias in model prediction error. Additionally, we assessed the 
sensitivity of models on the geographically independent testing data. Ensemble models, which synthesize multiple initial conditions and algorithms, tend to yield lower error rates than any of the individual forecasts of which they are composed and are considered more robust when used to model novel climates and/or geographic regions (Araújo and New 2007, Thuiller et al. 2009). We built an ensemble model from individual models that had a TSS greater than 0.8 and predicted $>95 \%$ of occurrences in the testing dataset. Models which satisfied these validation criteria and were combined by mean $(n=146)$. Climatic suitability from the model ensemble was projected across New Zealand together with a coefficient of variation of individual model predictions. A Multivariate Environmental Similarity Surface (MESS) was generated in R using the 'dismo' package v 1.1.4 (Hijmans et al. 2017). MESS maps indicate, in a geographically explicit manner, similarities between training range and projection range climates: where, and to what extent, a model is extrapolating beyond its training dataset or predicting in conditions which are poorly represented in the training dataset (Elith et al. 2010).

\section{Non-climatic environmental filters}

We further tested $\mathbf{H y p}_{1}$, that non-climatic filters - soil $\mathrm{pH}$, suitable vegetative communities or mycorrhizal symbiont presence - do not limit C. vulgaris invasion at large scales in New Zealand. We downloaded the New Zealand Fundamental Soil Layer soil pH (Landcare Research NZ Ltd) from the Land Resource Information Systems portal (LRIS; Iris.scinfo.org.nz, accessed June 2017). The data are part of the New Zealand Land Resource Inventory (NZLRI), which delineates physiographic polygons at a 1: 50,000 scale and interpolates soil pH values based on 1500 soil cores distributed across the country. Soil pH requirements of $C$. vulgaris are well defined - C. vulgaris prefers acidic soils (pH 3.4 - 6.5; Walker et al. 2007) but can occur on slightly basic soils (<7.1; Fühner and Runge 2009). In our model, soils with a pH $3.4-6.5$ were considered suitable for $C$. vulgaris, while pH 6.5 - 7.1 was deemed marginally suitable. We mapped vegetative suitability using the Vegetative Map of New Zealand (Landcare Research NZ Ltd) which we downloaded from the LRIS portal. This resource compiles a variety of vegetative cover dataset and ascribes one of 47 vegetative classes to polygons with a geographic precision of $250 \mathrm{~m}$. We considered 36 of the 47 cover classes as potential habitat for $C$. vulgaris (see Appendix 5). Areas considered inappropriate included all forests with closed canopies and areas 
with permanent ice cover. Caution is warranted in interpretation of this data layer as the data were collected in 1987. To map mycorrhizal symbiont presence, we mapped occurrences of other plant species known to host ericoid mycorrhizal fungi which are morphologically similar to those of $C$. vulgaris. Several New Zealand native plant species within the Ericaceae and Epacridaceae families form such associations (McNabb 1961). All occurrence data for these species in New Zealand were downloaded from GBIF (20 species, listed in Appendix 5; $n=$ 18,254).

\section{Human impact}

Next, we tested $\mathbf{H y p}_{\mathbf{2}}$ that $C$. vulgaris populations are significantly associated with a comparable intensity of human disturbance on the two islands despite the islands differing significantly in human impact conditions. Gallardo et al. (2015) modelled the influence of a variety of spatial variables on the distribution of 17 invasive terrestrial plants in Europe. The Global Human Influence Index (HII), distance to roads and population density were determinative of the invasive spread of terrestrial plants (Gallardo et al. 2015). We used the mean response curves of invasive terrestrial plants to three variables (HII, Euclidean distance to road and population density), as presented by Gallardo et al. (2015) to parameterise a "human footprint" map. Spatially explicit HII was downloaded at a $1 \mathrm{~km}^{2}$ resolution (retrieved from sedac.ciesin.columbia.edu, July 2017). HII is a measure of human disturbance, rating landscapes from 0 (near pristine) to 64 (very degraded) based on human population pressure, urbanization and other land use, and human access, via roads, navigable rivers and several other factors. In New Zealand, HII varies between 0 and 60 . We extracted the values of $\mathrm{HII}$ to a raster layer at a 2.5 arc-minute resolution. We created a raster layer with a 2.5 arc-minute resolution and calculated the proximity of the raster cell centre to the closest road in ArcMap version 10.4.1 (ESRI 2011) based on the "NZ Road Centrelines" shapefile (1:50, 000; downloaded from data.linz.govt.nz, July 2017). Proximity to roads was calculated by Euclidean distance. Population density (residents $/ \mathrm{km}^{2}$ ), based on 2013 census data, was extracted from the "New Zealand population density by meshblock" shapefile (downloaded from koordinates.com, July 2017) to a 2.5 arc-minute resolution raster. Correlations between these layers were tested for in $\mathrm{R}$ and were low (Pearson's $r^{2}<|0.5|$ ). The mean relationship of the 17 species to these three 
factors, as presented by Gallardo et al. (2015) was used to calibrate the "human footprint" raster layer. The three suitability scores were weighted according to the average contribution of each variable to invasive species occurrence as presented by Gallardo et al. (2015), and summed to create a "human footprint" map.

We compared the intensity and geographic extent of human impact of the North and South Islands by extracting the "human footprint" values for all raster cells and comparing the distribution of raster cell values of the North and South Islands. "Human footprint" raster cell values were non-normally distributed (Bartlett's $\mathrm{K}^{2}=665.75, \mathrm{df}=1, \mathrm{p}<0.001$ ), with a bimodal distribution on the South Island. We therefore used the "shuffle" function in the package “permute" v. 0.9.4 (Simpson 2016), a non-parametric test (Matchett et al. 2015). In this test, we compared the observed mean difference of human footprint raster cell values between the two islands to a null distribution calculated from 5000 permutations. Next, we tested whether $C$. vulgaris populations were associated with different mean "human footprint" values on the two islands. To do this, we compared the observed mean difference between human footprint values at $C$. vulgaris occurrences on the North and South Islands with a null distribution based on 5000 permutations, also using the "shuffle" function. 


\section{Results}

\section{Climate envelope modelling}

All algorithms performed well according to TSS and ROC evaluations, with average performance at 0.85 and 0.95 , respectively. Performance of individual algorithms is shown in Table 5.1. When tested on geographically independent occurrences outside of the model training range, the average sensitivity (correct prediction of true presences) of the models was $96.68 \%$. Four ANN models had $<95 \%$ sensitivity and were therefore excluded from the ensemble model. The ensemble model had a TSS of 0.872 . We predict high climatic suitability for $C$. vulgaris across large areas of both islands (Figure 5.1A). On the North Island, high climatic suitability was found on the lowlands of the Wellington, Manawatu-Wanganui, Gisborne, Waikato and Hawkes Bay regions. Lower climatic suitability was predicted for Taranaki and Northland, as well as montane areas across the rest of the island. On the South Island, regions east of the Southern Alps were considered highly climatically suitable by all models; low suitability was predicted on the West Coast as well as in small areas of Central Otago. Across both Islands, areas where the ensemble model predicted low climatic suitability were associated with high coefficients of variation between individual model predictions (Figure 5.1B). The MESS map suggests few areas where the model extrapolates outside of its training range (indicated in red in Figure 5.1C): two locations on the West Coast of the South Island, and portions of Mt Taranaki and Mt Ruapehu on the North Island. However, many areas of New Zealand are subject to environmental conditions which are represented by limited data in the training dataset (indicated in white in Figure 5.1C). These regions were associated with higher coefficients of variation among models and predictions of lower likelihood of climatic suitability for $C$. vulgaris by the ensemble model. 
Table 5.1: Performance of Calluna vulgaris climatic envelope models. Average and minimum TSS and ROC evaluation statistics, for all models, grouped by modelling algorithm, together with average and minimum sensitivity when tested on geographically independent data.

\begin{tabular}{|c|c|c|c|c|c|c|}
\hline Algorithm & $\begin{array}{l}\text { Average } \\
\text { TSS }\end{array}$ & $\begin{array}{l}\text { Minimum } \\
\text { TSS }\end{array}$ & $\begin{array}{l}\text { Average } \\
\text { ROC }\end{array}$ & $\begin{array}{l}\text { Minimum } \\
\text { ROC }\end{array}$ & $\begin{array}{l}\text { Average } \\
\text { Sensitivity }\end{array}$ & $\begin{array}{l}\text { Minimum } \\
\text { Sensitivity }\end{array}$ \\
\hline $\begin{array}{r}\text { Artificial Neural } \\
\text { Network }\end{array}$ & 0.809 & 0.742 & 0.951 & 0.929 & 93.96 & 1.59 \\
\hline $\begin{array}{r}\text { Classification Tree } \\
\text { Analysis }\end{array}$ & 0.865 & 0.857 & 0.953 & 0.945 & 99.61 & 99.60 \\
\hline $\begin{array}{r}\text { Generalized Linear } \\
\text { Model }\end{array}$ & 0.824 & 0.801 & 0.955 & 0.951 & 99.06 & 99.00 \\
\hline Random Forest & 0.867 & 0.855 & 0.971 & 0.967 & 98.22 & 97.01 \\
\hline $\begin{array}{r}\text { Surface Range } \\
\text { Envelope }\end{array}$ & 0.869 & 0.860 & 0.934 & 0.930 & 92.57 & 92.43 \\
\hline
\end{tabular}




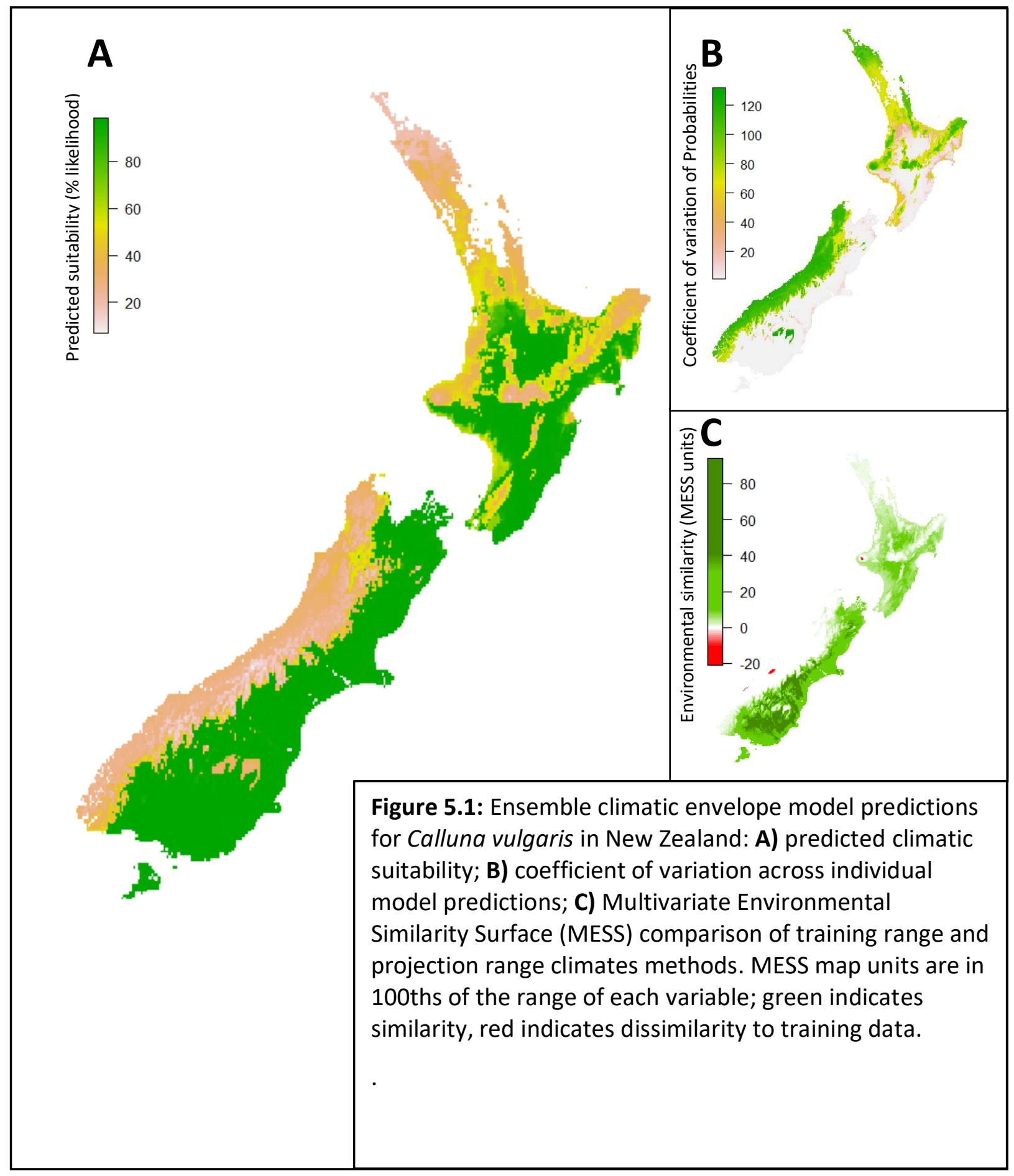




\section{Non-climatic environmental filters}

We found no evidence for limitation of $C$. vulgaris in New Zealand by non-climatic environmental constraints. The majority of New Zealand has soil pH within an appropriate range for $C$. vulgaris (Figure 5.2). Small areas within Otago and the Waikato are too alkaline (pH >7.1). Habitats with marginal pH appear throughout New Zealand but are neither prevalent nor continuous. Areas in high alpine zones and Stewart Island are missing data and therefore could not be assessed for edaphic suitability. Large areas of New Zealand also host vegetative communities at risk of invasion by $C$. vulgaris (Figure 5.3). Areas with vegetative communities which are not suitable for $C$. vulgaris appear in patches throughout New Zealand but form contiguous regions on the West Coast of the South Island, as well as Hawke's Bay, the Waikato and Manawatu-Wanganui on the North Island. Likewise, native plant species that host ericoid mycorrhizal fungi which are known to form symbioses with $C$. vulgaris are distributed throughout New Zealand (Figure 5.4), but occurrence records are more frequent in montane regions throughout the country. Host plants are scarcer but not absent in Southland, Otago and lowland Canterbury on the South Island, and lowland regions in Wellington, Hawke's Bay, Manawatu-Wanganui, Taranaki and Gisborne on the North Island. 


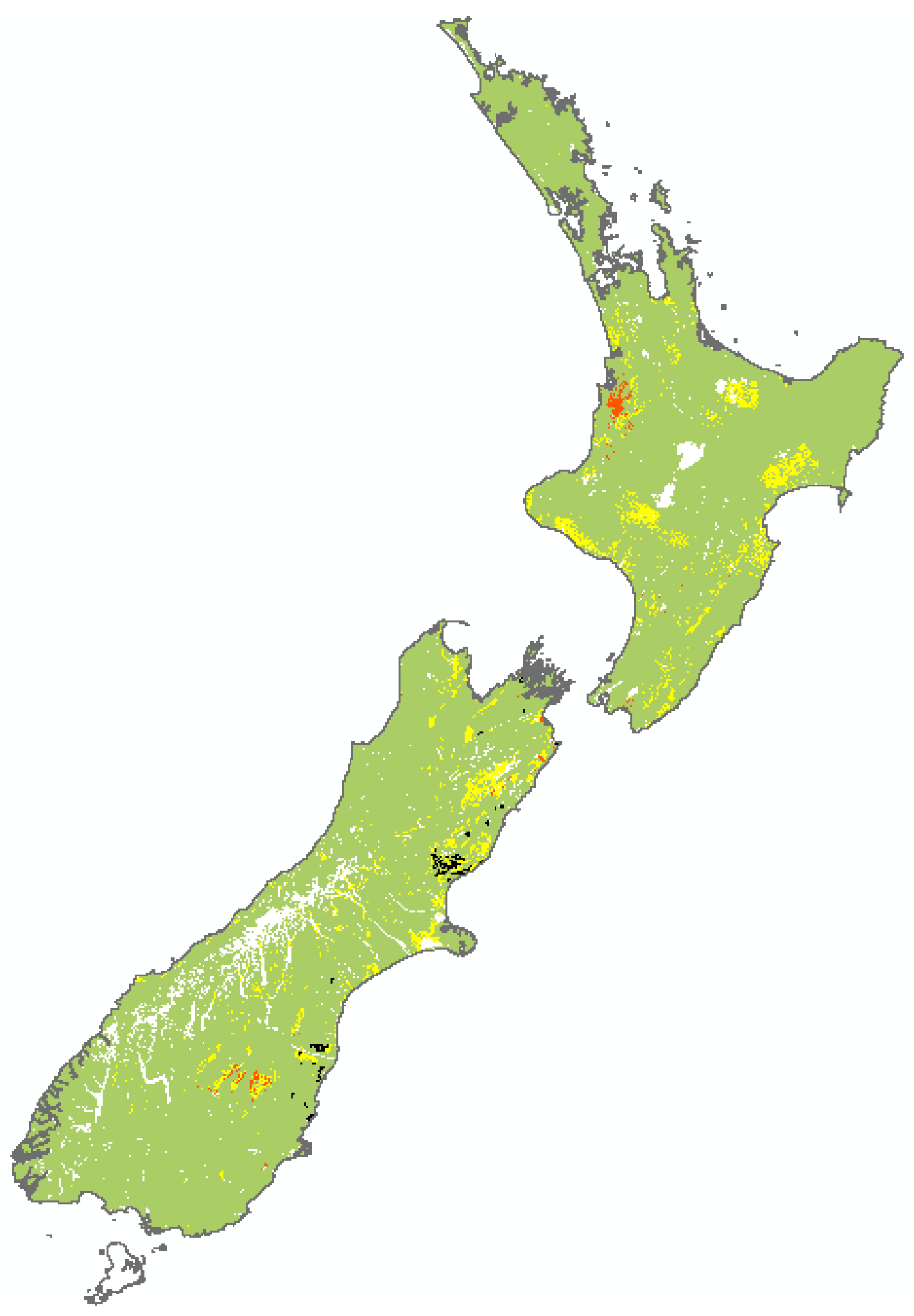

Figure 5.2: Soil pH suitability for Calluna vulgaris across New Zealand; soils considered highly suitable $(\mathrm{pH}=3.4-6.5)$ are indicated in green, marginal soils in yellow $(\mathrm{pH}=6.5-7.1)$, and unsuitable in red $(\mathrm{pH}>7.1)$. Missing data are in white. 


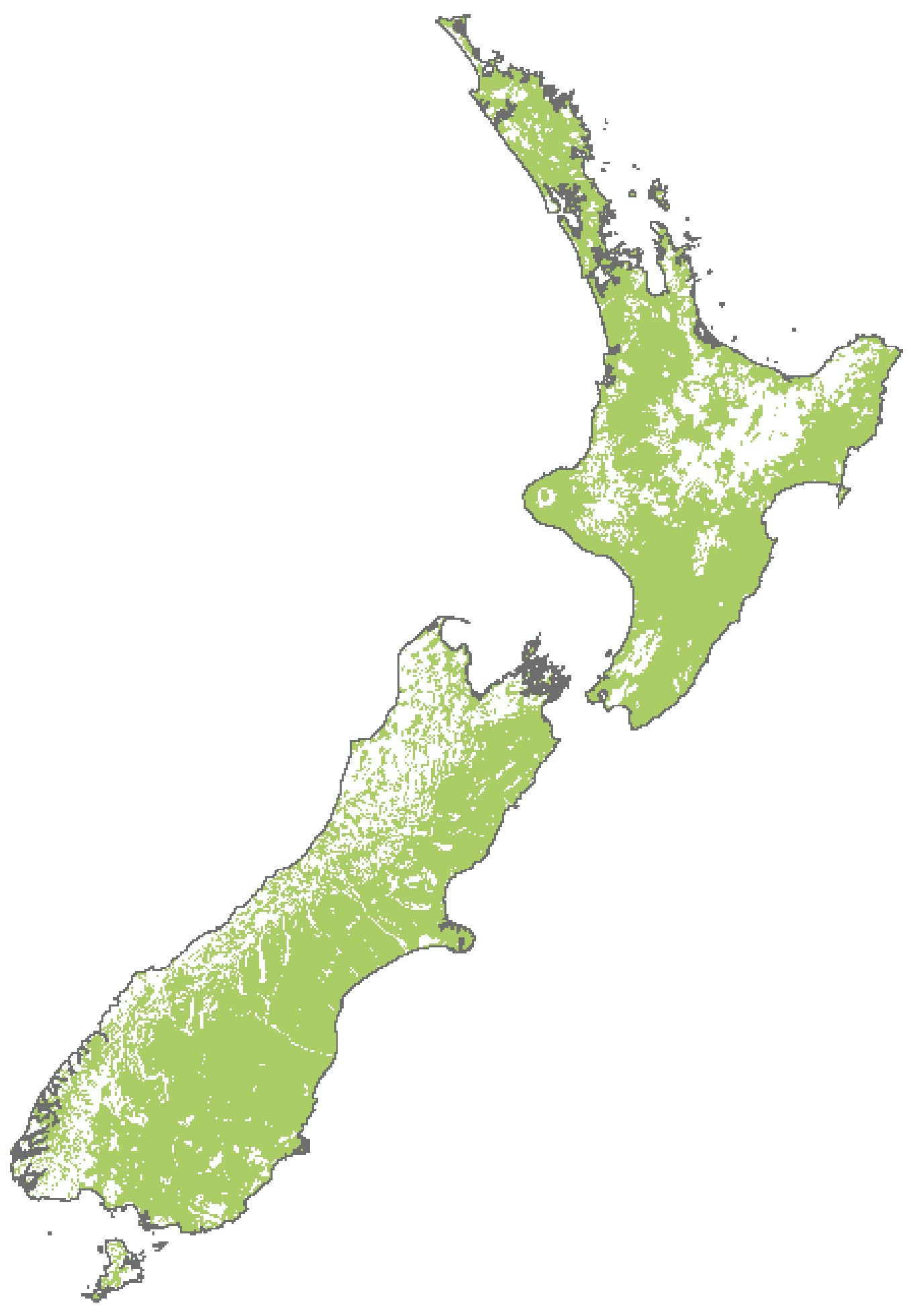

Figure 5.3: Vegetative community suitability for Calluna vulgaris across New Zealand. Vegetative communities deemed at risk of incursion by $C$. vulgaris across New Zealand are indicated in green and those not considered at risk in white; community types considered at risk of incursion are listed in Appendix 5. 


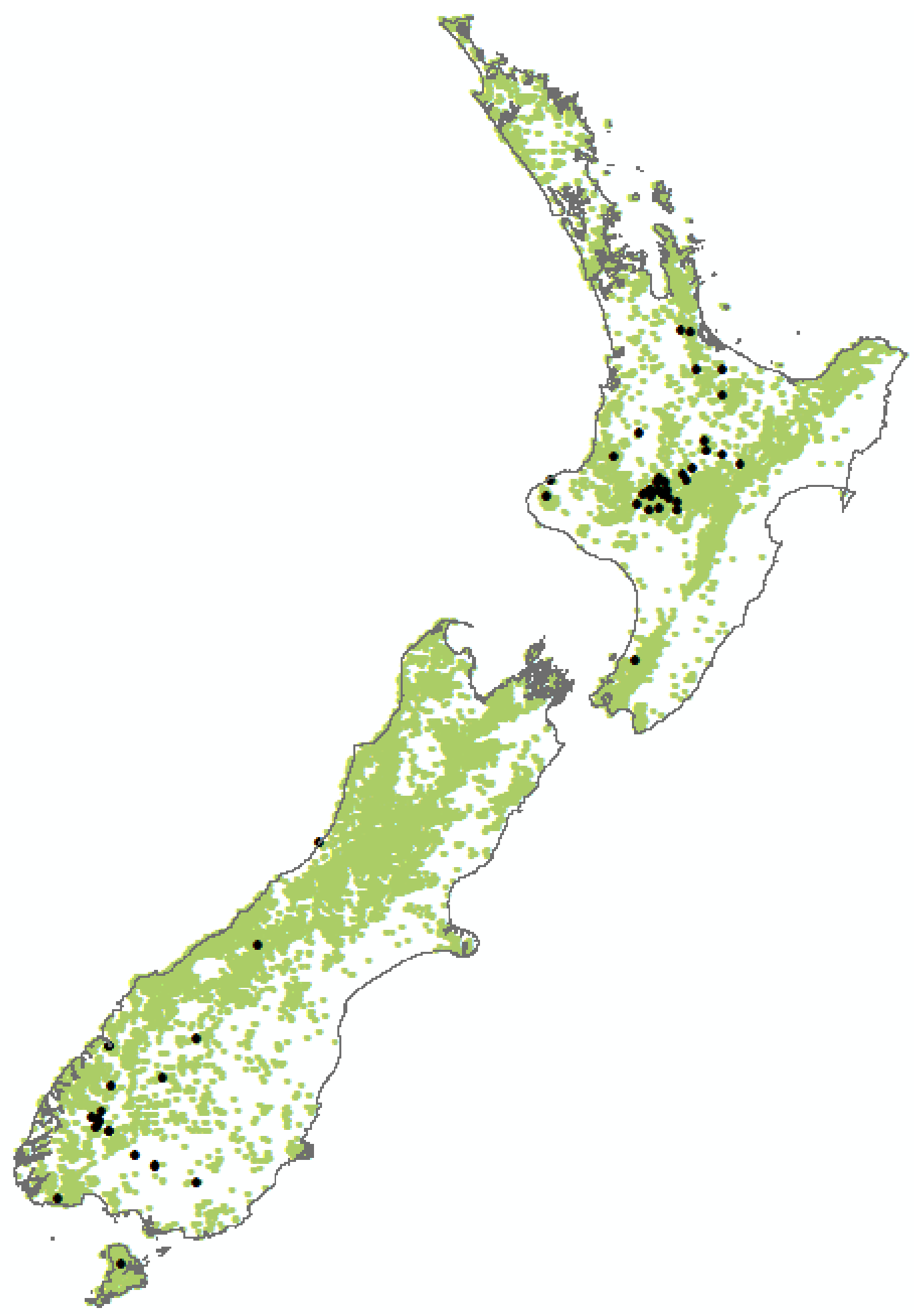

Figure 5.4: Mycorrhizal fungal partner presence for Calluna vulgaris across New Zealand. Occurrences of native ericaceous plant species known to host ericoid mycorrhizal fungi are shown in green, together with known occurrences of $C$. vulgaris (black). 


\section{Human impact}

Hyp $_{2}$ was that $C$. vulgaris populations are significantly associated with human disturbance on the two islands despite the islands differing significantly in human impact conditions. Our novel metric, "human footprint", varied from 0.001 (low human impact) to 0.161 (high human impact) in New Zealand. A histogram of "human footprint" raster cell values for each island is displayed in Figure 5.5A. Mean "human footprint" was higher on the North Island (mean "human footprint" $=0.099$ ) than the South Island (mean "human footprint" $=0.071$ ), which constituted a significantly larger mean difference than expected based on 5000 permutations ( $p$ $=0.02$ ). Only 118 of 5000 permutations resulted in a smaller mean difference than the observed value. While the islands have a similar number of raster cells with high "human footprint" values, the South Island has a bimodal distribution as it includes large areas with very low levels of "human footprint". Conversely, mean difference of "human footprint" at C. vulgaris occurrences was not significantly different between the islands (Figure 5.5B; $p=0.188$ ). Mean "human footprint" on the North Island at C. vulgaris occurrences was 0.103 and on the South Island was 0.097 . Most $C$. vulgaris occurrences on both islands were within raster cells with medium to high levels of "human footprint" and very few instances recorded in cells with low values (Figure 5.6). On the North Island the distribution was centred around the central volcanic plateau (where the species was introduced); remaining populations are distributed in high and medium "human footprint" conditions. In contrast, "human footprint" on the South Island is more fragmented. Occurrences of $C$. vulgaris are scattered throughout the south and west of the island but are all clustered around areas of relatively higher "human footprint". 


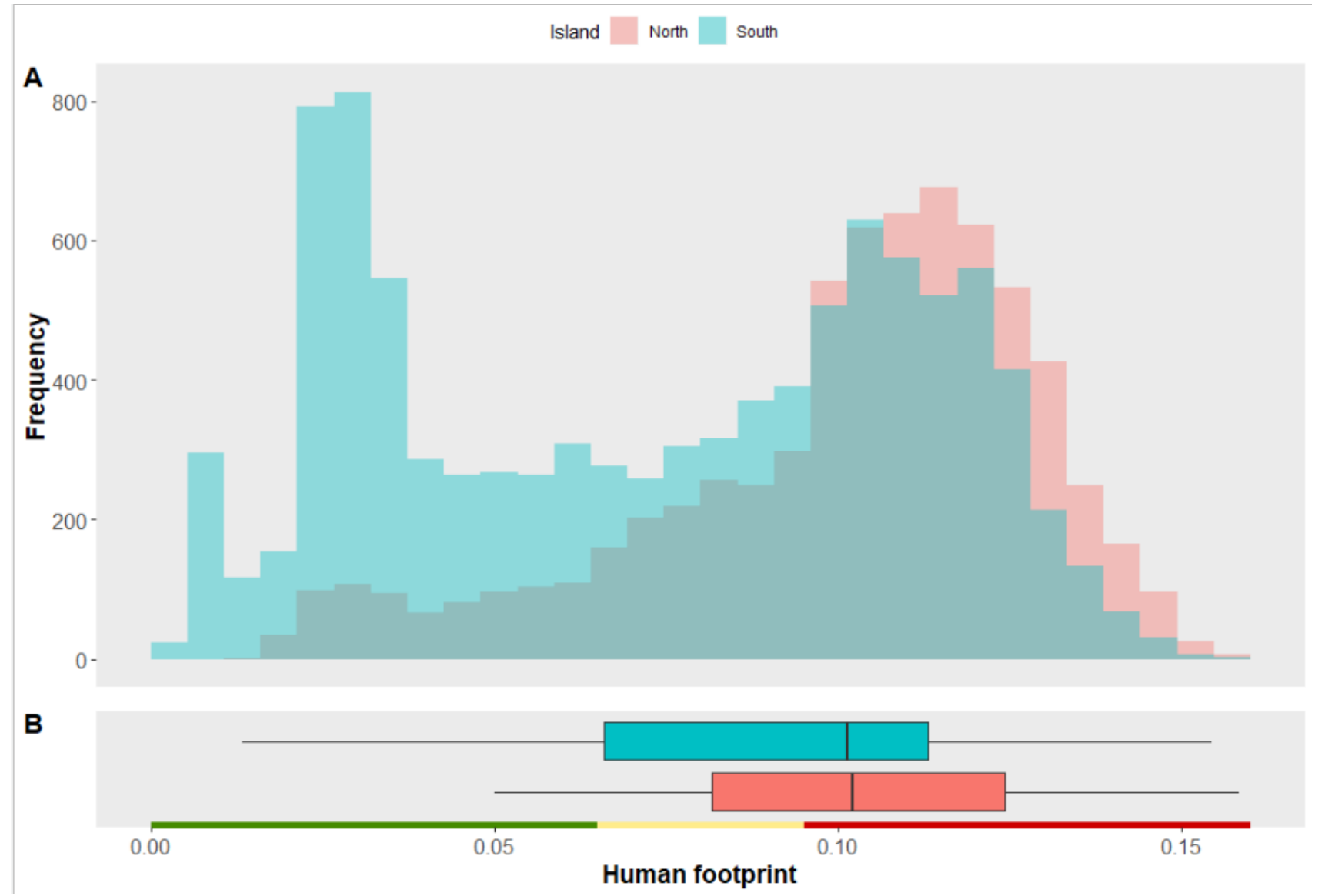

Figure 5.5: 'Human footprint' values: A) across the North and South Islands of New Zealand; B) at Calluna vulgaris occurrences on each island. North and South Islands of New Zealand are displayed in red and blue, respectively. Histogram indicates the proportional frequency of raster cells containing each value across New Zealand. Box and whisker plot indicates the range of 'human footprint' values at $C$. vulgaris occurrences. Colour bar indicates approximate colour mapping in Figure 5.6, and corresponds to referenced levels in the text: "low" (green), "medium" (yellow) and "high" (red). 
- Calluna vulgaris populations
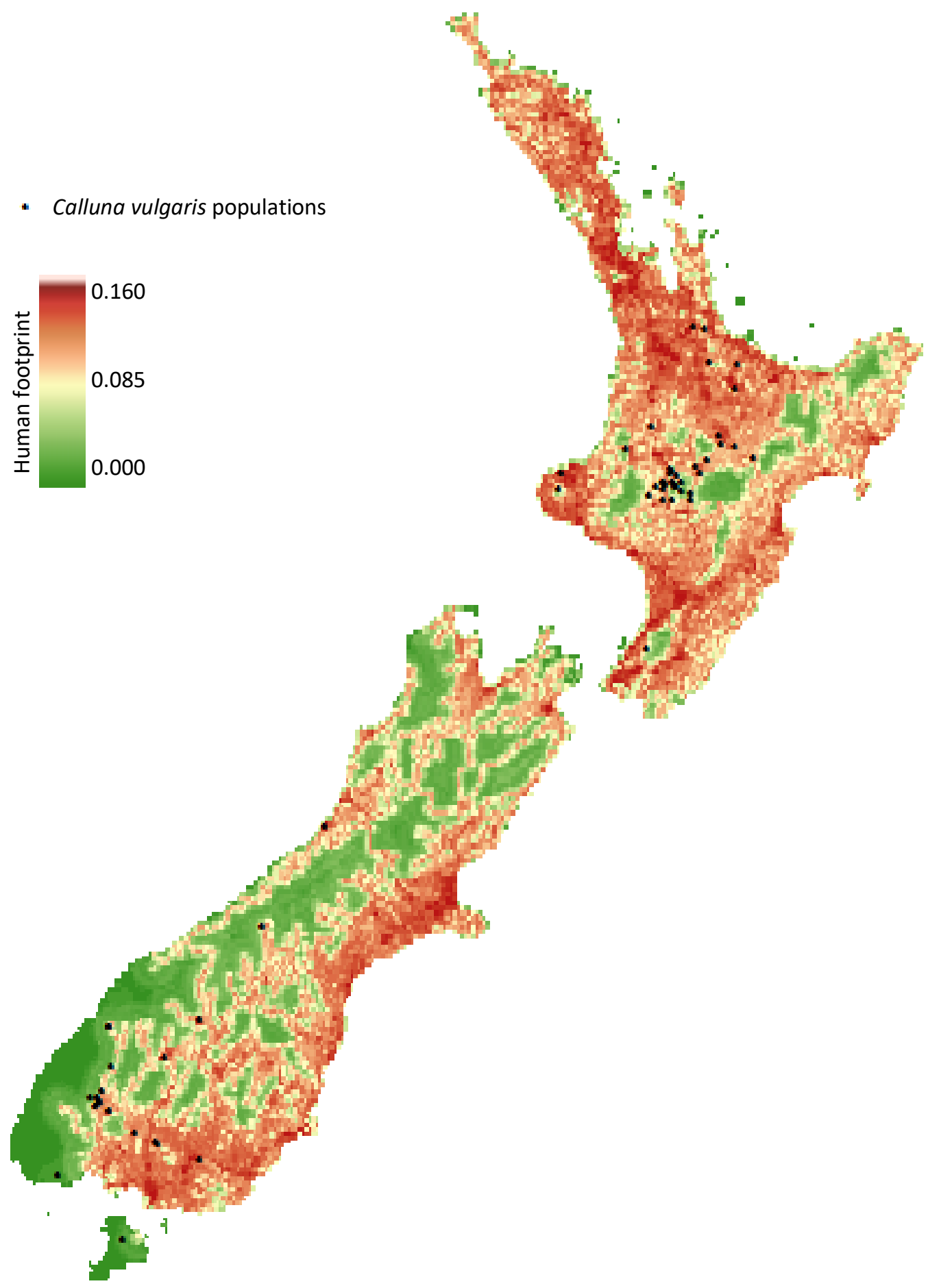

Figure 5.6: Human disturbance intensity across New Zealand. 'Human footprint' is a metric comprised of three factors- distance to the nearest road, Human Impact Index and human population density. High 'human footprint' (approximately $>0.095$ ) is indicated in red and low 'human footprint' (approximately $<0.065$ ) in green $(\mu=0.085$ across New Zealand). Black points indicate known occurrences of Calluna vulgaris. 


\section{Discussion}

Invasive species become increasingly difficult to manage once they establish in their novel range. Identifying habitats at risk of invasion is therefore a critical goal for invasion modelling studies. Here, we modelled $C$. vulgaris - an invasive plant on the North Island of New Zealand which is present, but not widely problematic, on the South Island - to understand the role of environmental filters and human disturbance in invasive. We find support for both of our hypotheses. We find that environmental suitability (climatic, soil, mycorrhizal, or plant community) does not constrain the large-scale distribution of $C$. vulgaris on either the North or South Islands of New Zealand $\left(\mathbf{H y p}_{\mathbf{1}}\right)$. C. vulgaris occurrences are significantly correlated with both the intensity and spatial patterns of human disturbance, suggesting a role for anthropic facilitation in the spread of this species $\left(\mathbf{H y p}_{\mathbf{2}}\right)$. This suggests that $\mathbf{C}$. vulgaris may become a problematic invasive species on the South Island in the future as disturbance intensifies. Increasing anthropogenic pressures on the South Island may promote movement of $C$. vulgaris into more suitable localities or may interact with genetic processes to facilitate an escape of populations from the lag phase of establishment in the future. Our study illustrates the utility of SDM in identifying the mechanisms constraining or promoting the spread of invasive plant species.

Geographic projections of environmental suitability provide preliminary, but reliable hypotheses of sites at risk of invasion (Milbau et al. 2009, Jimenez-Valverde et al. 2011). Our study contributes to understanding of the invasive potential of $C$. vulgaris in New Zealand by illustrating that environmental filtering does not preclude its invasion across the country. While Chapman and Bannister (1994) reported broadly similar climatic conditions at their study sites in New Zealand to the native range of $C$. vulgaris, our climate envelope model illustrates the extent to which New Zealand is climatically analogous to its native distribution. Our models suggest that small portions of New Zealand are climatically unsuitable for $C$. vulgaris. However, low climate suitability predictions are universally associated with a high coefficient of variation in our predictions, which is driven by dissimilarity of the local climate to climates represented in the training dataset (as indicated by the MESS map). Consequently, it is difficult to assess the climatic suitability in these regions and precautionary principle suggests that vigilance regarding 106 
C. vulgaris spread remains wise in these regions. Similarly, soil chemical and biotic characteristics are unlikely to limit the invasion of $C$. vulgaris in New Zealand at large spatial scales on either island. Soil pH across New Zealand is predominantly within the ranges of 3 6.5, which is highly suitable for C. vulgaris (Walker et al. 2007, Fühner and Runge 2009). Likewise, we found a wide distribution of ericaceous plants capable of hosting mycorrhizal symbionts throughout New Zealand and near South Island C. vulgaris populations. This suggests a low likelihood that mycorrhizal symbiont absence is a mechanism limiting $C$. vulgaris spread at large spatial scales. Further, our vegetative cover map indicated that plant communities susceptible to invasion by $C$. vulgaris - such as native herbfields, tussock grasslands and bogs (Chapman and Bannister 1990, Rogers and Leathwick 1996) - are common throughout New Zealand, and therefore habitat-limitation is unlikely to be a mechanism limiting the spread of $C$. vulgaris. Indeed, the local success of $C$. vulgaris populations on the South Island in areas such as the Wilderness Scientific Reserve and Ben Callum peat bog, support the conclusion that the environment is suitable. Despite a lack of large-scale limitation, these biotic and abiotic factors may nevertheless contribute to a lag between the establishment and invasive expansion of $C$. vulgaris. This is because invasion involves the arrival of propagules to a spatially heterogenous habitat and local conditions at the site of arrival strongly predict establishment success by determining the establishment, growth and the sexual reproductive output of individuals (Schreiber and Lloyd-Smith 2009).

Local population growth precedes range expansion of invasive species (Theoharides and Dukes 2007). Therefore, local heterogeneity of environmental filters may constrain or enhance invasive spread because local conditions are critically linked with the demography of populations, and therefore with their potential for exponential growth (Schreiber and LloydSmith 2009). For example, decreased vegetative and sexual performance of $C$. vulgaris is associated with suboptimal soil nutrient or moisture regimes (Chapman and Bannister 1994, Walker et al. 2007). Soil characteristics are notoriously variable at small spatial scales (Gurevitch et al. 2011) and inadequate soil at small scales may contribute to patterns of invasion (Schreiber and Lloyd-Smith 2009). Rogers and Leathwick (1996) related the invasive spread rates of $C$. vulgaris in Tongariro National Park (North Island) to topographic position and 
hypothesised that invasive spread of isolated populations was limited by organic, waterlogged or acidic soils. Similarly, a lack of mycorrhizal mutualists may decelerate exotic species spread (Nuñez et al. 2009, Bunn et al. 2015) by depressing their reproduction or nutrition (Urcelay et al. 2017). Mycorrhization is associated with higher aboveground biomass in C. vulgaris, particularly in poor edaphic conditions (Bannister and Norton 1974). Therefore, although $C$. vulgaris is not obligately mycorrhizal (Bannister and Norton 1974), mycorrhization could contribute to fitness locally and therefore invasive spread patterns. Ericoid mycorrhizal fungi are widely found in New Zealand soils beneath native communities (McNabb 1961, Orlovich and Cairney 2004), however it is unclear how commonly these symbionts are found in soils in agricultural landscapes. In some regions, particularly those with higher agricultural pressure, occurrence records for ericoid mycorrhizal fungi hosting plants are sparse and it is possible that a lack of mycorrhizal mutualists is a limiting factor at these smaller scales. Conversely, Orlovich and Cairney (2004) proposed mycorrhizal facilitation as an explanation for the rapid spread of C. vulgaris in Tongariro National Park. Finally, the characteristics of the plant communities into which propagules arrive may influence the establishment success of invasive species (Sheppard et al. 2018). For example, high density native communities may be more resistant to invasion. Plant-plant competitive interactions most typically affect the distributions of species at local scales (Powell et al. 2013). However, such small-scale limitations are unlikely to translate into long-term exclusion of this species. Notably, none of these factors- soil pH, mycorrhizal symbiont presence, or vegetative cover- appears to substantially differentiate the suitability of the environmental conditions on the North and South Islands for C. vulgaris.

We found that the two largest islands of New Zealand differ saliently in human impact: larger spatial extents of medium to high human impact are observed on the North Island than on the South Island. Despite this significant difference in human impact, $C$. vulgaris populations are disproportionately found in areas with a medium to high levels of human disturbance on both islands, and we reveal evidence of non-random association of $C$. vulgaris occurrences with spatial patterns human disturbance. This correlation suggests that human disturbance may contribute to the diverging trajectories of $C$. vulgaris invasive spread on the two islands. These findings align with previous work which found human disturbance facilitates the invasive spread 
of introduced plant species (Hulme 2009, Dainese et al. 2017, Horvitz et al. 2017). Notably, we find that very high land use intensity is also not correlated with $C$. vulgaris occurrence, indicating that not all human impact is beneficial to the invasion of this species. For example, the conversion of pastoral land to diary may exclude $C$. vulgaris. Notwithstanding such specific cases, human disturbance is known to contribute to escape from lag phase by increasing rates of rare, highly stochastic, long distance dispersal events to more suitable habitat (Morisette et al. 2006, Pielaat et al. 2006, Nathan 2008). For example, Horvitz et al. (2017) demonstrated that for 17 invasive plant species in China, models incorporating exclusively natural dispersal mechanisms profoundly underestimate the movement of exotic propagules. Other mechanisms by which human disturbance may contribute to the spread of invasive plants is by perturbing ecosystems and creating more suitable habitat for exotics such as C. vulgaris (Chapman and Bannister 1994). We can also expect an interaction between these two processes: anthropic augmentation of patch quality and the connectivity of patches may together facilitate exotic species spread. Finally, human-mediated dispersal of propagules, may interact with genetic processes. Dispersal to both new locations with highly suitable habitat, or between metapopulations may be of particular importance in genetically depauperate and isolated populations (Saltonstall 2002, Simberloff 2009, Bock et al. 2015). Strikingly different introduction efforts were made with $C$. vulgaris on the North and South Islands. On the North Island, multiple large-scale plantings were made using seed sourced from several European source populations, whereas on the South Island the introduction effort has been substantially smaller- for example, the Ben Callum Peat Bog population was established with "just a handful of seeds" (Mark Heslip, pers. comm., 2016). However, the genetic structure of $C$. vulgaris populations in New Zealand has not been studied. We therefore do not know whether the lag phase observed in C. vulgaris on the South Island is related to genetic processes and we recommend that further work is undertaken to clarify whether a genetic bottle-neck to invasive spread exists on the South Island.

In this study, as in many others, the climatic envelope model is limited in its predictive power because climatic conditions in the novel range differ from those represented in the native range of the invasive species. Our model indicates that areas with lower climatic 
suitability for $C$. vulgaris occur in New Zealand. However, these areas are consistently associated with higher coefficients of variation for the ensemble model as well as environmental conditions that are poorly represented in the training dataset. Projections of correlative models outside of the training range lack theoretical justification (Buckley et al. 2010). Conjectural projections are prone to diverging predictions and although the ensemble modelling approach can help us to identify the central tendency, predictions should be treated with caution (Araújo and New 2007, Thuiller et al. 2009, Elith et al. 2010). This is particularly true in the present case: $C$. vulgaris thrives throughout Tongariro National Park and exists in small populations along the West Coast and in Taranaki National Park. These populations directly challenge the low likelihood prediction ( $40 \%$ ) of appropriate climate provided by the model for these regions. There are several possible explanations for poor model predictive performance at populations which inhabit edge-of-training-range climates in this study. Firstly, C. vulgaris is capable of surviving and reproducing in a wider range of conditions than those which are represented in its native range. Secondly, the environmental predictors used insufficiently capture the climatic variability of these locations. For example, the edge-oftraining-range conditions on the West Coast of the South Island are primarily driven by precipitation patterns. However, it is unknown how differences in precipitation translate to differences in soil moisture content (Chapman and Bannister 1994). Finally, C. vulgaris in New Zealand could be occupying a novel niche. This could be the result of novel biological interactions or enemy release (Araújo and Guisan 2006) or the result of rapid evolution in the novel range (Simberloff 2009). Further studies comparing the ecology of this species in its invasive and native ranges could corroborate one or more of these explanations, thereby aiding in the interpretation of such model projections. Our results highlight the importance of measuring the dissimilarity of climates in training and projection ranges of SDMs to correctly interpret model findings. Furthermore, we highlight the contribution of ensemble modelling approaches to elucidating uncertainty in predictions from SDM.

Here we explore the factors limiting the distribution of a plant species that varies in its invasive success between two islands in an exotic range. We found support for our hypothesis that $C$. vulgaris invasion is not limited by environmental suitability, indicating further risk of 
invasive spread of $C$. vulgaris throughout New Zealand. We advocate the use of the species' biology to constrain non-climatic variables such as edaphic and vegetative requirements as well as the consideration of human disturbance of areas of interest. Our study system illustrates that the introduction of an exotic plant species to two islands, both of which have an abundance of climatically, edaphically and vegetatively suitable habitat, but different degrees of anthropogenic disturbance and introduction efforts, can yield strikingly divergent invasion outcomes a century later. We conclude that whilst these environmental filters may have an impact at local scales, these limitations may be overcome by rare, long-distance dispersal events. This is an important finding in a context of increasingly frequent, human-mediated longdistance dispersal events. 
6 Conclusions

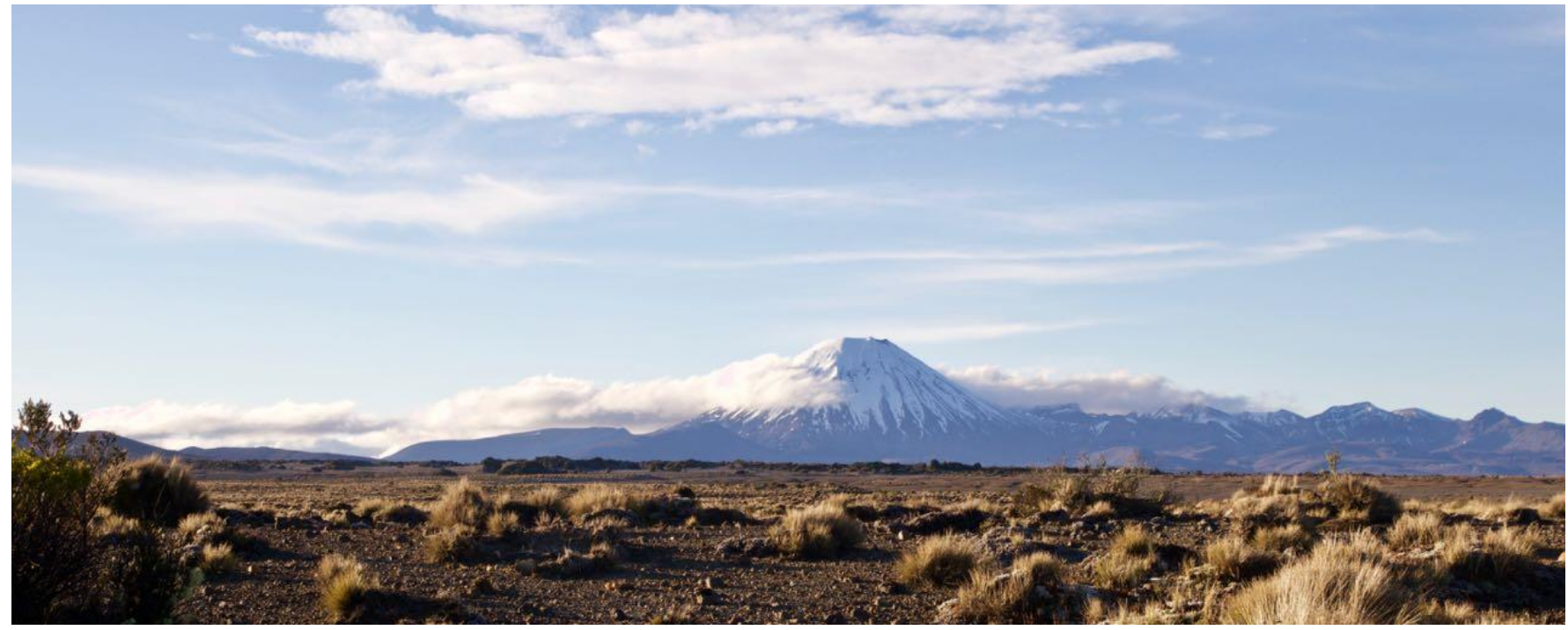

Mount Ngauruhoe and the Rangipo desert at sunset.

Wim Coosemans, 2017 
Global change drivers, such a climate warming and species invasions, can directly and interactively alter the structure of communities and the functioning of ecosystems. Further, when drivers interact they can exacerbate or buffer the direct effects they each have on ecosystems (Tylianakis et al. 2008, Schweiger et al. 2010, Yang and Rudolf 2010, González-Varo et al. 2013, Sparks and Menzel 2013, Fox et al. 2014). Having a better understanding of how the direct and interactive effects of drivers of global change alter the distribution of species, interactions among species, and the functioning of ecosystems will enable scientists to build and test better predictive models. I experimentally and observationally explored how plant invasion and climate warming interact to alter both the structure of communities and function of ecosystems. I followed up these studies by modeling how species distributions will shift in Tongariro National Park (TNP) and New Zealand, and the implications of such shifts for native species.

\section{Objectives}

I had four overarching objectives that formed the four data chapters of my thesis:

1) To determine whether species-specific phenological shifts have the potential to alter the reproductive capacity of native plants in landscapes invaded by Calluna vulgaris. This formed Chapter 2. I hypothesised that:

a. flowering of the invasive species would be more phenologically plastic than native species of the same growth form, and that this difference in plasticity would lead to altered overlaps in the flowering periods of the invasive and some native species, and

b. lower seed masses of the native would occur in landscapes dominated by invasive flowers, particularly in rich floral landscapes.

2) To determine if the effect of $C$. vulgaris invasion intensity on patterns of native alpine plant species richness is influenced by environmental stress and whether these patterns are consistent across spatial scales. This formed Chapter 3. I hypothesised:

a. that the magnitude of both positive and negative effects of invasion on plant richness at the $1 \mathrm{~m}^{2}$ scale would increase with invader biomass, with the exception of positive interactions at very high invader biomass, and 
b. that, in low stress environments, $z$ - the slope of the Species Area Relationship would increase monotonically with invader canopy cover, whereas in high stress environments the impact of invader canopy cover on $z$ should be unimodal: moderate canopy cover should be associated with higher $z$, and contiguous canopy cover should be associated with a lower $z$ compared to uninvaded communities.

3) To develop a novel modelling framework that would account for density-dependant competitive interactions of a native with an invader and implement this to determine the combined risk of climate change and $C$. vulgaris invasion on the distribution of the native plant species. This formed Chapter 4 . I hypothesised that:

a. under projected future climates in TNP, the geographic distribution of Chionochloa rubra would decrease, while the distribution of $C$. vulgaris would expand, and

b. high C. vulgaris densities would be associated with low $C$. rubra densities, and

c. environmental suitability would be predictive of the upper limit of $C$. vulgaris density, and

d. a large proportion of the distribution of $C$. rubra would overlap with areas in which I would predict high densities of $C$. vulgaris (its impact niche), under both current and future climates.

4) To explore the possible mechanisms leading to a discrepancy in $C$. vulgaris invasion success on the North and South Islands of New Zealand. This formed Chapter 5. I hypothesised that:

a. environmental suitability (climatic, soil pH or a lack of suitable vegetative communities or mycorrhizal mutualists) would not limit $C$. vulgaris invasion at large spatial scales on the South Island, and

b. C. vulgaris occurrences would be significantly associated with a comparable intensity of human disturbance on the two islands despite a significant difference in human impact conditions between the two islands, and further, 
that South Island C. vulgaris occurrences would be spatially clustered around areas of high human disturbance.

\section{Key findings}

Overall, my thesis finds that two global change drivers - warming and non-native plant invasion - work directly and interactively to impact native alpine communities in New Zealand. My data and modeling work suggest that $C$. vulgaris is likely to expand its range, not only in TNP, but across New Zealand in the future. C. vulgaris is particularly likely to adversely affect community structure and ecosystem function in warmer climates. Globally, alpine ecosystems are considered relatively unaffected by invasion (McDougall et al. 2011). However, I present evidence that climate change will facilitate higher rates of species invasion into alpine ecosystems, and that this process can be expedited by increasing human activity. This facilitation may lead to increased invader biomass and canopy cover where species currently occur and may have broad implications for the structure and function of alpine plant communities.

\section{Chapter 2}

In Chapter 2, I predicted that the interaction between climate warming and $C$. vulgaris invasion will reduce sexual reproduction of the native plant, Dracophyllum subulatum, in some landscapes in TNP through a previously undescribed mechanism - intensified competition for pollination because of increased flowering overlap resulting from species-specific phenological responses to climate warming. I observed more phenological plasticity in the invasive plant than the native plant species. The change in the invasive plants' phenology led to a four-fold increase (from $2.79 \%$ to $11.27 \%$ ) in the number of $D$. subulatum flowers that flowered simultaneously with high densities of $C$. vulgaris flowers. Secondly, I found that neither floral density nor the proportion of native flowers in the landscape had significant direct effects on $D$. subulatum seed weight; importantly, I found a significant interaction between these. Lower $D$. subulatum seed weights were recorded in florally-saturated landscapes dominated by invasive flowers, while higher $D$. subulatum seed weights were found in landscapes dominated by native flowers. 
My results reveal a previously undescribed pathway through which climate warming and plant invasion may interact synergistically, enhancing the negative outcomes for sexual reproduction in native species. Invasive species are typically more phenotypically plastic than native species and also produce many showy flowers that can lead to increased competition for pollination (Chittka and Schurkens 2001, Brown et al. 2002, Gallinat et al. 2018). Consequently, the pathway for synergistic effects of invasion and climate warming that I demonstrated may also apply to other species and ecosystems. However, the outcome of this synergism on the sexual reproduction of native species will depend on the direction of change in flowering overlap between native and invasive species. While the effects of climate change on plant competition for pollination has received some theoretical attention (e.g. Schweiger et al. 2010), my results are the first to couple established effects in a novel experimental design to probe at the potential consequences of such an interaction on the reproductive success of an endemic plant species.

\section{Chapter 3}

The response of native communities at small spatial scales was most sensitive to aboveground biomass of the invader, whereas canopy cover was most important for predicting $z$. The sign of native:invasive species interactions at a small scale changed with environmental stress. The magnitude of invader impact on small scale species richness was monotonically determined by biomass of the invader in the case of both competitive and facilitative interactions, with the exception of very high invader biomass in high stress environments. The relationship between invader canopy cover and $z$ was unimodal in both high and low stress environments and changed sign with elevation. Our study suggests that the impact of invasive species on SAR is highly sensitive to environmental stress.

I show that there is a switch in the sign of species interactions from competitive to facilitative with increasing abiotic stress, a trend that is reported globally (Callaway et al. 2002, He et al. 2013). Further, I show that the positive or negative impact of these species interactions is dependent on invader biomass and canopy cover. Uniquely, I show how stressgradient related changes in species interactions modulate the impact of invasive species on 
SAR. The effects of competitive and facilitative interactions affect SAR differently, with competition generally affecting species richness more locally (Powell et al. 2013, Case et al. 2016, Socolar et al. 2016) whereas facilitation may also be an important driver at larger spatial scales (Callaway et al. 2002). Therefore, my novel finding that invasive species effects on $z$ change sign with environmental stress are in line with expectations but have not previously been demonstrated. This finding has global implications for our understanding of how invasive species affect native species diversity patterns across space because it demonstrates that the sign of their effects on species richness may be contingent on climatic context. I record significant shifts in invader biomass with environmental stress, which provides a possible mechanism through which these changes occur; as species traits typically affect species interactions (He et al. 2013, Levine 2016).

The results detailed in Chapter 3 builds on those presented in Chapter 2 by indicating an additional mechanism through which $C$. vulgaris may impact native plant species at larger spatial scales. Sharing animal pollinators is a mechanism through which sessile organisms may interact indirectly with one another at a distance (Mitchell et al. 2009). Such indirect interactions may have important effects for the persistence of native species by reducing their sexual reproduction (Brown et al. 2002), particularly as pollination services to plants in alpine ecosystems such as TNP are typically unreliable (Arroyo et al. 2013).

\section{Chapter 4}

I demonstrate that the inclusion of invasive species density data in distribution models for a native plant leads to greater reductions in predicted native plant distribution under future climate change scenarios relative to models based on climate suitability alone (Chapter 4). My climatic envelope models predicted a range expansion for $C$. vulgaris by 2070 under all future climate change scenarios. Simultaneously, I predicted a range contraction for $C$. rubra under future climates. I found that increasing $C$. vulgaris density was associated with strong declines in $C$. rubra - I recorded an eight-fold decrease in average $C$. rubra density above a critical $C$. vulgaris density threshold. Under all climate scenarios, I forecast increases in the geographic range wherein suitable climatic conditions for high densities of $C$. vulgaris will occur. 
Specifically, I found that up to $93 \%$ of the area where the climate is both suitable for $C$. rubra and where $C$. rubra is currently unaffected by intense competition with $C$. vulgaris will be lost by 2070.

Species extinctions attributed to global warming are often driven by synergisms with invasion, and commonly occur through exacerbated competitive pressure (Brook et al. 2008). Climate change is anticipated to affect plant distributions both directly and indirectly by altering species interactions, although the latter is generally assumed to occur on smaller scales (Wisz et al. 2013). Implementations of existing methodologies, such as Joint Species Distribution Modelling (JSDM; Pollock et al. 2014) often find that species interactions poorly explain the distribution of species (Copenhaver-Parry and Bell 2018). My novel statistical approach may be better suited for the evaluation of risk to native species for two reasons: First, invasive species typically have a disproportionate impact where they occur at high densities (Kulhanek et al. 2011, Bradley 2013) and second, the primary effect of invasive species' competition may be to reduce the density, rather than occurrence, of native species (Copenhaver-Parry and Bell 2018). Both characteristics of my approach result in higher sensitivity of predictions, resulting in more accurate representations of the risk of climate warming and invasive species for native species. Relative to other modelling approaches, my approach is likely to forecast a smaller geographic extent, but higher intensity of invasive plant impact on native species.

My findings here are informed by those presented in Chapter 3, which explicitly addresses issues of scale. The interaction term I used in Chapter 4 to infer competition is derived from density-dependence of $C$. rubra and $C$. vulgaris at a $1 \mathrm{~m}^{2}$ scale and therefore does not investigate the effect of $C$. vulgaris on the range of $C$. rubra, focusing instead on quantifying the $C$. rubra density loss within areas of distributional overlap. In Chapter 3 I detail how interactions between $C$. vulgaris and the native plant community may propagate to larger spatial scales. The results presented in Chapters 3 and 4, taken together, identify that plant invasions may drive declines in native species canopy cover locally, as well as altering their occurrence at multiple spatial scales, which together may exacerbate risk for native species. Rarity in a landscape has important implications for population persistence (Sexton et al. 2009) 
particularly because it may lead to allee effects (Courchamp et al. 1999), while lower canopy cover suggests smaller plants, which can be related to lower fitness. Together, such effects mean increased risk of extinction for non-random species.

The findings of this chapter, in turn, inform Chapter 3. My climate envelope model identified the climatic predictors that constrain both the range and canopy cover of $C$. vulgaris in TNP. I predict that canopy cover of $C$. vulgaris will increase at higher elevation under an altered climate. In Chapter 3, I found that native alpine plant species richness is facilitated by moderate $C$. vulgaris canopy cover. Together, these results suggest a loss of species richness may occur at high elevations. Further, these two chapters indicate that invasion intensity is an important predictor of plant community response irrespective of response variable and support previous assertions that modelling invasive species density explicitly is an imperative development in future modelling studies (VanDerWal et al. 2009, Bradley 2013). This highlights the relevance of the novel modelling methodology I developed in Chapter 4. It also indicates the possibility of extending my approach to investigate other ecological patterns.

Chapters 3 and 4, taken together, also raise further questions. Species interactions will change in response to climate; yet, changes in species interactions are generally poorly incorporated into SDM (Wisz et al. 2013). In Chapter 3, I demonstrate that C. vulgaris has inconsistent effects on native alpine plant community patterns depending on environmental context. This result may be due to variance in $C$. vulgaris aboveground biomass along environmental stress gradients. This implies that the effect of invasion on native species density (as in Chapter 4) may be more accurately modelled by incorporating environmental variability across the calibration range. The density-dependence relationship used in Chapter 4 was calibrated using data which spanned a wide range of environmental conditions. Incorporating these conditions explicitly is likely to reduce unexplained variance within the model. This should result in predictions with a smaller extent, but a higher intensity, of invasive species impacts. However, Chapter 3 does not delve into how environmental stress modulates the effect of $C$. vulgaris on individual species. Therefore, this relationship between the two chapters is speculative. However, the results of these two chapters together highlight the need for future modelling work that incorporates changes in species interactions with environmental 
conditions. Further, my results in Chapter 3 suggest that changing species interactions could be driven by models of plant traits.

\section{Chapter 5}

I find no evidence for large-scale climatic, edaphic, and vegetative limitations to invasion by $C$. vulgaris on either the North and South Islands of New Zealand. C. vulgaris is significantly associated with medium to high levels of human disturbance on both islands despite differences in the presence of human disturbance between the two islands, suggesting that discrepancies in invasive spread between islands may be driven by human activity. I found high climatic suitability for $C$. vulgaris across large areas of both islands. The majority of New Zealand had soil pH within an appropriate range for $C$. vulgaris and large areas of both islands hosted vegetative communities at risk of invasion by $C$. vulgaris. Likewise, native plant species that host ericoid mycorrhizal fungi that form symbioses with $C$. vulgaris were distributed throughout New Zealand. We therefore conclude that $C$. vulgaris is not excluded at large scales by environmental filters. In contrast, mean human impact was significantly higher on the North Island than on the South Island. Despite this discrepancy, I found no significant difference between islands in the mean human impact at $C$. vulgaris occurrences. This suggests that the species occurs disproportionately in similar human disturbance conditions across the two islands. Further, occurrences of $C$. vulgaris on the South Island were non-randomly distributed and were clustered around areas of high human impact. My results suggest that the environmental conditions suitable for $C$. vulgaris invasion are widespread on both islands and further, that human disturbance is likely a major vector by which $C$. vulgaris expands its distribution in New Zealand.

Where environmental conditions are not limiting at large spatial scales, as I record for $C$. vulgaris throughout both islands of New Zealand, invasion biologists predict that currently naturalised species may become problematic invasive species (Jimenez-Valverde et al. 2011), particularly as human population growth and land-use intensification continues. Identifying mechanisms facilitating the expansion of introduced species such as $C$. vulgaris is therefore of critical importance. My findings are useful for conservation managers in New Zealand, as 
invasive species are typically significantly more expensive and difficult to remove once established. My finding, that diverging trajectories of $C$. vulgaris invasion on these two islands of New Zealand are associated with human impact, is in line with recent work that highlights the importance of human activities in dispersing invasive species and facilitating their establishment (Gallardo et al. 2015, Horvitz et al. 2017). Human activities such as road development are rarely incorporated into invasive species distribution models. My works adds to this discourse, by providing evidence that the development of invasive species' distributions may hinge on human activities within a paired island experiment. Internationally, researchers should carefully consider and incorporate a range of human activities in their modelling to achieve better and more accurate modelling outcomes.

Alpine ecosystems globally are threatened by species invasions that are facilitated by climate warming (Alexander et al. 2016, Dainese et al. 2017). Notably, New Zealand is likely to face increased plant invasions under future climates from a pool of introduced species that have already established in the country (Sheppard et al. 2016). Thus, the potential future distribution of $C$. vulgaris detailed in Chapter 5 is supported by my findings in Chapter 4 (i.e., that significant potential mobility of both $C$. vulgaris occurrence and impact niches into high alpine areas in New Zealand may be expected under future climate scenarios). The South Island has a more extensive alpine zone than the North Island (Halloy and Mark 2003), therefore invasion by $C$. vulgaris may have a larger spatial extent of impact on South Island native plant communities under climate change. Alpine plant communities are of significant conservation importance in New Zealand (Clarke et al. 2018) and species are likely to be put at extinction risk by the direct impact of climate change as well as through the expansion of invasive species (Halloy and Mark 2003) such as C. vulgaris. The findings of Chapters 2-4 demonstrate the potential of high-density $C$. vulgaris to constitute a significant ecological risk, by affecting both the structure and function of alpine ecosystems. Taken together these results imply that, in New Zealand, preventative management of $C$. vulgaris is appropriate, particularly where the species has not yet naturalized. 


\section{Summary statement}

My research shows that to make clear predictions of how global change will impact communities and ecosystems in the future, experiments and models need to include interactions among drivers such as warming and species invasion. While many studies highlight the impact of global change drivers on community structure and ecosystem function as well as the fate of individual species in isolation, a growing number of studies, including the ones I present in this thesis, highlight that interactions among drivers may not be additive or linear. Given most landscapes are concurrently affected by multiple drivers of global change, their interactions should be investigated explicitly to produce real predictions of future ecosystem structure and function. 


\section{Strengths and limitations}

One of the strengths of my research is the use of multiple approaches - observations, experiments and models - to explore how global change will interactively alter alpine ecosystems in New Zealand. For example, in Chapter 2, I combined a local-scale manipulative warming experiment on plant phenology with a landscape-scale experiment on pollination limitation to infer the impacts of species-specific shifts in flowering phenology in response to warming on the sexual reproduction of a native species in invaded landscapes. In Chapter 3, I used established survey methodology to investigate invasive plant impacts on SAR, but I placed the plots along elevational gradients, taking advantage of their utility as models of changing abiotic conditions to gain insight to how the impacts of invasion may vary over gradients of environmental stress. In Chapter 4, I used a hierarchical SDM, but derived more informative predictions by pairing large occurrence datasets from public repositories with my own fieldcollected density data within a novel modelling framework. Finally, in Chapter 5, I used established modelling practices for predicting invasion risk but paired this with inter-island comparisons to infer the drivers of $C$. vulgaris spread by eliminating other mechanisms. My research, therefore, exhibits the power of novel combinations of existing approaches to generate new knowledge. While each individual approach may have limitations, using them in combination enables me to play to their strengths in testing and projecting both patterns and mechanisms - thus mitigating the limitations of any one approach.

\section{Chapter 2}

One major limitation of Chapter 2 is that I infer negative consequences of climate warming and plant invasion for the sexual reproduction of a native plant - mediated by interactions with pollinating animals - without examining the response of the insect community to these same drivers of global change. Responses of the insect community to these drivers may modulate the strength of the effect I record here. For example, the phenology of animal pollinators is strongly affected by climate and may affect pollination services (Hegland et al. 2009). Alternatively, animal pollinator populations could be bolstered by novel food sources, as may occur with the introduction of exotic plant species, resulting in overall benefits for the sexual reproduction of native plants (Schweiger et al. 2012, Miller et al. 2018). 
Additional limitations are introduced through the use of Open Top Chambers (OTC) to simulate future climate conditions. OTCs are an established methodology in climate change research (Wolkovich et al. 2012, Arroyo et al. 2013). However, OTCs (like all experimental manipulations) are associated with a number of experimental artefacts (Flanagan et al. 2013) such as mechanical disturbance where they are moved by strong winds. Further, OTCs do not encapsulate the full effects of climatic change, as they do not simulate predicted water balance conditions that can drive changes in alpine communities (Engler et al. 2011). Notably, flowering phenology responds to a suite of environmental cues including both temperature and soil moisture (Godoy et al. 2009, Burkle et al. 2013). Finally, because they are relatively small in size the effect of warming experiments utilizing OTCs may underestimate ecosystem responses to climatic warming (Wolkovich et al. 2012). For these reasons, my results are likely to reflect real responses to future climatic changes but may underestimate flowering phenology responses.

Chapter 2 is further limited by my assumption that seed weight was related to the overall fitness of $D$. subulatum populations. Seed weight in this experiment was unequivocally linked to pollination success, as demonstrated by a significant difference in seed weight between pollinator exclusion and hand pollination treatments. Outcrossing might be critical for the conservation of genetic diversity in populations (Molloy 2019). However, apomixis (the asexual reproduction of seeds) is common in the woody shrub flora of New Zealand and has inconsistent effects on plant population success (Molloy 2019). Therefore, it is unclear to what extent a lack of outcrossing would result in reduced population persistence in $D$. subulatum.

Finally, in Chapter 2 I investigated one mechanistic pathway through which climate change may augment the effects of $C$. vulgaris invasion on the sexual reproduction of $D$. subulatum. However, I did not investigate the direct impacts of climate change or invasion on the sexual reproduction of $D$. subulatum and therefore cannot forecast the risk of global change to the sexual reproduction in this species. For example, climate change may alter the total number of flowers produced by D. subulatum over the course of a season. Additionally, vegetative competition with $C$. vulgaris may affect flower number. I identify these possible mechanisms because both climate change and invasive species alter resource availability that in turn is known to affect allocation to reproduction and the phenology of alpine species (Zhang et 
al. 2014). In this chapter I consider two treatments, ambient and manipulatively warmed climate. However, this study was conducted in a pre-existing, fully factorial experiment with $C$. vulgaris removal as an additional manipulation. Preliminary results indicate that the total flower number over the course of a season decreases with both experimental warming and with the removal of $C$. vulgaris, with the largest decreases recorded with both manipulations (that is, both experimental warming and C. vulgaris removal). A more thorough investigation of these facets of the dataset may provide further insights into the relative roles of climate change, plant-plant interactions and their synergistic effects on the sexual reproduction of native species.

\section{Chapter 3}

A substantial limitation of Chapter 3 is that I did not account for the relative roles of environmental gradients and null processes on the elevational patterns in species richness reported. I report a unimodal relationship of species richness with elevation, a common pattern in many alpine ecosystems (Sanders and Rahbek 2012, Wang et al. 2018), but I did not investigate the mechanisms behind the relationship. This relationship could be an ecological response to environmental factors (Wang et al. 2018). Alternatively, it could reflect null processes such as the geometry of species' ranges in bounded systems (Mid Domain Effect; (Colwell et al. 2004), available area (Vetaas and Grytnes 2002, Xu et al. 2017, Bhatta et al. 2018, Hillebrand et al. 2018) or Rapoport's rule (Zhang et al. 2018) which are all known to structure local richness and the SAR in some ecosystems. Studies in several ecosystems found contradicting patterns in the relative importance of abiotic conditions and null processes (Zhang et al. 2018). Therefore, I cannot make explicit predictions regarding the direct effect of climate change on species richness patterns within the park, nor compare the relative effects of climate and plant invasion on spatial richness patterns. The relative contribution of individual species responses to abiotic conditions and null processes in TNP could be derived without further data collection. My dataset considers not only elevational trends in SAR, which in itself is an understudied pattern (Qiao et al. 2012, Bhatta et al. 2018), but also plant-plant interactions with an invasive species and therefore has considerable potential to add to discourse if null effects were incorporated. 
A general limitation of Chapters 3 and 4 is the use of correlative rather than experimental data to infer process. I describe observations of vegetational patterns along elevational and $C$. vulgaris density gradients but cannot prove that these gradients are the cause of plant richness patterns. It is well established that high densities of one plant species may result in the competitive exclusion of other species. However, co-occurrence patterns may also emerge from niche differences or environmental heterogeneity (Bar-Massada 2015). Biomass compensation experiments unambiguously resolve competitive effects (McLaren and Turkington 2011). In the austral summer of 2016/2017 I established 14 Whittaker plots throughout TNP, and systematically removed aboveground $C$. vulgaris biomass across five of ten $1 \mathrm{~m}^{2}$ plots and one of four $5 \mathrm{~m}^{2}$ plots (a total of $9 \mathrm{~m}^{2}$ at each location), and recorded canopy cover of all vascular plant species. However, when removal and control plots were re-surveyed in 2018 , responses to the removal treatment were not statistically significant. This is likely because growth rates in TNP, as in many alpine ecosystems, are slow (Körner 2013) and species removal experiments often record a significant lag in biomass compensation response (McLaren and Turkington 2011). Therefore, I anticipate that the maintenance of these removal treatments until the ecosystem has sufficient time to respond is a worthy avenue of research.

\section{Chapter 4}

Several limitations arise in Chapter 4 that are common to all SDM. For example, correlative species distribution models assume the species are in dynamic equilibrium with their climatic environment (Nogués-Bravo 2009, Dormann et al. 2012), which is unlikely where the modelling subject is an invasive species, as here. The assumption of correlative models that species can perfectly track the changing geography of their climate space is particularly problematic if species have poor dispersal rates (Araújo and Townsend Peterson 2012, Alexander et al. 2018). I forecast risk to native species by modelling the changing distributional overlap between invasive and native species with the explicit assumption of dynamic equilibrium. Notably, $C$. vulgaris can expand its range quickly in response to climate change (Dainese et al. 2017), whereas the dispersal speed of many native alpine plant species in New Zealand is unknown (Clarke et al. 2018) and is likely to be slow for some alpine species (McGlone et al. 2001). Lag effects may mean that risk to native species is underrepresented in 
my models during transitive phases if natives cannot track climates well at the leading edge of their range. Consequently, although I predict dramatic increases in risk to native plant species from the synergistic impacts of climate change and C. vulgaris invasion, I nevertheless consider my estimates to be conservative. Many reviews consider the limitations of the SDM framework (for examples see Guisan and Thuiller 2005, Thuiller et al. 2008). In addition, predictions of species' distributions under future climate change rely on projected climate data, calculated from Global Circulation Models (GCM), which are associated with considerable uncertainty (Beaumont et al. 2008). Projections are particularly poor for alpine regions in New Zealand (Christian Wild, pers. comm., 2018). In this chapter, I minimized error by selecting GCMs that validate well on current climatic data in the region and by averaging predictions from GCMs, as proposed by Beaumont et al. (2008). This approach has some disadvantages. Averages do not minimise errors that are generally pervasive across GCMs (Beaumont et al. 2008). Additionally, averaging predictions results in the loss of climatic extremes that may be disproportionately important for the delineation of species' ranges (Beaumont et al. 2008, Seddon et al. 2016). However, my predictions nevertheless provide valuable information regarding the relative magnitude of species' responses to climatic changes.

Interactions among species in outlined in Chapter 4 were determined by analyzing densities of co-occurring species using Boosted Regression Trees (BRT). While BRT identified thresholds within the data that were supported by Wilcoxon-signed rank tests, BRT explained very little variance within the dataset. This lack of explained deviance was likely caused by inappropriate model selection. The relationship between invasive plant species density and that of native species may be described as a triangular constraint envelope, where native species density is universally constrained at high invader densities but highly variable at low invader densities. Namely, the relationship is zero-inflated (Lambert 1992). With such a zero-inflated dataset, the inability of BRT - which is based on least sum of squares deviations (Elith et al. 2008) - to describe the bulk of the variation is expected (Martin et al. 2005) as such techniques assume that both presence-absence and abundance components of the relationship come from the same distribution (Zuur et al. 2007). A more appropriate test may be the use of zeroinflated (ZI) models (Martin et al. 2005). ZI methods allow the modelling of different 
relationships for different portions of the range of the response variable (Lambert 1992). First zero counts are modelled in the response variable with a binomial family relationship. Secondly, all positive data $(>0)$ and some zero data are regressed against the predictor variables to determine the driving predictor of species abundance (Bellvé 2018). I envision exploring the use of zero-inflated models for this density-dependence dataset before this body of work goes to publication in an academic journal.

\section{Chapter 5}

My interpretation of ecological processes in Chapter 5 is limited by the number of species (1) and islands (2). This may be problematic in deducing mechanism because stochastic events are disproportionately important for the spread of exotic species in novel ranges (Pielaat et al. 2006). Further, the impact of human activities in Chapter 5 was inferred from one integrated statistic derived from the average responses of 17 species that are invasive in Europe, where environmental conditions and human activities are substantially different from those in New Zealand in many ways. Invasive species spread is correlated with human activity in many regions globally (for example, Horvitz et al. (2017) in China; Gallardo et al. (2015) in Europe) yet it is unclear to what extent such findings may be generalized to New Zealand. Furthermore, different factors are likely to be important at each stage of the invasion process (Theoharides and Dukes 2007), and species that are at early stages of invasion were not considered in that study. C. vulgaris is established on the North Island but to a smaller extent on the South Island, suggesting that human activities on the two islands may affect invasion differently. Further, the use of an integrated statistic elucidates patterns of invasive spread but precludes a mechanistic identification of the human activities that disproportionately facilitate the establishment and spread of this species. It is likely that several human activities facilitate $C$. vulgaris invasion.

A further limitation of this chapter is that the population genetic structure of $C$. vulgaris populations in New Zealand is unknown. Many studies indicate a link between the introduction of new genotypes and increased invasiveness of pre-existing introduced species (Saltonstall 2002, Simberloff 2009, Lucardi et al. 2014, Bock et al. 2015). Novel genotype recombinations in invaded habitats could aid the invasion process and may have occurred in TNP. Indeed, genetic 
diversity has an impact on the fecundity of $C$. vulgaris in Scotland (Miller and Cummins 2001). Consequently, genetic variability may be an explanation, or partial explanation, for the discrepancy in invasive success of $C$. vulgaris in New Zealand. If true, demographically stable South Island populations of $C$. vulgaris could escape from the lag phase through genetic processes; this scenario is increasingly likely as anthropic dispersal pathways increase propagule pressure. This would imply that management policies that limit propagule movement between populations should be favoured. To this end, in 2016, I collected plant tissue from up to 15 individuals at each of 9 South Island populations as well as from throughout TNP and Egmont National Park on the North Island $(n=122)$. While inbreeding depression is unlikely to be the cause of most introduction failures, this dataset would allow estimates of inbreeding and pollen flow, thereby clarifying the role of genetic processes in the occurrence patterns and invasive success of this species.

\section{Future directions}

The research detailed in this thesis was motivated by a desire to contribute to the management of natural places and thereby the conservation of unique landscapes and biodiversity in a changing world. To achieve these management outcomes, future work should focus on integrating multiple interacting drivers of change. Interactions among drivers of change are not limited to those between plant invasion and climate change. For example, in TNP, fire disturbance history has significant impacts on biodiversity patterns and is known to facilitate $C$. vulgaris invasion (Chapman and Bannister 1990, Rogers and Leathwick 1996, Perry et al. 2014) but was not considered within the scope of this thesis. In a similar fashion, atmospheric nitrogen deposition, increasing carbon dioxide concentrations, fragmentation of landscapes, land management practices and many other drivers may interactively impact ecosystem structure and function. The drivers of global change most pertinent to the conservation of landscapes vary globally and among ecosystems. For example, climate change is likely to affect alpine ecosystems globally but may have larger impacts on invasive species spread in the European Alps, where road development is considerable (Dainese et al. 2017). While substantial theoretical work suggests mechanisms whereby drivers of change could 
interact, fewer studies show ecosystem responses empirically, or attempt to model them. This remains a critical avenue for future research.

Interactions among global change drivers can be explored through factorial experiments, vegetative analyses coupled with multivariate analyses, or integration of multiple processes within modelling studies. Such studies are imperative in assessing, for example, how species interactions change under altered climates - these changes are insufficiently understood and are best resolved by factorial experiments and modelling. Work at the interface of subdisciplines provides opportunities to synthesise the outcomes from multiple lines of evidence and to overcome the limitations of individual approaches. For example, interactive effects among drivers are often omitted from empirical studies because they typically operate on different spatial scales. In Chapter 2, I used a mixture of landscape surveys and experimental patterns to identify novel mechanisms of ecosystem change. This synthesis was indispensable: landscape scale patterns in pollinator behaviour could not have been replicated at the scales at which temperature manipulations can be carried out. The incorporation of surveys with experimental work is particularly important, as ecosystems may respond to global change differently at different scales.

Another leading avenue for the integration of multiple drivers of change as well as multiple spatial scales are SDMs. These models are likely to become an increasingly critical component of global change studies. Importantly, SDMs provide ecologists with the ability to forecast ecosystem responses - a necessary precursor to effective conservation management. The predictive capacity of SDM is expanding, driven by increasing data availability and an the infiltration of machine learning from computer science into ecological applications (Elith et al. 2008). However, in the face of this technical development, it is critical that future work focusses on incorporating more biological mechanisms to increase the accuracy of predictions. For example, in Chapter $4 \mathrm{I}$ integrated the effect of climate on both a native and a competitive invasive species. This resulted in a much more negative forecast for the future distribution of the native species. Future work needs to incorporate the responses of multiple facets of ecosystems to global change to provide real estimates of risk for native ecosystems and species. 
To increase their ecological realism, SDMs should make more use of biologically relevant response variables, such as species density rather than species occurrence. Progress in the incorporation of density in SDM is hampered by a scarcity of appropriate data (Nielsen et al. 2005, Kulhanek et al. 2011). Data limitations may be overcome by finding generalities in species and ecosystem responses. For example, I used the relationship between environmental suitability derived from occurrence driven models to predict the abundance of a species thus maximizing the utility of a much larger occurrence dataset (Chapter 4). However, it remains unclear under what circumstances this relationship is predictive. The inclusion of species traits, such as growth form may be more biologically relevant than simple species-level occurrence data (Dallas et al. 2017). Further, the inclusion of other physical variables, such as soil type, could constrain species occurrence-abundance relationships. To make full use of extensive existing species occurrence datasets, future studies should interrogate the conditions under which occurrences are reliably predictive of more biologically meaningful aspects of invasion.

Finally, future work should focus on identifying non-linearities. We find that the magnitude (as with consequences of invasion for the density of native species in Chapter 4) and sign (as with impacts on species richness responses to invasion under changing abiotic stress in Chapter 3) of global change drivers can be driven by non-linear responses. In this context, it is notable that many ecological studies investigate the role of global change by pairing affected or manipulated and control sites. However, ecosystem responses to drivers may respond differently to variable intensities of global change drivers.

Overall, my thesis contributes to a global understanding of community structure and ecosystem function by explicitly investigating the effects of two global change drivers in tandem on native plant communities. Ecosystems worldwide are affected by multiple drivers of change acting in combination and thus it is important for conservation managers to understand the implications of these interactions to manage ecosystems effectively to appropriately mitigate, build resilience, or adapt to changing conditions globally. 


\section{Literature cited}

Abbott, R. J. 2008. History, evolution and future of arctic and alpine flora: overview. Plant Ecology \& Diversity 1:129-133.

Akasaka, M., T. Osawa, and M. Ikegami. 2015. The role of roads and urban area in occurrence of an ornamental invasive weed: a case of Rudbeckia laciniata L. Urban Ecosystems 18:1021-1030.

Alexander, J. M., L. Chalmandrier, J. Lenoir, T. I. Burgess, F. Essl, S. Haider, C. Kueffer, K. McDougall, A. Milbau, M. A. Nuñez, A. Pauchard, W. Rabitsch, L. J. Rew, N. J. Sanders, and L. Pellissier. 2018. Lags in the response of mountain plant communities to climate change. Global Change Biology 24:563-579.

Alexander, J. M., J. J. Lembrechts, L. A. Cavieres, C. Daehler, S. Haider, C. Kueffer, G. Liu, K. McDougall, A. Milbau, A. Pauchard, L. J. Rew, and T. Seipel. 2016. Plant invasions into mountains and alpine ecosystems: current status and future challenges. Alpine Botany 126:89-103.

Araújo, M. B., and A. Guisan. 2006. Five (or so) challenges for species distribution modelling. Journal of Biogeography 33:1677-1688.

Araújo, M. B., and M. New. 2007. Ensemble forecasting of species distributions. Trends in Ecology \& Evolution 22:42-47.

Araújo, M. B., and A. Townsend Peterson. 2012. Uses and misuses of bioclimatic envelope modeling. Ecology 93:1527-1539.

Arrhenius, O. 1921. Species and area. Journal of Ecology 9:95-99.

Arroyo, M. T. K., L. S. Dudley, G. Jespersen, D. A. Pacheco, and L. A. Cavieres. 2013. Temperature-driven flower longevity in a high-alpine species of Oxalis influences reproductive assurance. New Phytologist 200:1260-1268.

Ashcroft, M. B., J. R. Gollan, D. I. Warton, and D. Ramp. 2012. A novel approach to quantify and locate potential microrefugia using topoclimate, climate stability, and isolation from the matrix. Global Change Biology 18:1866-1879.

Atkinson, I. A. E. 1985. Derivation of vegetation mapping units for an ecological survey of Tongariro National North Island, New Zealand. New Zealand Journal of Botany 23:361378.

Atkinson, K. E. 1989. An Introduction to Numerical Analysis. 2nd ed. edition. John Wiley \& Sons, New York.

Auffret, A. G., J. Berg, and S. A. O. Cousins. 2014. The geography of human-mediated dispersal. Diversity and Distributions 20:1450-1456.

Baird, M. F. 2013. 'The breath of the mountain is my heart': indigenous cultural landscapes and the politics of heritage. International Journal of Heritage Studies 19:327-340.

Bannister, P., and W. M. Norton. 1974. Response of mycorrhizal and nonmycorrhizal rooted cuttings of heather (Calluna vulgaris (L) Hull) to variations in nutrient and water regimes New Phytologist 73:81-89.

Bar-Massada, A. 2015. Complex relationships between species niches and environmental heterogeneity affect species co-occurrence patterns in modelled and real communities. Proceedings of the Royal Society B-Biological Sciences 282:8. 
Barbet-Massin, M., F. Jiguet, C. H. Albert, and W. Thuiller. 2012. Selecting pseudo-absences for species distribution models: how, where and how many? Methods in Ecology and Evolution 3:327-338.

Beaumont, L. J., L. Hughes, and A. J. Pitman. 2008. Why is the choice of future climate scenarios for species distribution modelling important? Ecology Letters 11:1135-1146.

Behrend, A., T. Borchert, M. Spiller, and A. Hohe. 2013. AFLP-based genetic mapping of the "bud-flowering" trait in heather (Calluna vulgaris). Bmc Genetics 14.

Bellard, C., J. M. Jeschke, B. Leroy, and G. M. Mace. 2018. Insights from modeling studies on how climate change affects invasive alien species geography. Ecology and Evolution 8:5688-5700.

Bellvé, A. M. 2018. The distribution of epiphytic Astelia and their role in habitat formation. University of Auckland, Auckland.

Beniston, M. 2003. Climatic change in mountain regions: A review of possible impacts. Climatic Change 59:5-31.

Bhatta, K. P., J. A. Grytnes, and O. R. Vetaas. 2018. Scale sensitivity of the relationsh ip between alpha and gamma diversity along an alpine elevation gradient in central Nepal. Journal of Biogeography 45:804-814.

Bjerknes, A.-L., O. Totland, S. J. Hegland, and A. Nielsen. 2007. Do alien plant invasions really affect pollination success in native plant species? Biological Conservation 138:1-12.

Blaauw, B. R., and R. Isaacs. 2014. Larger patches of diverse floral resources increase insect pollinator density, diversity, and their pollination of native wild flowers. Basic and Applied Ecology 15:701-711.

Bliss, L. C. 1962. Adaptations of arctic and alpine plants to environmental conditions. Arctic 15:117-144.

Bock, D. G., C. Caseys, R. D. Cousens, M. A. Hahn, S. M. Heredia, S. Hubner, K. G. Turner, K. D. Whitney, and L. H. Rieseberg. 2015. What we still don't know about invasion genetics. Molecular Ecology 24:2277-2297.

Bokhorst, S., J. W. Bjerke, H. Tommervik, C. Preece, and G. K. Phoenix. 2012. Ecosystem Response to Climatic Change: The Importance of the Cold Season. Ambio 41:246-255.

Bradley, B. A. 2013. Distribution models of invasive plants over-estimate potential impact. Biological Invasions 15:1417-1429.

Bradley, B. A. 2016. Predicting abundance with presence-only models. Landscape Ecology 31:19-30.

Bradley, B. A., J. M. Allen, M. W. O'Neill, R. D. Wallace, C. T. Bargeron, J. A. Richburg, and K. Stinson. 2018. Invasive species risk assessments need more consistent spatial abundance data. Ecosphere 9:10.

Breiman, L. 2001. Random forests. Machine Learning 45:5-32.

Broennimann, O., and A. Guisan. 2008. Predicting current and future biological invasions: both native and invaded ranges matter. Biology Letters 4:585-589.

Brook, B. W., N. S. Sodhi, and C. J. A. Bradshaw. 2008. Synergies among extinction drivers under global change. Trends in Ecology \& Evolution 23:453-460.

Brooker, R. W. 2006. Plant-plant interactions and environmental change. New Phytologist 171:271-284. 
Brown, B. J., R. J. Mitchell, and S. A. Graham. 2002. Competition for pollination between an invasive species (purple loosestrife) and a native congener. Ecology 83:2328-2336.

Brown, J. H. 1984. On the relationhsip between abundance and distribution of species. American Naturalist 124:255-279.

Brusca, R. C., J. F. Wiens, W. M. Meyer, J. Eble, K. Franklin, J. T. Overpeck, and W. Moore. 2013. Dramatic response to climate change in the Southwest: Robert Whittaker's 1963 Arizona Mountain plant transect revisited. Ecology and Evolution 3:3307-3319.

Buckley, L. B., M. C. Urban, M. J. Angilletta, L. G. Crozier, L. J. Rissler, and M. W. Sears. 2010. Can mechanism inform species' distribution models? Ecology Letters 13:1041-1054.

Bullock, J. M., R. J. Edwards, P. D. Carey, and R. J. Rose. 2000. Geographical separation of two Ulex species at three spatial scales: does competition limit species' ranges? Ecography 23:257-271.

Bunn, R. A., P. W. Ramsey, and Y. Lekberg. 2015. Do native and invasive plants differ in their interactions with arbuscular mycorrhizal fungi? A meta-analysis. Journal of Ecology 103:1547-1556.

Burkle, L. A., J. C. Marlin, and T. M. Knight. 2013. Plant-pollinator interactions over 120 Years: loss of species, co-occurrence, and function. Science 339:1611-1615.

Busby, J. R. 1991. BIOCLIM - a bioclimate analysis and prediction system. CSIRO, Australia.

Butterfield, B. J., L. A. Cavieres, R. M. Callaway, B. J. Cook, Z. Kikvidze, C. J. Lortie, R. Michalet, F. I. Pugnaire, C. Schob, S. Xiao, B. Zaitchek, F. Anthelme, R. G. Bjork, K. Dickinson, R. Gavilan, R. Kanka, J. P. Maalouf, J. Noroozi, R. Parajuli, G. K. Phoenix, A. Reid, W. Ridenour, C. Rixen, S. Wipf, L. Zhao, and R. W. Brooker. 2013. Alpine cushion plants inhibit the loss of phylogenetic diversity in severe environments. Ecology Letters 16:478486.

Byers, D. L. 2017. Studying plant-pollinator interactions in a changing climate: a review of approaches. Applications in Plant Sciences 5:15.

Bystriakova, N., M. Peregrym, R. H. J. Erkens, O. Bezsmertna, and H. Schneider. 2012. Sampling bias in geographic and environmental space and its effect on the predictive power of species distribution models. Systematics and Biodiversity 10:305-315.

Caceres, Y., L. D. Llambi, and F. Rada. 2015. Shrubs as foundation species in a high tropical alpine ecosystem: a multi-scale analysis of plant spatial interactions. Plant Ecology \& Diversity 8:147-161.

Callaway, R. M., R. W. Brooker, P. Choler, Z. Kikvidze, C. J. Lortie, R. Michalet, L. Paolini, F. I. Pugnaire, B. Newingham, E. T. Aschehoug, C. Armas, D. Kikodze, and B. J. Cook. 2002. Positive interactions among alpine plants increase with stress. Nature 417:844-848.

Campbell, D. R., M. Bischoff, J. M. Lord, and A. W. Robertson. 2010. Flower color influences insect visitation in alpine New Zealand. Ecology 91:2638-2649.

Carboni, M., I. Calderon-Sanou, L. Pollock, C. Violle, W. Thuiller, and C. DivGrass. 2018. Functional traits modulate the response of alien plants along abiotic and biotic gradients. Global Ecology and Biogeography 27:1173-1185.

Case, E. J., S. Harrison, and H. V. Cornell. 2016. Do high-impact invaders have the strongest negative effects on abundant and functionally similar resident species? Functional Ecology 30:1447-1453. 
Caughley, G., D. Grice, R. Barker, and B. Brown. 1988. The edge of the range. Journal of Animal Ecology 57:771-785.

Cavieres, L. A., R. W. Brooker, B. J. Butterfield, B. J. Cook, Z. Kikvidze, C. J. Lortie, R. Michalet, F. I. Pugnaire, C. Schob, S. Xiao, F. Anthelme, R. G. Bjork, K. J. M. Dickinson, B. H. Cranston, R. Gavilan, A. Gutierrez-Giron, R. Kanka, J. P. Maalouf, A. F. Mark, J. Noroozi, R. Parajuli, G. K. Phoenix, A. M. Reid, W. M. Ridenour, C. Rixen, S. Wipf, L. Zhao, A. Escudero, B. F. Zaitchik, E. Lingua, E. T. Aschehoug, and R. M. Callaway. 2014. Facilitative plant interactions and climate simultaneously drive alpine plant diversity. Ecology Letters 17:193-202.

Chapin, F. S., and G. R. Shaver. 1996. Physiological and growth responses of arctic plants to a field experiment simulating climatic change. Ecology 77:822-840.

Chapin, F. S., E. S. Zavaleta, V. T. Eviner, R. L. Naylor, P. M. Vitousek, H. L. Reynolds, D. U. Hooper, S. Lavorel, O. E. Sala, S. E. Hobbie, M. C. Mack, and S. Diaz. 2000. Consequences of changing biodiversity. Nature 405:234-242.

Chapman, D. S., L. Makra, R. Albertini, M. Bonini, A. Paldy, V. Rodinkova, B. Sikoparija, E. Weryszko-Chmielewska, and J. M. Bullock. 2016. Modelling the introduction and spread of non-native species: international trade and climate change drive ragweed invasion. Global Change Biology 22:3067-3079.

Chapman, H. M., and P. Bannister. 1990. The spread of heather, Calluna vulgaris (L.) Hull, into indigenous plant-communities of Tongariro National Park. New Zealand Journal of Ecology 14:7-16.

Chapman, H. M., and P. Bannister. 1994. Vegetative production and performance of Calluna vulgaris in New Zealand, with particular reference to Tongariro National Park. New Zealand Journal of Ecology 18:109-121.

Chapman, H. M., and P. Bannister. 1995. Flowering, shoot extension, and reproductive performance of heather, Calluna vulgaris (L.) Hull, in Tongariro National Park, New Zealand. New Zealand Journal of Botany 33:111-119.

Chappell, P. R. 2015. The climate and weather of Manawatu-Wanganui. National Institute of Water and Atmospheric Research.

Charlebois, J. A., and R. D. Sargent. 2017. No consistent pollinator-mediated impacts of alien plants on natives. Ecology Letters 20:1479-1490.

Chase, J. M., and T. M. Knight. 2013. Scale-dependent effect sizes of ecological drivers on biodiversity: why standardised sampling is not enough. Ecology Letters 16:17-26.

Chase, J. M., B. J. McGill, D. J. McGlinn, F. May, S. A. Blowes, X. Xiao, T. M. Knight, O. Purschke, and N. J. Gotelli. 2018. Embracing scale-dependence to achieve a deeper understanding of biodiversity and its change across communities. Ecology Letters 21:1737-1751.

Chittka, L., and S. Schurkens. 2001. Successful invasion of a floral market - An exotic Asian plant has moved in on Europe's river-banks by bribing pollinators. Nature 411:653-653.

Choler, P., R. Michalet, and R. M. Callaway. 2001. Facilitation and competition on gradients in alpine plant communities. Ecology 82:3295-3308.

Clark, J. S., S. R. Carpenter, M. Barber, S. Collins, A. Dobson, J. A. Foley, D. M. Lodge, M. Pascual, R. Pielke, W. Pizer, C. Pringle, W. V. Reid, K. A. Rose, O. Sala, W. H. Schlesinger, D. H. Wall, and D. Wear. 2001. Ecological forecasts: An emerging imperative. Science 293:657660. 
Clarke, A. G., J. M. Lord, X. B. Hua, and R. Ohlemuller. 2018. Does current climate explain plant disjunctions? A test using the New Zealand alpine flora. Journal of Biogeography 45:1490-1499.

Classen, A. T., M. K. Sundqvist, J. A. Henning, G. S. Newman, J. A. M. Moore, M. A. Cregger, L. C. Moorhead, and C. M. Patterson. 2015. Direct and indirect effects of climate change on soil microbial and soil microbial-plant interactions: What lies ahead? Ecosphere 6.

Coleman, D. C. 2013. Soil Biota, Soil Systems, and Processes. Pages 580-589 in S. A. Levin, editor. Encyclopedia of Biodiversity (Second Edition). Academic Press, Waltham.

Colwell, R. K., C. Rahbek, and N. J. Gotelli. 2004. The mid-domain effect and species richness patterns: What have we learned so far? American Naturalist 163:E1-E23.

Connor, E. F., and E. D. McCoy. 1979. Statistics and biology of the Species Area Relationship. American Naturalist 113:791-833.

Copenhaver-Parry, P. E., and D. M. Bell. 2018. Species interactions weakly modify climateinduced tree co-occurrence patterns. Journal of Vegetation Science 29:52-61.

Costanza, R., R. dArge, R. deGroot, S. Farber, M. Grasso, B. Hannon, K. Limburg, S. Naeem, R. V. Oneill, J. Paruelo, R. G. Raskin, P. Sutton, and M. vandenBelt. 1997. The value of the world's ecosystem services and natural capital. Nature 387:253-260.

Courchamp, F., T. Clutton-Brock, and B. Grenfell. 1999. Inverse density dependence and the Allee effect. Trends in Ecology \& Evolution 14:405-410.

Cummings, G., S. Anderson, T. Dennis, C. Toth, and S. Parsons. 2014. Competition for pollination by the lesser short-tailed bat and its influence on the flowering phenology of some New Zealand endemics. Journal of Zoology 293:281-288.

Da Silva, E. M., V. M. King, J. L. Russell-Mercier, and R. D. Sargent. 2013. Evidence for pollen limitation of a native plant in invaded communities. Oecologia 172:469-476.

Dainese, M., S. Aikio, P. E. Hulme, A. Bertolli, F. Prosser, and L. Marini. 2017. Human disturbance and upward expansion of plants in a warming climate. Nature Clim. Change 7:577-580.

Dallas, T., R. R. Decker, and A. Hastings. 2017. Species are not most abundant in the centre of their geographic range or climatic niche. Ecology Letters 20:1526-1533.

Danby, R. K., and D. S. Hik. 2007. Variability, contingency and rapid change in recent subarctic alpine tree line dynamics. Journal of Ecology 95:352-363.

Davies, T. J., E. M. Wolkovich, N. J. B. Kraft, N. Salamin, J. M. Allen, T. R. Ault, J. L. Betancourt, K. Bolmgren, E. E. Cleland, B. I. Cook, T. M. Crimmins, S. J. Mazer, G. J. McCabe, S. Pau, J. Regetz, M. D. Schwartz, and S. E. Travers. 2013. Phylogenetic conservatism in plant phenology. Journal of Ecology 101:1520-1530.

De'ath, G., and K. E. Fabricius. 2000. Classification and regression trees: A powerful yet simple technique for ecological data analysis. Ecology 81:3178-3192.

De Caceres, M., and P. Legendre. 2009. Associations between species and groups of sites: indices and statistical inference. Ecology 90:3566-3574.

Descamps, C., L. Moquet, M. Migon, and A.-L. Jacquemart. 2015. Diversity of the insect visitors on Calluna vulgaris (Ericaceae) in Southern France heathlands. Journal of Insect Science 15. 
Dormann, C. F., S. J. Schymanski, J. Cabral, I. Chuine, C. Graham, F. Hartig, M. Kearney, X. Morin, C. Romermann, B. Schroder, and A. Singer. 2012. Correlation and process in species distribution models: bridging a dichotomy. Journal of Biogeography 39:2119-2131.

Drossart, M., D. Michez, and M. Vanderplanck. 2017. Invasive plants as potential food resource for native pollinators: A case study with two invasive species and a generalist bumble bee. Scientific Reports 7:12.

Dvorsky, M., J. Dolezal, M. Kopecky, Z. Chlumska, K. Janatkova, J. Altman, F. de Bello, and K. Rehakova. 2013. Testing the Stress-Gradient Hypothesis at the Roof of the World: Effects of the Cushion Plant Thylacospermum caespitosum on Species Assemblages. Plos One 8.

Ehrenfeld, J. G. 2010. Ecosystem Consequences of Biological Invasions. Annual Review of Ecology, Evolution, and Systematics, Vol 41 41:59-80.

Ekroos, J., A. Jakobsson, J. Wideen, L. Herbertsson, M. Rundlöf, and H. G. Smith. 2015. Effects of landscape composition and configuration on pollination in a native herb: a field experiment. Oecologia 179:509-518.

Elith, J., C. H. Graham, R. P. Anderson, M. Dudik, S. Ferrier, A. Guisan, R. J. Hijmans, F. Huettmann, J. R. Leathwick, A. Lehmann, J. Li, L. G. Lohmann, B. A. Loiselle, G. Manion, C. Moritz, M. Nakamura, Y. Nakazawa, J. M. Overton, A. T. Peterson, S. J. Phillips, K. Richardson, R. Scachetti-Pereira, R. E. Schapire, J. Soberon, S. Williams, M. S. Wisz, and N. E. Zimmermann. 2006. Novel methods improve prediction of species' distributions from occurrence data. Ecography 29:129-151.

Elith, J., M. Kearney, and S. Phillips. 2010. The art of modelling range-shifting species. Methods in Ecology and Evolution 1:330-342.

Elith, J., J. R. Leathwick, and T. Hastie. 2008. A working guide to boosted regression trees. Journal of Animal Ecology 77:802-813.

Engler, R., C. F. Randin, W. Thuiller, S. Dullinger, N. E. Zimmermann, M. B. Araújo, P. B. Pearman, G. Le Lay, C. Piedallu, C. H. Albert, P. Choler, G. Coldea, X. De Lamo, T. Dirnbock, J. C. Gegout, D. Gomez-Garcia, J. A. Grytnes, E. Heegaard, F. Hoistad, D. Nogués-Bravo, S. Normand, M. Puscas, M. T. Sebastia, A. Stanisci, J. P. Theurillat, M. R. Trivedi, P. Vittoz, and A. Guisan. 2011. 21st century climate change threatens mountain flora unequally across Europe. Global Change Biology 17:2330-2341.

ESRI. 2011. ArcGIS Desktop: Release 10. Environmental Systems Research Institute., Redlands, CA.

Evans, A. 2019. Heather beetle gives natives a fighting chance. Pages 6-7 Weed Biocontrol. Landcare Research.

Farrer, E. C., I. W. Ashton, M. J. Spasojevic, S. Y. Fu, D. J. X. Gonzalez, and K. N. Suding. 2015. Indirect effects of global change accumulate to alter plant diversity but not ecosystem function in alpine tundra. Journal of Ecology 103:351-360.

Fattorini, S., P. A. V. Borges, L. Dapporto, and G. Strona. 2017. What can the parameters of the species-area relationship (SAR) tell us? Insights from Mediterranean islands. Journal of Biogeography 44:1018-1028.

Flanagan, L. B., E. J. Sharp, and M. G. Letts. 2013. Response of plant biomass and soil respiration to experimental warming and precipitation manipulation in a Northern Great Plains grassland. Agricultural and Forest Meteorology 173:40-52. 
Fordham, D. A., B. W. Brook, C. Moritz, and D. Nogués-Bravo. 2014. Better forecasts of range dynamics using genetic data. Trends in Ecology \& Evolution 29:436-443.

Fox, C. W. 2018. Towards a mechanistic understanding of global change ecology. Functional Ecology 32:1648-1651.

Fox, R., T. H. Oliver, C. Harrower, M. S. Parsons, C. D. Thomas, and D. B. Roy. 2014. Long-term changes to the frequency of occurrence of British moths are consistent with opposing and synergistic effects of climate and land-use changes. Journal of Applied Ecology 51:949-957.

Franklin, J. 1995. Predictive vegetation mapping: Geographic modelling of biospatial patterns in relation to environmental gradients. Progress in Physical Geography 19:474-499.

Fühner, C., and M. Runge. 2009. Are Fe and P availabilities involved in determining the occurrence and distribution of Calluna vulgaris (L.) Hull in semi-arid grasslands on calcareous soils? Plant and Soil 316:161-176.

Gadgil, M., F. Berkes, and C. Folke. 1993. Indigenous knowledge for biodiversity conservation. Ambio 22:151-156.

Gaertner, M., A. Den Breeyen, C. Hui, and D. M. Richardson. 2009. Impacts of alien plant invasions on species richness in Mediterranean-type ecosystems: a meta-analysis. Progress in Physical Geography 33:319-338.

Gallardo, B., A. Zieritz, and D. C. Aldridge. 2015. The Importance of the Human Footprint in Shaping the Global Distribution of Terrestrial, Freshwater and Marine Invaders. Plos One 10.

Gallinat, A. S., L. Russo, E. K. Melaas, C. G. Willis, and R. B. Primack. 2018. Herbarium specimens show patterns of fruiting phenology in native and invasive plant species across New England. American Journal of Botany 105:31-41.

Gilman, S. E., M. C. Urban, J. Tewksbury, G. W. Gilchrist, and R. D. Holt. 2010. A framework for community interactions under climate change. Trends in Ecology \& Evolution 25:325331.

Godoy, O., P. Castro-Diez, F. Valladares, and M. Costa-Tenorio. 2009. Different flowering phenology of alien invasive species in Spain: evidence for the use of an empty temporal niche? Plant Biology 11:803-811.

González-Varo, J. P., J. C. Biesmeijer, R. Bommarco, S. G. Potts, O. Schweiger, H. G. Smith, I. Steffan-Dewenter, H. Szentgyorgyi, M. Woyciechowski, and M. Vila. 2013. Combined effects of global change pressures on animal-mediated pollination. Trends in Ecology \& Evolution 28:524-530.

Gottfried, M., H. Pauli, A. Futschik, M. Akhalkatsi, P. Barancok, J. L. Benito Alonso, G. Coldea, J. Dick, B. Erschbamer, M. R. Fernandez Calzado, G. Kazakis, J. Krajci, P. Larsson, M. Mallaun, O. Michelsen, D. Moiseev, P. Moiseev, U. Molau, A. Merzouki, L. Nagy, G. Nakhutsrishvili, B. Pedersen, G. Pelino, M. Puscas, G. Rossi, A. Stanisci, J.-P. Theurillat, M. Tomaselli, L. Villar, P. Vittoz, I. Vogiatzakis, and G. Grabherr. 2012. Continent-wide response of mountain vegetation to climate change. Nature Climate Change 2:111-115.

Grassein, F., S. Lavorel, and I. Till-Bottraud. 2014. The importance of biotic interactions and local adaptation for plant response to environmental changes: field evidence along an elevational gradient. Global Change Biology 20:1452-1460. 
Greenwell, B., B. Boehmke, J. Cunningham, and GBM Developers. 2018. gbm: Generalized Boosted Regression Models. Page R package version 2.1.4., https://CRAN.Rproject.org/package =gbm.

Grime, J. P. 1974. Vegetation classificaton by reference to strategies. Nature 250:26-31.

Grinnell, J. 1917. Field tests of theories concerning distributional control. American Naturalist 51:115-128.

Grytnes, J. A. 2003. Species-richness patterns of vascular plants along seven altitudinal transects in Norway. Ecography 26:291-300.

Guisan, A., and W. Thuiller. 2005. Predicting species distribution: offering more than simple habitat models. Ecology Letters 8:993-1009.

Gurevitch, J., G. A. Fox, G. M. Wardle, Inderjit, and D. Taub. 2011. Emergent insights from the synthesis of conceptual frameworks for biological invasions. Ecology Letters 14:407-418.

Halloy, S. R. P., and A. F. Mark. 2003. Climate-change effects on alpine plant biodiversity: A New Zealand perspective on quantifying the threat. Arctic Antarctic and Alpine Research 35:248-254.

Hastie, T. J., and R. Tibshirani. 1990. Generalized additive models. Chapman and Hall.

He, F. L., and S. P. Hubbell. 2011. Species-area relationships always overestimate extinction rates from habitat loss. Nature 473:368-371.

He, Q., M. D. Bertness, and A. H. Altieri. 2013. Global shifts towards positive species interactions with increasing environmental stress. Ecology Letters 16:695-706.

Hector, A., and M. Loreau. 2007. Large-Scale Biodiversity Experiments. Pages 583-589 in S. A. Levin, editor. Encyclopedia of Biodiversity (Second Edition). Academic Press, Waltham.

Hegland, S. J., A. Nielsen, A. Lazaro, A. L. Bjerknes, and O. Totland. 2009. How does climate warming affect plant-pollinator interactions? Ecology Letters 12:184-195.

Heikkinen, R. K., M. Luoto, M. B. Araújo, R. Virkkala, W. Thuiller, and M. T. Sykes. 2006. Methods and uncertainties in bioclimatic envelope modelling under climate change. Progress in Physical Geography 30:751-777.

Hijmans, R. J., S. E. Cameron, J. L. Parra, P. G. Jones, and A. Jarvis. 2005. Very high resolution interpolated climate surfaces for global land areas. International Journal of Climatology 25:1965-1978.

Hijmans, R. J., S. Phillips, J. Leathwick, and J. Elith. 2017. Species Distribution Modeling.

Hill, R. S., G. J. Jordan, and M. K. Macphail. 2015. Why we should retain Nothofagus sensu lato. Australian Systematic Botany 28:190-193.

Hillebrand, H., B. Blasius, E. T. Borer, J. M. Chase, J. A. Downing, B. K. Eriksson, C. T. Filstrup, W. S. Harpole, D. Hodapp, S. Larsen, A. M. Lewandowska, E. W. Seabloom, D. B. Van de Waal, and A. B. Ryabov. 2018. Biodiversity change is uncoupled from species richness trends: Consequences for conservation and monitoring. Journal of Applied Ecology 55:169-184.

Hoover, S. E. R., J. J. Ladley, A. A. Shchepetkina, M. Tisch, S. P. Gieseg, and J. M. Tylianakis. 2012. Warming, CO2, and nitrogen deposition interactively affect a plant-pollinator mutualism. Ecology Letters 15:227-234.

Horvitz, N., R. Wang, F. H. Wan, and R. Nathan. 2017. Pervasive human-mediated large-scale invasion: analysis of spread patterns and their underlying mechanisms in 17 of China's worst invasive plants. Journal of Ecology 105:85-94. 
Hulme, P. E. 2009. Trade, transport and trouble: managing invasive species pathways in an era of globalization. Journal of Applied Ecology 46:10-18.

Ibáñez, I., J. M. Diez, L. P. Miller, J. D. Olden, C. J. B. Sorte, D. M. Blumenthal, B. A. Bradley, C. M. D'Antonio, J. S. Dukes, R. I. Early, E. D. Grosholz, and J. J. Lawler. 2014. Integrated assessment of biological invasions. Ecological Applications 24:25-37.

Inouye, D. W. 2008. Effects of climate change on phenology, frost damage, and floral abundance of montane wildflowers. Ecology 89:353-362.

Inouye, D. W., B. Barr, K. B. Armitage, and B. D. Inouye. 2000. Climate change is affecting altitudinal migrants and hibernating species. Proceedings of the National Academy of Sciences of the United States of America 97:1630-1633.

IPCC. 2014. Climate Change 2014: Synthesis Report. Contribution of Working Groups I, II and III to the Fifth Assessment Report of the Intergovernmental Panel on Climate Change. IPCC, Geneva, Switzerland.

Isaac, J. L., and S. E. Williams. 2013. Climate Change and Extinctions. Pages 73-78 in S. A. Levin, editor. Encyclopedia of Biodiversity (Second Edition). Academic Press, Waltham.

Iverson, L. R., A. M. Prasad, and A. Liaw. 2004. New machine learning tools for predictive vegetation mapping after climate change: Bagging and Random Forest perform better than Regression Tree Analysis. Landscape Ecology of Trees and Forests:317-320.

Jędrzejewska-Szmek, K., and M. Zych. 2013. Flower-visitor and pollen transport networks in a large city: structure and properties. Arthropod-Plant Interactions 7:503-516.

Ji, S. N., A. T. Classen, Z. H. Zhang, and J. S. He. 2017. Asymmetric winter warming advanced plant phenology to a greater extent than symmetric warming in an alpine meadow. Functional Ecology 31:2147-2156.

Jimenez-Valverde, A., A. T. Peterson, J. Soberon, J. M. Overton, P. Aragon, and J. M. Lobo. 2011. Use of niche models in invasive species risk assessments. Biological Invasions 13:27852797.

Johansson, M. 2001. Fungal associations of Danish Calluna vulgaris roots with special reference to ericoid mycorrhiza. Plant and Soil 231:225-232.

Joly, M., P. Bertrand, R. Y. Gbangou, M. C. White, J. Dube, and C. Lavoie. 2011. Paving the Way for Invasive Species: Road Type and the Spread of Common Ragweed (Ambrosia artemisiifolia). Environmental Management 48:514-522.

Jurasinski, G., and J. Kreyling. 2007. Upward shift of alpine plants increases floristic similarity of mountain summits. Journal of Vegetation Science 18:711-718.

Kassambara, A., and F. Mundt. 2017. Extract and Visualize the Results of Multivariate Data Analyses. CRAN.

Keesing, V. F. 1995. Impacts of invasion on community structure: habitat and invertebrate assemblage responses to Calluna vulgaris (L) Hull invasion, Tongariro National Park, New Zealand. Massey University, Palmerston North, New Zealand.

Kharouba, H. M., J. Ehrlen, A. Gelman, K. Bolmgren, J. M. Allen, S. E. Travers, and E. M. Wolkovich. 2018. Global shifts in the phenological synchrony of species interactions over recent decades. Proceedings of the National Academy of Sciences of the United States of America 115:5211-5216.

Kikvidze, Z., R. W. Brooker, B. J. Butterfield, R. M. Callaway, L. A. Cavieres, B. J. Cook, C. J. Lortie, R. Michalet, F. I. Pugnaire, S. Xiao, F. Anthelme, R. G. Bjork, B. H. Cranston, R. G. Gavilan, 
R. Kanka, E. Lingua, J. P. Maalouf, J. Noroozi, R. Parajuli, G. K. Phoenix, A. Reid, W. M. Ridenour, C. Rixen, and C. Schob. 2015. The effects of foundation species on community assembly: a global study on alpine cushion plant communities. Ecology 96:2064-2069.

Kleyer, M., R. M. Bekker, I. C. Knevel, J. P. Bakker, K. Thompson, M. Sonnenschein, P. Poschlod, J. M. van Groenendael, L. Klimes, J. Klimesova, S. Klotz, G. M. Rusch, M. Hermy, D. Adriaens, G. Boedeltje, B. Bossuyt, A. Dannemann, P. Endels, L. Gotzenberger, J. G. Hodgson, A. K. Jackel, I. Kuhn, D. Kunzmann, W. A. Ozinga, C. Romermann, M. Stadler, J. Schlegelmilch, H. J. Steendam, O. Tackenberg, B. Wilmann, J. H. C. Cornelissen, O. Eriksson, E. Garnier, and B. Peco. 2008. The LEDA Traitbase: a database of life-history traits of the Northwest European flora. Journal of Ecology 96:1266-1274.

Knight, T. M., T. L. Ashman, J. M. Bennett, J. H. Burns, S. Passonneau, and J. A. Steets. 2018. Reflections on, and visions for, the changing field of pollination ecology. Ecology Letters 21:1282-1295.

Knouft, J. H. 2018. Appropriate application of information from biodiversity databases is critical when investigating species distributions and diversity: a comment on Dallas et al. (2017). Ecology Letters 21:1119-1120.

Koenker, R., S. Portnoy, P. T. Ng, A. Zeileis, P. Grosjean, and B. D. Ripley. 2018. Package 'quantreg'. CRAN.

Kopp, C. W., and E. E. Cleland. 2018. Plant community response to Artemisia rothrockii (sagebrush) encroachment and removal along an arid elevational gradient. Journal of Vegetation Science 29:859-866.

Körner, C. 2007. The use of 'altitude' in ecological research. Trends in Ecology \& Evolution 22:569-574.

Körner, C. 2013. Alpine Ecosystems. Pages 148-157 in S. A. Levin, editor. Encyclopedia of Biodiversity (Second Edition). Academic Press, Waltham.

Körner, C., J. Paulsen, and E. M. Spehn. 2011. A definition of mountains and their bioclimatic belts for global comparisons of biodiversity data. Alpine Botany 121:73-78.

Kuhn, M. 2018. Classification and Regression Training.

Kulhanek, S. A., B. Leung, and A. Ricciardi. 2011. Using ecological niche models to predict the abundance and impact of invasive species: application to the common carp. Ecological Applications 21:203-213.

Lal, R. 2004. Soil carbon sequestration impacts on global climate change and food security. Science 304:1623-1627.

Laliberte, E., H. Lambers, D. A. Norton, J. M. Tylianakis, and M. A. Huston. 2013. A long-term experimental test of the dynamic equilibrium model of species diversity. Oecologia 171:439-448.

Lambert, D. 1992. Zero-inflated poisson regression, with an application to defects in manufacturing. Technometrics 34:1-14.

Lavergne, S., N. Mouquet, W. Thuiller, and O. Ronce. 2010. Biodiversity and Climate Change: Integrating Evolutionary and Ecological Responses of Species and Communities. Annual Review of Ecology, Evolution, and Systematics, Vol 41 41:321-350.

Levine, J. M. 2016. A trail map for trait-based studies. Nature 529:163-164.

Lord, J. M., A. F. Mark, T. Humar-Maegli, S. R. P. Halloy, P. Bannister, A. Knight, and K. J. M. Dickinson. 2018. Slow community responses but rapid species responses 14 years after 
alpine turf transplantation among snow cover zones, south-central New Zealand. Perspectives in Plant Ecology Evolution and Systematics 30:51-61.

Loreau, M. 2000. Biodiversity and ecosystem functioning: recent theoretical advances. Oikos 91:3-17.

Low, N. H. N., A. Drouin, C. J. Marks, and M. E. S. Bracken. 2015. Invader traits and community context contribute to the recent invasion success of the macroalga Heterosiphonia japonica on New England rocky reefs. Biological Invasions 17:257-271.

Lucardi, R. D., L. E. Wallace, and G. N. Ervin. 2014. Invasion Success in Cogongrass (Imperata cylindrica): A Population Genetic Approach Exploring Genetic Diversity and Historical Introductions. Invasive Plant Science and Management 7:59-75.

MacDougall, A. S., and R. Turkington. 2005. Are invasive species the drivers or passengers of change in degraded ecosystems? Ecology 86:42-55.

Mack, R. N., D. Simberloff, W. M. Lonsdale, H. Evans, M. Clout, and F. A. Bazzaz. 2000. Biotic invasions: causes, epidemiology, global consequences, and control. Ecological Applications 10:689-710.

MacPherson, E. 2017. Regional Gross Domestic Product: Year ended March 2016. Stats NZ, http://archive.stats.govt.nz/browse for stats/economic indicators/NationalAccounts/R egionalGDP HOTPYeMar16.aspx.

Mahy, G., R. A. Ennos, and A. L. Jacquemart. 1999. Allozyme variation and genetic structure of Calluna vulgaris (heather) populations in Scotland: the effect of postglacial recolonization. Heredity 82:654-660.

Mainali, K. P., D. L. Warren, K. Dhileepan, A. McConnachie, L. Strathie, G. Hassan, D. Karki, B. B. Shrestha, and C. Parmesan. 2015. Projecting future expansion of invasive species: comparing and improving methodologies for species distribution modeling. Global Change Biology 21:4464-4480.

Malanson, G. P., and L. M. Resler. 2016. A size-gradient hypothesis for alpine treeline ecotones. Journal of Mountain Science 13:1154-1161.

Mangiafico, S. 2018. Functions to Support Extension Education Program Evaluation. CRAN.

Manthey, M., and J. D. Fridley. 2009. Beta diversity metrics and the estimation of niche width via species co-occurrence data: reply to Zeleny. Journal of Ecology 97:18-22.

Marion, G. M., G. H. R. Henry, D. W. Freckman, J. Johnstone, G. Jones, M. H. Jones, E. Levesque, U. Molau, P. Molgaard, A. N. Parsons, J. Svoboda, and R. A. Virginia. 1997. Open-top designs for manipulating field temperature in high-latitude ecosystems. Global Change Biology 3:20-32.

Mark, A. F. 2012. Above the Treeline: A nature guide to alpine New Zealand. Craig Potton Publishing, Nelson.

Martin, T. G., B. A. Wintle, J. R. Rhodes, P. M. Kuhnert, S. A. Field, S. J. Low-Choy, A. J. Tyre, and H. P. Possingham. 2005. Zero tolerance ecology: improving ecological inference by modelling the source of zero observations. Ecology Letters 8:1235-1246.

Matchett, J. R., P. B. Stark, S. M. Ostoja, R. A. Knapp, H. C. McKenny, M. L. Brooks, W. T. Langford, L. N. Joppa, and E. L. Berlow. 2015. Detecting the influence of rare stressors on rare species in Yosemite National Park using a novel stratified permutation test. Scientific Reports 5:12.

McCullagh, P., and J. A. Nelder. 1989. Generalized linear models. Chapman and Hall. 
McDougall, K. L., A. A. Khuroo, L. L. Loope, C. G. Parks, A. Pauchard, Z. A. Reshi, I. Rushworth, and C. Kueffer. 2011. Plant Invasions in Mountains: Global Lessons for Better Management. Mountain Research and Development 31:380-387.

McGlone, M. S., R. P. Duncan, and P. B. Heenan. 2001. Endemism, species selection and the origin and distribution of the vascular plant flora of New Zealand. Journal of Biogeography 28:199-216.

McLaren, J. R., and R. Turkington. 2011. Biomass compensation and plant responses to 7 years of plant functional group removals. Journal of Vegetation Science 22:503-515.

McNabb, R. F. R. 1961. Mycorrhiza in the New Zealand Ericales. Australian Journal of Botany 9:57-61.

Meunier, G., and C. Lavoie. 2012. Roads as Corridors for Invasive Plant Species: New Evidence from Smooth Bedstraw (Galium mollugo). Invasive Plant Science and Management 5:92100.

Michalet, R., C. Schob, C. J. Lortie, R. W. Brooker, and R. M. Callaway. 2014. Partitioning net interactions among plants along altitudinal gradients to study community responses to climate change. Functional Ecology 28:75-86.

Michelan, T. S., S. M. Thomaz, F. M. Bando, and L. M. Bini. 2018. Competitive Effects Hinder the Recolonization of Native Species in Environments Densely Occupied by One Invasive Exotic Species. Frontiers in Plant Science $\mathbf{9}$.

Milbau, A., J. C. Stout, B. J. Graae, and I. Nijs. 2009. A hierarchical framework for integrating invasibility experiments incorporating different factors and spatial scales. Biological Invasions 11:941-950.

Miller, C. M., B. I. P. Barratt, K. J. M. Dickinson, and J. M. Lord. 2018. Are introduced plants a threat to native pollinator services in montane-alpine environments? Alpine Botany 128:179-189.

Miller, G. R., and R. P. Cummins. 2001. Geographic variation in seed-setting by heather (Calluna vulgaris (L.) Hull) in the Scottish Highlands. Journal of Biogeography 28:1023-1031.

Ministry for the Environment. 2018. Climate Change Projections for New Zealand: Atmosphere Projections Based on Simulations from the IPCC Fifth Assessment, 2nd Edition. . Ministry for the Environment, Wellington.

Mitchell, R. J., R. J. Flanagan, B. J. Brown, N. M. Waser, and J. D. Karron. 2009. New frontiers in competition for pollination. Annals of Botany 103:1403-1413.

Mohan, J. E., C. C. Cowden, P. Baas, A. Dawadi, P. T. Frankson, K. Helmick, E. Hughes, S. Khan, A. Lang, M. Machmuller, M. Taylor, and C. A. Witt. 2014. Mycorrhizal fungi mediation of terrestrial ecosystem responses to global change: mini-review. Fungal Ecology 10:3-19.

Molloy, B. P. J. 2019. Apomixis in indigenous New Zealand woody seed plants and its ecological and wider significance: a working hypothesis. New Zealand Journal of Ecology 43.

Molloy, L. 2015. Protected areas. Te Ara - the Encyclopedia of New Zealand, http://www.TeAra.govt.nz/en/protected-areas/print.

Morales, C. L., and A. Traveset. 2009. A meta-analysis of impacts of alien vs. native plants on pollinator visitation and reproductive success of co-flowering native plants. Ecology Letters 12:716-728. 
Morisette, J. T., C. S. Jarnevich, A. Ullah, W. J. Cai, J. A. Pedelty, J. E. Gentle, T. J. Stohlgren, and J. L. Schnase. 2006. A tamarisk habitat suitability map for the continental United States. Frontiers in Ecology and the Environment 4:11-17.

Mullan, B., A. Sood, S. Stuart, and T. Carey-Smith. 2018. Climate Change Projections for New Zealand: Atmosphere: Projections Based on Simulations from the IPCC Fifth Assessment. Ministry for the Environment, Wellington.

Murphy, C., and A. Robertson. 2000. Preliminary study of the effects of honey bees (Apis mellifera) in Tongariro National Park. Science for Conservation 139.

Nathan, R. 2008. Long-distance dispersal of plants (11 August, pg 786, 2006). Science 322:375375.

Newsome, P., J. Shepherd, and D. Pairman. 2012. LUCAS NZ Land Use Map 199020082012 (v018).in M. f. t. Environment, editor., https://data.mfe.govt.nz.

Nickless, E. M., C. W. N. Anderson, G. Hamilton, J. M. Stephens, and J. Wargent. 2017. Soil influences on plant growth, floral density and nectar yield in three cultivars of mnuka (Leptospermum scoparium). New Zealand Journal of Botany 55:100-117.

Nielsen, J. A., P. A. Whigham, R. D. Frew, R. M. Callaway, and K. J. M. Dickinson. 2015. Thyme and space invasion: Thymus vulgaris decreases exotic plant species diversity in Central Otago, New Zealand. Biological Invasions 17:2261-2274.

Nielsen, S. E., C. J. Johnson, D. C. Heard, and M. S. Boyce. 2005. Can models of presenceabsence be used to scale abundance? - Two case studies considering extremes in life history. Ecography 28:197-208.

Nogués-Bravo, D. 2009. Predicting the past distribution of species climatic niches. Global Ecology and Biogeography 18:521-531.

Nuñez, M. A., T. R. Horton, and D. Simberloff. 2009. Lack of belowground mutualisms hinders Pinaceae invasions. Ecology 90:2352-2359.

Orlovich, D. A., and J. W. G. Cairney. 2004. Ectomycorrhizal fungi in New Zealand: current perspectives and future directions. New Zealand Journal of Botany 42:721-738.

Pairon, M., B. Petitpierre, M. Campbell, A. Guisan, O. Broennimann, P. V. Baret, A. L. Jacquemart, and G. Besnard. 2010. Multiple introductions boosted genetic diversity in the invasive range of black cherry (Prunus serotina; Rosaceae). Annals of Botany 105:881-890.

Panetta, F. D., and B. Gooden. 2017. Managing for biodiversity: impact and action thresholds for invasive plants in natural ecosystems. Neobiota:53-66.

Parmesan, C. 2006. Ecological and evolutionary responses to recent climate change. Pages 637669 Annual Review of Ecology Evolution and Systematics. Annual Reviews, Palo Alto.

Parmesan, C., and G. Yohe. 2003. A globally coherent fingerprint of climate change impacts across natural systems. Nature 421:37-42.

Peknicova, J., and K. Berchova-Bimova. 2016. Application of species distribution models for protected areas threatened by invasive plants. Journal for Nature Conservation 34:1-7.

Perry, G. L. W., J. M. Wilmshurst, and M. S. McGlone. 2014. Ecology and long-term history of fire in New Zealand. New Zealand Journal of Ecology 38:157-176.

Petitpierre, B., K. McDougall, T. Seipel, O. Broennimann, A. Guisan, and C. Kueffer. 2016. Will climate change increase the risk of plant invasions into mountains? Ecological Applications 26:530-544. 
Pielaat, A., M. A. Lewis, S. Lele, and T. de-Camino-Beck. 2006. Sequential sampling designs for catching the tail of dispersal kernels. Ecological Modelling 190:205-222.

Pinheiro, J., D. Bates, S. DebRoy, D. Sarkar, and R. C. Team. 2018. nlme: Linear and Nonlinear Mixed Effects Models. https://CRAN.R-project.org/package=nlme.

Pohl, M., D. Alig, C. Körner, and C. Rixen. 2009. Higher plant diversity enhances soil stability in disturbed alpine ecosystems. Plant and Soil 324:91-102.

Pollock, L. J., R. Tingley, W. K. Morris, N. Golding, R. B. O'Hara, K. M. Parris, P. A. Vesk, and M. A. McCarthy. 2014. Understanding co-occurrence bymodelling species simultaneously with a Joint Species DistributionModel (JSDM). Methods in Ecology and Evolution 5:397-406.

Popic, T. J., G. M. Wardle, and Y. C. Davila. 2013. Flower-visitor networks only partially predict the function of pollen transport by bees. Austral Ecology 38:76-86.

Potts, S. G., J. C. Biesmeijer, C. Kremen, P. Neumann, O. Schweiger, and W. E. Kunin. 2010. Global pollinator declines: trends, impacts and drivers. Trends in Ecology \& Evolution 25:345-353.

Powell, K. I., J. M. Chase, and T. M. Knight. 2013. Invasive Plants Have Scale-Dependent Effects on Diversity by Altering Species-Area Relationships. Science 339:316-318.

Primack, R. B. 1980. Variation in the phenology of natural populations of montane shrubs in New Zealand. Journal of Ecology 68:849-862.

Primack, R. B. 1983. Insect pollination in the New Zealand mountain flora. New Zealand Journal of Botany 21:317-333.

Qiao, X. J., Z. Y. Tang, Z. H. Shen, and J. Y. Fang. 2012. What causes geographical variation in the species-area relationships? A test from forests in China. Ecography 35:1110-1116.

R Development Core Team. 2016. R: A language and environment for statistical computing. $R$ Foundation for Statistical Computing, Vienna, Austria.

Ransijn, J., C. Damgaard, and I. K. Schmidt. 2015. Do competitive interactions in dry heathlands explain plant abundance patterns and species coexistence? Plant Ecology 216:199-211.

Rapson, G. L., and J. B. Wilson. 1992. Genecology of Agrostis capillaris L (Poaceae) - an invader into New Zealand. 1. Floral phenology. New Zealand Journal of Botany 30:1-11.

Reitsma, L. 1994. The frost resistance of some native plants from the Central Volcanic Plateau, North Island, New Zealand, in relation to plant succession. New Zealand Journal of Botany 32:217-226.

Rejmanek, M., and T. J. Stohlgren. 2015. Scale-dependent impacts of invasive species: a reply to Chase et al. (2015). Biology Letters 11.

Rendell, S., and R. A. Ennos. 2002. Chloroplast DNA diversity in Calluna vulgaris (heather) populations in Europe. Molecular Ecology 11:69-78.

Ripley, B. D. 1996. Pattern recognition and neural networks. Cambridge University Press.

Rixen, C., and C. P. H. Mulder. 2009. Species removal and experimental warming in a subarctic tundra plant community. Oecologia 161:173-186.

Robin, X., N. Turck, A. Hainard, N. Tiberti, F. Lisacek, J.-C. Sanchez, and M. Mueller. 2011. pROC: an open-source package for $\mathrm{R}$ and $\mathrm{S}$ plus to analyze and compare ROC curves. BmC Bioinformatics 12.

Rodriguez, L. F. 2006. Can invasive species facilitate native species? Evidence of how, when, and why these impacts occur. Biological Invasions 8:927-939. 
Rogers, G. M., and J. R. Leathwick. 1996. North Island seral tussock grasslands. 3. The influence of heather (Calluna vulgaris) on rates of change from tussock grassland to shrubland. New Zealand Journal of Botany 34:473-487.

Rojas, I. M., and J. B. Zedler. 2015. An invasive exotic grass reduced sedge meadow species richness by half. Wetlands Ecology and Management 23:649-663.

Root, T. L., S. H. Schneider, R. Warren, J. R. Price, and P. R. Mastrandrea. 2013. Climate Change and Wild Species. Pages 79-99 in S. A. Levin, editor. Encyclopedia of Biodiversity (Second Edition). Academic Press, Waltham.

Rouget, M., and D. M. Richardson. 2003. Inferring process from pattern in plant invasions: A semimechanistic model incorporating propagule pressure and environmental factors. American Naturalist 162:713-724.

Saltonstall, K. 2002. Cryptic invasion by a non-native genotype of the common reed, Phragmites australis, into North America. Proceedings of the National Academy of Sciences of the United States of America 99:2445-2449.

Sanders, N. J., and C. Rahbek. 2012. The patterns and causes of elevational diversity gradients. Ecography 35:1-3.

Satterfield, T., R. Gregory, S. Klain, M. Roberts, and K. M. Chan. 2013. Culture, intangibles and metrics in environmental management. Journal of Environmental Management 117:103-114.

Schreiber, S. J., and J. O. Lloyd-Smith. 2009. Invasion Dynamics in Spatially Heterogeneous Environments. American Naturalist 174:490-505.

Schweiger, O., J. C. Biesmeijer, R. Bommarco, T. Hickler, P. E. Hulme, S. Klotz, I. Kuhn, M. Moora, A. Nielsen, R. Ohlemuller, T. Petanidou, S. G. Potts, P. Pysek, J. C. Stout, M. T. Sykes, T. Tscheulin, M. Vila, G. R. Walther, C. Westphal, M. Winter, M. Zobel, and J. Settele. 2010. Multiple stressors on biotic interactions: how climate change and alien species interact to affect pollination. Biological Reviews 85:777-795.

Schweiger, O., R. K. Heikkinen, A. Harpke, T. Hickler, S. Klotz, O. Kudrna, I. Kuhn, J. Poyry, and J. Settele. 2012. Increasing range mismatching of interacting species under global change is related to their ecological characteristics. Global Ecology and Biogeography 21:88-99.

Seddon, A. W. R., M. Macias-Fauria, P. R. Long, D. Benz, and K. J. Willis. 2016. Sensitivity of global terrestrial ecosystems to climate variability. Nature 531:229-+.

Sexton, J. P., P. J. Mclntyre, A. L. Angert, and K. J. Rice. 2009. Evolution and Ecology of Species Range Limits. Pages 415-436 Annual Review of Ecology Evolution and Systematics. Annual Reviews, Palo Alto.

Sheppard, C. S., B. R. Burns, and M. C. Stanley. 2016. Future-proofing weed management for the effects of climate change: is New Zealand underestimating the risk of increased plant invasions? New Zealand Journal of Ecology 40:398-405.

Sheppard, C. S., M. Carboni, F. Essl, H. Seebens, W. Thuiller, and C. DivGrass. 2018. It takes one to know one: Similarity to resident alien species increases establishment success of new invaders. Diversity and Distributions 24:680-691.

Shields, J. M., M. A. Jenkins, M. R. Saunders, H. Zhang, L. H. Jenkins, and A. M. Parks. 2014. Age Distribution and Spatial Patterning of an Invasive Shrub in Secondary Hardwood Forests. Forest Science 60:830-840. 
Simberloff, D. 2009. The Role of Propagule Pressure in Biological Invasions. Annual Review of Ecology Evolution and Systematics 40:81-102.

Simpson, G. L. 2016. Functions for Generating Restricted Permutations of Data.

Smale, M. C. 1990. Ecology of Dracophyllum subulatum-dominant heathland on frost flats at Rangitaiki and North Pureora, central North Island, New Zealand. New Zealand Journal of Botany 28:225-248.

Socolar, J. B., J. J. Gilroy, W. E. Kunin, and D. P. Edwards. 2016. How Should Beta-Diversity Inform Biodiversity Conservation? Trends in Ecology \& Evolution 31:67-80.

Soons, M. B., and J. M. Bullock. 2008. Non-random seed abscission, long-distance wind dispersal and plant migration rates. Journal of Ecology 96:581-590.

Sparks, T., and A. Menzel. 2013. Plant phenology changes and climate change. Pages 103-108 in S. A. Levin, editor. Encyclopedia of Biodiversity (Second Edition). Academic Press, Waltham.

Spasojevic, M. J., and K. N. Suding. 2012. Inferring community assembly mechanisms from functional diversity patterns: the importance of multiple assembly processes. Journal of Ecology 100:652-661.

Statistics New Zealand. 2017. Subnational population estimates (UA, AU), by age and sex, at 30 June 1996, 2001, 2006-16 (2017 boundary). Statistics New Zealand, nzdotstat.stats.govt.nz/.

Steer, M. A., and D. A. Norton. 2013. Factors influencing abundance of invasive hawkweeds, Hieracium species, in tall tussock grasslands in the Canterbury high country. New Zealand Journal of Botany 51:61-70.

Stohlgren, T. J., and M. Rejmanek. 2014. No universal scale-dependent impacts of invasive species on native plant species richness. Biology Letters 10.

Storch, D., K. L. Evans, and K. J. Gaston. 2005. The species-area-energy relationship. Ecology Letters 8:487-492.

Syrett, P., L. A. Smith, T. C. Bourner, S. V. Fowler, and A. Wilcox. 2000. A European pest to control a New Zealand weed: investigating the safety of heather beetle, Lochmaea suturalis (Coleoptera : Chrysomelidae) for biological control of heather, Calluna vulgaris. Bulletin of Entomological Research 90:169-178.

Taiepa, T., P. Lyver, P. Horsley, J. Davis, M. Bragg, and H. Moller. 1997. Co-management of NewZealand's conservation estate by Maori and Pakeha: a review. Environmental Conservation 24:236-250.

The Team of Tongariro/Taupō Conservancy. 2006. Tongariro/Taupō Conservation Management Planning Series 4. Pages 1- 324 in D. o. Conservation, editor., Wellington, New Zealand.

Theobald, E. J., H. Gabrielyan, and J. HilleRisLambers. 2016. Lilies at the limit: Variation in plantpollinator interactions across an elevational range. American Journal of Botany 103:189197.

Theoharides, K. A., and J. S. Dukes. 2007. Plant invasion across space and time: factors affecting nonindigenous species success during four stages of invasion. New Phytologist 176:256273.

Therneau, T., and B. Atkinson. 2018. rpart: Recursive Partitioning and Regression Trees. Pages $\mathrm{R}$ package version 4.1-13, https://CRAN.R-project.org/package=rpart. 
Thompson, A. H., and T. M. Knight. 2018. Exotic plant species receive adequate pollinator service despite variable integration into plant-pollinator networks. Oecologia 187:135142.

Thuiller, W., B. Lafourcade, R. Engler, and M. B. Araújo. 2009. BIOMOD - a platform for ensemble forecasting of species distributions. Ecography 32:369-373.

Timmins, S. M., and P. A. Williams. 1991. Weed numbers in New Zealand forest and scrub reserves. New Zealand Journal of Ecology 15:153-162.

Traveset, A., G. Brundu, L. Carta, I. Mprezetou, P. Lambdon, M. Manca, F. Medail, E. Moragues, J. Rodriguez-Perez, A. S. D. Siamantziouras, C. M. Suehs, A. Y. Troumbis, M. Vila, and P. E. Hulme. 2008. Consistent performance of invasive plant species within and among islands of the Mediterranean basin. Biological Invasions 10:847-858.

Tylianakis, J. M., R. K. Didham, J. Bascompte, and D. A. Wardle. 2008. Global change and species interactions in terrestrial ecosystems. Ecology Letters 11:1351-1363.

Urcelay, C., S. Longo, J. Geml, P. A. Tecco, and E. Nouhra. 2017. Co-invasive exotic pines and their ectomycorrhizal symbionts show capabilities for wide distance and altitudinal range expansion. Fungal Ecology 25:50-58.

Van Couwenberghe, R., C. Collet, J. C. Pierrat, K. Verheyen, and J. C. Gegout. 2013. Can species distribution models be used to describe plant abundance patterns? Ecography 36:665674.

VanDerWal, J., L. P. Shoo, C. N. Johnson, and S. E. Williams. 2009. Abundance and the Environmental Niche: Environmental Suitability Estimated from Niche Models Predicts the Upper Limit of Local Abundance. American Naturalist 174:282-291.

Veloz, S., L. Salas, B. Altman, J. Alexander, D. Jongsomjit, N. Elliott, and G. Ballard. 2015. Improving effectiveness of systematic conservation planning with density data. Conservation Biology 29:1217-1227.

Vetaas, O. R., and J. A. Grytnes. 2002. Distribution of vascular plant species richness and endemic richness along the Himalayan elevation gradient in Nepal. Global Ecology and Biogeography 11:291-301.

Vila, M., J. L. Espinar, M. Hejda, P. E. Hulme, V. Jarosik, J. L. Maron, J. Pergl, U. Schaffner, Y. Sun, and P. Pysek. 2011. Ecological impacts of invasive alien plants: a meta-analysis of their effects on species, communities and ecosystems. Ecology Letters 14:702-708.

Vitousek, P. M., H. A. Mooney, J. Lubchenco, and J. M. Melillo. 1997. Human domination of Earth's ecosystems. Science 277:494-499.

Vittoz, P., D. Cherix, Y. Gonseth, V. Lubini, R. Maggini, N. Zbinden, and S. Zumbach. 2013. Climate change impacts on biodiversity in Switzerland: A review. Journal for Nature Conservation 21:154-162.

Walker, K. J., E. A. Warman, A. Bhogal, R. B. Cross, R. F. Pywell, B. R. Meek, B. J. Chambers, and R. Pakeman. 2007. Recreation of lowland heathland on ex-arable land: assessing the limiting processes on two sites with contrasting soil fertility and $\mathrm{pH}$. Journal of Applied Ecology 44:573-582.

Walther, G. R., E. Post, P. Convey, A. Menzel, C. Parmesan, T. J. C. Beebee, J. M. Fromentin, O. Hoegh-Guldberg, and F. Bairlein. 2002. Ecological responses to recent climate change. Nature 416:389-395. 
Wang, J. H., Y. F. Cai, L. Zhang, C. K. Xu, and S. B. Zhang. 2018. Species Richness of the Family Ericaceae along an Elevational Gradient in Yunnan, China. Forests 9.

Wardle, D. A., and O. Zackrisson. 2005. Effects of species and functional group loss on island ecosystem properties. Nature 435:806-810.

Wardle, P. 1991. Vegetation of New Zealand. Cambridge University Press.

Watling, J. I., D. N. Bucklin, C. Speroterra, L. A. Brandt, F. J. Mazzotti, and S. S. Romanach. 2013. Validating Predictions from Climate Envelope Models. Plos One 8.

Watt, A. S. 1955. Bracken versus heather, a study in plant sociology. Journal of Ecology 43:490506.

Weber, M. M., R. D. Stevens, J. A. F. Diniz, and C. E. V. Grelle. 2017. Is there a correlation between abundance and environmental suitability derived from ecological niche modelling? A meta-analysis. Ecography 40:817-828.

Weppler, T., P. Stoll, and J. Stocklin. 2006. The relative importance of sexual and clonal reproduction for population growth in the long-lived alpine plant Geum reptans. Journal of Ecology 94:869-879.

Whittaker, R. H. 1967. Gradient analysis of vegetation. Biological Reviews of the Cambridge Philosophical Society 42:207-\&.

Wilber, M. Q., J. Kitzes, and J. Harte. 2015. Scale collapse and the emergence of the power law species-area relationship. Global Ecology and Biogeography 24:883-895.

Wilson, J. B., and C. D. Meurk. 2011. The control of community composition by distance, environment and history: a regional-scale study of the mountain grasslands of southern New Zealand. Journal of Biogeography 38:2384-2396.

Winkworth, R. C., S. J. Wagstaff, D. Glenny, and P. J. Lockhart. 2005. Evolution of the New Zealand mountain flora: Origins, diversification and dispersal. Organisms Diversity \& Evolution 5:237-247.

Winstral, A., K. Elder, and R. E. Davis. 2002. Spatial snow modeling of wind-redistributed snow using terrain-based parameters. Journal of Hydrometeorology 3:524-538.

Wisz, M. S., J. Pottier, W. D. Kissling, L. Pellissier, J. Lenoir, C. F. Damgaard, C. F. Dormann, M. C. Forchhammer, J. A. Grytnes, A. Guisan, R. K. Heikkinen, T. T. Hoye, I. Kuhn, M. Luoto, L. Maiorano, M. C. Nilsson, S. Normand, E. Ockinger, N. M. Schmidt, M. Termansen, A. Timmermann, D. A. Wardle, P. Aastrup, and J. C. Svenning. 2013. The role of biotic interactions in shaping distributions and realised assemblages of species: implications for species distribution modelling. Biological Reviews 88:15-30.

Wolkovich, E. M., and E. E. Cleland. 2014. Phenological niches and the future of invaded ecosystems with climate change. Aob Plants 6:16.

Wolkovich, E. M., B. I. Cook, J. M. Allen, T. M. Crimmins, J. L. Betancourt, S. E. Travers, S. Pau, J. Regetz, T. J. Davies, N. J. B. Kraft, T. R. Ault, K. Bolmgren, S. J. Mazer, G. J. McCabe, B. J. McGill, C. Parmesan, N. Salamin, M. D. Schwartz, and E. E. Cleland. 2012. Warming experiments underpredict plant phenological responses to climate change. Nature 485:494-497.

Wood, S. 2018. Mixed GAM Computation Vehicle with Automatic Smoothness Estimation. CRAN. 
Wood, S. N. 2011. Fast stable restricted maximum likelihood and marginal likelihood estimation of semiparametric generalized linear models. Journal of the Royal Statistical Society Series B-Statistical Methodology 73:3-36.

Wu, Z., P. Dijkstra, G. W. Koch, J. Penuelas, and B. A. Hungate. 2011. Responses of terrestrial ecosystems to temperature and precipitation change: a meta-analysis of experimental manipulation. Global Change Biology 17:927-942.

Xu, X., H. Y. Zhang, J. Luo, D. J. Zhang, and A. Ma. 2017. Area-corrected species richness patterns of vascular plants along a tropical elevational gradient. Journal of Mountain Science 14:694-704.

Yang, L. H., and V. H. W. Rudolf. 2010. Phenology, ontogeny and the effects of climate change on the timing of species interactions. Ecology Letters 13:1-10.

Zhang, G. F., W. Z. Zhao, H. Zhou, Q. Y. Yang, and X. F. Wang. 2018. Extreme drought stress shifts net facilitation to neutral interactions between shrubs and sub-canopy plants in an arid desert. Oikos 127:381-391.

Zhang, Z. L., K. C. Niu, X. D. Liu, P. Jia, and G. Z. Du. 2014. Linking flowering and reproductive allocation in response to nitrogen addition in an alpine meadow. Journal of Plant Ecology 7:231-239.

Zuur, A., E. N. leno, and G. M. Smith. 2007. Analyzing ecological data. Springer Science \& Business Media. 


\section{Appendix 1: Supplementary materials to Chapter 2}

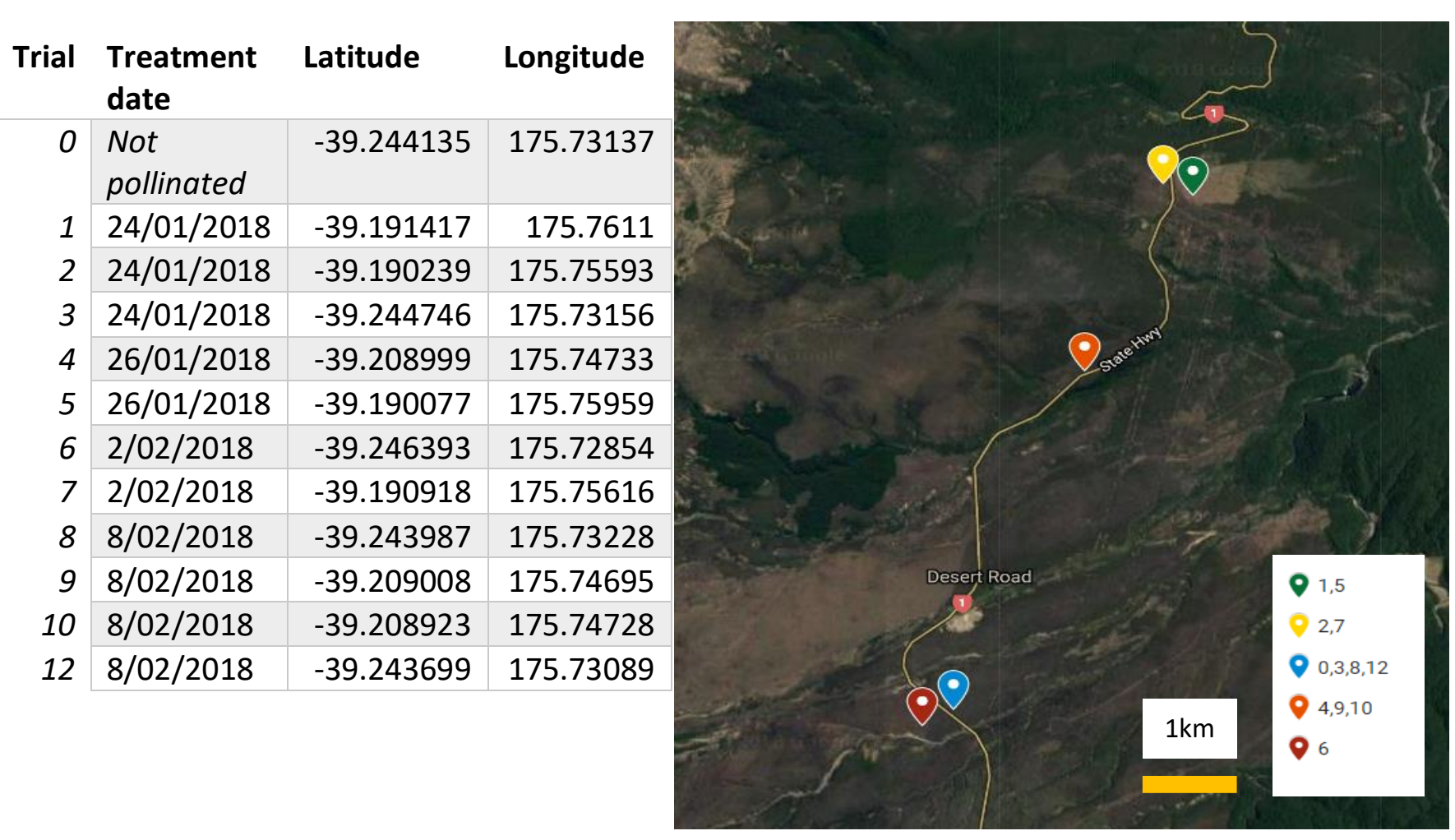

Supplementary Figure 1: Timing and location of pollination trials. Trials were carried within five blocks of land adjacent to Tongariro National Park, New Zealand which are indicated on the map. The number of each trial at each of these five locations is provided in the legend. Within each block, trials were separated by a minimum of $500 \mathrm{~m}$ distance if running concurrently. Accurate locations of each trial are provided in the table. 


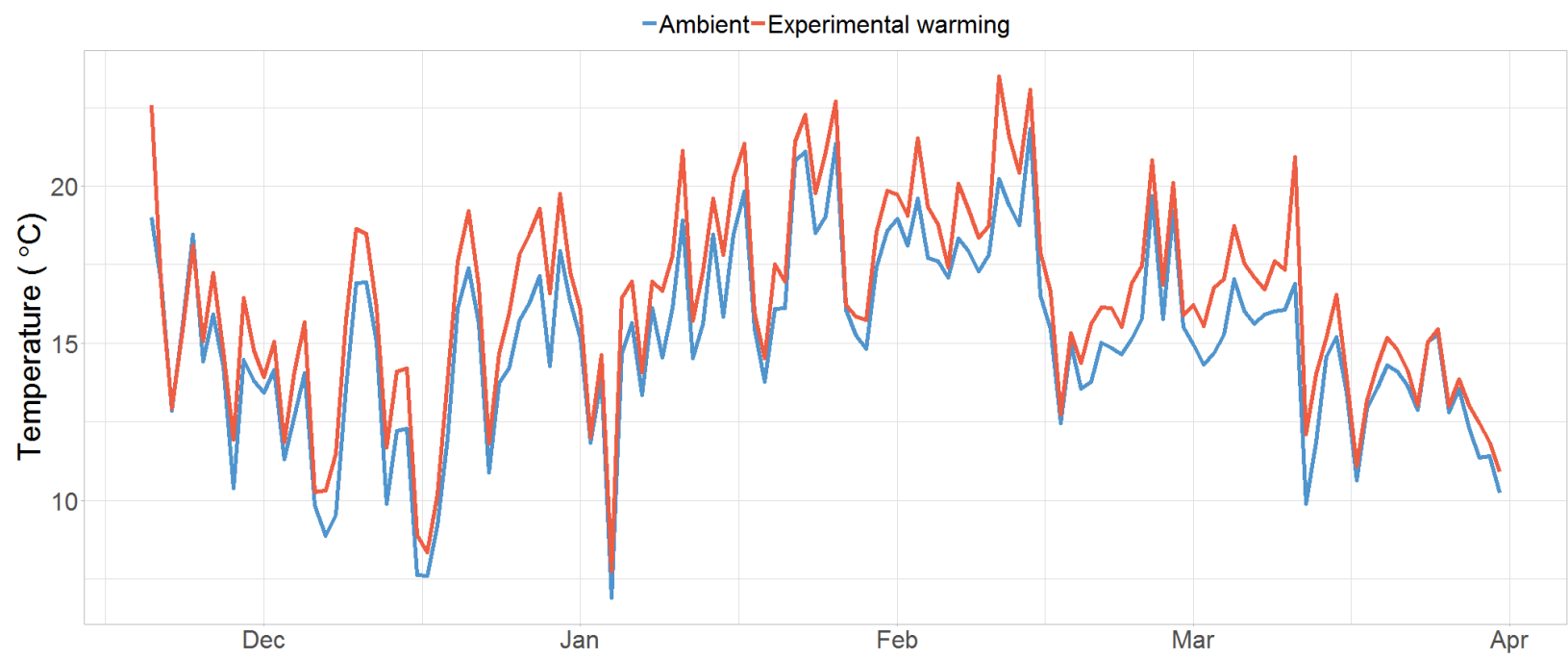

Supplementary Figure 2: Manipulative warming experiment treatment effect: daily mean temperatures $\left({ }^{\circ} \mathrm{C}\right.$ ) in experimentally warmed (red) and ambient temperature (blue) plots over the 2015/2016 austral summer in Tongariro National Park, New Zealand. 


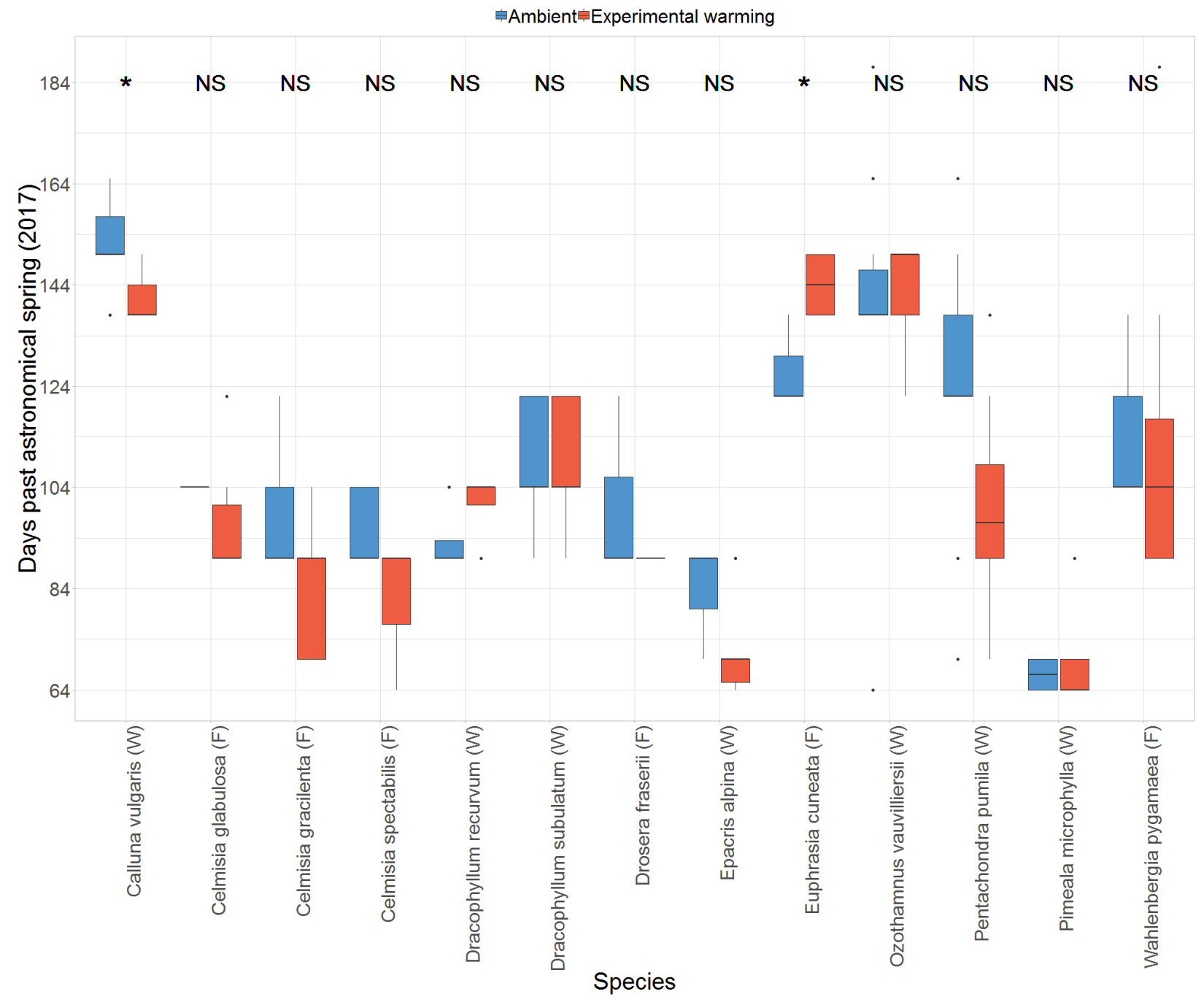

Supplementary Figure 3: First flowering responses of plant species over the 2016/2017 austral summer to experimental warming. Significant differences between experimentally warmed (red) and ambient temperature (blue) plots are indicated by an asterisk. Growth form is denoted in the $X$ axis labels, with woody shrubs and forbs denoted by $W$ and $F$, respectively. 


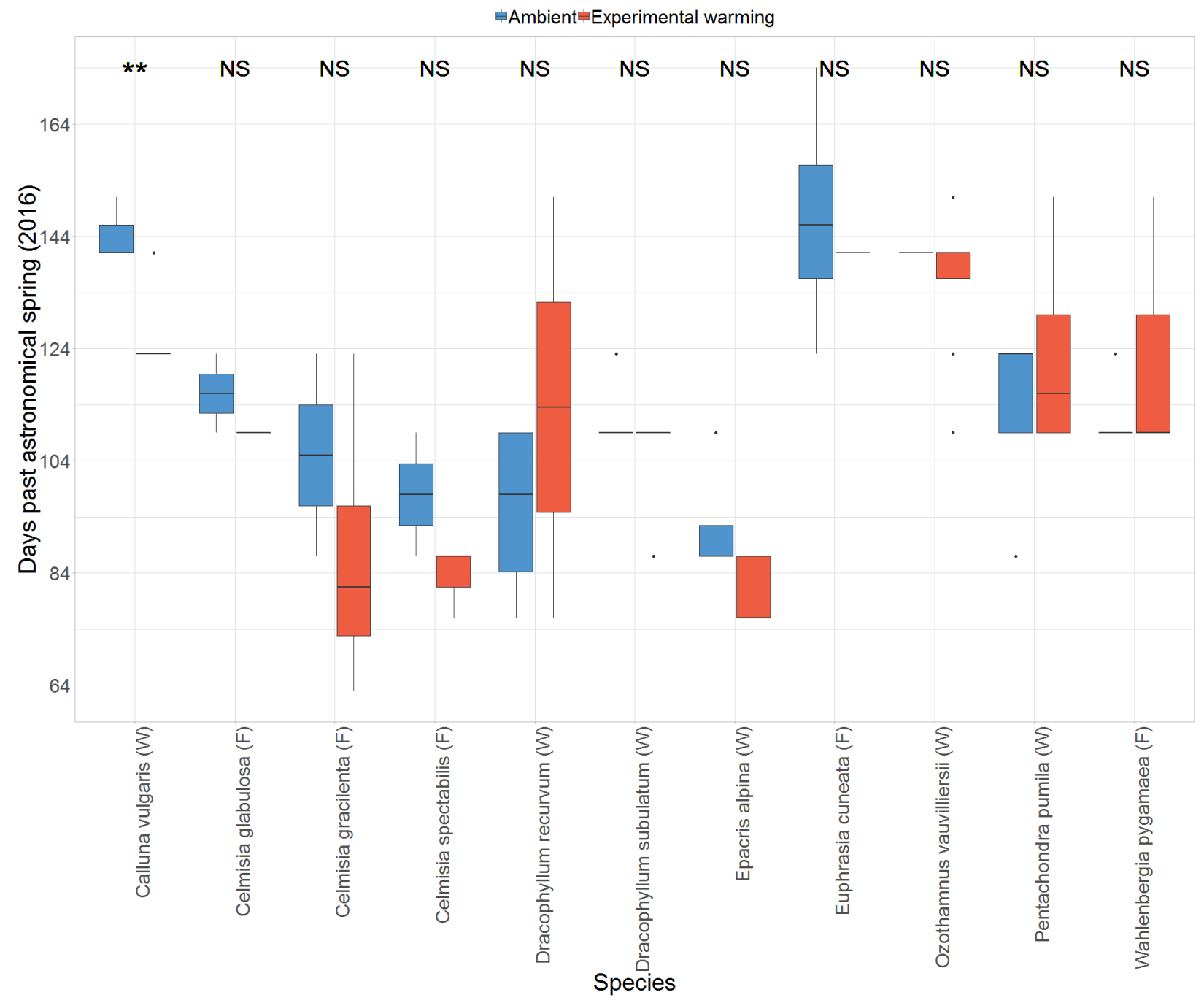

Supplementary Figure 4: First flowering responses of plant species over the 2015/2016 austral summer to experimental warming. Significant differences between experimentally warmed (red) and ambient temperature (blue) plots are indicated by an asterisk. Growth form is denoted in the $X$ axis labels, with woody shrubs and forbs denoted by $W$ and $F$, respectively. 


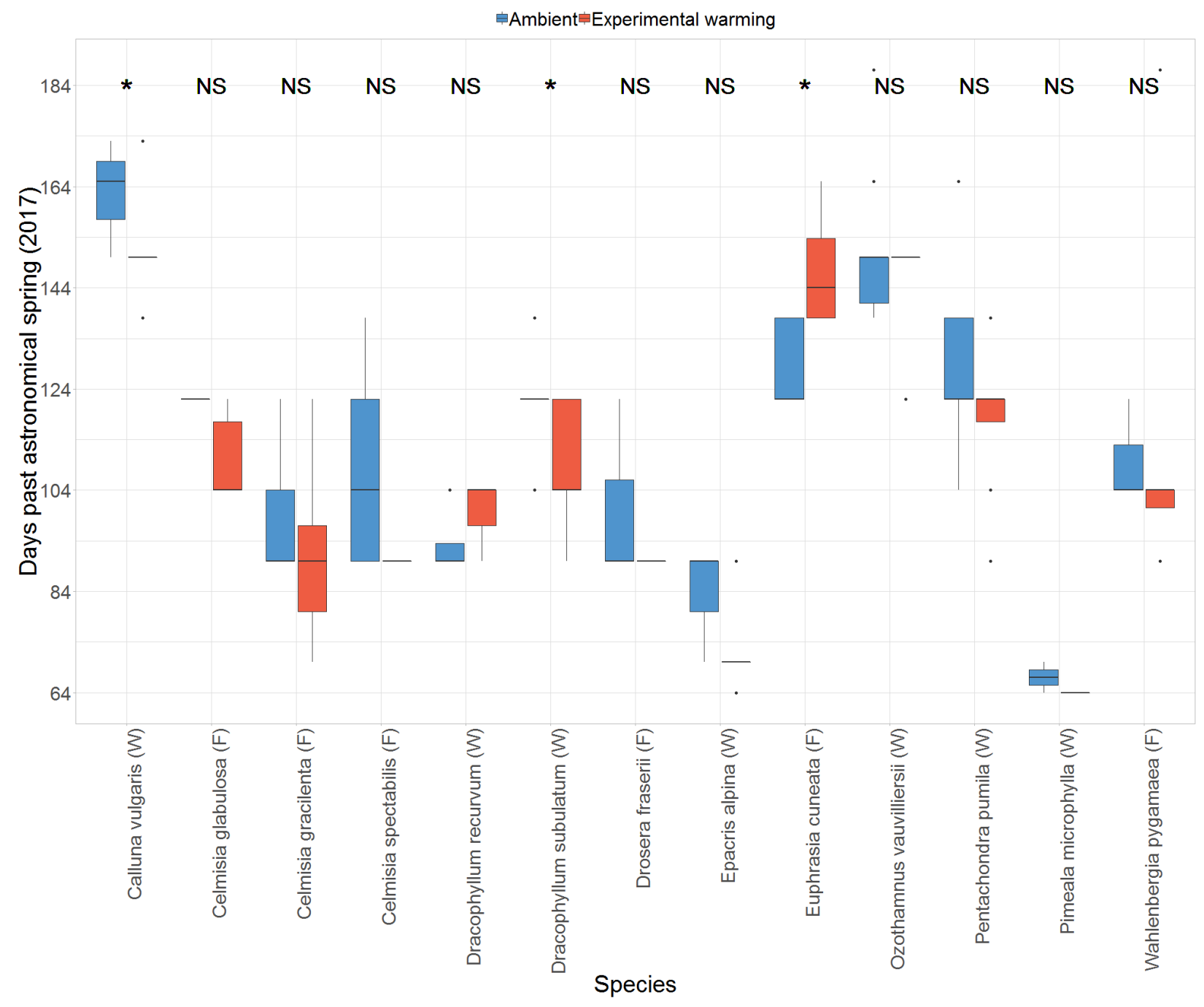

Supplementary Figure 5: Peak floral abundance responses of plant species to experimental warming. Significant differences between experimentally warmed (red) and ambient temperature (blue) plots are indicated by an asterisk. Growth form is denoted in the x-axis labels, with woody shrubs and forbs denoted by $W$ and $F$, respectively. 
自Ambient的林erimental warming

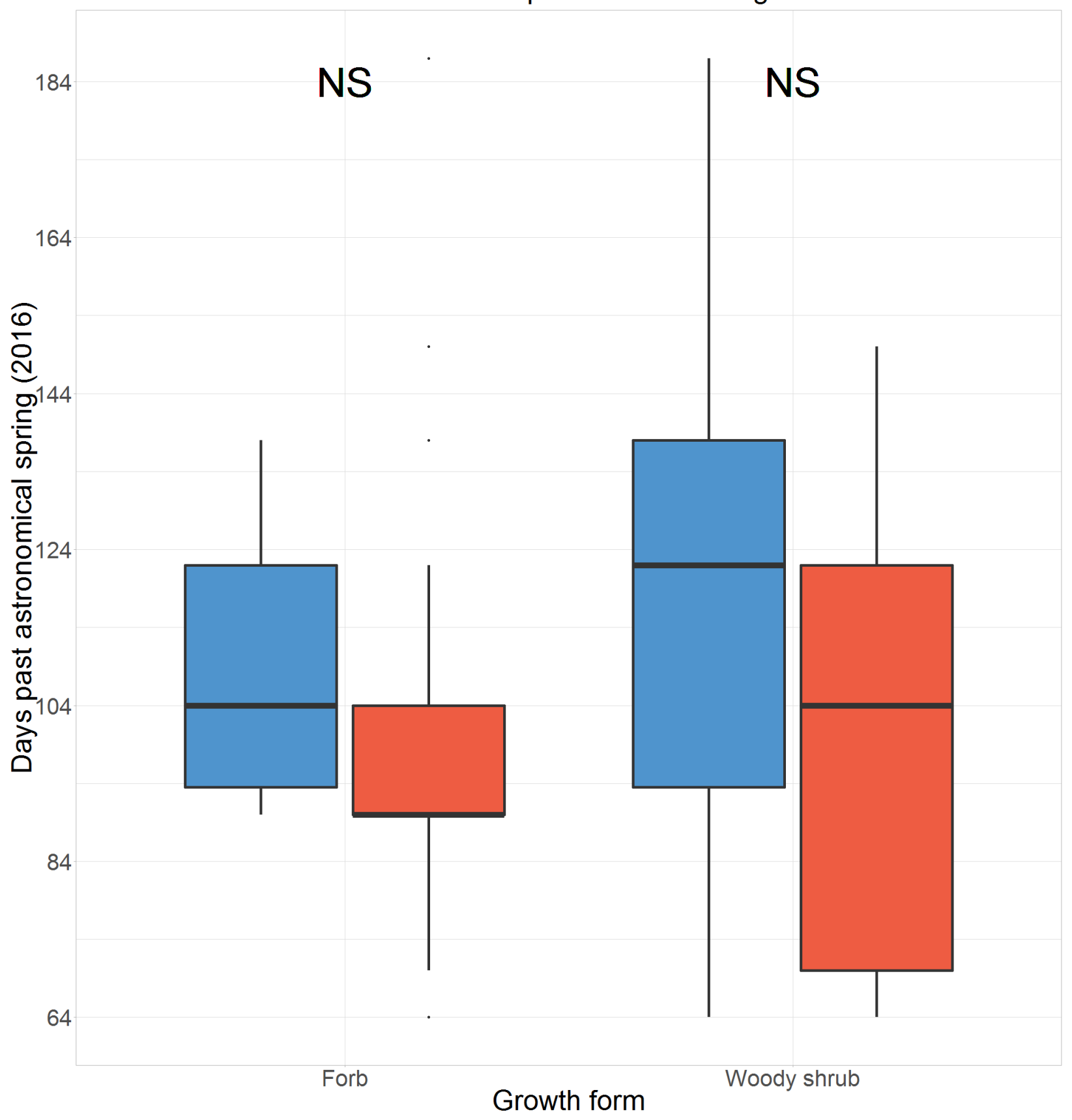

Supplementary Figure 6: First flowering responses by plant growth form over the 2016/2017 austral summer to experimental warming. No significant differences between experimentally warmed (red) and ambient temperature (blue) plots were observed. 


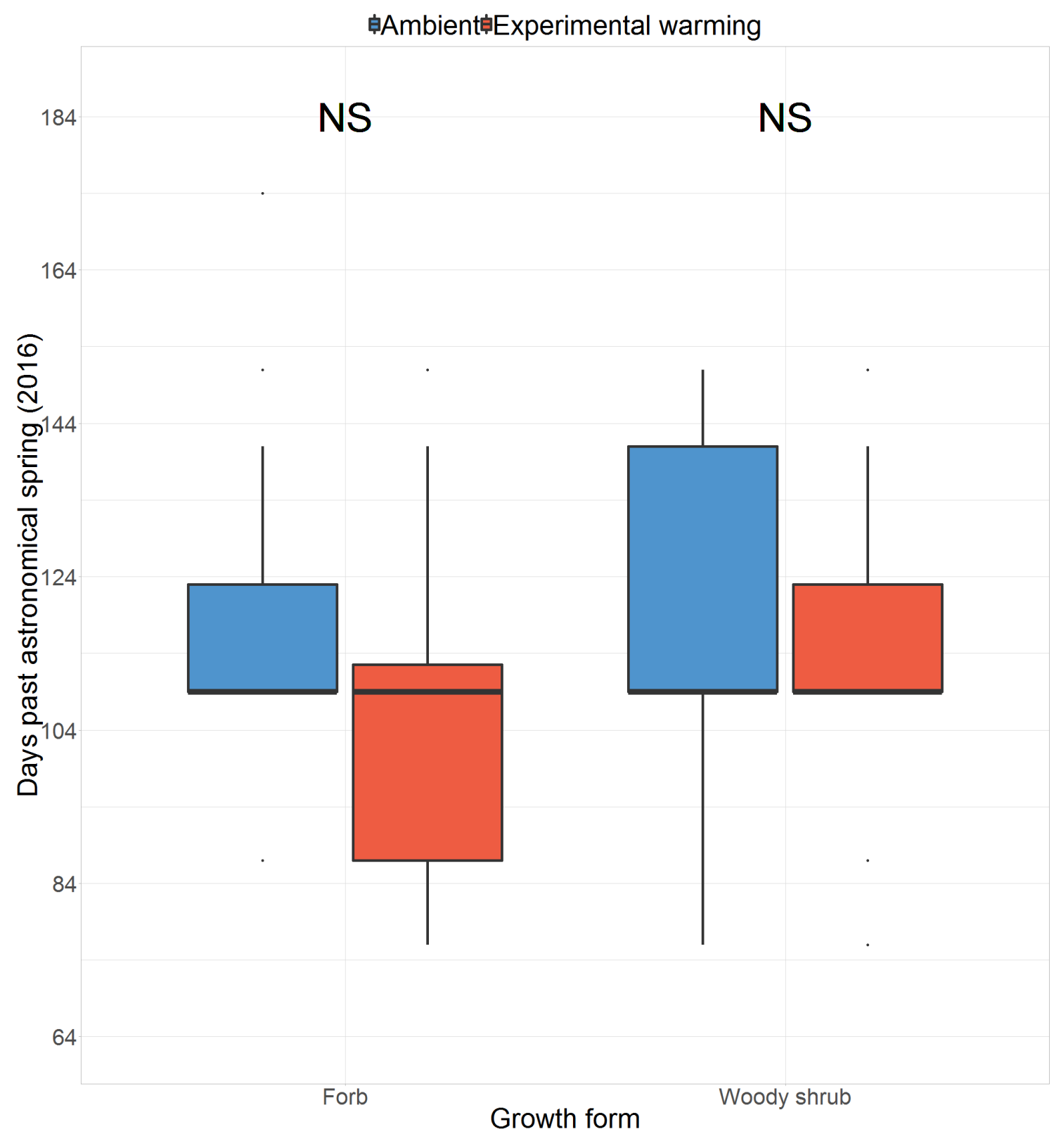

Supplementary Figure 7: First flowering responses by plant growth form over the 2015/2016 austral summer to experimental warming. No significant differences between experimentally warmed (red) and ambient temperature (blue) plots were observed. 
自Ambient淿xperimental warming

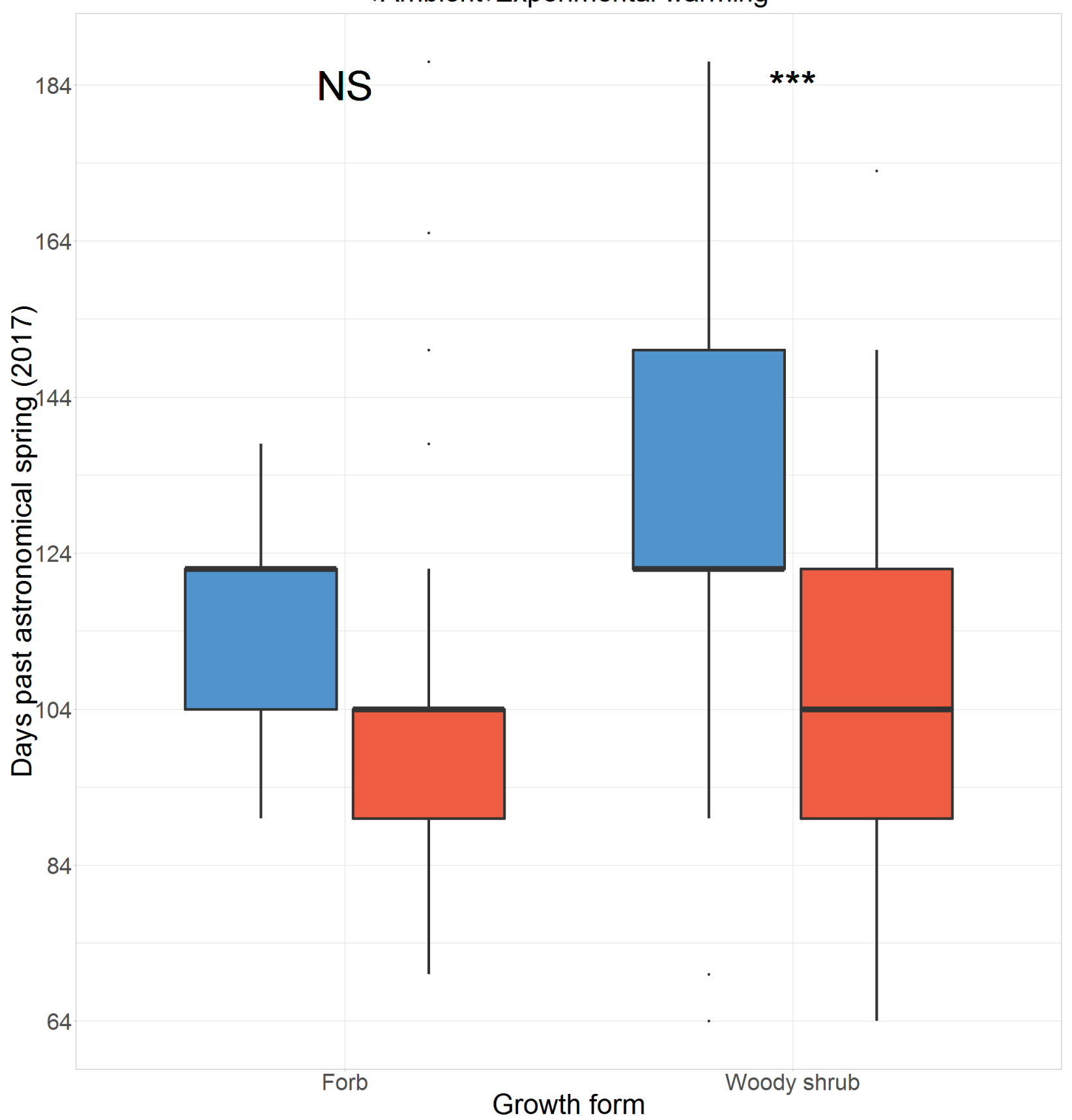

Supplementary Figure 8: Peak floral abundance responses to experimental warming by plant growth form, in experimentally warmed (red) and ambient temperature (blue) plots over the 2016/2017 austral summer. Significant differences are indicated by an asterisk. 


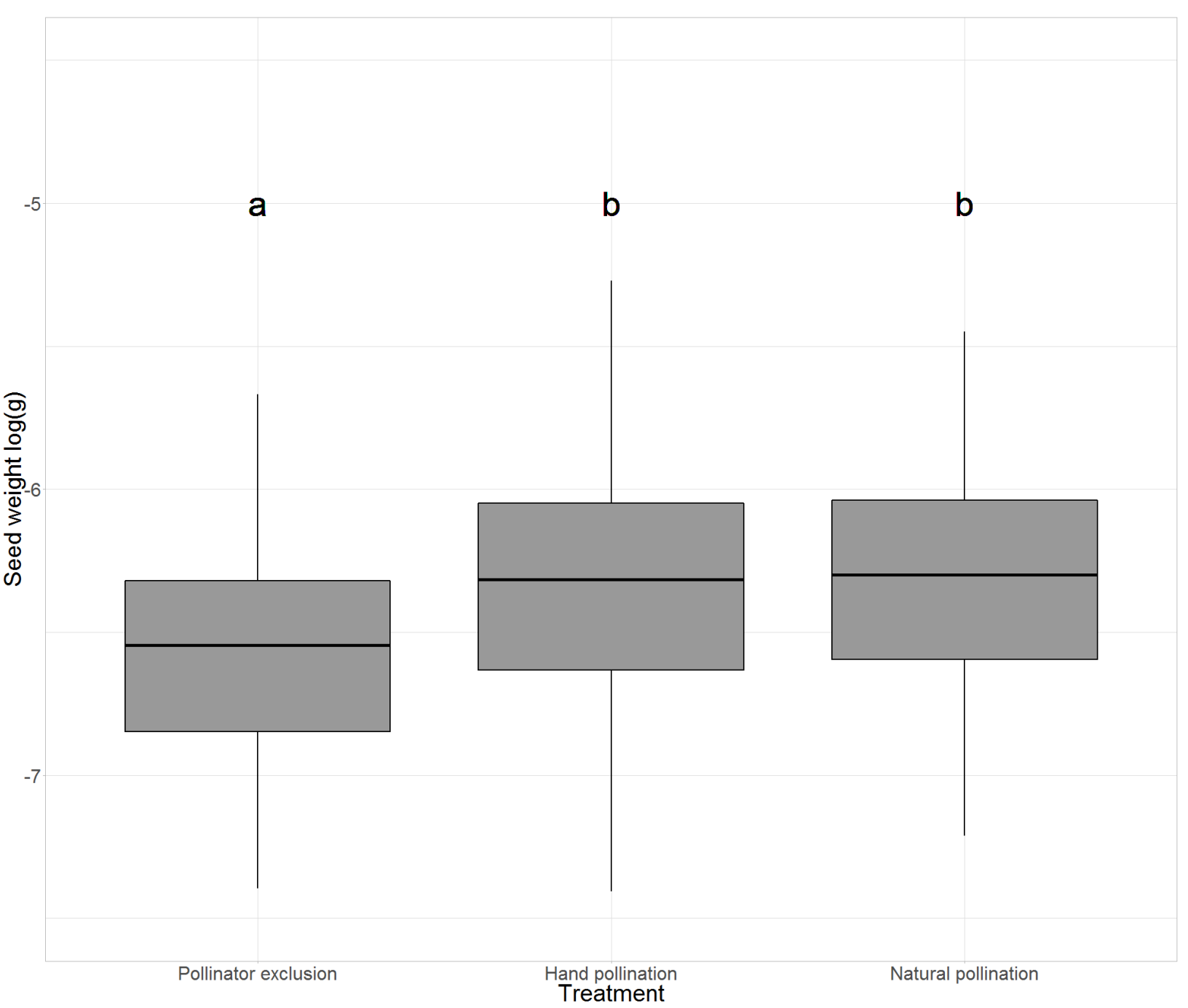

Supplementary Figure 9: Effects of experimental treatment on seed weight of Dracophyllum subulatum. Seed weight is logged and adjusted for the effect of plant height. Letters denote significant differences among treatments. 


\section{Appendix 2: Supplementary materials to Chapter 3 (A)}

Supplementary Table 1: Summary of site-specific linear models predicting Calluna vulgaris biomass $\left(\mathrm{kg} \mathrm{m}^{-2}\right)$ based on canopy cover. All linear models had an intercept fixed at 0 .

\begin{tabular}{|c|c|c|c|c|c|c|}
\hline Im & Plot & $\mathbf{F}$ & DF & DF2 & p & Slope \\
\hline 1 & Broken Fence & 219.7 & 1 & 2 & 0.005 & 5.28 \\
\hline 2 & Cairn & 121.3 & 1 & 4 & $<0.001$ & 10.14 \\
\hline 3 & Carpark Overview & 38.78 & 1 & 3 & 0.008 & 26.17 \\
\hline 4 & Mangatepopo Hut & 64.25 & 1 & 4 & 0.001 & 22.41 \\
\hline 5 & Moss Bowl & 41.53 & 1 & 4 & 0.003 & 10.26 \\
\hline 6 & Old Road & 138.3 & 1 & 3 & 0.001 & 12.44 \\
\hline 7 & Pukeonake Hill & 299.7 & 1 & 3 & $<0.001$ & 37.56 \\
\hline 8 & Scoria Flats & 26.64 & 1 & 3 & 0.014 & 7.91 \\
\hline 9 & Taranaki Falls & 128.2 & 1 & 4 & $<0.001$ & 20.24 \\
\hline 10 & Waihohonu & 41.58 & 1 & 4 & 0.003 & 19.70 \\
\hline 11 & Whakapapa Skifield & 227.7 & 1 & 4 & $<0.001$ & 6.56 \\
\hline 12 & Whakapapanui Stream & 44.99 & 1 & 4 & 0.003 & 12.30 \\
\hline 13 & Whio & 122.9 & 1 & 4 & $<0.001$ & 33.75 \\
\hline 17 & Low Elevation & 165.8 & 1 & 11 & $<0.01$ & 4.57 \\
\hline
\end{tabular}

Supplementary Table 2: The performance of various forms of Generalized Additive Model (GAM) used to predict native alpine plant species richness at a $1 \mathrm{~m}^{2}$ and $5 \mathrm{~m}^{2}$ scales, and the slope ( $z$ ) of the Species Area Relationship. Selected models are highlighted in green.

\begin{tabular}{|c|c|c|c|c|c|c|c|}
\hline \multirow{2}{*}{\multicolumn{2}{|c|}{ GAM }} & \multicolumn{2}{|c|}{ Richness $1 \mathrm{~m}^{2}$} & \multicolumn{2}{|c|}{ Richness $5 \mathrm{~m}^{2}$} & \multicolumn{2}{|c|}{$z$} \\
\hline & & $D^{2}$ & AIC & $D^{2}$ & $\mathrm{AIC}$ & $D^{2}$ & AIC \\
\hline 1 & Elevation & 14.1 & 33.5 & 28.8 & 36.5 & 0.00001 & 127.34 \\
\hline 2 & Invader cover & 17.4 & 36.26 & 19.3 & 33.07 & 11.2 & 130.668 \\
\hline 3 & Invader biomass & 17.1 & 36.01 & 9.35 & 29.87 & 8.16 & 129.73 \\
\hline 4 & Elevation + Invader cover & 27.8 & 45.62 & 33 & 38.16 & 11.2 & 130.667 \\
\hline 5 & Elevation $\mathrm{x}$ Invader cover & 31.3 & 49.17 & 44.3 & 43.25 & 16.7 & 132.446 \\
\hline 6 & Elevation + Invader biomass & 27.2 & 45.07 & 30.3 & 37.1 & 8.16 & 129.73 \\
\hline 7 & Elevation $\mathrm{x}$ Invader biomass & 38.6 & 57 & 48.4 & 45.355 & 9.85 & 130.24 \\
\hline 8 & Elevation $\mathrm{x}$ Invader biomass + Invader cover & 36.2 & 54.28 & 52.2 & 47.48 & 13.8 & 131.499 \\
\hline 9 & Elevation $\mathrm{x}$ Invader cover + Invader Biomass & 39.7 & 58.24 & 44.3 & 43.25 & 16.7 & 132.446 \\
\hline
\end{tabular}




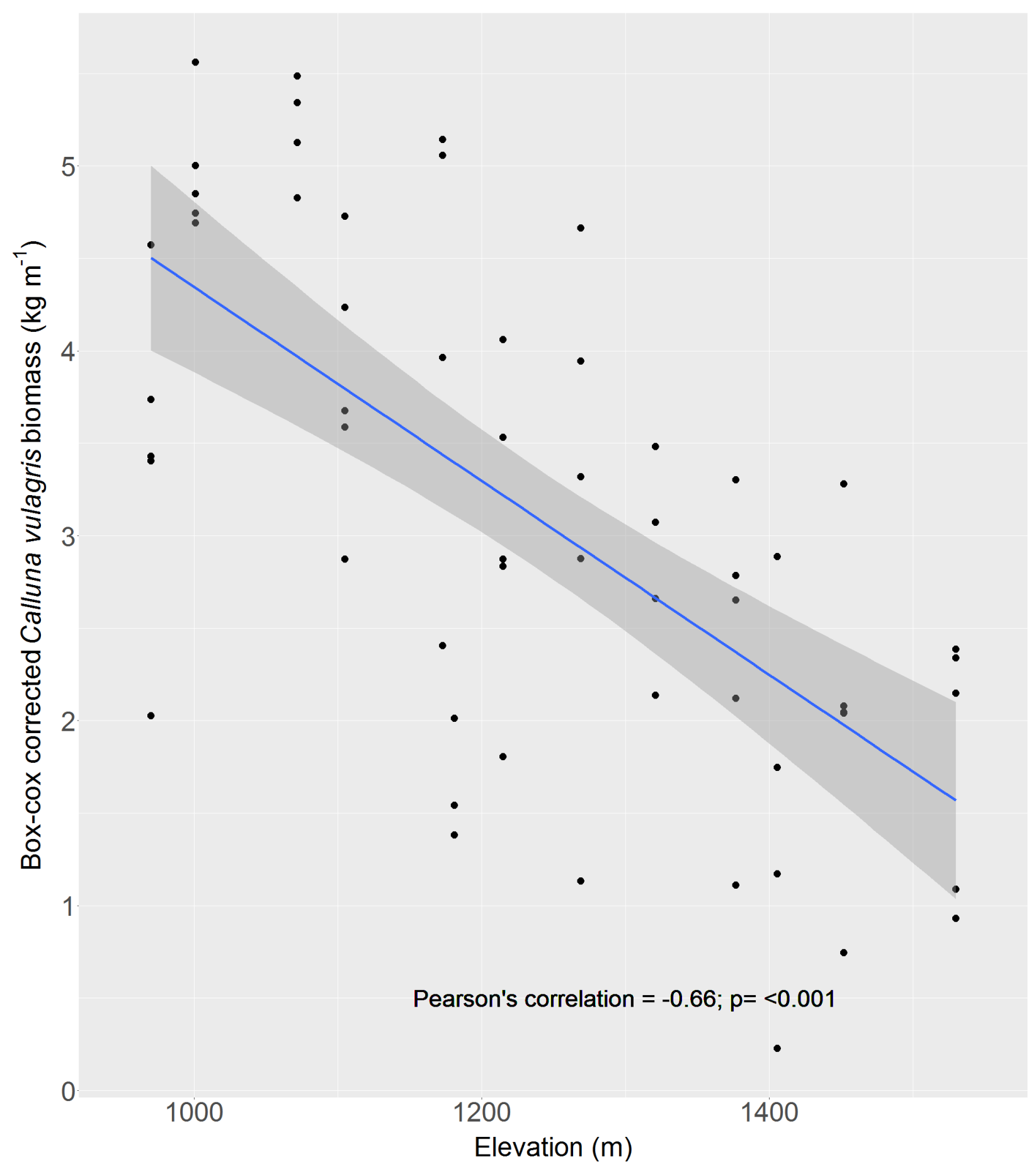

Supplementary Figure 10: Effect of elevation $(\mathrm{m})$ on standardized Calluna vulgaris biomass (kg $\mathrm{m}^{-2}$ ) in Tongariro National Park, New Zealand. Biomass is corrected for heteroskedasticity using a box-cox transformation. 


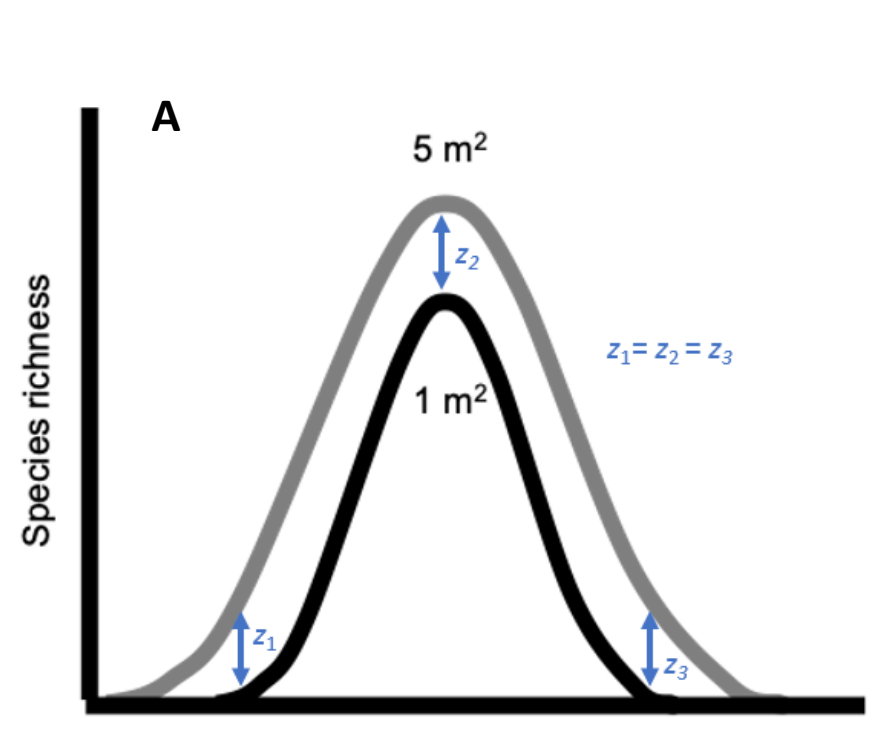

\section{Elevation}
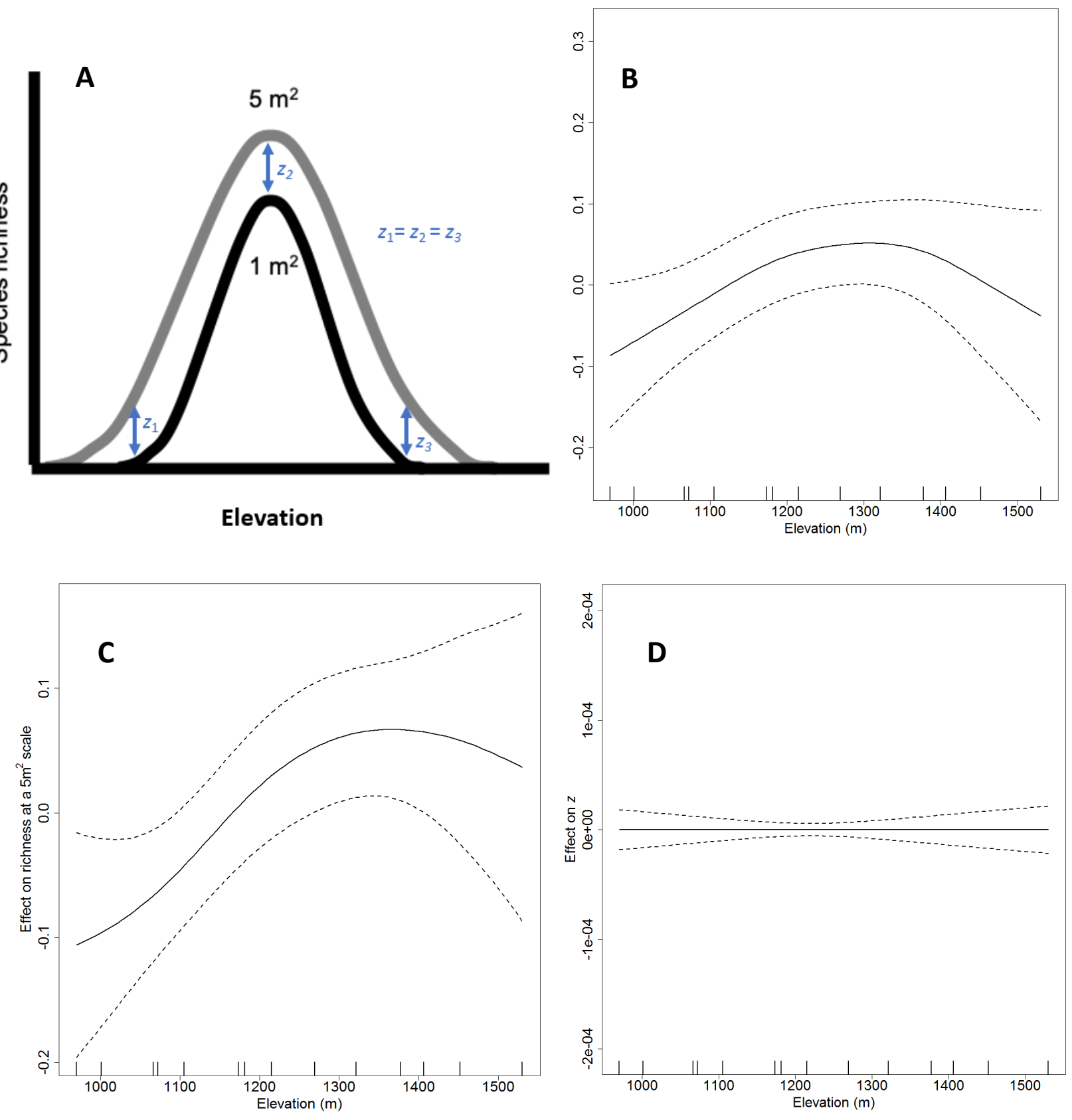

Supplementary Figure 11: Effect of elevation $(\mathrm{m})$ on native alpine plant species richness; A) hypothesized response across spatial scales; predictions fitted by GAM regressions: B) at a $1 \mathrm{~m}^{2}$ scale; C) at a $5 \mathrm{~m}^{2}$ scale; D) on the slope (z) of the Species Area Relationship 


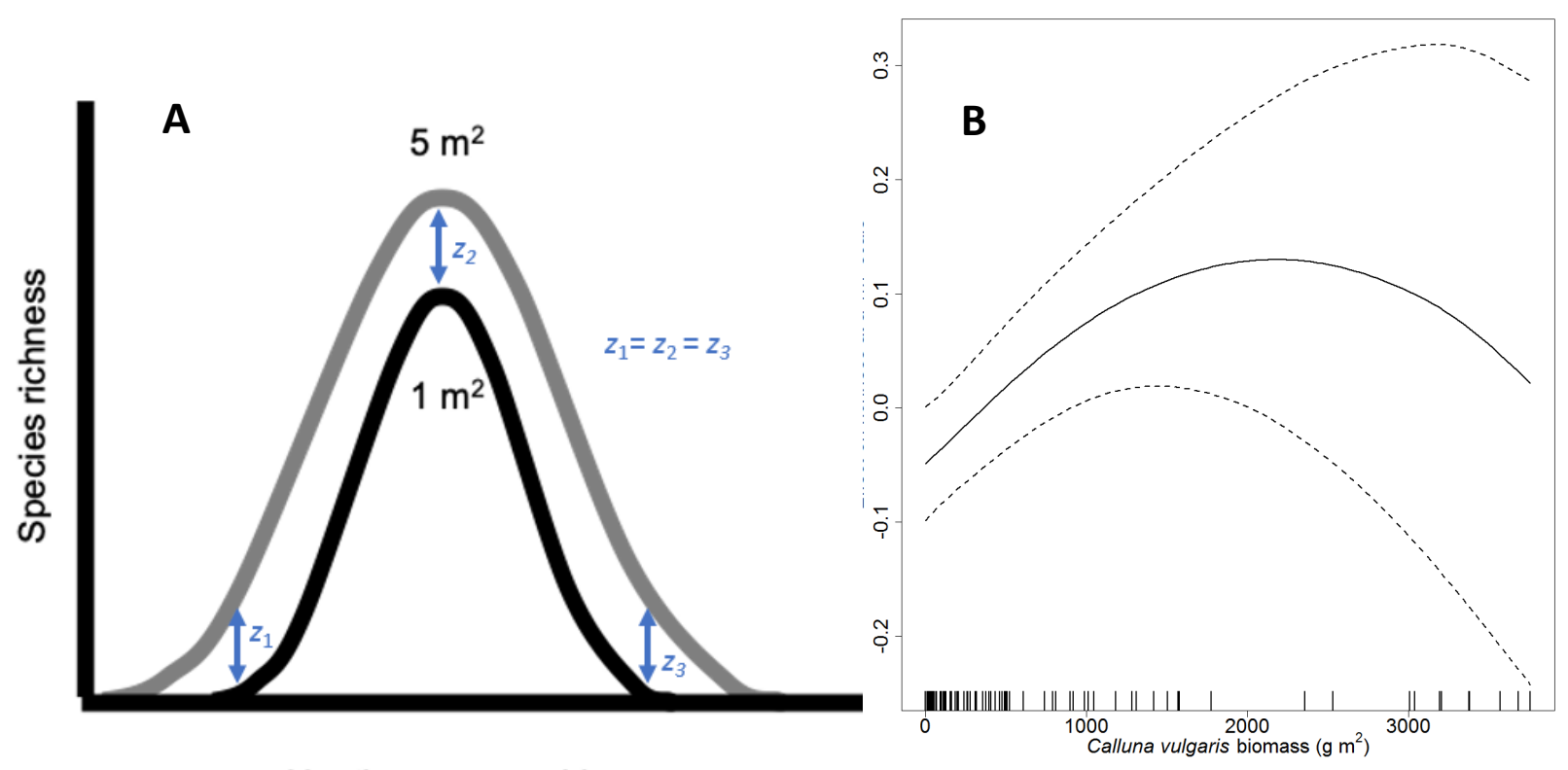

Heather cover or biomass
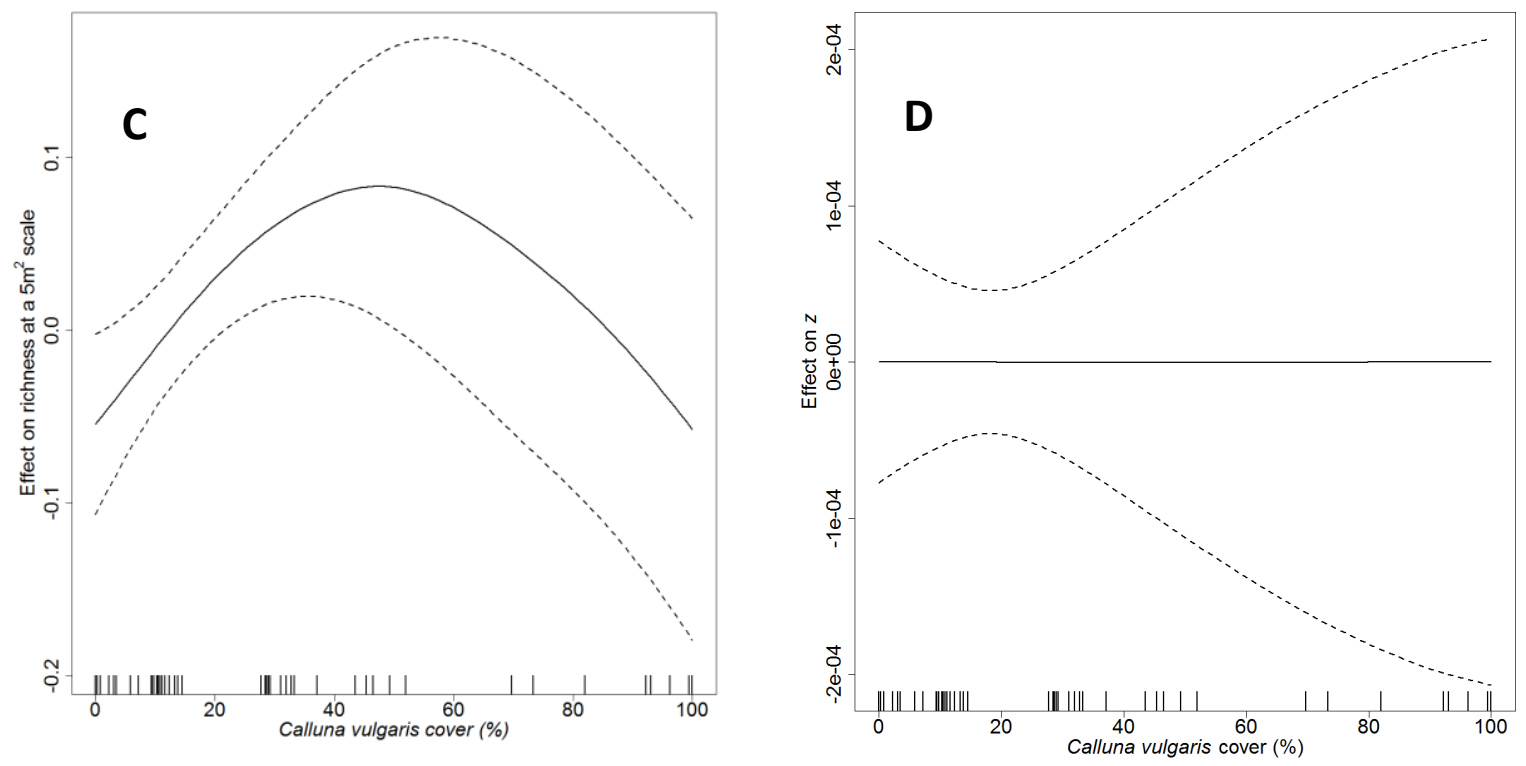

Supplementary Figure 12: Effect of Calluna vulgaris invasion intensity on native alpine plant species richness; A) hypothesized response across spatial scales; predictions fitted by GAM regressions: B) effect of biomass at a $1 \mathrm{~m}^{2}$ scale; C) effect of canopy cover at a $5 \mathrm{~m}^{2}$ scale; D) effect of canopy cover on the slope $(z)$ of the Species Area Relationship. 


\section{Appendix 3: Supplementary materials to Chapter 3 (B)}

\section{Species-specific responses to Calluna vulgaris canopy cover}

I presented a version of this work at an international conference in 2018:

Giejsztowt J., Classen, AT. \& Deslippe JR. Climate change and weed invasion synergistically affect species richness in an alpine plant community. Society for Conservation Biology-5th Oceana Congress. Wellington, New Zealand, 2-6 July 2018.

\section{Rationale}

Previous work suggests that some native plant species in Tongariro National Park are particularly vulnerable to, or favoured by, Calluna vulgaris invasion (Rogers and Leathwick 1996). The aim of this body of work was to investigate species-specific responses to $C$. vulgaris invasion. We intended to identify species which were particularly negatively affected by $C$. vulgaris invasion.

\section{Methodology and Results}

This analysis was carried out in a two-step process. First, we investigated the relationship between $C$. vulgaris canopy cover and species richness. We identified tipping points of $C$. vulgaris cover at which species richness was significantly reduced. Secondly, we ran a species indicator analysis on the partitions identified in the first step, to see whether species richness reductions were associated with non-random species loss.

In the first step, the dataset, collected as per the methodology in Chapter 3 was limited to include only plots within one elevational band, 1066-1181 m a.s.l. to exclude the effects of elevational gradients. Further, plots with no $C$. vulgaris were excluded. Finally, we limited the dataset to only $1 \mathrm{~m}^{2}$ plots. 60 species were represented in the final dataset. The reduced dataset $(n=81)$ was analysed using regression tree analysis in the 'rpart' package (Therneau and Atkinson 2018) in R (R Development Core Team 2016). This technique is a method for the identification of tipping points (Elith et al. 2008). We identified two tipping points in the dataset: at 92.5 and 55\% C. vulgaris cover (Supplementary Figure 13A and B). The model was 
associated with significant reductions in variance in the dataset (the relative error for a tree with two splits was $65 \%$ of total error). The viability of these tipping points was further supported with Wilcoxon-signed rank tests using the function 'wilcox.test'. This test supported plots within the partition at $55 \%$ C. vulgaris cover. The partition at $92.5 \%$ had too few data points above the threshold for the Wilcoxon-signed rank test to be reliable. The mean number of species in the highest $C$. vulgaris cover partition ( $x>92.5 \%$ ) was 4 , in the intermediate (55$92.5 \%)$ C. vulgaris cover partition was 8.9 and in the low C. vulgaris partition (<55\%) was 11.2. In the second step, we used a more comprehensive version of the dataset provided in Chapter 3, containing the canopy cover (\%) of all species. We used the same selection of $1 \mathrm{~m}^{2}$ quadrats as above. We assigned the plots to each of the three partitions based on $C$. vulgaris canopy cover within that plot and ran a species indicator analysis using the package 'indicspecies' (De Caceres and Legendre 2009). We found numerous species which were significantly associated with one or two of the partitions.

Supplementary Figure 13: Species specific responses to Calluna vulgaris invasion (Next page); A) form of the regression tree describing native alpine plant richness responses to $C$. vulgaris canopy cover; B) plot of richness as a function of $C$. vulgaris cover, with regression tree thresholds indicated; $\mathbf{C}$ ) table of results of the species indicator species analysis, displaying species which are particularly associated with low or intermediate levels of $C$. vulgaris cover. 


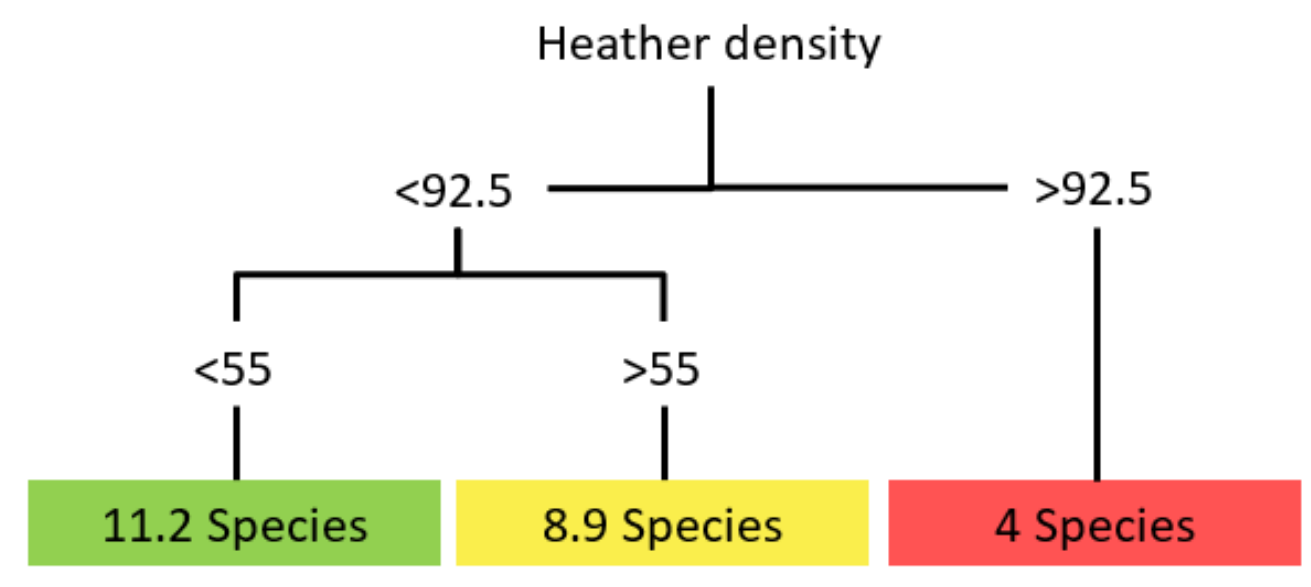

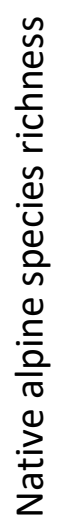

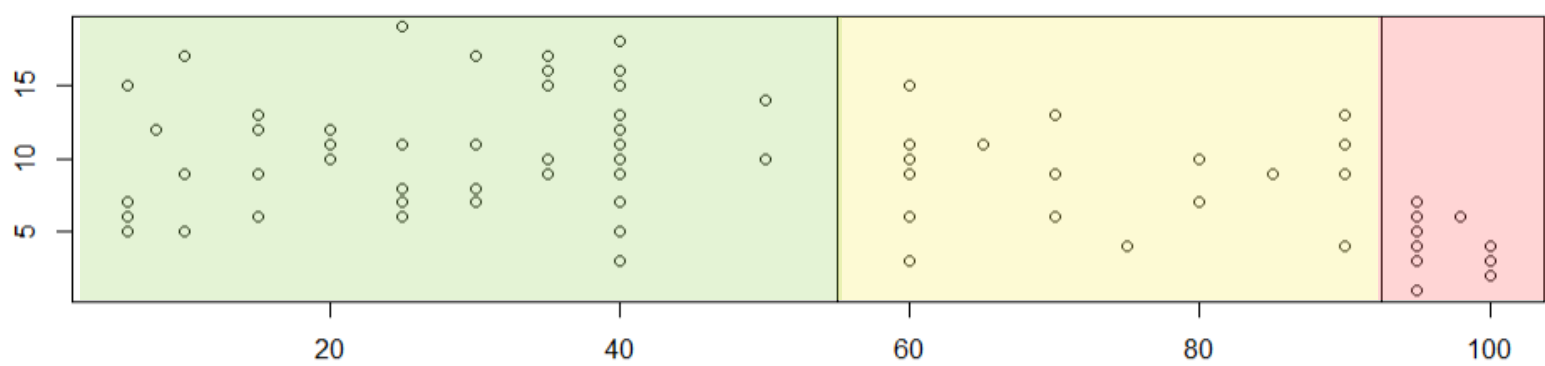

Calluna vulgaris (\% cover)

C Is most commonly found in

Low C. vulgaris density

Intermediate $C$. vulgaris density

Low and intermediate $C$. vulgaris density
Species

Dracophyllum recurvum

Epacris alpina

Anisotome aromatica

Leptospermum scoparium

Gaultheria depressa

Rytidiosperma gracilenta

Gaultheria macrostigma

Chionochloa rubra

Pentachondra pumila

Wahlenbergia pygmaea

Celmisia gracilenta
Specificity Sensitivity Stat $p$

\begin{tabular}{l|l|}
\hline 0.98 & 0.61 \\
\hline
\end{tabular}

$0.780 .001 * * *$

0.85

0.71

$0.780 .001 * * *$

0.83

0.360 .540 .045 *

0.65

0.79

$0.720 .003^{* *}$

0.86

0.43

$0.610 .003^{* *}$

0.70

0.50

$0.590 .007^{* *}$

0.92

$0.210 .450 .029 *$

0.98

0.71

$0.840 .002 * *$

0.97

0.64

$0.790 .004^{* *}$

1

$0.430 .660 .024 *$

1

$0.38 \quad 0.610 .035 *$ 


\section{The Future of Our Taonga Tipu}

Modelling the vulnerability of culturally important environmental change

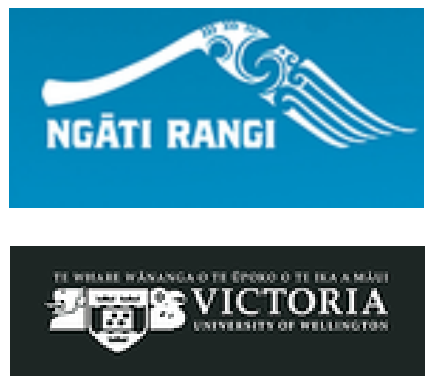

Report prepared for Ngāti Rangi and the Department of Conservation by Justyna Giejsztowt and Dr Julie Deslippe Centre for Biodiversity and Restoration Ecology, School of Biological Sciences, Victoria University of Wellington

\section{Executive Summary}

- Drivers of environmental change, such as climate change and species invasions, can alter the distribution and density of native plant species, including those that are culturally important to Māori (taonga tipu).

- Species Distribution Models (SDMs) are powerful tools for predicting the impact of climate change on plant species distributions, but they typically omit the effects of species interactions and rarely incorporate cultural interests.

- Tongariro National Park (TNP) is a UNESCO natural and cultural World Heritage Site that is subject to a changing climate and the invasion of European heather (Calluna vulgaris (L.) Hull).

- In collaboration with Ngāti Rangi, we gathered mātauranga Māori via interviews and identified five taonga tipu.

- We surveyed taonga tipu and C. vulgaris in TNP in $4901 \mathrm{~m}^{2}$ plots set along elevational and invasion gradients. Data from field surveys was further bolstered with publicly available occurrence data to underpin robust SDMs of the climatic tolerances of each species.

- We parameterized pairwise competition between $C$. vulgaris and each taonga tipu using Boosted Regression Trees (BRT) of plant densities along the invasion gradient. 
- We then parameterized SDMs of taonga tipu based on both predicted climate scenarios and predicted $C$. vulgaris distribution and density in those climates.

- We found that future climates will facilitate both an expansion of the distribution of $C$. vulgaris and increasing densities of $C$. vulgaris in TNP.

- We found these responses of $C$. vulgaris will lead to reductions in the distribution and densities of three of the five taonga tipu modelled.

- Further, our models predict no stable or increasing areas of suitable habitat in the future for any of the five taonga tipu studied.

- We conclude that the interacting drivers of global change can act synergistically to cause greater reductions in suitable habitats for endemic plant species in TNP. Importantly, these effects will be most negative for species that are vulnerable to both climate change and weed invasion.

- In the future the conservation of native alpine plants is likely to become an even greater challenge. We suggest that the conservation prioritization process urgently consider the effects of the interacting drivers of global change for successful kaitiakitanga of our taonga tipu.

\section{Aim}

To understand how multiple agents of global environmental change (i.e. climate change, weed invasion) may combine to affect the future distributions of suitable habitats for taonga tipu in Tongariro National Park.

\section{Methodology}

\section{Species selection}

Deborah Te Riaki led interviews with pāhake and others with mōhiotanga pertaining to taonga tipu. The interview questionnaire and associated documents were approved by the Victoria Universities' Human Ethics Committee. Deb selected interviewees and conducted interviews. Six interviews were transcribed and summarised in a report. With written consent of the interviewees, we stored the interview recordings in Ngāti Rangi archives. 
We created a list of all the plant species mentioned explicitly in the interviews and those which provided ecosystem services which were important to interviewees. A subset of these species was associated with alpine environments for which we held preliminary data. We selected plant species for modelling based on three criteria. Firstly, we were interested in species which were identified as taonga through the interview process. We prioritised species which were explicitly mentioned by interviewees and/or if they contribute substantially to an ecosystem service that was identified as important to the interviewee. Secondly, we chose species which, based on our preliminary data, appeared vulnerable to either climate change (e.g. strongly associated with cooler climates) or heather invasion, or both. Thirdly, to ensure that our analyses could apply to the widest range of plant species, we selected plants with a range of vulnerabilities to heather and climate and with different growth forms. The species selected for modelling and the rationale for selecting each is shown in

\section{Supplementary Table 3.}

Supplementary Table 3: Rationale for selecting five taonga tipu for modelling in this study.

\begin{tabular}{|c|c|c|c|c|}
\hline & \multirow{2}{*}{ Species } & \multicolumn{2}{|c|}{ Vulnerability to threat } & \multirow{2}{*}{ Evidence of taonga status } \\
\hline & & Climate change & Heather & \\
\hline & $\begin{array}{l}\text { Gentian } \\
\text { Gentianella bellidifolia } \\
\text { Forb }\end{array}$ & High & Moderate & Explicitly mentioned by iwi \\
\hline & $\begin{array}{l}\text { Red tussock } \\
\text { Chionochloa rubra } \\
\text { Tussock grass }\end{array}$ & Moderate & Moderate & $\begin{array}{l}\text { Ecosystem structure } \\
\text { important to iwi }\end{array}$ \\
\hline & $\begin{array}{l}\text { Creeping coprosma } \\
\text { Coprosma perpusilla } \\
\text { Dwarf woody shrub }\end{array}$ & Moderate & High & $\begin{array}{l}\text { Biodiversity and ecosystem } \\
\text { structure important to iwi }\end{array}$ \\
\hline & $\begin{array}{l}\text { Neinei } \\
\text { Dracophyllum recurvum } \\
\text { Woody shrub }\end{array}$ & High & Moderate & $\begin{array}{c}\text { Biodiversity and ecosystem } \\
\text { structure important to iwi. } \\
\text { This species has as a Maori } \\
\text { name }\end{array}$ \\
\hline incher & $\begin{array}{l}\text { Kopoti } \\
\text { Anisotome aromatica } \\
\text { Forb }\end{array}$ & Low & Moderate & $\begin{array}{l}\text { Biodiversity important to iwi. } \\
\text { This species has as a Maori } \\
\text { name }\end{array}$ \\
\hline
\end{tabular}

\section{Modelling Climatic Envelopes}

\section{Data Sources}


We predicted the potential distributions of $C$. vulgaris and the five taonga tipu under the current climate and two future climate scenarios. Future climate projections were made for the year 2070. We chose to indicate possible future scenarios with two Representative Concentration Pathways (RCP): 4.5 (an optimistic climate change scenario) and 8.5 (a businessas-usual climate change scenario), as dependent on political and socio-economic adaptation to the climate change threat.

"BioClim" raster layers (WorldClim.org) are a set of 19 environmental layers based on observed data and future climate data based on global circulation model predictions; these were downloaded at a 30 arcsecond resolution. "Current conditions" are interpolations of observed data from the years 1960-1990. "Future climatic conditions" were created from "BioClim" raster layers (WorldClim.org) downloaded at a 30 arcsecond resolution. Our future conditions were based on five Global Circulation Models (GCMs) chosen from among the forty models used in the Intergovernmental Panel on Climate Change (IPCC) Fifth Assessment. Model choice was based on the ability of GCMs to validate accurately on present day data, as well as being as different as possible to one another in terms of projections (as verified by The National Institute of Water and Atmospheric Research; NIWA). GCMs selected were HadGEM2-ES (MOHC: UK), GISS-E2-R (NASA-GISS: USA), GFDL-CM3 (NOAA-GFDL: USA), BCC-CSM1.1 (BCC: China) and NorESM1-M (NCC: Norway). The climatic predictions from each of the five GCMs were averaged within each RCP scenario.

\section{Range}

The North Island of New Zealand includes many areas which are under intensive productive management; such landscapes typically have low native plant biodiversity. As this management is particularly prolific in lowland areas, inclusion of these areas within the calibration range of the models lead to significant error. Therefore, model calibration for taonga tipu was carried out within Tongariro National Park (TNP) and several other protected areas on the North Island (Awarua Conservation Area, Egmont National Park, Kaimanawa Forest Park, Kaweka Forest Park, Ruahine Forest Park and Tararua Forest Park; Supplementary Figure 14). Boundaries of calibration ranges were based on the Protected Area Layer, which is derived from the Landonline Primary Parcel(s) and was retrieved from data.linz.govt.nz in May 2018. Climate 
data were cropped to the extent calibration using ArcMap 10.4.1. A three-kilometre buffer area outside of these protected areas was included as some vegetation data were collected in vegetatively similar regions on conservation area peripheries. The climatic envelope of $C$. vulgaris was calibrated in New Zealand within TNP only as $C$. vulgaris has not yet reached climatic equilibrium in New Zealand outside of this park. The $C$. vulgaris model was additionally calibrated across a subset of the native range of $C$. vulgaris in Europe which is climatically analogous to New Zealand under present day and predicted future conditions. There were substantially fewer observations in New Zealand than in Europe, therefore European observations were down-weighted.

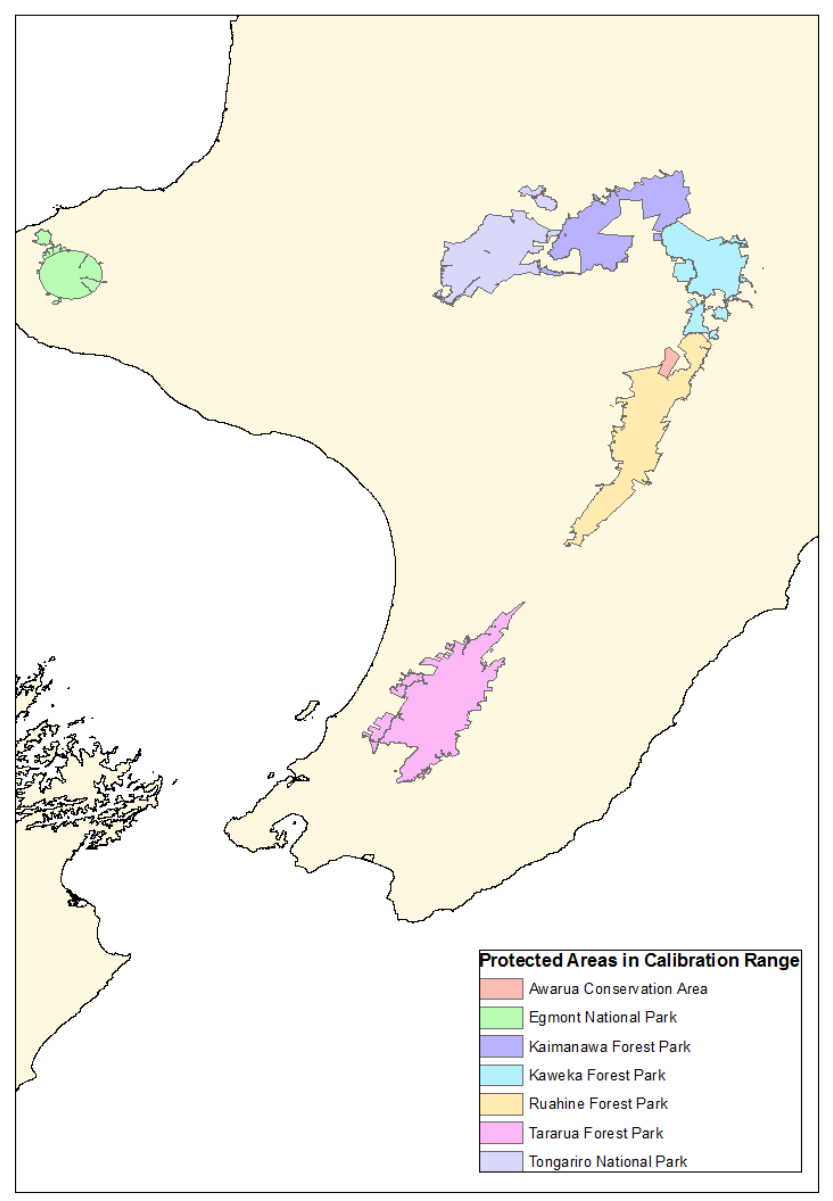

Supplementary Figure 14: Boundaries of seven protected areas used to calibrate taonga tipu climate envelope models. The Calluna vulgaris climate envelope model was calibrated within Tongariro National Park and Europe. 


\section{Predictor variable selection}

Predictor variables were selected based on a two-step process. In the first step, variables with low variability over the calibration range were excluded. In the second stage, collinearity amongst predictor variables was minimised. We achieved both steps by using Principal Component Analysis (PCA). Several predictors were excluded based on low variability over the calibration range (Supplementary Figure 15, indicated by *). For taonga tipu, the remaining predictors clustered into three distinct groups of collinear variables (Supplementary Figure 15A). These clusters corresponded to:

- Absolute temperature

- Temperature variability

- Precipitation rates

For C. vulgaris, remaining predictors clustered into two groups (Supplementary Figure 15B):

- Temperature variability

- Absolute temperature and precipitation rates (which were inversely correlated).

Finally, variables were excluded based on their importance in explaining patterns of occurrences for each of the modelled species. We ran a series of preliminary models for each species, where we initially included all sufficiently variable predictors and excluded one predictor from each grouping stepwise. At each step we excluded a factor from each grouping which had the lowest overall importance in explaining the occurrence patterns of each species. For many species, we removed the final predictor in a grouping if it had very poor explanatory power. 

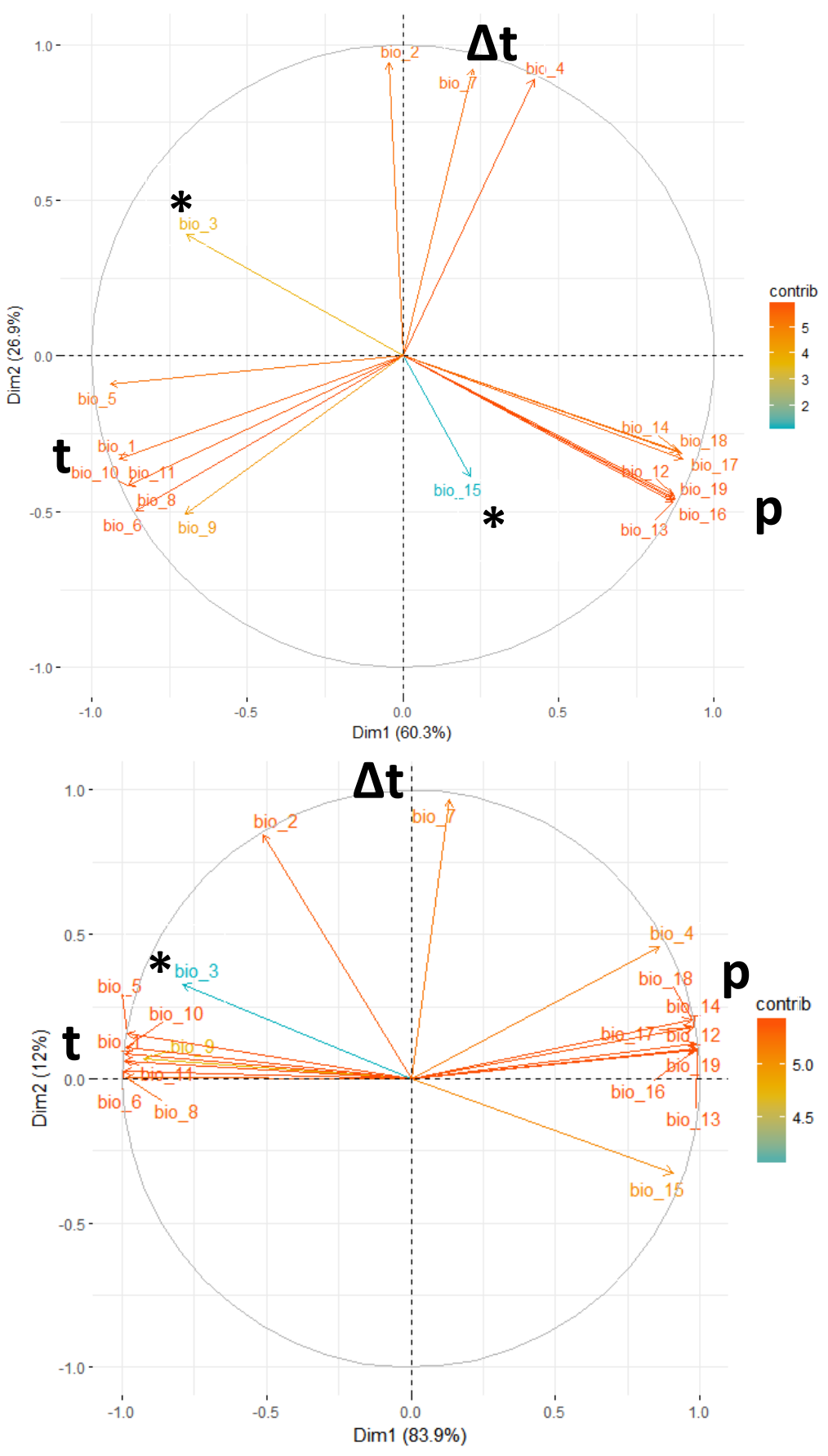

Supplementary Figure 15: Principal Component Analysis of WorldClim "bio" predictor variables for (A) seven protected areas on the North Island of New Zealand (the calibration range for taonga tipu) and (B) Tongariro National Park (the calibration range for $C$. vulgaris). Predictors with low overall variability over each calibration range were excluded and are indicated by * Remaining predictors were in groupings which are indicated by $\mathrm{t}$ (absolute temperature), $\Delta \mathrm{t}$ (temperature variability) and $\mathrm{p}$ (precipitation). 


\section{Pseudo-absence selection}

Ten sets of environmentally stratified pseudo-absences were selected within the calibration range for each species using the BIOMOD2 package in $\mathrm{R}$. As the calibration range was relatively small for all species, the number of pseudo-absences generated was equal to the number of presences used to calibrate the model in each case.

\section{Model algorithm selection}

Models were calibrated with a wide variety of algorithms to identify central tendencies in predictions. The algorithms used were Artificial Neural Network (ANN), Generalized Additive Model (GAM), Generalized Linear Model (GLM), Multiple Adaptive Regression Splines (MARS), Random Forest (RF) and Surface Range Envelope (SRE). Each algorithm was run thrice on a random 80:20 subset of each of the ten pseudo-absence datasets; an additional full model was run on each dataset. This resulted in 240 individual models for each species.

\section{Model validation}

The twenty percent of data excluded from the calibration dataset was used for model validation (sympatric testing); this was carried out using the Receiver Operating Characteristic curve (ROC), with ROC > 0.7 considered adequate performance. Additionally, where possible, models were tested on entirely novel data within an independent geographic range (allopatric testing) and those with a sensitivity of over $70 \%$ considered adequately performing. The independent testing dataset included bias-corrected (protocol as above) occurrence records on the South Island but was limited to only geographic areas which were climatically represented within the training range of each model. This precluded model extrapolation into novel climates where it is expected that models may under-perform.

\section{Ensemble model building}

Individual models which satisfied testing criteria were included in the final model ensemble. These were combined by mean and weighted according to validation success, using ROC sympatric testing scores. We transformed probabilistic ensemble model distribution projections of each taonga tipu and $C$. vulgaris under the current climate into binary predictions. We used the 'roc' function in the package 'pROC' in R to build a ROC curve with probabilistic predictions 
at each of our field survey points as the predictor and true presence/absence data from our field surveys as the response variable. We chose thresholds from the 'local maximas' provided by pROC, prioritizing sensitivity. The ensemble models of climate prepared for $C$. vulgaris and each of the five taonga tipu performed very well, with high ensemble model ROC scores and with high values of sensitivity and specificity (Supplementary Table 4).

Supplementary Table 4: Model performance for each species.

\begin{tabular}{|c|c|c|c|c|c|}
\hline Species & $\begin{array}{l}\text { Calibration } \\
\text { dataset }(n)\end{array}$ & $\begin{array}{c}\text { ROC of } \\
\text { ensemble }\end{array}$ & Sensitivity & Specificity & $\begin{array}{c}\text { Number of models } \\
\text { in ensemble }\end{array}$ \\
\hline $\begin{array}{c}\text { Calluna } \\
\text { vulgaris }\end{array}$ & 10,575 & 0.934 & 94.768 & 99.689 & 239 \\
\hline $\begin{array}{l}\text { Anisotome } \\
\text { aromatica }\end{array}$ & 283 & 0.990 & 93.640 & 96.393 & 240 \\
\hline $\begin{array}{r}\text { Chionochloa } \\
\text { rubra }\end{array}$ & 97 & 0.989 & 91.751 & 97.461 & 239 \\
\hline $\begin{array}{l}\text { Coprosma } \\
\text { perpusilla }\end{array}$ & 61 & 0.995 & 98.361 & 96.281 & 239 \\
\hline $\begin{array}{l}\text { Dracophyllum } \\
\text { recurvum }\end{array}$ & 232 & 0.991 & 94.828 & 97.860 & 235 \\
\hline $\begin{array}{r}\text { Gentainella } \\
\text { bellidifolia }\end{array}$ & 165 & 0.986 & 92.727 & 99.103 & 240 \\
\hline
\end{tabular}

\section{Responses to heather}

The responses of individual taonga tipu to competition with $C$. vulgaris were investigated using percentage cover data collected within quadrats across TNP. We subset the total survey data $(n$ $=490$ ) to only include those survey locations where presence was predicted by climate envelope models for $C$. vulgaris and each taonga tipu. We excluded plots where $C$. vulgaris was absent to decrease the effect of dispersal limitation. The density of taonga tipu, as a function of the density of the invasive heather within each quadrat was analysed using boosted regression trees (BRT) in the package 'gbm' in R. A typical feature of ecological datasets is non-linear data, particularly when global environmental change is incorporated. A useful technique to explore these data is BRT, particularly as it is robust to permutations in the training data. BRTs were calibrated with a learning rate of 0.01 , a bag fraction of 0.5 and a step size of 0.1 . As the purpose of the analysis was to identify thresholds rather than closely model the data, tree 
complexity was set to 1 . BRT were generally poorly descriptive, therefore the viability of each partition identified by the BRT was further supported with Wilcoxon-signed rank tests using the function 'wilcox.test'. We expect that the poor descriptive power of our BRT is primarily a result of zero-inflated data, which is typical of ecological datasets.

\section{Environmental suitability-density relationship}

Next, we predicted the area of TNP susceptible to 'dense' invasion of $C$. vulgaris, where 'dense' was defined differently for each taonga tipu, depending on that species' response to $C$. vulgaris density as determined by BRT. Upper limits of $C$. vulgaris density were predicted using the $C$. vulgaris climate envelope model. To achieve this, we extracted environmental suitability scores from the $C$. vulgaris climate envelope model for all quadrat locations. We derived a series of relationships between $C$. vulgaris environmental suitability and percentage cover using quantile-regression in the 'quantreg' package v 5.36. We calculated regressions for the $50^{\text {th }}$, $60^{\text {th }}, 70^{\text {th }}, 80^{\text {th }}, 90^{\text {th }}$, and $97^{\text {th }}$ quantiles. We determined standard error for each quantile using 200 bootstraps and calculated Nagelkerke's $r^{2}$ to estimate predictive power, using the ' $r q^{\prime}$ function in the package 'rcompanion', v 1.13.2. Both least squares regression and all quantile regressions showed a positive relationship between predicted environmental suitability and $C$. vulgaris density (Supplementary Figure 16A). However, explanatory power was highest for upper quantiles: at the $90^{\text {th }}$ percentile, $65 \%$ of variance in C. vulgaris density was explained by predicted environmental suitability, significantly more than the $20 \%$ explained by ordinary least squares regression ( $t=10.84, p<0.001$; Supplementary Figure 16B). We therefore used the $90^{\text {th }}$ quantile regression to predict potential C. vulgaris densities across TNP. 


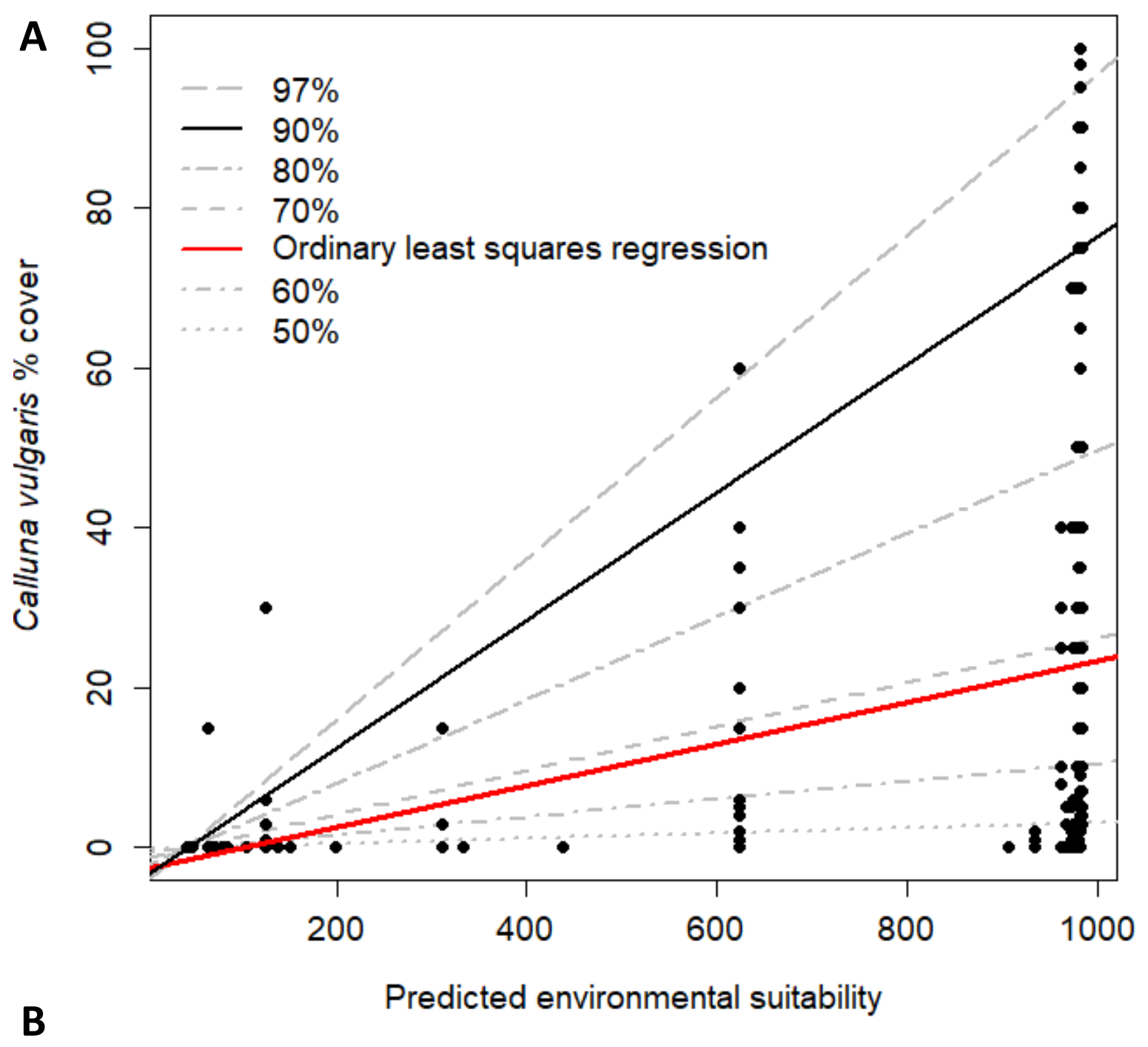

\begin{tabular}{|c|c|c|c|c|c|}
\hline Quantile & Estimate & Standard Error & t value & p value & Nagelkerke $r^{2}$ \\
\hline 0.50 & 0.003 & 0.002 & 2.181 & 0.030 & 0.020 \\
\hline 0.60 & 0.011 & 0.005 & 2.064 & 0.040 & 0.112 \\
\hline 0.70 & 0.027 & 0.005 & 5.764 & $<0.001$ & 0.330 \\
\hline 0.80 & 0.052 & 0.007 & 7.188 & $<0.001$ & 0.520 \\
\hline 0.90 & 0.080 & 0.007 & 11.390 & $<0.001$ & 0.653 \\
\hline 0.97 & 0.101 & 0.002 & 42.735 & $<0.001$ & 0.730 \\
\hline
\end{tabular}

Supplementary Figure 16: Performance of quantile regression in predictive Calluna vulgaris percentage cover as a function of environmental suitability: A) Percentage cover of $C$. vulgaris as a function of environmental suitability, as predicted by an ensemble climatic model. Data are presented together with relationship estimates by ordinary least squares regression (red) and 97th, 90th, 80th, 70th, $60^{\text {th }}$ and $50^{\text {th }}$ quantile regressions (grey and black); and B) performance of six quantile regressions in predicting percentage cover of $C$. vulgaris as a function of environmental suitability in Tongariro National Park. 
We transformed climate model projections of $C$. vulgaris distributions under the current and future climates into three partitions, based on two thresholds. The purpose of this first partition was to delineate areas in which $C$. vulgaris does and does not occur. The first partition was based on ROC curves, as described above. The purpose of the second partition was to delineate areas in which it is possible for $C$. vulgaris to reach high densities that are associated with depressed densities of each taonga tipu. To achieve this, we used the 'predict.rq' function in the 'quantreg' package to predict the environmental suitability value associated with the $C$. vulgaris density threshold identified by BRT. Finally, we overlaid taonga tipu spatial projections with spatial projections of $C$. vulgaris in each climate scenario. We calculated the proportion of TNP land area corresponding to each combination of predictions for the two species.

\section{Predictions}

\section{European Heather- Calluna vulgaris}

Calluna vulgaris was most responsive to

temperature variables, with mean summertime

temperatures being the most important predictor of its distribution. The species is environmentally excluded where the mean summertime temperature is below $12^{\circ} \mathrm{C}$. Temperature variability across the day also explained some of its distribution, with areas which change by more than $9.9^{\circ} \mathrm{C}$ across a day being

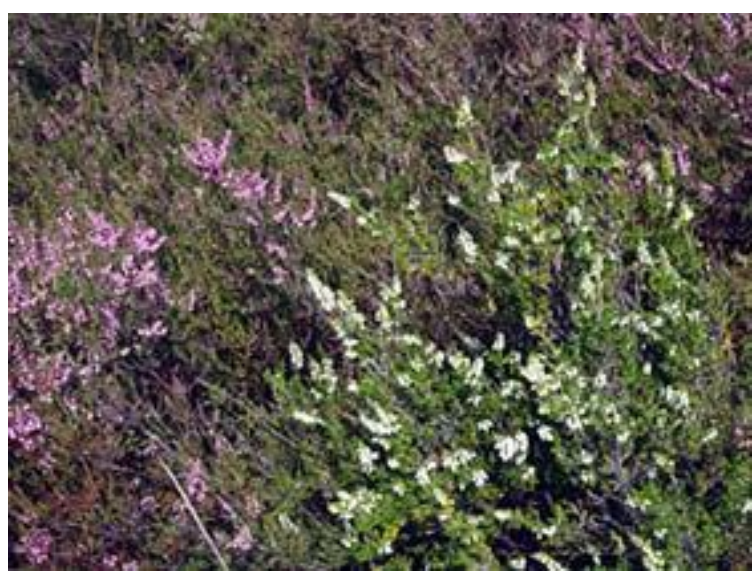
unsuitable (

\section{Supplementary Table 5).}

Supplementary Table 5: Environmental predictors used to calibrate the climatic model for Calluna vulgaris together with their relative importance and the role they play in limiting the distribution of this species.

\begin{tabular}{|l|l|l|l|l|}
\hline Variable & Code & Importance & Lower limit & Upper limit \\
\hline Mean Diurnal Temperature Range & Bio_2 & 0.13 & Unknown & $9.9^{\circ} \mathrm{C}$ \\
\hline Mean Temperature - Warmest Quarter & Bio_10 & 0.93 & $12^{\circ} \mathrm{C}$ & $20^{\circ} \mathrm{C}$ \\
\hline
\end{tabular}




\section{Distribution change}

The geographic area of TNP which is climatically suitable for $C$. vulgaris is indicated in Supplementary Figure 17. By the year 2070, we expect the climate to facilitate the movement of $C$. vulgaris to higher elevations in TNP. This trend holds true for both moderate and severe climate scenarios, however the elevational expansion predicted for $C$. vulgaris is larger under the more severe climate scenario. This prediction means that in the future, a larger area of TNP will be climatically suitable for the species.

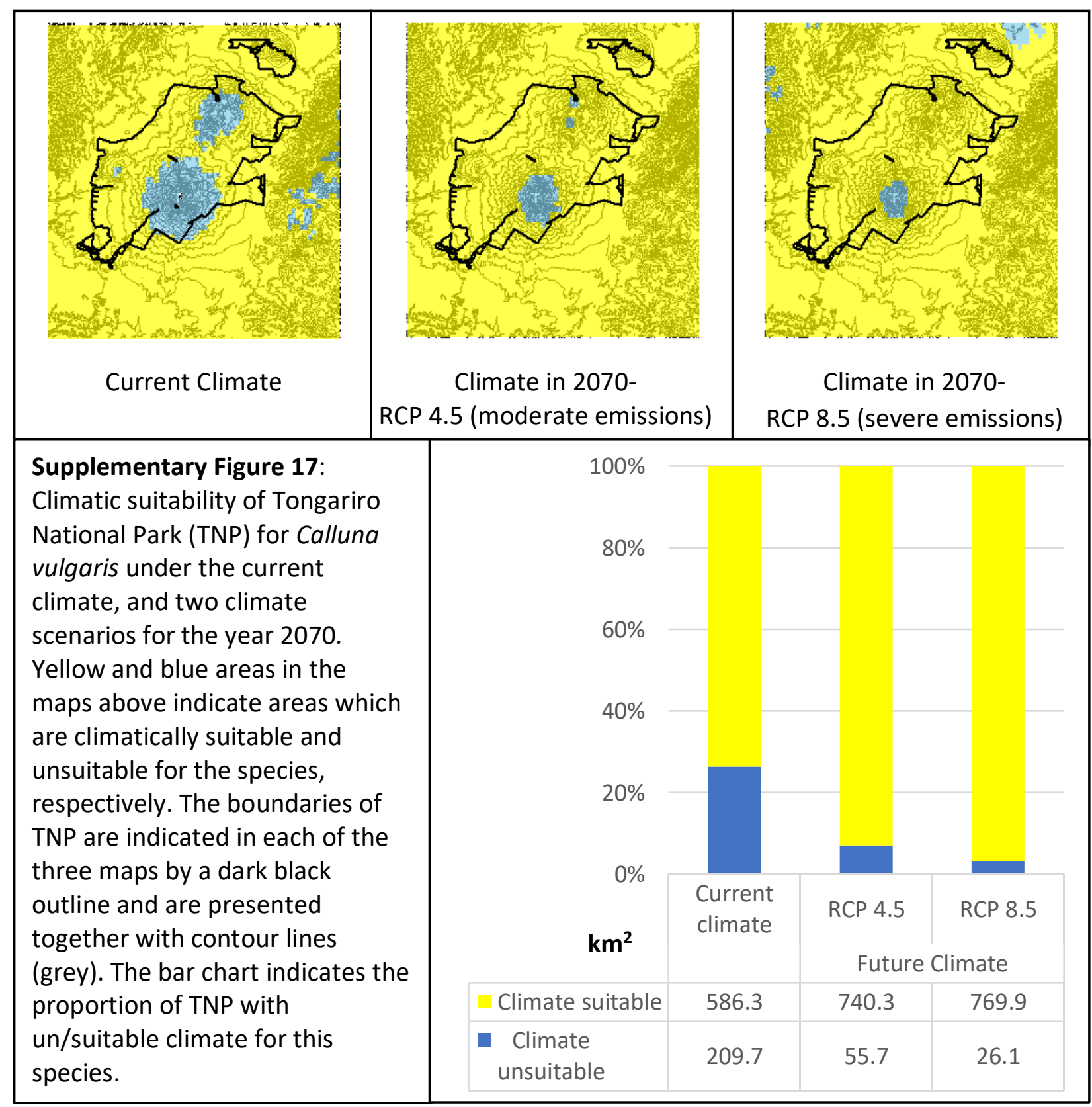




\section{Anisotome aromatica- Kopoti}

\section{Response to climate}

Anisotome aromatica was most responsive to temperature and water variables, with mean winter temperature and precipitation of the wettest week being the most important predictors of the species' distribution. The species is environmentally excluded where the mean winter temperature is more than $6.5^{\circ} \mathrm{C}$, or where precipitation of the wettest week is

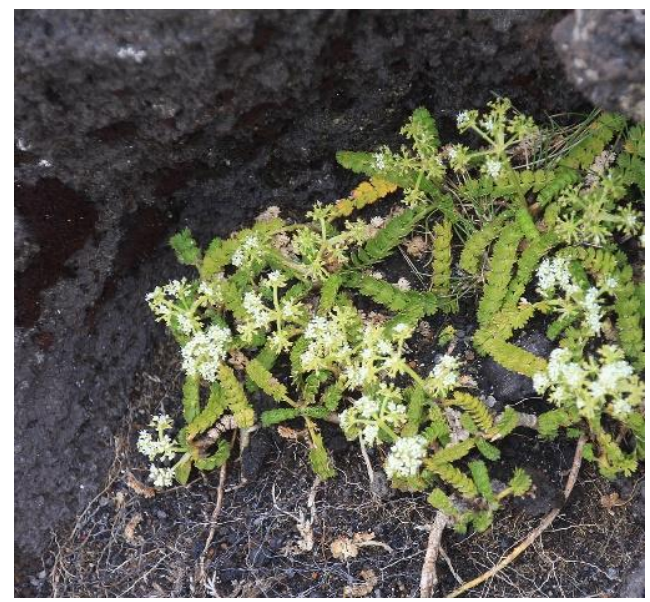
less than 200mm (Supplementary Table 6).

Supplementary Table 6: Environmental predictors used to calibrate the climatic model for Anisotome aromatica together with their relative importance and the role they play in limiting the distribution of this species.

\begin{tabular}{|l|l|l|l|l|}
\hline Variable & Code & Importance & Lower limit & Upper limit \\
\hline Mean Temperature - Coldest Quarter & Bio_11 & 0.89 & Unknown & $6.5^{\circ} \mathrm{C}$ \\
\hline Precipitation - Wettest Week & Bio_13 & 0.15 & $200 \mathrm{~mm}$ & Unknown \\
\hline
\end{tabular}

\section{Response to heather}

The density of $A$. aromatica is negatively associated with increasing $C$. vulgaris cover

(Supplementary Figure 18). Plots with a C. vulgaris density of more than $82.5 \%$ support significantly less $A$. aromatica than plots with no $C$. vulgaris. Plots above this threshold had an average $A$. aromatica cover of $0.06 \%$ cover, in comparison with plots where $C$. vulgaris is absent which had an average $A$. aromatica cover of $0.80 \%(W=2652, p<0.001)$. This 13 -fold decrease in average $A$. aromatica cover is a very strong response to high $C$. vulgaris densities. 


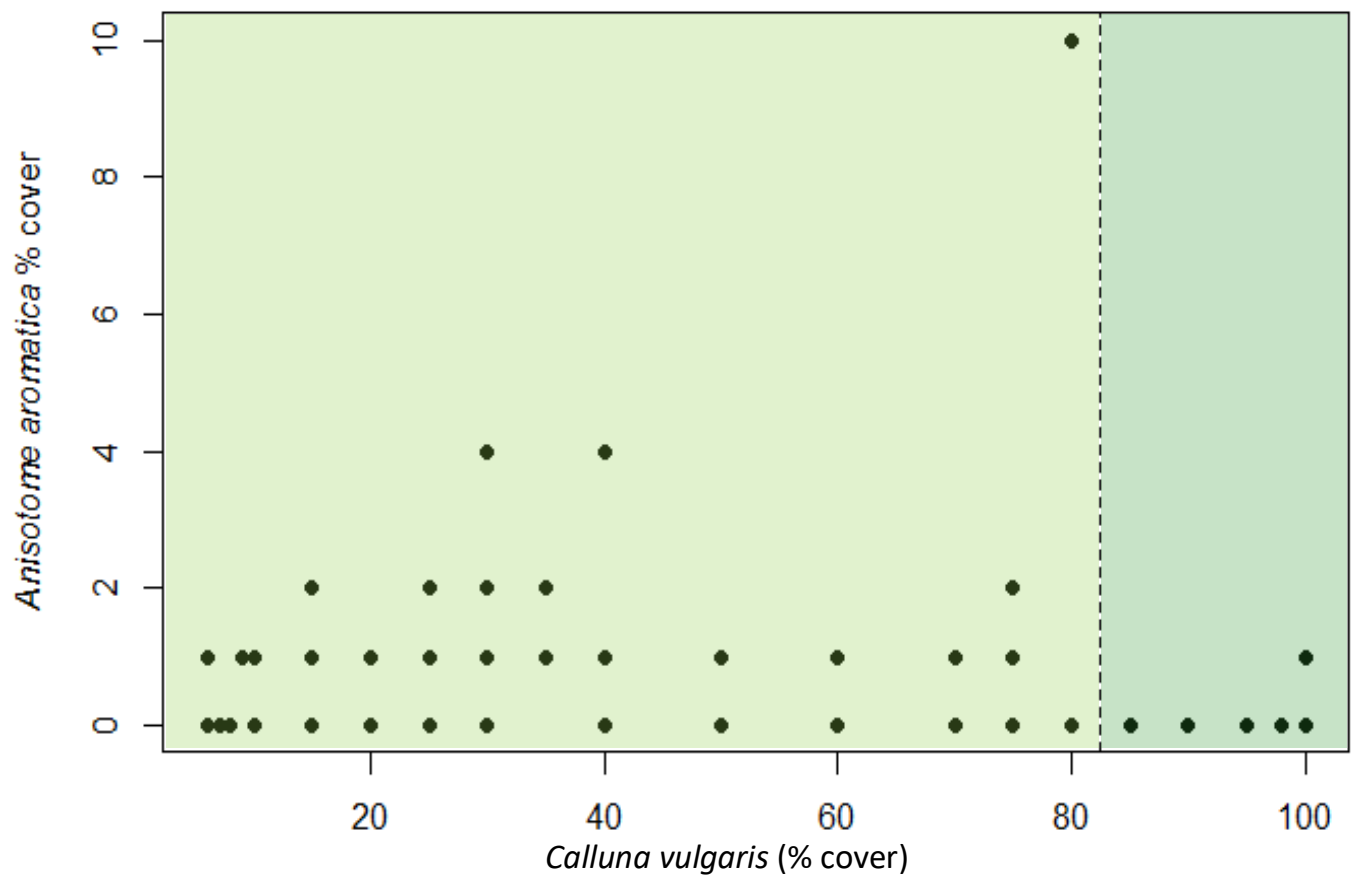

Supplementary Figure 18: The percentage cover of Anisotome aromatica decreases with increasing percentage cover of Calluna vulgaris across plots in Tongariro National Park. The data is presented together with the most significant threshold in the relationship (at $82.5 \% \mathrm{C}$. vulgaris cover), as identified by Boosted Regression Tree analysis.

\section{Distribution change}

We expect a large reduction in the distribution of $A$. aromatica under both future climate scenarios relative to its current day distribution (Supplementary Figure 19). This reduction is driven by both the influence of a changing climate acting directly on A. aromatica as well as the uphill movement of $C$. vulgaris in response to these climatic changes. Within the geographic space where we expect $A$. aromatica and $C$. vulgaris to co-occur, we expect that under all climatic conditions, the majority of space will be climatically suitable for $C$. vulgaris to form very high-density patches which are associated with large declines in A. aromatica populations. 


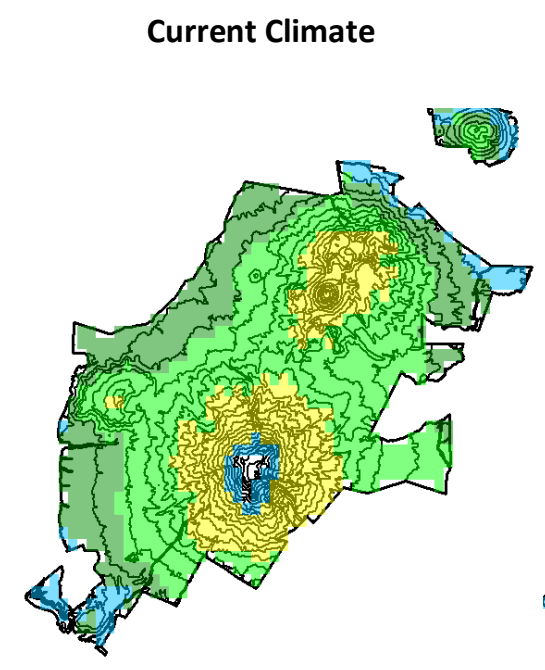

Current Climate

RCP 4.5 (moderate emissions)

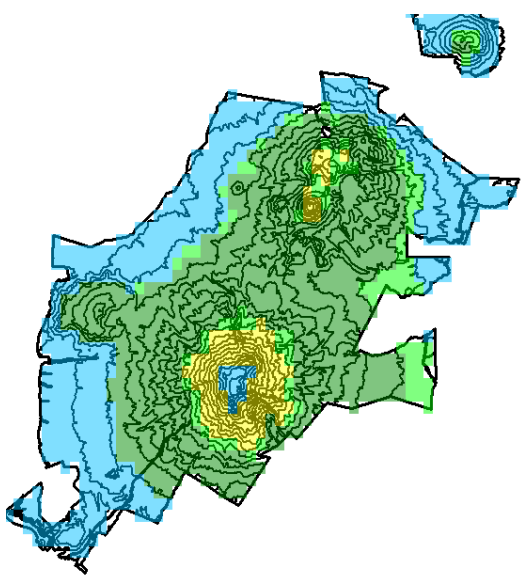

Climate in 2070-

RCP 8.5 (severe emissions)

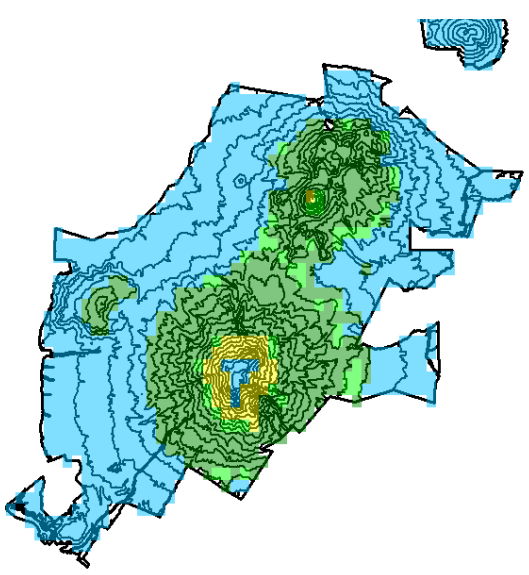

Supplementary Figure 19: Climatic suitability of Tongariro National Park (TNP) for Anisotome aromatica and the space within which it interacts with Calluna vulgaris under the current climate, and two climate scenarios for the year 2070. Blue indicates areas which are climatically unsuitable for $A$. aromatica. Green areas indicate spaces within which $A$. aromatica may co-occur with C. vulgaris. Dark green indicates areas where the climate is sufficiently suitable for $C$. vulgaris to reach densities of more than $82.5 \%$ and may lead to serious decreases in the density of $A$. aromatica; light green areas indicate marginal habitats for $C$. vulgaris which will not significantly impact $A$. aromatica. Yellow areas indicate spaces in which the climate is suitable for $A$. aromatica but not for $C$. vulgaris. The boundaries of TNP are indicated in each of the three maps by a dark black outline and are presented together with contour lines (grey). The bar chart quantifies the space within each category

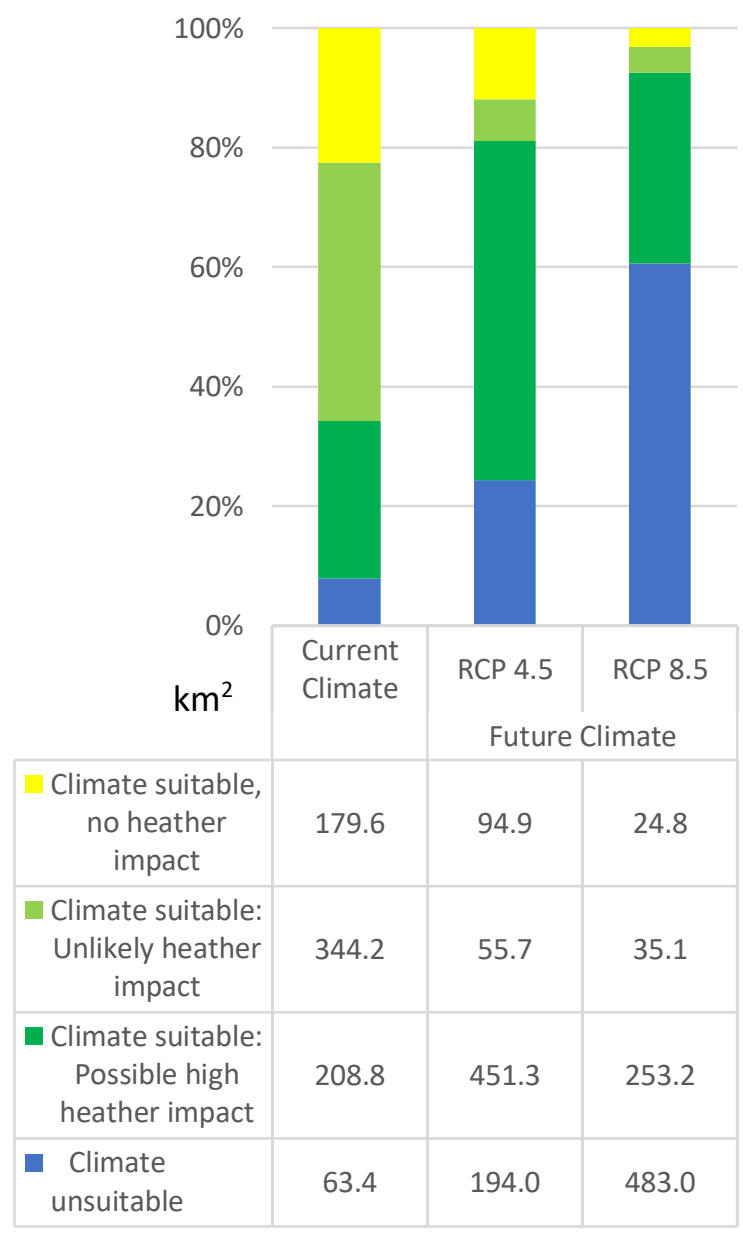
$\left(\mathrm{km}^{2}\right)$. 


\section{Chionochloa rubra- Red Tussock}

\section{Response to climate}

Chionochloa rubra was most responsive to temperature and water variables, with mean temperature and total precipitation of the wettest quarter of the year being the most important predictors of the species' distribution. The species is environmentally excluded where the mean temperature of the wettest season is more than $6.5^{\circ} \mathrm{C}$, or where precipitation is less than 600mm (Supplementary Table 7).

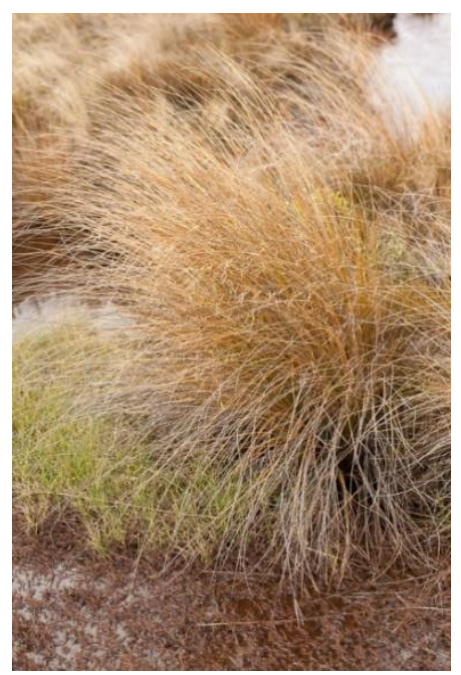

Supplementary Table 7: Environmental predictors used to calibrate the climatic model for Chionochloa rubra together with their relative importance and the role they play in limiting the distribution of this species.

\begin{tabular}{|l|l|l|l|l|}
\hline Variable & Code & Importance & Lower limit & Upper limit \\
\hline Mean Temperature - Wettest Quarter & Bio_8 & 0.87 & Unknown & $6.5^{\circ} \mathrm{C}$ \\
\hline Precipitation - Wettest Quarter & Bio_16 & 0.15 & $600 \mathrm{~mm}$ & Unknown \\
\hline
\end{tabular}

\section{Response to heather}

The density of $C$. rubra is negatively associated with increasing $C$. vulgaris cover (

Supplementary Figure 20). Plots with a $C$. vulgaris density of more than $77.5 \%$ support significantly less $C$. rubra than plots with no $C$. vulgaris. Plots above this $C$. vulgaris threshold had an average $C$. rubra cover of $0.84 \%$ cover, in comparison with plots where $C$. vulgaris is absent which had an average $C$. rubra cover of $6.04(W=1087, p=0.01)$. This 8 -fold decrease in average $C$. rubra cover is a strong response to high $C$. vulgaris densities. This supports previous observational data which indicates that the expansion of dense $C$. vulgaris leads to drastic declines in C. rubra density within TNP. 


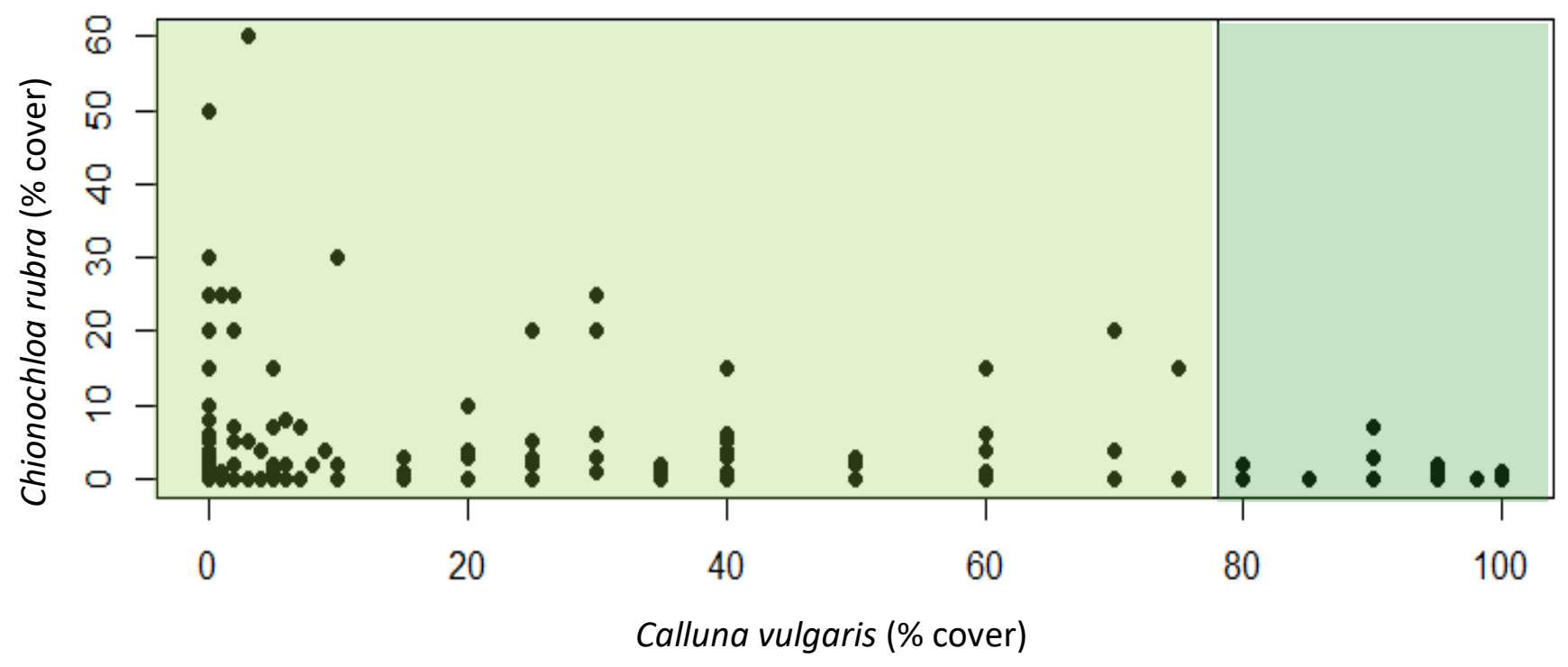

Supplementary Figure 20: The percentage cover of Chionochloa rubra decreases with increasing percentage cover of Calluna vulgaris across plots in Tongariro National Park. The data is presented together with the most significant threshold in the relationship (at $77 \% C$. vulgaris cover), as identified by Boosted Regression Tree analysis.

\section{Distribution change}

We expect a large reduction in the distribution of $C$. rubra under both future climate scenarios relative to its current day distribution (Supplementary Figure 21). This reduction is driven by both the influence of a changing climate acting directly on C. rubra as well as the uphill movement of $C$. vulgaris in response to these climatic changes. Within the geographic space where we expect $C$. rubra and $C$. vulgaris to co-occur, we expect that under all climatic conditions, the vast majority of space will be climatically suitable for $C$. vulgaris to form very high-density patches which are associated with large declines in C. rubra populations. 


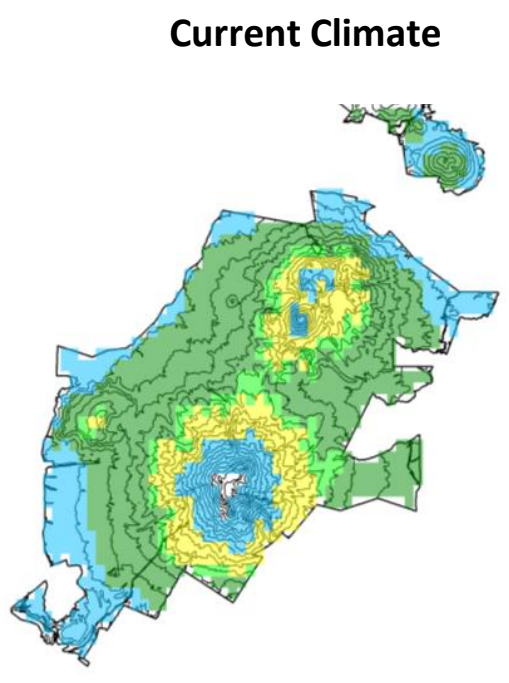

\section{Current Climate}

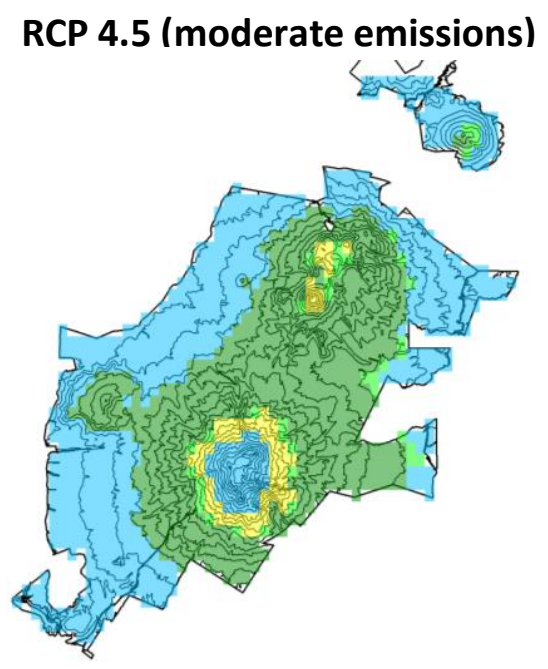

Climate in 2070-

RCP 8.5 (severe emissions)

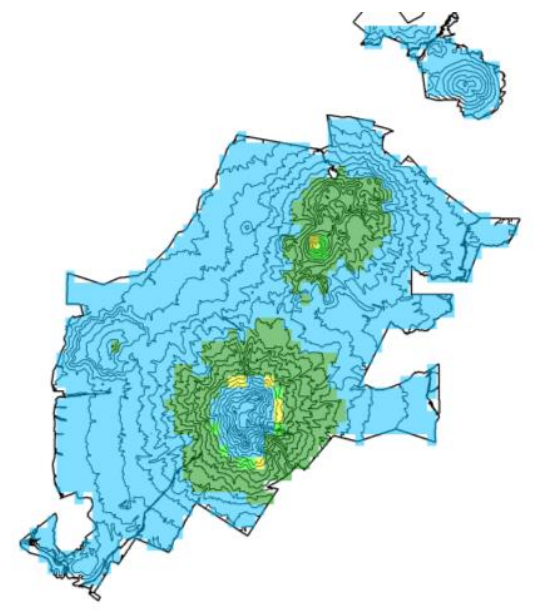

Supplementary Figure 21: Climatic suitability of Tongariro National Park (TNP) for Chionochloa rubra and the space within which it interacts with Calluna vulgaris under the current climate, and two climate scenarios for the year 2070. Blue indicates areas which are climatically unsuitable for $C$. rubra. Green areas indicate spaces within which $C$. rubra may co-occur with $C$. vulgaris. Dark green indicates areas where the climate is sufficiently suitable for $C$. vulgaris to reach densities of more than $77 \%$ and may lead to significant decreases in the density of $C$. rubra; light green areas indicate marginal habitats for $C$. vulgaris where it will not significantly impact $C$. rubra. Yellow areas indicate spaces in which the climate is suitable for $C$. rubra but not for $C$. vulgaris. The boundaries of TNP are indicated in each of the three maps by a dark black outline and are presented together with contour lines (grey). The bar chart quantifies the space within each category $\left(\mathrm{km}^{2}\right)$.

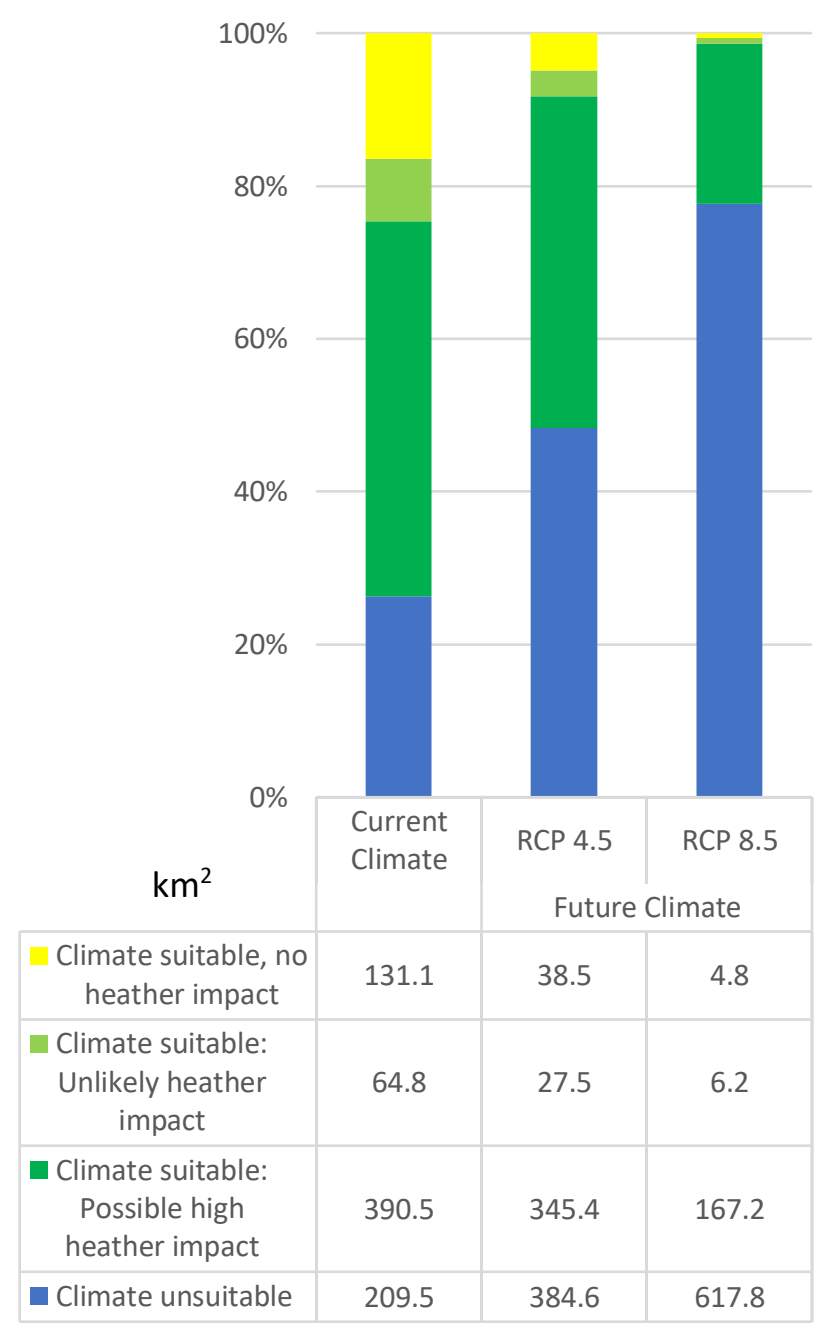




\section{Coprosma perpusilla- Creeping coprosma}

\section{Response to climate}

Coprosma perpusilla was most responsive to temperature and water variables, with mean daily temperature range and annual precipitation being the most important predictors of the species' distribution. The species is environmentally excluded where the mean daily temperature range is more than $9.5^{\circ} \mathrm{C}$, or where annual precipitation is less than

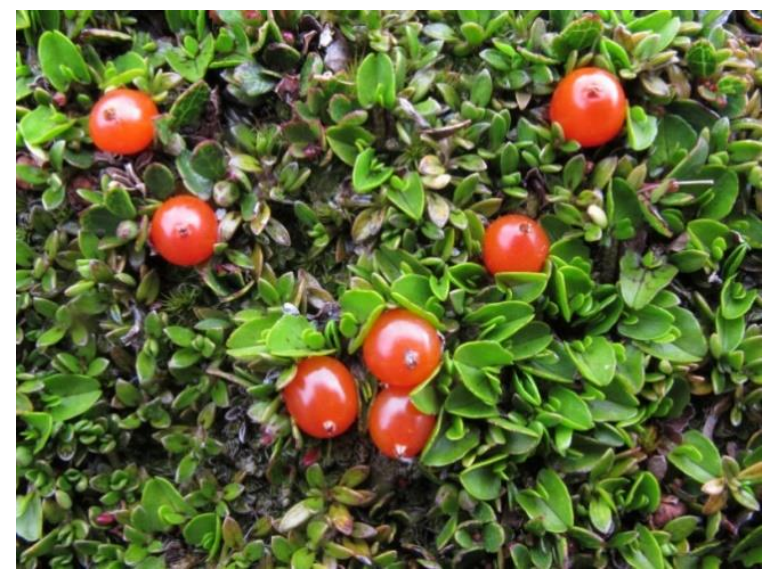
2000mm (Supplementary Table 8).

Supplementary Table 8: Environmental predictors used to calibrate the climatic model for Coprosma perpusilla together with their relative importance and the role they play in limiting the distribution of this species.

\begin{tabular}{|l|l|l|l|l|}
\hline Variable & Code & Importance & Lower limit & Upper limit \\
\hline Mean Diurnal Temperature Range & Bio_2 & 0.29 & Unknown & $9.5^{\circ} \mathrm{C}$ \\
\hline Annual Precipitation & Bio_12 & 0.82 & $2000 \mathrm{~mm}$ & Unknown \\
\hline
\end{tabular}

\section{Response to heather}

The density of $C$. perpusilla is negatively associated with increasing $C$. vulgaris cover (Supplementary Figure 22). Plots with a C. vulgaris density of more than $65 \%$ support significantly less $C$. perpusilla than plots with no $C$. vulgaris. Plots above this threshold had an average $C$. perpusilla cover of $0.38 \%$ cover, in comparison with plots where $C$. vulgaris is absent which had an average $C$. perpusilla cover of $0.93 \%(W=2652, p<0.001)$. This 2 -fold decrease in average $C$. perpusilla cover is a response to medium and high $C$. vulgaris densities. 


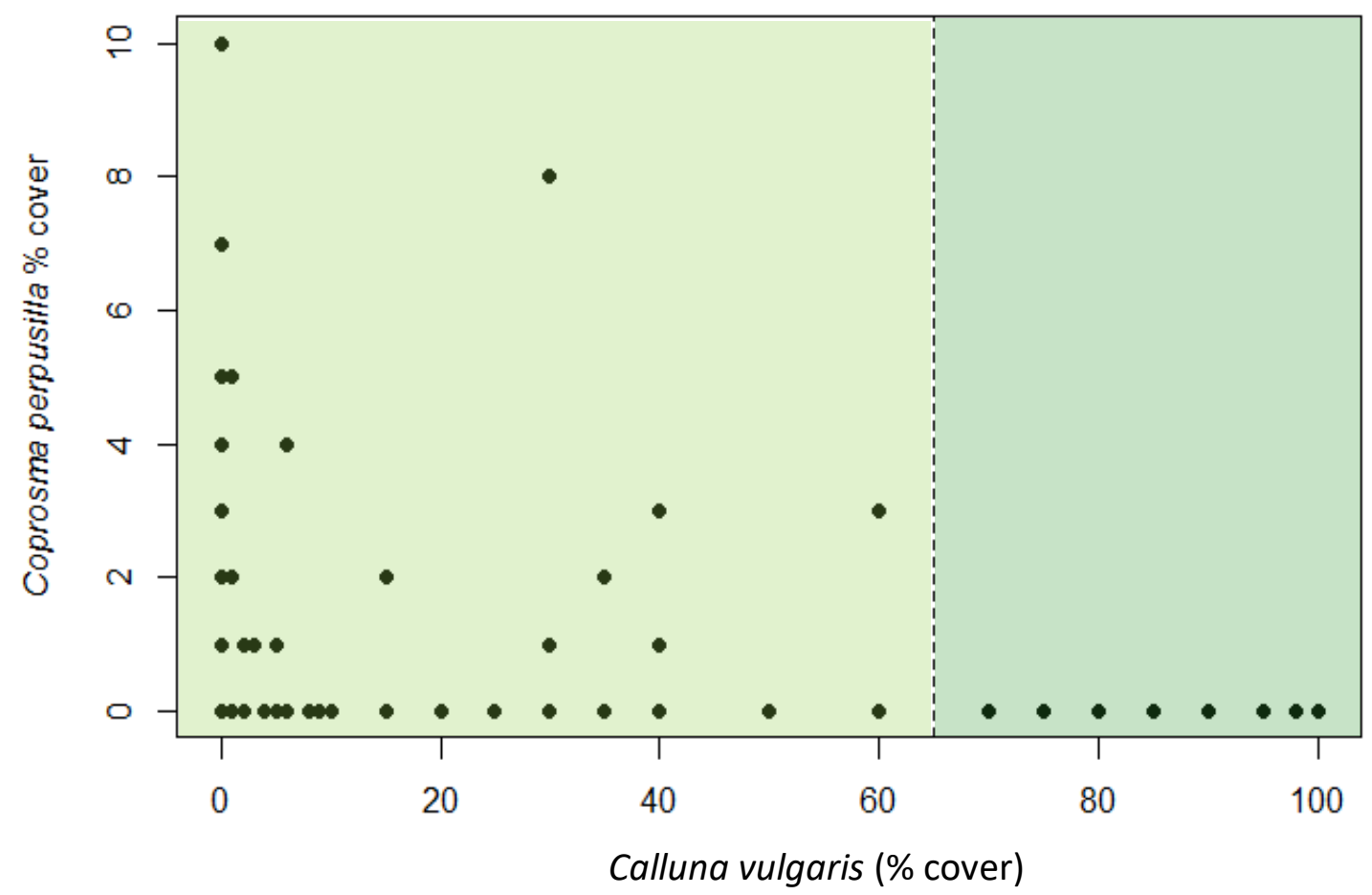

Supplementary Figure 22: The percentage cover of Coprosma perpusilla decreases with increasing percentage cover of Calluna vulgaris across plots in Tongariro National Park. The data is presented together with the most significant threshold in the relationship (at $77 \% C$. vulgaris cover), as identified by Boosted Regression Tree analysis.

\section{Distribution change}

We expect a large reduction in the distribution of $C$. perpusilla under both future climate scenarios relative to its current day distribution (Supplementary Figure 23). This reduction is driven primarily by the uphill movement of $C$. vulgaris in response to these climatic changes. Within the geographic space where we expect $C$. perpusilla and $C$. vulgaris to co-occur, we expect that under all climatic conditions, the vast majority of space will be climatically suitable for $C$. vulgaris to form very high-density patches which are associated with large declines in $C$. perpusilla populations. 

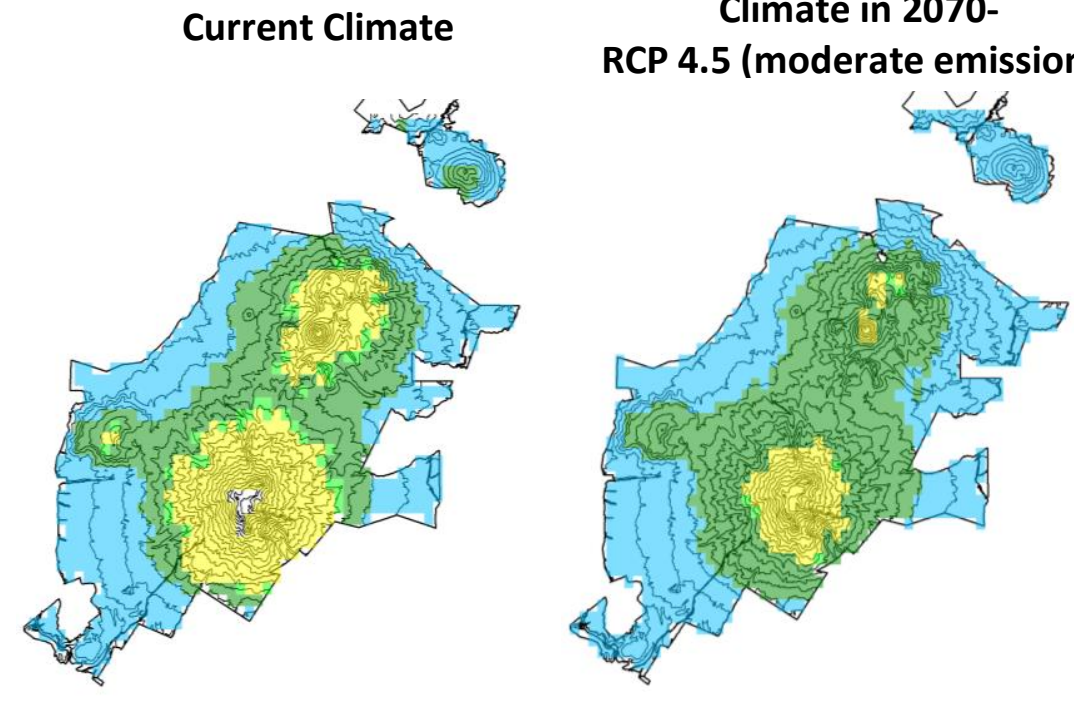

Climate in 2070-

RCP 8.5 (severe emissions)

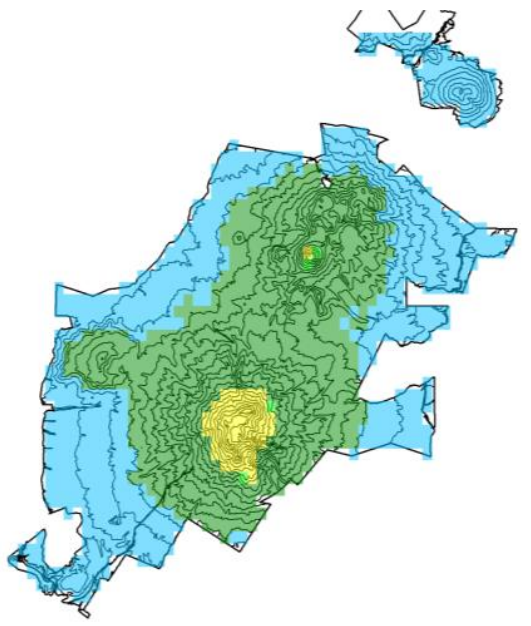

Supplementary Figure 23: Climatic suitability of Tongariro National Park (TNP) for Coprosma perpusilla and the space within which it interacts with Calluna vulgaris under the current climate, and two climate scenarios for the year 2070. Blue indicates areas which are climatically unsuitable for $C$. perpusilla. Green areas indicate spaces within which $C$. perpusilla may co-occur with $C$. vulgaris. Dark green indicates areas where the climate is sufficiently suitable for $C$. vulgaris to reach densities of more than $77 \%$ and may lead to serious decreases in the density of $C$. perpusilla; light green areas indicate marginal habitats for $C$. vulgaris will it will not significantly impact $C$. perpusilla. Yellow areas indicate spaces in which the climate is suitable for $C$. perpusilla but not for C. vulgaris. The boundaries of TNP are indicated in each of the three maps by a dark black outline and are presented together with contour lines (grey). The bar chart quantifies the space within each category $\left(\mathrm{km}^{2}\right)$.

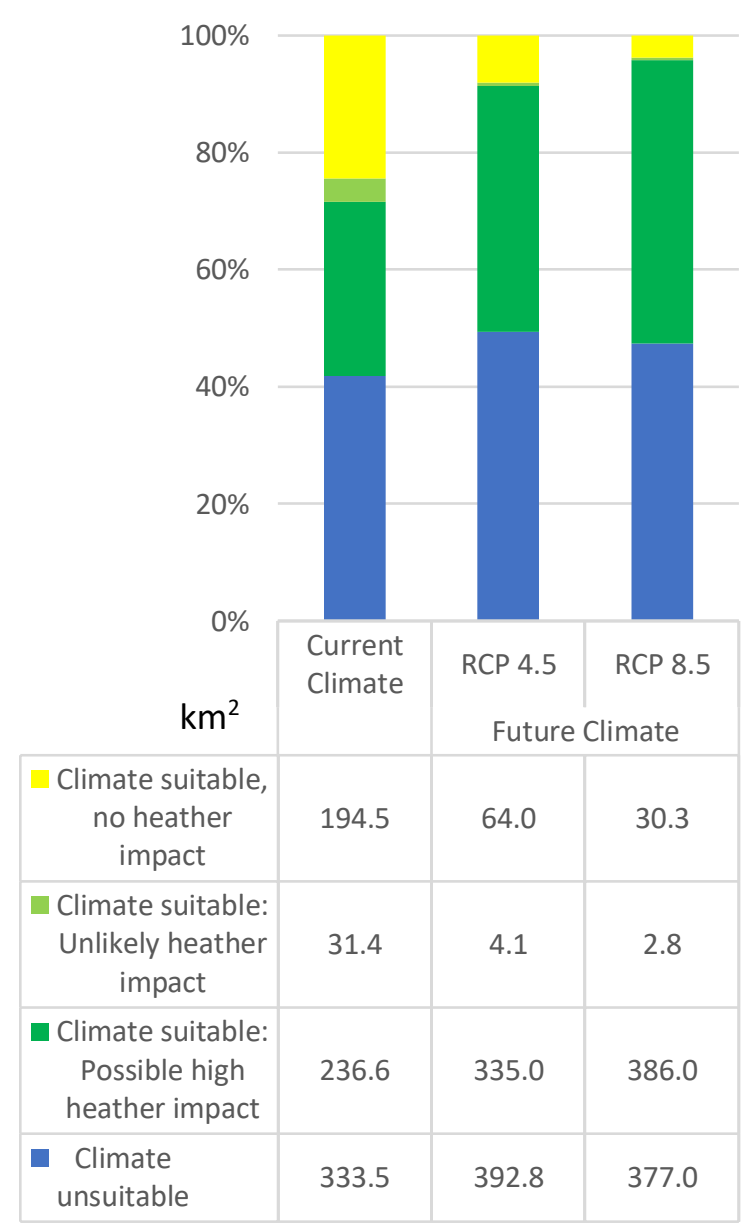




\section{Dracophyllum recurvum- Curly Leaved Nei-Nei}

\section{Response to climate}

Dracophyllum recurvum was most responsive to temperature and temperature variability, with temperature seasonality and mean temperature of the coldest quarter being the most important predictors of the species' distribution. The species is environmentally excluded where the temperature seasonality is less than $36^{\circ} \mathrm{C}$, or where mean temperature of the coldest quarter is more than $5^{\circ} \mathrm{C}$ (Supplementary Table 9).

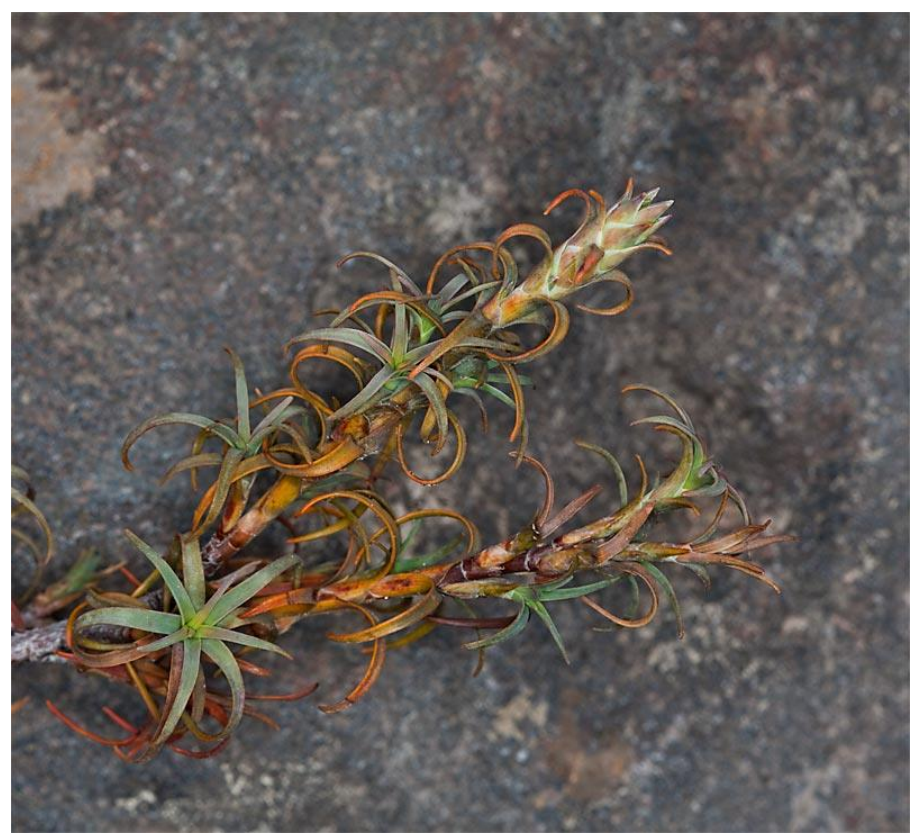

Supplementary Table 9: Environmental predictors used to calibrate the climatic model for Dracophyllum recurvum together with their relative importance and the role they play in limiting the distribution of this species.

\begin{tabular}{|l|l|l|l|l|}
\hline Variable & Code & Importance & Lower limit & Upper limit \\
\hline Temperature Seasonality & Bio_4 & 0.46 & $36{ }^{\circ} \mathrm{C}$ & Unknown \\
\hline Mean Temperature-Coldest Quarter & Bio_11 & 0.57 & Unknown & $5{ }^{\circ} \mathrm{C}$ \\
\hline
\end{tabular}

\section{Response to heather}

The density of $D$. recurvum is does not significantly change with increasing $C$. vulgaris cover (Supplementary Figure 24). Two thresholds (10 and $22.5 \%$ ) were identified by Boosted Regression Tree, however, neither was statistically supported. 


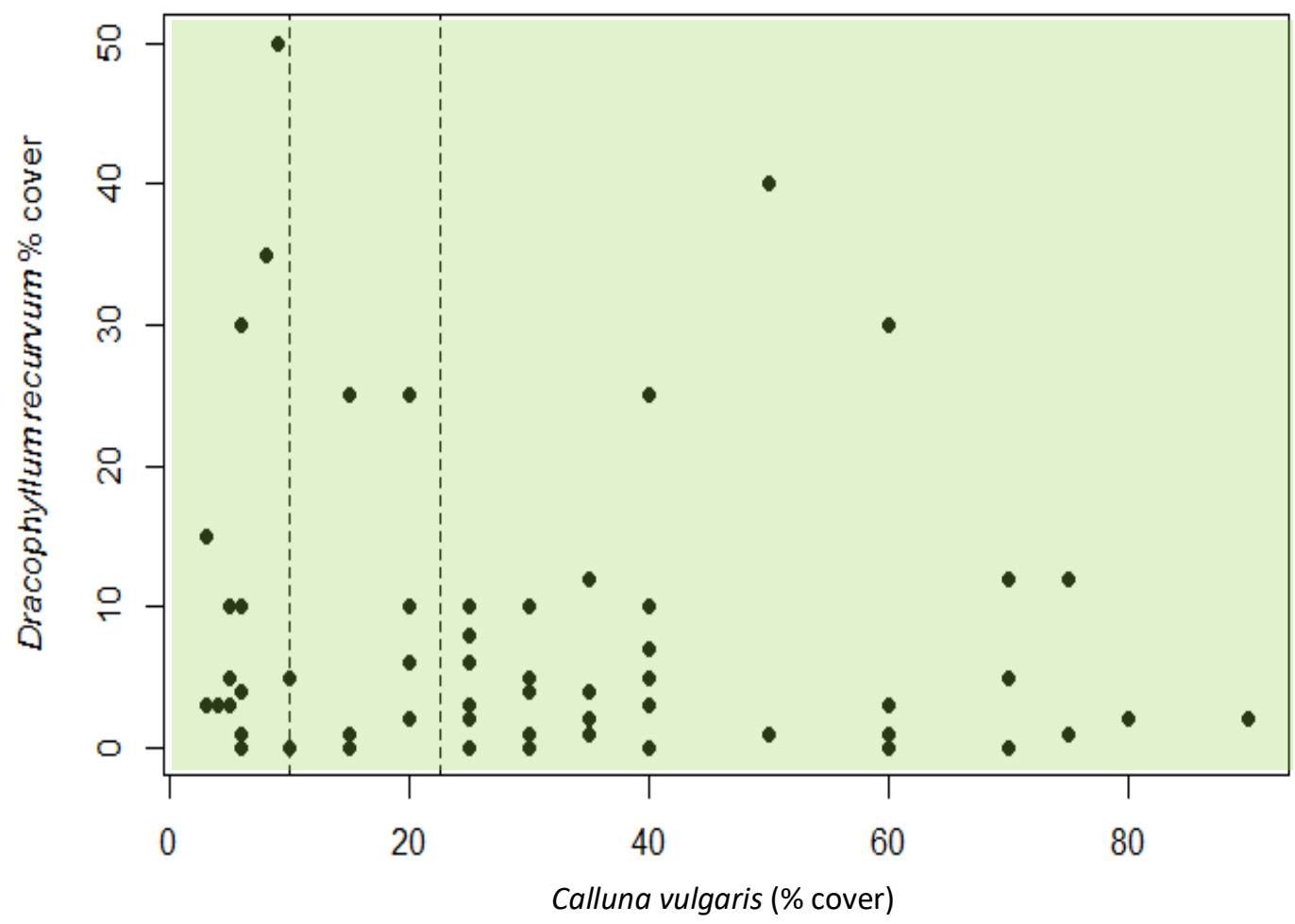

Supplementary Figure 24: The percentage cover of Dracophyllum recurvum is unaffected by the percentage cover of Calluna vulgaris across plots in Tongariro National Park. The data is presented together with the most significant thresholds in the relationship (at 10 and $22.5 \% C$. vulgaris cover), as identified by Boosted Regression Tree analysis however these were not significant.

\section{Distribution change}

We expect a large reduction in the distribution of $D$. recurvum under both future climate scenarios relative to its current day distribution (Supplementary Figure 25). This reduction is driven primarily by the influence of a changing climate acting directly on $D$. recurvum. We expect $D$. recurvum and $C$. vulgaris to co-occur under all climate scenarios, however we do not record any competitive impact of this co-occurrence on the density of $D$. recurvum. 


\section{Current Climate}

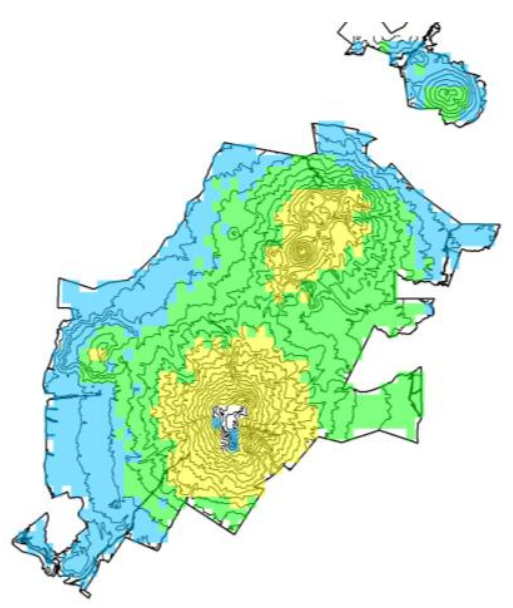

Climate in 2070-

RCP 4.5 (moderate emissions)

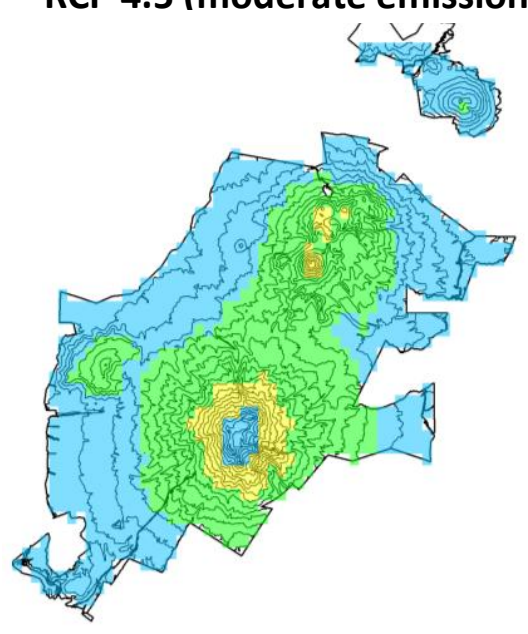

Climate in 2070-

RCP 8.5 (severe emissions)

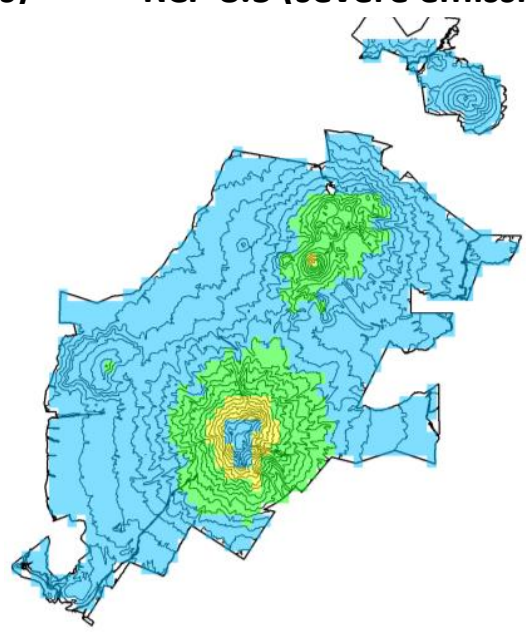

Supplementary Figure 25: Climatic suitability of Tongariro National Park (TNP) for Dracophyllum recurvum and the space within which it interacts with Calluna vulgaris under the current climate, and two climate scenarios for the year 2070. Blue indicates areas which are climatically unsuitable for $D$. recurvum. Green areas indicate spaces within which $D$. recurvum may co-occur with $C$. vulgaris, although this co-occurrence is unlikely to significantly impact $D$. subulatum. Yellow areas indicate spaces in which the climate is suitable for $D$. recurvum but not for $C$. vulgaris. The boundaries of TNP are indicated in each of the three maps by a dark black outline and are presented together with contour lines (grey). The bar chart quantifies the space within each category $\left(\mathrm{km}^{2}\right)$.

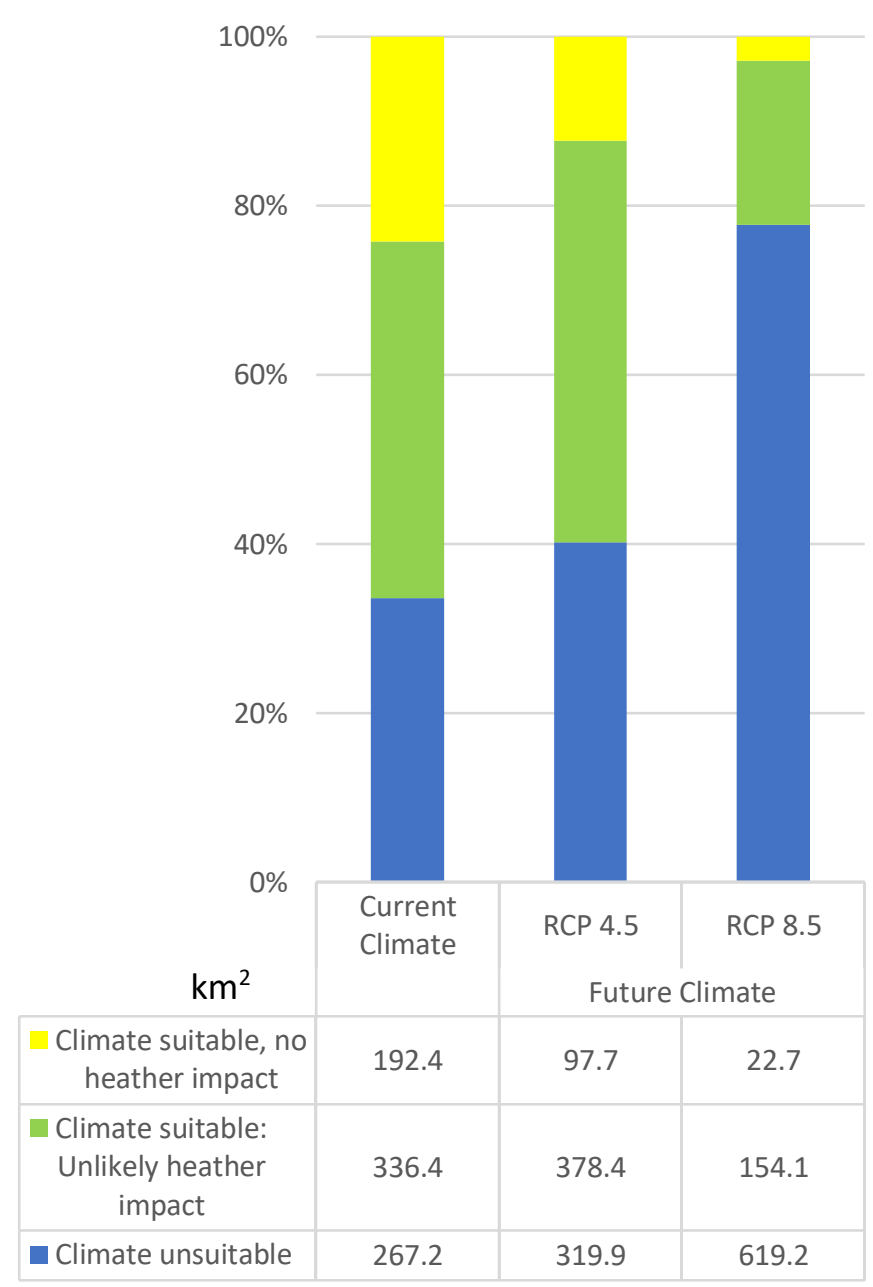




\section{Gentianella bellidifolia- Gentian}

\section{Response to climate}

The Gentianella bellidifolia was most responsive to temperature and temperature variability, with annual mean temperature and mean diurnal temperature range being the most important predictors of the species' distribution. The species is environmentally excluded where the annual mean temperature is more than $10^{\circ} \mathrm{C}$, or where

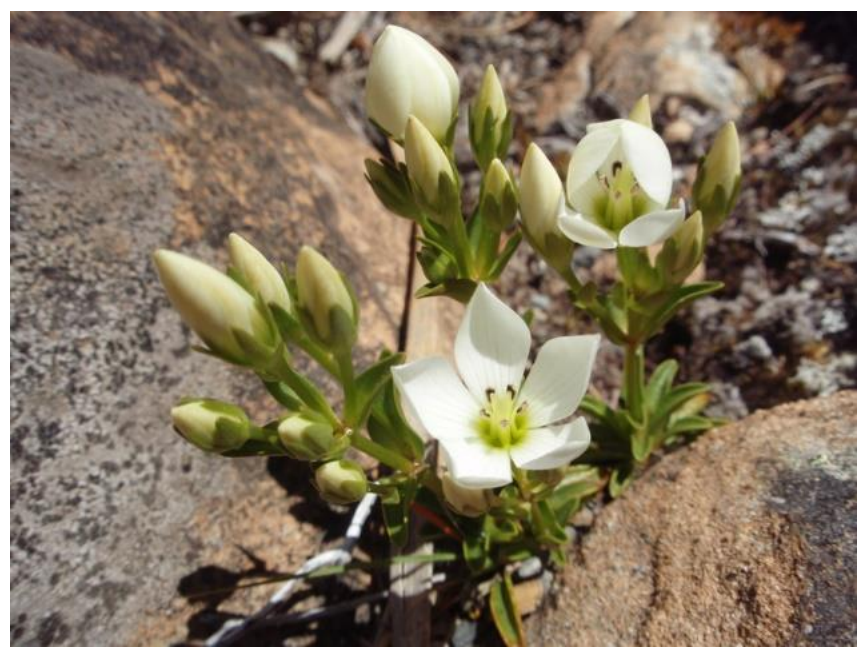
mean diurnal temperature range is more than $9.5^{\circ} \mathrm{C}$ or less than $8.5^{\circ} \mathrm{C}$ (Supplementary Table 10).

Supplementary Table 10: Environmental predictors used to calibrate the climatic model for Gentainella bellidifolia together with their relative importance and the role they play in limiting the distribution of this species.

\begin{tabular}{|l|l|l|l|l|}
\hline Variable & Code & Importance & Lower limit & Upper limit \\
\hline Annual Mean Temperature & Bio_1 & 0.67 & Unknown & $10^{\circ} \mathrm{C}$ \\
\hline Mean Diurnal Temperature Range & Bio_2 & 0.41 & $8.5^{\circ} \mathrm{C}$ & $9.5^{\circ} \mathrm{C}$ \\
\hline
\end{tabular}

\section{Response to heather}

The density of $G$. bellidifolia is does not significantly change with increasing $C$. vulgaris cover (Supplementary Figure 26). A threshold (10\%) was identified by Boosted Regression Tree, however it was not statistically supported. 


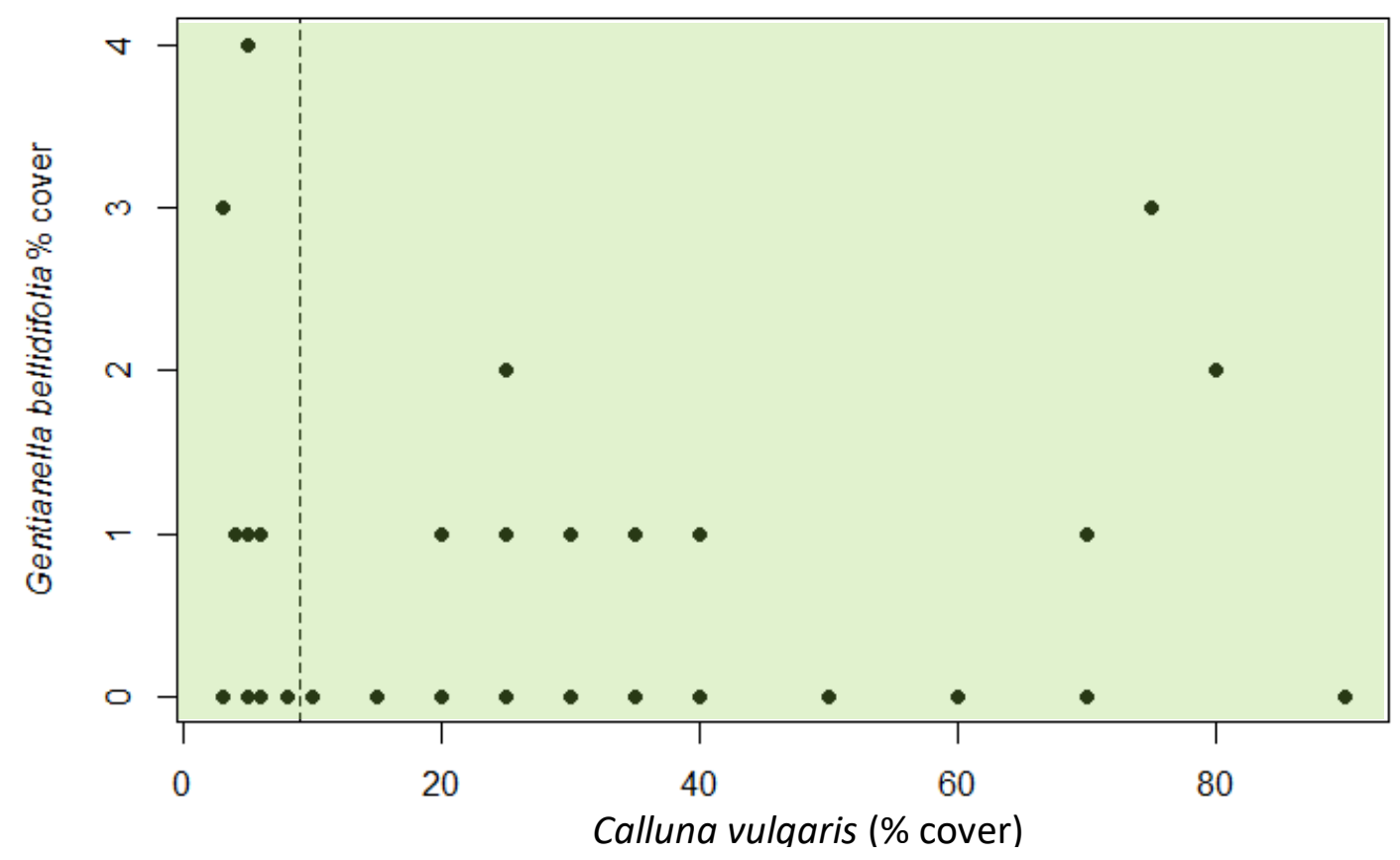

Supplementary Figure 26: The percentage cover of Gentianella bellidifolia is unaffected by the percentage cover of Calluna vulgaris across plots in Tongariro National Park. The data is presented together with the most significant threshold in the relationship (at $10 \%$. vulgaris cover), as identified by Boosted Regression Tree analysis however it was not significant .

\section{Distribution change}

We expect a large reduction in the distribution of $G$. bellidifolia under both future climate scenarios relative to its current day distribution (Supplementary Figure 27). This reduction is driven primarily by the influence of a changing climate acting directly on G. bellidifolia. We expect G. bellidifolia and C. vulgaris to co-occur under all climate scenarios, however we do not record any competitive impact of this co-occurrence on the density of G. bellidifolia. 


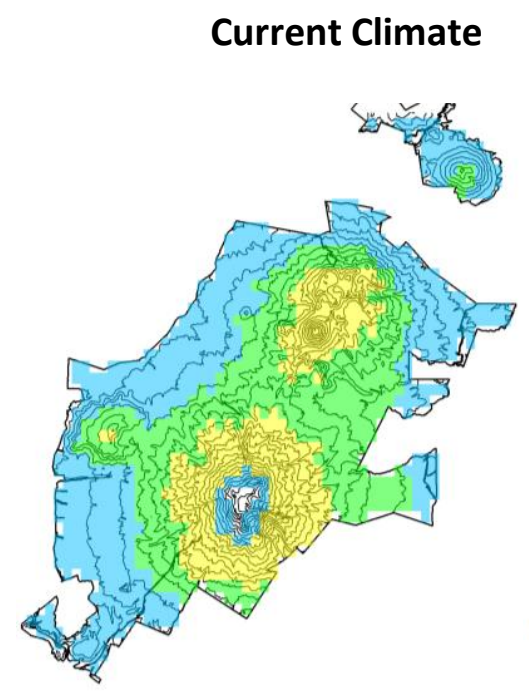

Supplementary Figure 27: Climatic suitability of Tongariro National Park (TNP) for Gentianella bellidifolia and the space within which it interacts with Calluna vulgaris under the current climate, and two climate scenarios for the year 2070. Blue indicates areas which are climatically unsuitable for $G$. bellidifolia. Green areas indicate spaces within which for $G$. bellidifolia may cooccur with $C$. vulgaris, although this cooccurrence is unlikely to significantly impact G. bellidifolia. Yellow areas indicate spaces in which the climate is suitable for $G$. bellidifolia but not for $C$. vulgaris. The boundaries of TNP are indicated in each of the three maps by a dark black outline and are presented together with contour lines (grey). The bar chart quantifies the space within each category $\left(\mathrm{km}^{2}\right)$.
Climate in 2070RCP 4.5 (moderate emissions) RCP 8.5 (severe emissions)
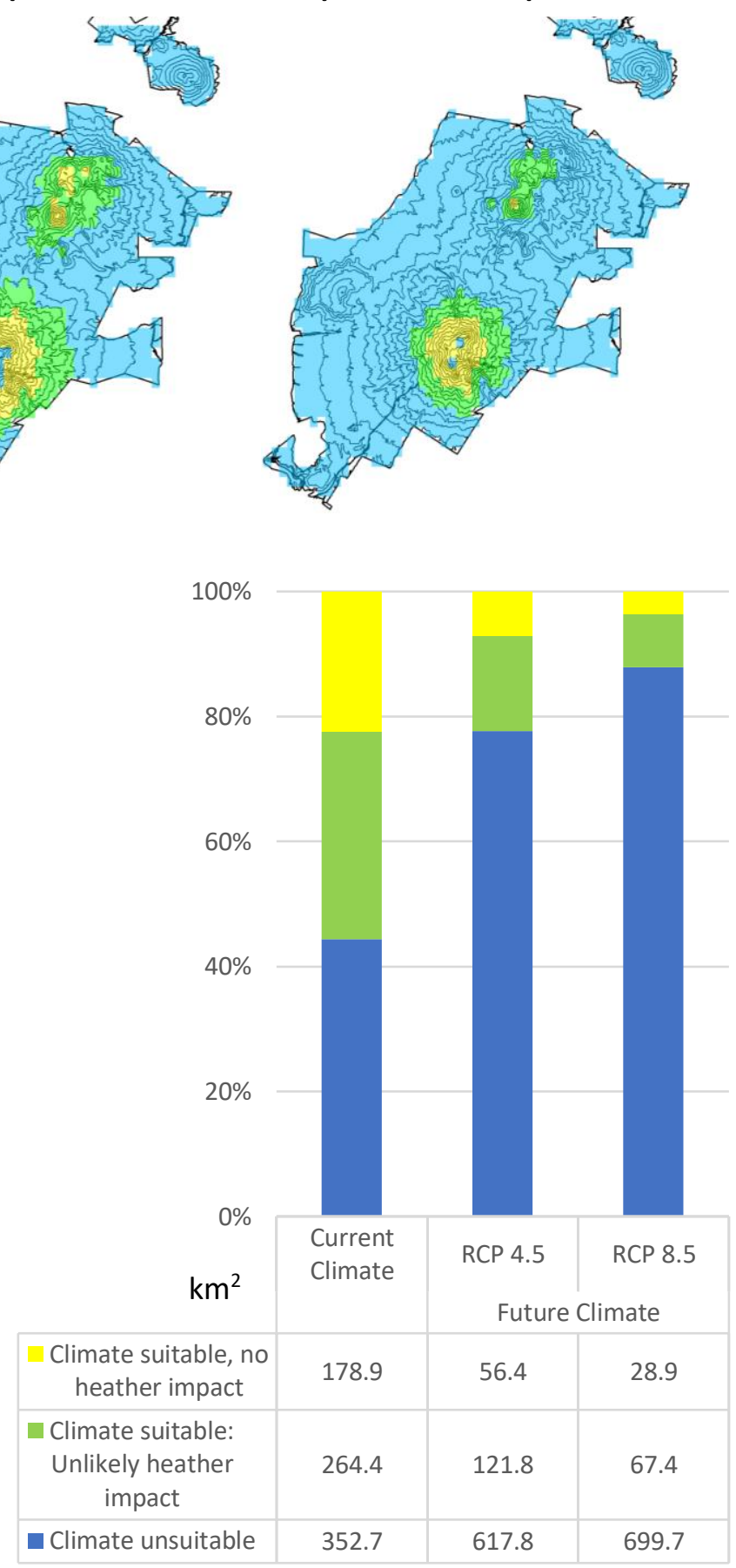


\section{Available habitat for Taonga Tipu in the future}

The taonga tipu responded idiosyncratically to the relative importance of $C$. vulgaris invasion and climate change. For example, the direct effects of climate change were important in reducing the habitat available for the high-alpine herb Gentianella bellidifolia and Dracophyllum recurvum, while increases in the distribution and abundance of $C$. vulgaris were more important in driving the declines of the three other species modelled. Despite this, our models predict universally smaller areas of suitable habitat within TNP, for all five species.

Supplementary Figure $\mathbf{2 8}$ summarises the combined effects of climate change and predicted changes in C. vulgaris distributions and densities on the area of suitable habitat in TNP in 2070, for the 5 species.

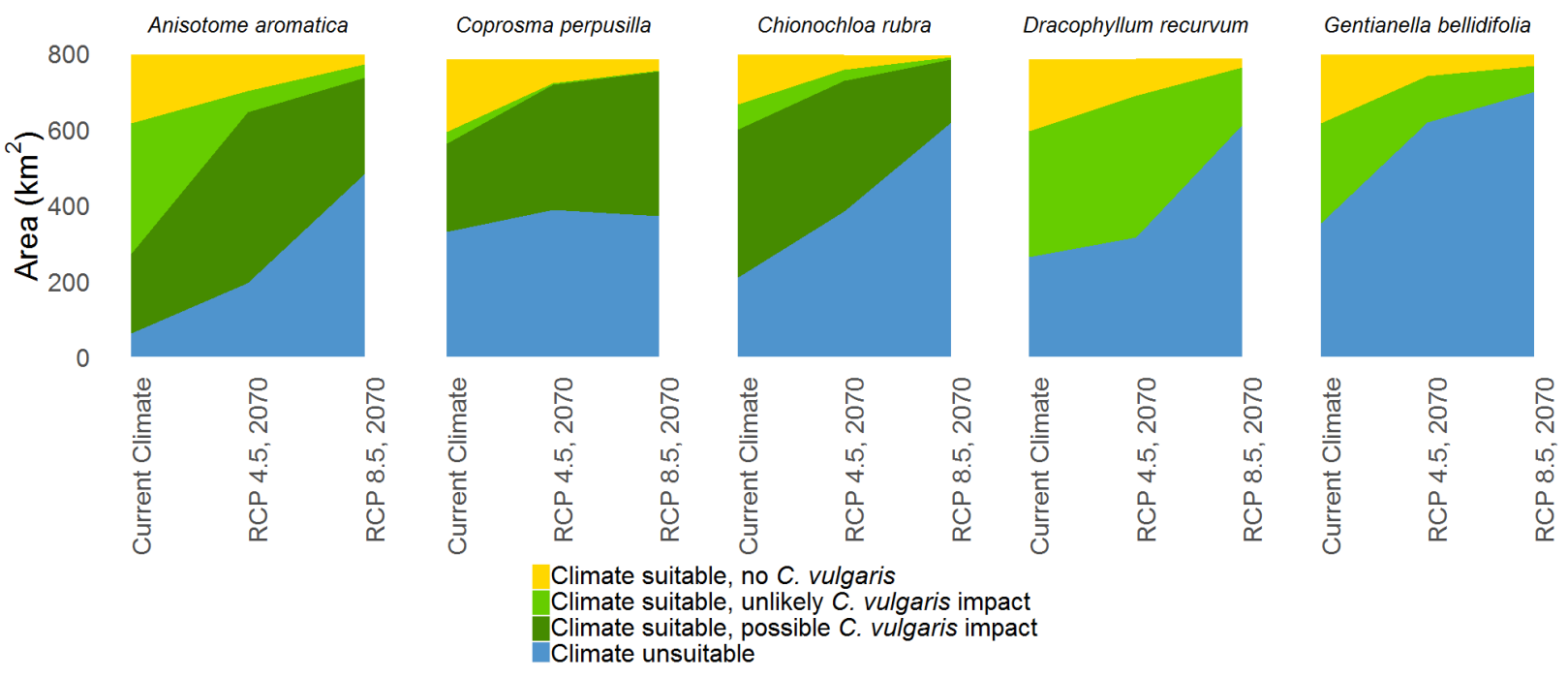

Supplementary Figure 28: Modelled suitable habitat area for five taonga tipu (sacred plants) in Tongariro National Park under current climate and two climate scenarios, RCP 4.5 (moderate emissions) and RCP 8.5 (severe emissions), for the year 2070.

The overall change in land area that is likely to be available to taonga tipu in TNP in the future represent substantial reductions in the amount of currently available habitat. Supplementary Table 11 contrasts the currently available habitat in TNP for taonga tipu with our predictions for the year 2070, under RCP 8.5, the "business as usual" $\mathrm{CO}_{2}$ emissions scenario. 
Supplementary Table 11: Area of Tongariro National Park ( $796 \mathrm{~km}^{2}$ total) that is predicted to be currently habitable by five alpine taonga tipu species and that will be habitable under climatic predictions for 2070 (IPCC RCP 8.5).

\begin{tabular}{|c|c|c|}
\hline Species & $\begin{array}{c}\text { Current available } \\
\text { habitat }\left(\mathrm{km}^{2}\right)\end{array}$ & $\begin{array}{l}\text { Available habitat in } \\
2070 \text { RCP8.5 }\left(\mathrm{km}^{2}\right)\end{array}$ \\
\hline Anisotome aromatica & 523.8 & 59.9 \\
\hline Chionochloa rubra & 195.9 & 11.0 \\
\hline Coprosma perpusilla & 225.9 & 33.1 \\
\hline Dracophyllum recurvum & 528.8 & 176.8 \\
\hline Gentianella bellidifolia & 443.3 & 96.3 \\
\hline
\end{tabular}




\section{Discussion}

In this study, we developed a novel modelling approach that allowed us to assess how both the direct impacts of climate change, as well as the changing geographic distribution and density of the invasive European heather (Calluna vulgaris (L.) Hull), are likely to affect the distributions and densities of five taonga tipu. To our knowledge, our study is the first to incorporate the density of an invasive species into predictions native species distributions in the future. As such, it significantly advances understanding of the combined effects of multiple drivers of global change on New Zealand's taonga.

We found that future climates will facilitate both an expansion of the geographic distribution and higher densities of $C$. vulgaris in TNP. For example, under RCP 8.5, which represents "business as usual" $\mathrm{CO}_{2}$ emissions in the year 2070 , only $3 \%\left(26 \mathrm{~km}^{2}\right)$ of TNP will remain climatically unsuitable for $C$. vulgaris. This represents an 8-fold decrease in the land area in TNP that currently acts as a $C$. vulgaris-free refuge for our native biodiversity, including taonga tipu. In contrast, future climates lead to dramatic declines in the availability of suitable habitats for our native alpine taonga tipu. For example, under RCP 8.5, we expect a drastic decrease in the land area that is climatically suitable for A. aromatica (from $524 \mathrm{~km}^{2}$ to only $60 \mathrm{~km}^{2}$ in 2070).

Perhaps most importantly, our research reveals a synergism between $C$. vulgaris invasion and climate warming is highly likely to lead to enhanced negative consequences for some alpine plants. We demonstrate that the most dramatic reductions in future suitable habitats occur for species that are sensitive to multiple agents of global change. For example, the indirect effects of increased competition of $C$. vulgaris on Chionochloa rubra under a warmer climate were large; incorporation of $C$. vulgaris effects in our models reduced the habitable areas for $C$. rubra by nearly two orders of magnitude (17.8-fold; from currently $195.9 \mathrm{~km}^{2}$ to only $11.0 \mathrm{~km}^{2}$ ). We suggest that $11 \mathrm{~km}^{2}$ of suitable habitat for the structurally and ecologically important tussock grass $C$. rubra is of serious concern for management of native biodiversity in TNP. Notably, our finding that species which are sensitive to multiple agents of global change are likely to experience the most dramatic reductions in suitable habitats is likely to be general in nature. This means that conservation managers throughout Aotearoa would be well-advised to 
prioritise management actions for plant species that are susceptible to multiple drivers of global change.

In addition to their cultural values, the taonga tipu modelled here represent a wide-range of taxa with unique growth forms, microhabitat preferences and ecologies within the alpine zone. Given the universally negative response of these species to the biotic and abiotic conditions that are likely to prevail in TNP in the future, our results suggest that the conservation of many native alpine plants is likely to become an even greater challenge in the future. Given the exceptional value TNP holds for New Zealand as a United Nations Educational, Scientific and Cultural Organization (UNESCO) Natural and Cultural World Heritage Site, as well as the importance of the New Zealand's alpine zones as hotspots of endemic biodiversity, these results suggest that conservation management of the park must urgently consider the impacts of multiple drivers of global change acting interactively on the resilience and integrity of our taonga. 


\section{Appendix 5: Supplementary materials to Chapter 5}

Climatic predictors selected $a$ priori to calibrate preliminary climatic envelope models

* Indicates inclusion in final calibrated model

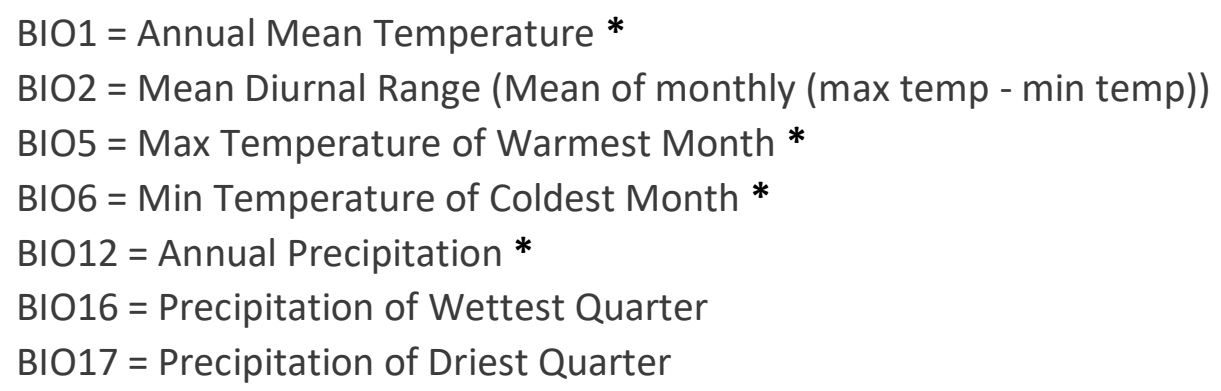

Vegetative cover classes deemed vulnerable to invasion by Calluna vulgaris in New Zealand

1. Improved pasture

2. Short tussock grassland

3. Broadleaved forest and scrub

4. Snow tussock grassland

5. Mixed indigenous scrub

6. Red tussock grassland

7. Urban

8. Pasture and broadleaved forest

9. Grassland and Leptospermum scrub or fern

10. Grassland and Dracophyllum scrub

11. Tussock grassland and subalpine scrub

12. Subalpine scrub

13. Podocarp-broadleaf beech forest and scrub

14. Grassland and gorse scrub

15. Horticultural crops and pasture

16. Beech forest and scrub

17. Beech-broadleaf forest and scrub

18. Exotic forest and scrub

19. Gorse Scrub

20. Grassland and Cassina scrub

21. Grassland and matagouri

22. Grassland and mixed indigenous scrub

23. Grassland and sweet briar and matagouri

24. Kauri and Leptospermum or mixed indigenous scrub

25. Leptospermum scrub or fern

26. Orchard or vineyards and pasture

27. Pasture and beech or Podocarp forest 
28. Pasture and exotic forest

29. Pasture and Podocarp-broadleaf forest

30. Podocarp-broadleaved-beech forest and scrub

31. Short tussock-snow tussock

32. Subalpine or alpine herbfield

33. Sub-alpine scrub and indigenous forest

34. Tussock grassland and beech forest

35. Tussock grassland and Podocarp-broadleaved forest

36. Unimproved pasture

Vegetative cover classes deemed unavailable to $C$. vulgaris in New Zealand

1. Beech forest

2. Broadleaved forest

3. Broadleaved forest and scrub

4. Exotic forest

5. Highland podocarp-broadleaved forest

6. Highland podocarp-broadleaved-beech forest

7. Ice

8. Lake

9. Lowland podocarp-broadleaved forest

10. Lowland podocarp-broadleaved-beech forest

11. None

12. Pakihi heartland communities

13. Podocarp forest

14. River

15. Sand-dune communities

16. Wetland communities

\section{Ericaceous species used to map possible mycorrhizal fungal presence}

1. Dracophyllum kirkii

2. Dracophyllum latifolium

3. Dracophyllum lessonianum

4. Dracophyllum longifolium

5. Dracophyllum menziesii

6. Dracophyllum pronum

7. Dracophyllum recurvum

8. Dracophyllum rosmarinifolium

9. Dracophyllum sinclairii

10. Dracophyllum strictum

11. Dracophyllum subulatum

12. Dracophyllum traversii

13. Dracophyllum uniflorum 
14. Dracophyllum urvilleanum

15. Dracophyllum viride

16. Epacris pauciflora

17. Erica lusitanica

18. Gaultheria depressa

19. Leucopogon fraseri

20. Pentachondra pumila 